\title{
Highly-Linearized CMOS Distributed Bidirectional Amplifier with Cross-Coupled Compensator for Wireless Communications
}

\author{
by \\ Ziad El-Khatib \\ A Ph.D. Thesis submitted to the \\ Faculty of Engineering \\ in Partial Fulfillment of the Requirements \\ for the Degree of \\ Doctor of Philosophy \\ program in \\ Ottawa-Carleton Institute for Electrical Engineering \\ Department of Electronics \\ Carleton University \\ Ottawa, Canada
}

(C) Ziad El-Khatib, 2012 
Library and Archives

Canada

Published Heritage

Branch

395 Wellington Street

Ottawa ON K1A ON4

Canada
Bibliothèque et

Archives Canada

Direction du

Patrimoine de l'édition

395 , rue Wellington

Ottawa ON K1A ON4

Canada
Your file Votre référence

ISBN: 978-0-494-89337-1

Our file Notre référence

ISBN: 978-0-494-89337-1

\section{NOTICE:}

The author has granted a nonexclusive license allowing Library and Archives Canada to reproduce, publish, archive, preserve, conserve, communicate to the public by telecommunication or on the Internet, loan, distrbute and sell theses worldwide, for commercial or noncommercial purposes, in microform, paper, electronic and/or any other formats.

The author retains copyright ownership and moral rights in this thesis. Neither the thesis nor substantial extracts from it may be printed or otherwise reproduced without the author's permission.
AVIS:

L'auteur a accordé une licence non exclusive permettant à la Bibliothèque et Archives Canada de reproduire, publier, archiver, sauvegarder, conserver, transmettre au public par télécommunication ou par l'Internet, prêter, distribuer et vendre des thèses partout dans le monde, à des fins commerciales ou autres, sur support microforme, papier, électronique et/ou autres formats.

L'auteur conserve la propriété du droit d'auteur et des droits moraux qui protege cette thèse. $\mathrm{Ni}$ la thèse ni des extraits substantiels de celle-ci ne doivent être imprimés ou autrement reproduits sans son autorisation.
In compliance with the Canadian Privacy Act some supporting forms may have been removed from this thesis.

While these forms may be included in the document page count, their removal does not represent any loss of content from the thesis.
Conformément à la loi canadienne sur la protection de la vie privée, quelques formulaires secondaires ont été enlevés de cette thèse.

Bien que ces formulaires aient inclus dans la pagination, il n'y aura aucun contenu manquant. 


\section{Abstract}

A highly-linear transmitter with fully-integrated broadband design linearization capability is required to address linearity improvements. When the input signal driven into the amplifier semiconductor is increased, the output is also increased until a point where distortion products can no longer be ignored. The harmonics and higher order distortion of the output signal are generated by nonlinearities of MOSFET devices. In response to the need to correct the broadband distributed amplifier (DA)'s nonlinear distortion, a number of DA linearization techniques have been developed. However, most of the published DA linearization methods reported do not provide fully-integrated distortion cancellation techniques with large third-order intermodulation (IM3) distortion reduction.

In this thesis, we demonstrate a fully-integrated fully-differential linearized CMOS distributed bidirectional amplifier that achieves a large IMD3 distortion reduction over broadband frequency range for both RF paths. The proposed linearized bidirectional DA has the drain and gate transmission-lines stagger-compensated. Reducing the DA IM3 distortion by mismatching the gate and drain LC delay-line ladders. The proposed fully-differential linearized DA employs a cross-coupled compensator transconductor to enhance the linearity of the DA gain cell with a nonlinear drain capacitance compensator for wider linearization bandwidth. The proposed linearized CMOS bidirectional DA achieves a measured IM3 distortion reduction of $20 \mathrm{~dB}$ with frequency of operation from $0.1 \mathrm{GHz}$ to $9.5 \mathrm{GHz}$ and a two-way amplification of $5 \mathrm{~dB}$ in both RF directions. The proposed linearized DA is implemented in $0.13 \mu \mathrm{m}$ RF CMOS process for use in highly-linear broadband communication. 


\section{Acknowledgements}

I would like to express my sincere gratitude to my thesis supervisors, Dr. Samy A. Mahmoud, Professor at the Faculty of Systems Engineering and Design at Carleton University and Dr. Leonard MacEachern Associate Professor at Electronics and Electrical Engineering at Carleton University for their continuous support, guidance, encouragement and involvement during the course of this work. Their help and support is greatly appreciated.

At Carleton University, I am in debt to all who provided support. Special thanks are also due to the fellow graduate students at the department of Electronics for their participation in interesting discussions. I would like to thank Zhan $\mathrm{Xu}$, Igor Miletic, AbdulHakim Ahmed, Kobe Situ, Arif Siddiqi, Greg Brzezina and to Mr. Nagui Mikhail for chip test set up help.

Financial support was provided by Carleton University in the form of a research assistantship and in the form of a graduate scholarship, and by the National Capital Institute of Telecommunications (NCIT) and Centre for Photonics Fabrication Research (CPFR) in the form of a research grant is gratefully acknowledged. I would like to thank the Canadian Microelectronics Corporation (CMC) staff for both Cadence design kits technical support and fabrication silicon space. I would like to thank IBM MOSIS labs for their chip fabrication support and Agilent staff for their ADS design environment tools and test equipment support to measure the chip performance.

I wish to thank my dear mother, my sisters and brothers for their continuous prayers, encouragement and support during the course of this thesis, they all have stood by me. 


\section{To my dear mother and family and friends for their love and kindness}




\section{Table of Contents}

$\begin{array}{ll}\text { Abstract } & \text { i }\end{array}$

Acknowledgements $\quad$ ii

Table of Contents $\quad$ iv

List of Tables vii

List of Figures viii

List of Symbols $\quad$ xvi

1 Introduction 1

1.1 Thesis Motivation . . . . . . . . . . . . . . . . . . . 1

1.2 Thesis Contributions $\ldots \ldots \ldots \ldots \ldots$

1.3 Outline of the Thesis $\ldots \ldots \ldots \ldots \ldots$

2 Modulation Schemes Effect on RF Power Amplifier Nonlinearity and RFPA Linearization Techniques $\quad 7$

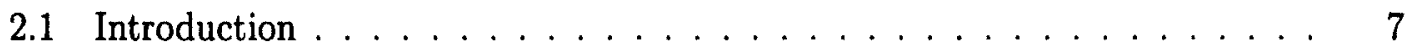

2.2 RF Modulation Scheme in Bandpass Radio Communication Channel . . . 7

2.2.1 Ideal Radio Transmitter . . . . . . . . . . . . . . . . . . . . . 9 9

2.2.2 RF Power Amplifier Linearity for Non-Modulated Signal . . . . . 10

2.2.3 RF Power Amplifier Linearity for Modulated Signals . . . . . . . . 17

2.2.4 RF Power Amplifier Spectral Regrowth - Out-of-band Distortion . . 19

2.3 Role of RF Power Amplifier Linearization Techniques . . . . . . . . . . . . 22

2.3.1 RF Power Amplifier Power Back-off . . . . . . . . . . . . . . . . 23

2.3.2 RF Power Amplifier Feedforward Linearization . . . . . . . . . . . . 24

2.3.3 RF Power Amplifier Cartesian Indirect Feedback Linearization . . . 26

2.3.4 RF Power Amplifier Polar Feedback Linearization . . . . . . . . . . 27

2.3.5 RF Power Amplifier RF Predistortion Linearization . . . . . . . . . 28 


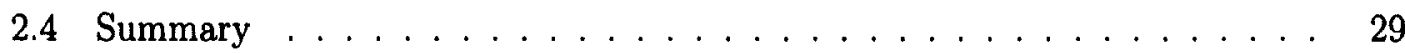

3 Distributed Amplification Principles and Transconductor Nonlinearity Compensation $\quad \mathbf{3 0}$

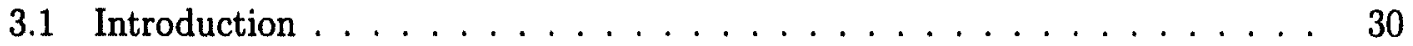

3.2 Distributed Amplification Principles . . . . . . . . . . . . . . . . 30

3.2.1 Additive Distributed versus Product Cascaded Amplification . . . . 31

3.2.2 Lumped Constant Delay Line Characteristics . . . . . . . . . . . . 35

3.2 .3 Lossless Distributed Amplification . . . . . . . . . . . . . . . . . . . 38

3.2.4 Lossy Distributed Amplification . . . . . . . . . . . . . . . . . . . 41

3.3 Transconductor Gain Cells for Fully-Differential Distributed Amplifiers . . 46

3.4 Chapter Summary . . . . . . . . . . . . . . . . . . . . . 51

4 Distributed RF Linearization Circuit Applications $\quad \mathbf{5 2}$

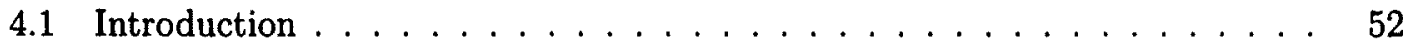

4.2 Linearized CMOS Distributed Active Power Splitter . . . . . . . . . . . . . 52

4.2.1 Amplitude and Phase Imbalance of Linearized CMOS Distributed

Active Power Splitter . . . . . . . . . . . . . . . . . . . . 55

4.2.2 CMOS Distributed Active Power Splitter Using Multiple-Gated Transistor Linearization . . . . . . . . . . . . . . . . . . 56

4.3 Linearized CMOS Distributed Matrix Amplifier Architecture . . . . . . . . 62

4.3.1 CMOS Distributed $2 \times 3$ Matrix Amplifier with Interleaved Distributed Loading Technique . . . . . . . . . . . . . . . . 63

4.3.2 Proposed CMOS Interleaved Distributed $2 \times 3$ Matrix Amplifier with Post Distortion and Gate Optimum Bias Linearization Technique . 66

4.4 Linearized CMOS Distributed Paraphase Amplifier . . . . . . . . . . . . 73

4.4.1 Amplitude and Phase Imbalance of Linearized CMOS Distributed Paraphase Amplifier . . . . . . . . . . . . . . . . 75

4.4.2 CMOS Distributed Paraphase Amplifier Employing Derivative Superposition Linearization . . . . . . . . . . . . . . . 77

4.5 Chapter Summary . . . . . . . . . . . . . . . . . . . . . . 80

5 Linearized CMOS Distributed Bidirectional Amplifier with Cross-Coupled $\begin{array}{lr}\text { Compensator } & \mathbf{8 2}\end{array}$

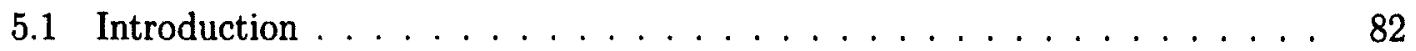

5.2 Linearized CMOS Distributed Bidirectional Amplifier Circuit Design Analysis 82

5.3 CMOS Cross-Coupled Compensator Transconductor as DA Gain Cell for Linearity Improvement and Enhanced Tunability . . . . . . . . . . . . 87

5.4 Effect of Nonlinear Drain Capacitance on DA Linearization Bandwidth . . 92

5.5 Transmission-Lines Multi-level Inductor Modeling in in Transmission-Lines for Silicon Chip Area Reduction . . . . . . . . . . . . . . . . . . . . 101 
5.5.1 Varactor-tuned LC Networks . . . . . . . . . . . . . . . . . 107

5.6 Chapter Summary . . . . . . . . . . . . . . . . . . . . . . 108

6 Linearized CMOS Distributed Bidirectional Amplifier Silicon Chip Im$\begin{array}{lr}\text { plementation } & 109\end{array}$

6.1 Introduction . . . . . . . . . . . . . . . . . . . . . . . . . . . . . 109

6.2 Linearized CMOS Bidirectional Distributed Amplifier High Frequency Layout Considerations . . . . . . . . . . . . . . . . . . . . . . . . 109

6.3 Silicon CMOS RF Multi-level Inductors Implementation . . . . . . . . . . 111

6.4 CMOS Bidirectional Distributed Amplifier Cross-coupled Compensator Gain Cell Layout . . . . . . . . . . . . . . . . . . . . . . . . . . . . 113

6.5 Linearized CMOS Bidirectional Distributed Amplifier Full Layout . . . . . 115

6.6 Chapter Summary . . . . . . . . . . . . . . . . . . . 118

7 Linearized CMOS Distributed Bidirectional Amplifier Experimental Setups and Chip Measurement Results 119

7.1 Introduction . . . . . . . . . . . . . . . . . . . . . 119

7.2 High-Frequency On-Wafer Measurement System . . . . . . . . . . . . . 119

7.3 S-parameter and Harmonics Power Measurements . . . . . . . . . . . . . 120

7.4 Noise Figure Setup and Measurement . . . . . . . . . . . . . . . 127

7.5 Summary . . . . . . . . . . . . . . . . . . . . 132

8 Summary of Thesis $\quad 135$

8.1 Summary . . . . . . . . . . . . . . . . . . . . 135

8.2 Future Work . . . . . . . . . . . . . . . . . . . . . 137

Appendix A List of Book, Journal and Conference Publications 138

A.1 List of Book, Journal and Conference Publications . . . . . . . . . . . . . 138

$\begin{array}{ll}\text { Appendices } & 138\end{array}$

$\begin{array}{lr}\text { Bibliography } & 140\end{array}$ 


\section{List of Tables}

1.1 Previous Related Published Work on Broadband Distributed Amplifier Linearization Techniques. . . . . . . . . . . . . . . . 4 4

2.1 One Tone Signal Generated Harmonics . . . . . . . . . . . . . . . . . . . 12

2.2 Two Tone Signal Generated Harmonics Coefficients . . . . . . . . . . . 15

2.3 Different PAR values for different modulated signal types ( $[1],[2])$. . . . 17

2.4 Comparison of Different RF Power Amplifier Linearization Techniques. . . 29

5.1 Simulated Performance of the linearized CMOS bidirectional distributed amplifier . . . . . . . . . . . . . . . . 108

7.1 Comparison with state-of-the-art previously published measured linearized distributed amplifiers highlighting the large $20 \mathrm{~dB}$ IMD3 reduction over broadband frequency range for both RF paths with least power consumption and minimum silicon chip area for the proposed bidirectional fullydifferential fully-integrated solution. . . . . . . . . . . . . . 129 


\section{List of Figures}

2.1 A General Universal Illustration of a Bandpass Communication Channel System. . . . . . . . . . . . . . . . . . . 8

2.2 Ideal radio quadrature upconverter transmitter. . . . . . . . . . . . 10

2.3 Output spectrum of a power amplifier that includes the desired fundamental signals as well as the spurious products created by intermodulation distortion. 13

2.4 Intermodulation products falling in-band. . . . . . . . . . . . . . 13

2.5 Output power versus input power defining third -order intercept point IP3, 15

2.6 RF Power Amplifier Spectral Regrowth. . . . . . . . . . . . . . . . . . 18

2.7 Effects of PA nonlinearity on adjacent channel power ratio. . . . . . . . . 21

2.8 Effects of PA nonlinearity on adjacent channel power ratio with third-order distortion. . . . . . . . . . . . . . . . . . . . . 22

2.9 Role of Linearization Techniques. . . . . . . . . . . . . . . . . . 23

2.10 RF Power Amplifier Power Back-off. . . . . . . . . . . . . . . . . . . 24

2.11 RF Power Amplifier Feedforward Linearization. . . . . . . . . . . . . 25

2.12 RF Power Amplifier Digitally Assisted Cartesian Feedback Linearization. . 26

2.13 RF Power Amplifier Polar Feedback Linearization. . . . . . . . . . . . . . . 27

2.14 RF Power Amplifier RF Predistortion Linearization. . . . . . . . . . . . . . 28

2.15 RF Power Amplifier Adaptive Digital Predistortion Linearization. . . . . . 28

3.1 A transmission-line constructed of inductors and capacitors coupled with amplification MOS devices. . . . . . . . . . . . . . . . . . . . . . 31

3.2 (a) Additive distributed amplification (b) Product cascaded amplification. $\quad 32$

3.3 (a) Constant-k T-sections (b) m-derived T-sections. . . . . . . . . . . . . 35

3.4 Lumped Constant Delay Line Characteristics . . . . . . . . . . . . . . . . . 35 
3.5 T-sections structure of gate and drain lines of the distributed amplifier. . . 42

3.6 Gate and drain transmission lines of a CMOS distributed amplifier. . . . . 42

3.7 (a) CMOS source degeneration transconductor (b) Caprio's cross-quad transconductor (c) Quinn's cascomp transconductor as a gain cell application for high-frequency amplifiers linearity enhancement. . . . . . . . . . . . 47

4.1 Block diagram illustration of CMOS distributed active power splitter. . . . 53

4.2 Simulated output power S21 common-source stage port and S31 commonsource stage port peaks at $7.8 \mathrm{~dB}$ for the proposed linearized CMOS distributed power splitter. . . . . . . . . . . . . . .

4.3 Simulated amplitude imbalance for the proposed linearized CMOS distributed power splitter amplitude imbalance. . . . . . . . . . . . . .

4.4 Simulated phase S21 and phase S31 for the proposed linearized CMOS distributed power splitter. . . . . . . . . . . . . . . . . 57

4.5 Simulated phase imbalance for the proposed linearized CMOS distributed

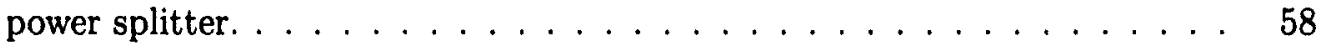

4.6 Schematic topology of the proposed fully-integrated linearized CMOS distributed active power splitter. . . . . . . . . . . . . . . . . . 59

4.7 ADS simulation of IIP3 before CMOS distributed active power splitter multiple-gated transistor linearization. . . . . . . . . . .

4.8 ADS simulation of IIP3 after CMOS distributed active power splitter multiplegated transistor linearization with an $8.5 \mathrm{~dB}$ IIP3 improvement and a $10 \mathrm{dBc}$ IMD3 improvement at output power of $-10 \mathrm{dBm} . \ldots \ldots \ldots$

4.9 Schematic illustration of multi-gated transistor linearization technique. The gate bias and the transistor size of the secondary transistor is chosen such that the negative $2^{\text {nd }}$ derivative of $g_{m}$ peak of main transistor is canceled by the positive one of secondary transistor. . . . . . . . . . .

4.10 Schematic topology of the proposed fully-integrated linearized CMOS interleaved distributed $2 \times 3$ matrix amplifier employing active post distortion and optimum gate bias linearization technique. . . . . . . . . . . . 
4.11 Simulated output power S21 peaks at 7.1 dB and S-parameters for the proposed linearized CMOS interleaved distributed $2 \times 3$ matrix amplifier. . . . 66

4.12 Simulated Two-Tone before linearization at $5 \mathrm{GHz} . \ldots \ldots 7$

4.13 Simulated Two-Tone after linearization at $5 \mathrm{GHz} . \ldots \ldots 8$

4.14 ADS Simulation of IIP3 before CMOS distributed $2 \times 3$ matrix amplifier active post distortion and optimum gate bias linearization. . . . . . . . 68

4.15 ADS Simulation of IIP3 after CMOS distributed $2 \times 3$ matrix amplifier active post distortion and optimum gate bias linearization with a $9 \mathrm{~dB}$ and a $18 \mathrm{dBc}$ improvement at output power of $-10 \mathrm{dBm} . \ldots . . . . . . . .669$

4.16 Simulated 1-dB gain compression point before linearization for the proposed linearized CMOS distributed $2 \times 3$ matrix amplifier. . . . . . . . . . . 70

4.17 Simulated 1-dB gain compression point after linearization for the proposed linearized CMOS interleaved distributed $2 \times 3$ matrix amplifier. . . . . . . 70

4.18 Simulated Two-Tone before active post distortion linearization only at 5 $\mathrm{GHz}$ for the proposed linearized CMOS interleaved distributed $2 \times 3$ matrix amplifier. . . . . . . . . . . . . . . .

4.19 Simulated Two-Tone after active post distortion linearization only at $5 \mathrm{GHz}$ for the proposed linearized CMOS interleaved distributed $2 \times 3$ matrix amplifier. . . . . . . . . . . . . . . . . . . . .

4.20 The VSWR for the proposed linearized CMOS interleaved distributed $2 \times 3$ matrix amplifier. . . . . . . . . . . . . . . . . 72

4.21 Block diagram illustration of CMOS distributed paraphase amplifier. . . . 73

4.22 Simulated Output Power S21 common-source stage port and S31 commongate stage port peaks at $5.3 \mathrm{~dB}$ for the proposed Linearized CMOS Distributed Paraphase Amplifier. . . . . . . . . . . . . . . . . .

4.23 Simulated amplitude imbalance for the proposed linearized CMOS distributed paraphase amplifier amplitude imbalance of less than $1.26 \mathrm{~dB} . \ldots$.

4.24 Simulated phase imbalance for the proposed linearized CMOS distributed paraphase amplifier with less than 10 degrees phase imbalance. . . . . . . .

4.25 Schematic Topology of the Proposed Fully-Integrated Linearized CMOS Distributed Paraphase Amplifier. . . . . . . . . . . . . . . . . . 78 
4.26 The derivative superposition linearization approach. The overall linearized transconductance is composed out of several gate bias shifted common source FET devices, which are current summed at their outputs. . . . . . . . .

4.27 ADS Simulation of IIP3 before CMOS distributed paraphase amplifier derivative superposition linearization. . . . . . . . . . . . .

4.28 ADS Simulation of IIP3 after CMOS distributed paraphase amplifier derivative superposition linearization with a $8.5 \mathrm{~dB}$ and a $13 \mathrm{dBc}$ improvement at output power of $-10 \mathrm{dBm} . \ldots \ldots \ldots$

5.1 A three-stage CMOS DA with MOSFET devices. . . . . . . . . . . . 83

5.2 (a) Designing a staggered bidirectional DA structure with mismatched timedelay for the drain and gate line will shift the IM3 attenuation frequency response inside the in-band of the DA cut-off frequency and in turn out the IM3 distortion (b) Further increase in the time-delay mismatch will shift the $I M_{3}$ attenuation frequency response inside the in-band of the DA cutoff frequency. . . . . . . . . . . . . . . .

5.3 More increase in time delay mismatch by increasing the drain and gate line capacitance mismatching will shift the IM3 attenuation frequency response even more inside the in-band of the distributed amplifier cut-off frequency further filtering out IM3 distortion. . . . . . . . . . . . . .

5.4 (a) Proposed topology of the three-stage bidirectional distributed amplifier with CMOS cross-coupled compensator transconductor gain cells coupling the staggered drain and gate transmission-lines with nonlinear drain capacitance compensator (b) A varactor-based active post nonlinear drain capacitance compensator in the CMOS distributed structure for wider linearization bandwidth. . . . . . . . . . . . . . . . . . . . 99 90

5.5 CMOS cross-coupled compensator tunable gm flatness versus input signal. 91

5.6 Simulation result of CMOS cross-coupled compensator wide tunable gm" nulling versus input signal. . . . . . . . . . . . . . . . . . 93 
5.7 The proposed schematic of the three-stage bidirectional distributed amplifier with CMOS cross-coupled compensator transconductor gain cells coupling the staggered drain and gate transmission-lines with nonlinear drain capacitance compensator. . . . . . . . . . . . . . . . . . . . . .

5.8 Simulated differential $S_{21}$ power gain, $S_{11}$ and $S_{22}$ return losses and $S_{12}$ isolation for the three-stage fully-differential linearized CMOS bidirectional

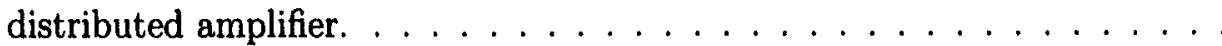

5.9 Simulated IIP3 before and after linearization for linearized CMOS bidirectional distributed amplifier. . . . . . . . . . . . . . . . . . 97

5.10 Simulated IM3 before linearization at $1 \mathrm{GHz}$ for linearized CMOS bidirectional distributed amplifier. . . . . . . . . . . . . . . . 98

5.11 Simulated IM3 after linearization at $1 \mathrm{GHz}$ for linearized CMOS bidirectional distributed amplifier. . . . . . . . . . . . . . . . . . 98

5.12 Simulated IM3 before linearization at $5 \mathrm{GHz}$ for linearized CMOS bidirectional distributed amplifier.

5.13 Simulated IM3 after linearization at $5 \mathrm{GHz}$ for linearized CMOS bidirectional distributed amplifier.

5.14 Simulated fundamental and third-order intermodulation distortion for onestage CMOS cross-coupled cascomp transconductor. . . . . . . . . . . . . . 100

5.15 Simulated stability factor $K_{f}$ and $B_{1 f}$ characterizing linearized bidirectional distributed amplifier stability. . . . . . . . . . . . . . . . . . . . . 101

5.16 HFSS linearized CMOS bidirectional distributed amplifier transmission-lines multi-level inductor modeling RF CMOS $0.13 \mu \mathrm{m}$. The HFSS modeled inductor has an outer diameter of $91 \mu \mathrm{m}$ and spacing of $5 \mu \mathrm{m}$ with width of

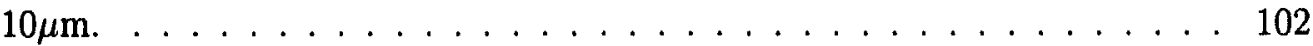

5.17 ADS linearized CMOS bidirectional distributed amplifier transmission-lines multi-level inductor modeling RF CMOS $0.13 \mu \mathrm{m}$. 
5.18 HFSS and ADS linearized CMOS bidirectional distributed amplifier gate transmission-lines multi-level inductor modeling $\mathrm{Q}$ and L Modeling RF CMOS $0.13 \mu \mathrm{m}$. The ADS EM calculations were performed using the methods of moments where as in HFSS the 3D full-wave EM calculations were performed using the finite element method. . . . . . . . . . . . . . . . . 103

5.19 ADS transmission-lines multi-level inductor broadband equivalent circuit model in RF CMOS $0.13 \mu \mathrm{m}$. The multi-level inductor broadband equivalent circuit component values were determined by curve-fitting with the EM HFSS and ADS simulated results. . . . . . . . . . . . . . 105

5.20 HFSS and ADS drain transmission-line multi-level inductor $Q$ and $L$ modeling for RF CMOS $0.13 \mu \mathrm{m} \ldots \ldots \ldots$

5.21 (a) MOSCAP varactor test setup schematic with source and drain connected together to generate a highly non-linear capacitance-voltage curve behavior (b) C-V characteristic of the minimum and maximum MOSCAP varactor capacitance. . . . . . . . . . . . . . . . . . . . . . 107

6.1 Micrograph of Silicon RF CMOS $0.13 \mu \mathrm{m}$ multi-level drain line inductors with outer diameter of $91 \mu \mathrm{m}$ and spacing of $5 \mu \mathrm{m}$ and width of $10 \mu \mathrm{m}$ and number of turns $\mathrm{n}$ of $1.75 \ldots \ldots \ldots 111$

6.2 Cadence layout of silicon RF CMOS $0.13 \mu \mathrm{m}$ multi-level inductor. . . . . . 113

6.3 Silicon RF CMOS $0.13 \mu \mathrm{m}$ linearized bidirectional distributed amplifier full active cross-coupled compensator gain cell with enhanced tunability. . . . . 114

6.4 Silicon RF CMOS $0.13 \mu \mathrm{m}$ linearized bidirectional distributed amplifier crosscoupled compensator gain cell with multi-level inductors. . . . . . . . . 115

6.5 Silicon RF CMOS $0.13 \mu \mathrm{m}$ linearized bidirectional distributed amplifier codesigned with on-chip antenna. The on-chip differential loop antenna with dimension $280 \mu \mathrm{m} \times 220 \mu \mathrm{m}$ designed in HFSS. . . . . . . . . . . . . . . 116

6.6 Fully-integrated silicon RF CMOS $0.13 \mu \mathrm{m}$ linearized bidirectional distributed amplifier layout co-designed with on-chip antenna with multi-level inductors. The total chip silicon area is $1.5 \mathrm{~mm}^{2}$ including testing pads. . . . 117 
6.7 Micrograph of fully-integrated fully-differential silicon RF CMOS $0.13 \mu \mathrm{m}$ linearized bidirectional distributed amplifier co-designed with on-chip antenna. 117

7.1 On-wafer RF automated GPIB chip testing of the $0.13 \mu \mathrm{m}$ RF CMOS linearized CMOS distributed bidirectional amplifier. . . . . . . . . . . 120

7.2 RF automated GPIB testing of the $0.13 \mu \mathrm{m}$ RF CMOS three-stage fullydifferential linearized CMOS distributed bidirectional amplifier with highfrequency $\mathrm{RF}$ probes. . . . . . . . . . . . . . . . . . . . 121

7.3 Test setup for S-parameter measurements for the linearized CMOS distributed bidirectional amplifier. . . . . . . . . . . . . . . . . . . 122

7.4 (a) Two-tone test setup for measuring power and intermodulation distortion

(b) Vector digitally modulated signals generator measurement setup. . . . 123

7.5 IIP3 Comparison of measured reverse path output power versus input power with and without linearization for both (a) forward and (b) reverse RF paths.124

7.6 Comparison of simulation and measured differential S-parameters for both (a) forward and (b) reverse RF paths. . . . . . . . . . . . . . . . . 124

7.7 Comparison of measured output spectra with and without linearization for two-tones frequencies at $1 \mathrm{GHz}$ with $100 \mathrm{MHz}$ spacing for both (a) forward and (b) reverse $\mathrm{RF}$ paths. . . . . . . . . . . . . . . . . . 125

7.8 Comparison of measured output spectra with and without linearization for two-tones frequencies at $3 \mathrm{GHz}$ with $100 \mathrm{MHz}$ spacing for both (a) forward and (b) reverse $\mathrm{RF}$ paths.

7.9 Comparison of measured reverse path output spectra with and without linearization for two-tones frequencies at $5 \mathrm{GHz}$ with $100 \mathrm{MHz}$ spacing for both (a) forward and (b) reverse RF paths. . . . . . . . . . . . . . . . . 127

7.10 Comparison of measured output spectra with and without linearization for two-tones frequencies at $5.9 \mathrm{GHz}$ with $100 \mathrm{MHz}$ spacing for both (a) forward and (b) reverse $\mathrm{RF}$ paths. . . . . . . . . . . . . . . . . 128

7.11 Comparison of the measured IM3 distortion reduction with and without linearization over broadband frequency of operation for both (a) forward and (b) reverse $\mathrm{RF}$ paths. . . . . . . . . . . . . . . 128 
7.12 Measured output PSD with and without linearization at $2 \mathrm{GHz}$ for $\Pi / 4$ DQPSK digitally modulated carrier signal using a square root Nyquist filter using a roll-off factor $\beta=0.35$. Spectral regrowth due to amplifier nonlinearities are fully compensated for, resulting in nearly $20 \mathrm{~dB}$ improvement in ACI relative to the output signal. . . . . . . . . . . . . . . . . . . . . 130

7.13 Measured output PSD with and without linearization at $2 \mathrm{GHz}$ for 10-Tones $5 \mathrm{MHz}$ bandwidth each Multi-tone modulated signal. . . . . . . . . . . . 131

7.14 Measured output PSD with and without linearization at $2 \mathrm{GHz}$ for 4-QAM digitally modulated carrier signal. . . . . . . . . . . . . . . . . . . . . 131

7.15 Comparison of the IM3 distortion reduction under different varactor bias voltage adjustment from $1 \mathrm{~V}$ to $1.8 \mathrm{~V}$ there exist an optimal bias at which IM3 suppression is formed at $1.45 \mathrm{~V} \ldots \ldots \ldots$. . . . . . . . . . . . . . 132

7.16 Noise figure calibration and measurement setup for the linearized CMOS distributed bidirectional amplifier. . . . . . . . . . . . . . . . . . 133

7.17 Measured noise figure for the linearized CMOS distributed bidirectional amplifier.

7.18 Measurement setup of of the $0.13 \mu \mathrm{m}$ RF CMOS three-stage fully-differential linearized CMOS distributed bidirectional amplifier on analytical probe station. 


\section{List of Symbols}

ACPR Adjacent Channel Power Ratio

$A_{d} \quad$ Drain line attenuation per section

$A_{g} \quad$ Gate line attenuation per section

$A_{v} \quad$ Voltage gain

ADS Advanced Design System

ASITIC Analysis and Simulation of Spiral Inductors and Transformers for IC

CDMA Code Division Multiple Access

$C_{g s} \quad$ Gate-source capacitance

$C_{g d} \quad$ Gate-drain capacitance

$C_{o x} \quad$ Capacitance of oxide

$C_{s i} \quad$ Capacitance of silicon substrate

CMOS Complementary Metal Oxide Semiconductor

$d B_{c} \quad$ decibel relative to a carrier level

DA Distributed Amplifier

dB Decibel

$\mathrm{dBm}$ Decibel referenced to $1 \mathrm{~mW}$ 
DQPSK Differential Quadrature Phase Shift Modulation

EM Electromagnetic

EVM Error Vector Magnitude

ESD Electrostatic Discharge

$\varepsilon_{r} \quad$ Relative dielectric constant of substrate

$f_{\max }$ Maximum frequency of oscillation

f Frequency in Hertz

$f_{\text {res }}$ Resonance Frequency

$f_{T} \quad$ Unity current-gain frequency

GPIB General Purpose Interface Bus

$g_{m} \quad$ Transconductance

GBW Gain-bandwidth product

HFSS High Frequency Structure Simulator

IM3 Third-order (in-band) intermodulation distortion

IIP3 Input Third-order intercept point

IBO Input Back-off

IM Intermodulation

IMD intermodulation distortion

$I_{d} \quad$ Drain current

IM/DD Intensity Modulation Direct Detection

IF Intermediate Frequency

xvii 
$L_{d} \quad$ Output drain line inductance

$L_{g} \quad$ Input gate line inductance

Mbps Mega Bits Per Second

MGTR Multi-Gate Transistor

MIM Metal-Insulator-Metal

NADC North American Digital Cellular

N Number of DA Gain Stages

$N_{\text {opt }}$ Optimum number of gain stages

$N F \quad$ Noise Figure

OFDM Orthogonal Frequency Division Multiplexing

OIP3 Output Third-order intercept point

PAR Peak to Average Power Ratio

PSD Power Spectral Density

$P_{1 d B} \quad 1 \mathrm{~dB}$ Compression Point

$P_{\text {out }}$ Output power

QAM Quadrature Amplitude Modulation

Q Quality factor

RFPA RF Power Amplifier

RF Radio Frequency

$R_{s i} \quad$ Resistance of silicon substrate

RF Radio Frequency 
RoF Radio-over-Fiber

SRF Self-resonant frequency

THD Total harmonic distortion

UWB Ultra wideband

$\omega_{c} \quad$ Cut-off frequency

$Z_{I} \quad$ Image impedance

$Z_{0} \quad$ Transmission line characteristic impedance 


\section{Chapter 1}

\section{Introduction}

\subsection{Thesis Motivation}

Transmitter nonlinearity produces harmonics and intermodulation distortion (IMD) products. Some of these products fall within the transmission band and can degrade system performance. For good service quality, limitations set on nonlinearity in the transmitter. It is required to keep the distortion below a certain level ( [3], [4]). Cable TV networks requires a carrier to distortion ratio better than $50 \mathrm{~dB}$ ( [4], [5]). Other wireless services such as micro-cellular and pico-cellular require a dynamic range up to $90 \mathrm{~dB}$ and $55 \mathrm{~dB}$ respectively ( [5], [6]). Thus making a highly-linear transmitter necessary in order to achieve the required signal dynamic range [7]. Linearization techniques are often employed to the power amplifier transmitter to improve system performance.

Increasing demand for spectral efficiency in radio communications makes multi-level linear modulation schemes, such as quadrature amplitude modulation (QAM) and orthogonal frequency division multiplexing (OFDM), in more demand ( $[8],[9])$. However, the use of such linear modulation schemes have increased the linearity requirements of RF components such as power amplifiers. Since their signals' envelopes fluctuate, these modulation schemes are more sensitive to power amplifier nonlinearities, the major contributor of nonlinear distortion in a transmitter. Therefore the power amplifier is required to process high data rate non-constant envelope signals ( [10], [11]). For achieving good power efficiency the power amplifier should work around its compression point which makes the output signal distorted nonlinearly. These nonlinear distortions generate in-band interferences which 
results in amplitude and phase deviation of the modulated vector signal. It also generates out-band interference in the adjacent channel creating spectrum spreading. When a non-constant envelope signal goes through a nonlinear PA, spectral regrowth broadening appears in the PA output causing adjacent channel interference [10]. PA linearization is often necessary to suppress spectral regrowth and reduce bit error rate (BER).

MOSFET devices can be modeled by nonlinear current sources and nonlinear capacitance that depend on the device voltages ( [12], [13]). These nonlinear sources will give rise to distortion when driven with a modulated signal. The real MOSFET device output impedance is nonlinear and the mobility $\mu$ is not a constant but a function of the vertical and horizontal electric field. There is also an internal feedback so when the input signal driven into the amplifier is increased, the output is also increased until a point where distortion products can no longer be ignored ( [12], [14]).

The harmonics of the output signal are generated by nonlinearities of the MOSFET devices. The major three nonlinear elements of the MOSFET devices are nonlinear transconductance $g_{m}$, the device drain capacitance $C_{d}$ and gate capacitance $C_{g s}([12],[15])$. The real MOSFET devices generate higher order distortion ( $[16],[12])$. Models are used to characterize the nonlinear behavior of a semiconductor device in order to predict the resultant signal properties. The simple polynomial approximation is a nonlinear transfer function based upon the Taylor series expansion ( [16], [15]).

Ideal RF power amplifier has an output signal that is a precise scaled copy of the input signal. Unfortunately real amplifiers are characterized by some degree of nonlinearity. No transistor is perfectly linear since the inherent nonlinearity of the diode junctions that comprise many of the active devices found in most amplifiers. Challenges in broadband amplifiers is to maintain high linearity over the entire bandwidth. Narrow band linearization techniques can not be utilized. Fully-integrated broadband linearization techniques are required to address linearity improvements for broadband communication systems ( [17], [18]). Traditional broadband amplifiers only allow broadband amplification in one direction. However integrating the bidirectional element into a linearized DA allows for broadband amplification in both directions eliminating the need for RF switches that degrade performance and increase insertion loss. The versatility of broadband distributed amplifier (DA) configurations makes them useful for performing several circuit functions 
integrated in wireless transceivers ( [19], [20], [21], [22]). High-Linearity RF Broadband Amplifiers are used in many wireless applications such as base station transceivers, CATV distribution systems, laser diode driver and UWB Communications.

In response to the need to correct the DA's nonlinear distortion, a number of DA linearization techniques have been developed and have been reported in literature ( [7] - [23]). However, most of the published DA linearization methods reported do not provide fully-integrated distortion cancellation technique with large third-order intermodulation (IM3) distortion reduction. Since they involve system-level linearization with bulky discrete components which is not suited for fully-integrated circuit miniaturization. Due to the discrete component performance variation with frequency, they suffer from limited linearization over broad bandwidth ( [7], [23]). Other DA linearization techniques involve circuit-level linearization, however they have narrow linearized bandwidth and apply DCbased linearization technique only ( [24], [25]). Previous methods do not provide large IM3 distortion cancellation and large spectral regrowth reduction as shown in Table 1.1.

A CMOS DA based multi-tanh linearization technique is also reported in literature [26]. However the CMOS DA based multi-tanh linearization technique offered a limited 5 dB IM3 distortion reduction only [26]. Another linearized DA that has been reported in literature is a differential DA with circuit-level feedforward linearization technique [27]. It operated over a very wide band from $0.1-12 \mathrm{GHz}$, however only simulation results were presented [27]. Lau and Chan proposed a linearized DA that achieved a $10 \mathrm{~dB}$ IM3 reduction over a limited $2.3 \mathrm{GHz}$ bandwidth range [23] as shown in Table 1.1. Recently, Lu and Pham ( [28], [29]) proposed a multi-gated transistor (MGTR) topology based CMOS linearized DA. The MGTR-based linearized distributed amplifier operated over a limited bandwidth of $4 \mathrm{GHz}$ range and had only a $11 \mathrm{~dB}$ IM3 reduction. Comparing the proposed CMOS linearized DA in this work [30] to other published ones, the proposed linearized CMOS DA offers a large IM3 distortion cancellation and large spectral regrowth reduction of $20 \mathrm{~dB}$ with $9.5 \mathrm{GHz}$ operational bandwidth and with the least power consumption [30]. The proposed approach offers full integration in standard CMOS technology for broadband applications.

In this thesis, we demonstrate a fully-integrated fully-differential linearized CMOS distributed bidirectional amplifier that achieves large IMD3 distortion reduction over broadband frequency range for both RF paths. The drain and gate transmission-lines were 
Table 1.1: Previous Related Published Work on Broadband Distributed Amplifier Linearization Techniques.

\begin{tabular}{|l||c|c|c|c|c|c|c|c|}
\hline $\begin{array}{l}\text { Design } \\
\text { Ref. }\end{array}$ & $\begin{array}{l}\text { Technology } \\
\text { Process }\end{array}$ & $\begin{array}{l}\text { Power } \\
\text { Gain } \\
(\mathrm{dB})\end{array}$ & $\begin{array}{l}\text { Operational } \\
\text { Bandwidth } \\
(\mathrm{GHz})\end{array}$ & $\begin{array}{l}\text { IMD3 } \\
\text { Reduction } \\
(\mathrm{dB})\end{array}$ & $\begin{array}{l}\text { IIP3 } \\
(\mathrm{dBm})\end{array}$ & $\begin{array}{l}\text { Power } \\
\text { Consump. } \\
(\mathrm{mW})\end{array}$ & $\begin{array}{l}\text { Linearization } \\
\text { Technique }\end{array}$ & $\begin{array}{l}\text { Circuit } \\
\text { Topology } \\
\text { Chip Area } \mathrm{mm}^{2}\end{array}$ \\
\hline$[17]$ & $\begin{array}{c}\text { GaAs } \\
\text { HEMT }\end{array}$ & NA & 10 & 15 & NA & NA & Feedforward & $\begin{array}{c}\text { Discrete } \\
\text { Components }\end{array}$ \\
\hline$[7]$ & $\begin{array}{l}\text { SiGe } \\
\text { BJT }\end{array}$ & 14 & 2.2 & 12 & NA & NA & $\begin{array}{c}\text { Parallel } \\
\text { Diode }\end{array}$ & $\begin{array}{c}\text { Discrete } \\
\text { Components }\end{array}$ \\
\hline$[23]$ & $\begin{array}{c}\text { SiGe } \\
\text { BJT }\end{array}$ & 13 & 2.3 & 10 & 10 & 180 & $\begin{array}{c}\text { Self } \\
\text { Biased }\end{array}$ & $\begin{array}{c}\text { Discrete } \\
\text { Components }\end{array}$ \\
\hline$[24]$ & $\begin{array}{c}\text { GaAs } \\
\text { MESFET }\end{array}$ & NA & 2.5 & 20 & NA & NA & $\begin{array}{c}\text { Derivative } \\
\text { Superposition }\end{array}$ & $\begin{array}{c}\text { Fully Integrated } \\
\text { NA }\end{array}$ \\
\hline $\begin{array}{l}\text { InGaP } \\
\text { GaAs HBT }\end{array}$ & 7.5 & 11 & 7 & 22 & NA & $\begin{array}{c}\text { Optimum Bias } \\
\text { Condition }\end{array}$ & $\begin{array}{c}\text { Fully Integrated } \\
\text { NA }\end{array}$ \\
\hline$[31]$ & $\begin{array}{c}\text { CMOS } \\
0.18 \mu \mathrm{m}\end{array}$ & 7 & 7.5 & 5 & 19 & 158 & Multi-Tanh & $\begin{array}{c}\text { Fully Integrated } \\
2.4 m^{2}\end{array}$ \\
\hline
\end{tabular}


stagger-compensated. Reducing the DA IM3 distortion by mismatching the gate and drain LC delay-line ladders. A CMOS cross-coupled compensator transconductor is proposed, in section 5.3, to enhance the linearity of the DA gain cell with a varactor-based active post nonlinear drain capacitance compensator for wider linearization bandwidth. The proposed linearized CMOS bidirectional DA achieves a measured IM3 distortion reduction of 20 $\mathrm{dB}$ over ultra-wideband from $0.1 \mathrm{GHz}$ to $9.5 \mathrm{GHz}$ frequency of operation with a two-way amplification of $5 \mathrm{~dB}$ in both RF directions. It is implemented in $0.13 \mu \mathrm{m}$ RF CMOS technology with a silicon chip area of $1.5 \mathrm{~mm}^{2}$ for use in highly-linear wireless communication systems.

\subsection{Thesis Contributions}

The main contribution of this thesis research is the realization a fully-integrated highfrequency active broadband linearizer for large IM3 distortion cancellation and spectral regrowth reduction in standard CMOS technology. The specific thesis contributions are as follows:

1. The development of linearized CMOS stagger-compensated bidirectional distributed amplifier with $20 \mathrm{~dB}$ IM3 distortion reduction.

2. The development of a highly-linear CMOS cross-coupled compensator transconductor with enhanced tunability.

3. The application of linearization techniques to distributed circuit designs such as power splitters, matrix amplifier and paraphase amplifier.

\subsection{Outline of the Thesis}

The thesis objectives are introduced in Chapter 1. In Chapter 2, modulation schemes effects on RF power amplifier nonlinearity and RFPA linearization techniques are presented. In Chapter 3, distributed amplification principles and transconductor nonlinearity compensation are presented. Various applications of linearized distributed circuit functions are presented in Chapter 4. Chapter 5 describes in detail the proposed fully-integrated linearized CMOS bidirectional distributed amplifier and the proposed highly-linear CMOS 
cross-coupled compensator transconductor with enhanced tunability. Chapter 6 presents the proposed linearized CMOS bidirectional distributed amplifier layout techniques and considerations. Chapter 7 presents the proposed linearized CMOS bidirectional DA experimental test setups and measured results. Chapter 8 draws conclusions of the thesis work and lists thesis contributions. 


\section{Chapter 2}

\section{Modulation Schemes Effect on RF Power Amplifier Nonlinearity and RFPA Linearization Techniques}

\subsection{Introduction}

In this chapter, RF modulation schemes effects on RF power amplifier nonlinearities are presented. A review of various power amplifier RF linearization techniques are discussed.

\subsection{RF Modulation Scheme in Bandpass Radio Com- munication Channel}

In radio communications, modulation can be described as the process of conveying a message signal by superimposing an information bearing signal onto a carrier signal by varying the signal characteristic ( [32], [33], [34]). Modulation is the process of changing a higher frequency signal in proportion to a lower frequency one or vise versa. The higher frequency signal is referred to as the carrier signal and the lower frequency signal is referred to as the information bearing message signal or modulating signal ( [32], [33], [34]). The characteristics (amplitude, frequency or phase) of the carrier signal are varied in accordance with the information bearing signal. These high-frequency carrier signals can be transmitted over the air, over fiber or coax cable. The use of high frequency signals will make the amplifier and antenna design easier for effective radio design ( [33], [34]).

Figure 2.1 shows the up conversion of the complex-valued baseband signal $x^{\prime}(t)$ to 


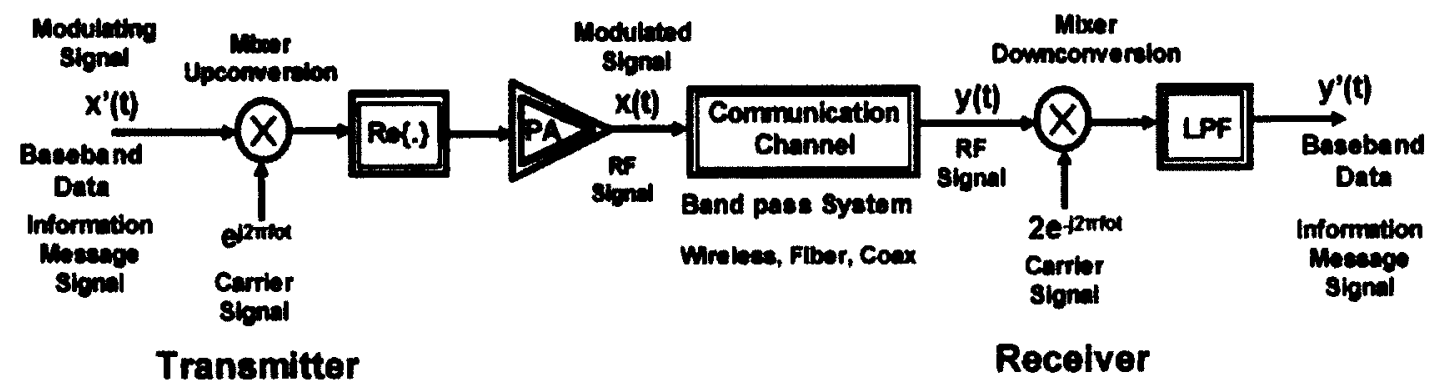

Figure 2.1: A General Universal Illustration of a Bandpass Communication Channel System.

the passband then the transmission of the real-valued bandpass signal $x(t)$ through the communication channel [32]. After the bandpass signal goes through the channel, a downconversion of the bandpass output $Y(t)$ into a complex-valued baseband signal $Y^{\prime}(t)$ occurs. The baseband signal $x^{\prime}(t)$ is up-converted to the bandpass signal by amplitude, phase or frequency modulation in order to transmit it. The modulated bandpass signal $x(t)$ can be described as

$$
x(t)=A(t) \cos \left(2 \pi f_{o} t+\phi(t)\right)
$$

where $f_{o}$ is the carrier frequency, $\mathrm{A}(\mathrm{t})$ is the amplitude and $\phi(\mathrm{t})$ is the phase modulation.

The bandpass signal $x(t)$ has an envelope bandwidth lower then the carrier frequency $f_{0}$. Using trigonometric identities, this signal can be re-written as ( [35], [34], [33])

$$
x(t)=I(t) \cos \left(2 \pi f_{c} t\right)-Q(t) \sin \left(2 \pi f_{c} t\right)
$$

where $I(t)$ is the in-phase component and $Q(t)$ is the quadrature component

$$
\begin{aligned}
& I(t)=A(t) \cos [\theta(t)] \\
& Q(t)=A(t) \sin [\theta(t)]
\end{aligned}
$$

Consequently, the bandpass signal $x(t)$ can be re-written in complex form as

$$
x(t)=A(t) \cos [\theta(t)] \cos \left(2 \pi f_{c} t\right)-A(t) \sin [\theta(t)] \sin \left(2 \pi f_{c} t\right)
$$




$$
\begin{gathered}
x(t)=A(t) \cos \left[2 \pi f_{c} t+\theta(t)\right]=\operatorname{Re}\left[A(t) e^{j \theta(t)} e^{j 2 \pi f_{c} t}\right] \\
x(t)=\operatorname{Re}\left[x^{\prime}(t) e^{j 2 \pi f_{c} t}\right]
\end{gathered}
$$

where $x^{\prime}(t)$ is the baseband input signal and can be represented as

$$
\begin{gathered}
x^{\prime}(t)=I(t)+j Q(t) \\
x^{\prime}(t)=A(t) e^{j \theta(t)}
\end{gathered}
$$

Therefore, the real-valued bandpass input signal $x(t)$ can be obtained from the complex valued baseband input signal $x^{\prime}(t)$ as ( [32], [34])

$$
x(t)=\operatorname{Re}\left\{x^{\prime}(t) e^{j 2 \pi f_{0} t}\right\}
$$

where $f_{o}$ is the carrier frequency. Similarly, the baseband output signal $\mathrm{y}^{\prime}(\mathrm{t})$ can be obtained from the bandpass output signal $\mathrm{y}(\mathrm{t})$ through demodulation process $2 e^{-j 2 \pi f_{o} t}$.

The communication channel can be linear or nonlinear. The choice of a modulation scheme depends on the physical characteristics of this channel, required levels of performance and hardware trade-offs [34].

\subsubsection{Ideal Radio Transmitter}

As shown in Figure 2.2 the baseband processor converts the information we want to send into data. The data can be either analog (continuously varying) or digital (in discrete states) in format ( [32], [34], [36]).

An ideal radio quadrature upconverter transmitter generates a high-frequency carrier for the data information to ride on. To do this we use a component called an oscillator as shown in Figure 2.2. Ideal radio quadrature upconverter transmitter as shown in Figure 2.2 has an oscillator that converts DC bias into a radio-frequency carrier. Then the carrier is combined with the data using a component called a modulator ( [34], [33], [36]). The data adjusts or modulates the characteristics of the carrier (amplitude, frequency or phase) 


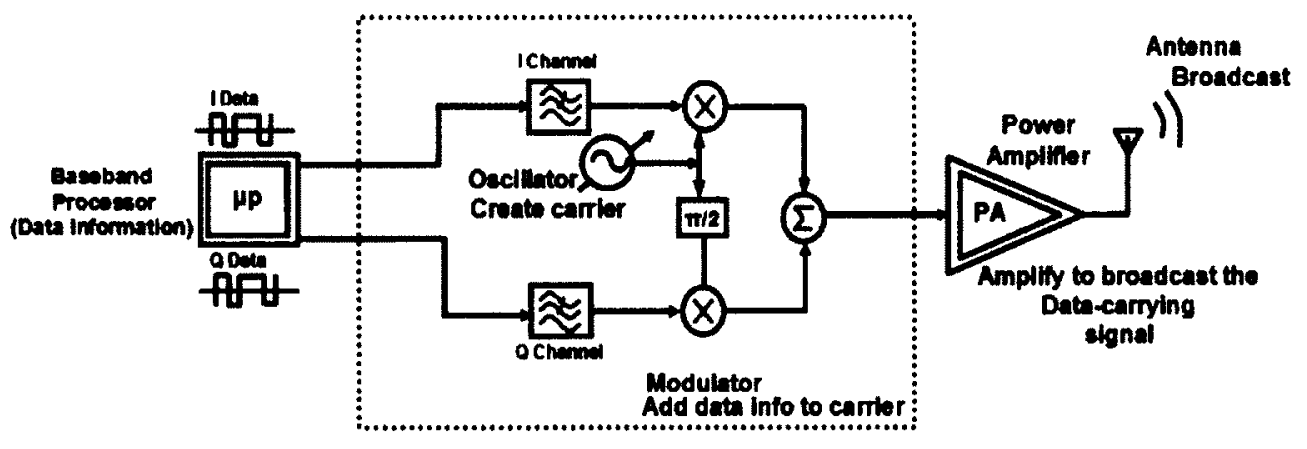

Figure 2.2: Ideal radio quadrature upconverter transmitter.

in a controlled manner. The third step is to increase the signal strength with a power amplifier so that it can be detected by the receiver. The output of the power amplifier feeds an antenna which broadcasts the information carrying signal into the air. It also can be transmitted through other communication mediums such as fiber or coax cable $([34],[33],[36])$.

\subsubsection{RF Power Amplifier Linearity for Non-Modulated Signal}

MOSFET devices can be modeled by nonlinear current and nonlinear capacitance that depend on the device voltages ( [12], [13]). These nonlinear sources will give rise to distortion when driven with a modulated signal. The real MOSFET device output impedance is nonlinear and the mobility $\mu$ is not a constant but a function of the vertical and horizontal electric field. And there is also an internal feedback so when the input signal driven into the amplifier is increased, the output is also increased until a point where distortion products can no longer be ignored ( [12], [14]).

No transistor is perfectly linear since the inherent nonlinearity of the diode junctions that comprise many of the active devices found in most amplifiers. The harmonics of the 
output signal are generated by nonlinearities of the MOSFET devices. The major three nonlinear elements of the MOSFET devices are nonlinear transconductance $g_{m}$, the device drain capacitance $C_{d}$ and gate capacitance $C_{g s}([12],[15])$. The real MOSFET devices generate higher order distortion ([12], [16]). Models are used to characterize the nonlinear behavior of a semiconductor device in order to predict the resultant signal properties ([16], [15]).

Choosing the bias points of an RF Power Amplifier can determine the level of performance possible with that PA. The Power Class of the amplification determines the type of bias applied to an RF power transistor. A power class- $A$ of operation is an amplifier that is biased so that the output current flows at all the time. Another way of stating this is that the conduction angle of the transistor is 360 degrees. That is the transistor conducts for the full cycle of the input signal. A class-B power amplifier is an amplifier in which the conduction angle for the transistor is approximately 180 degrees. Same as in class-A power amplifier, the DC bias applied to the transistor determines the class- $\mathrm{B}$ operation. Common configuration of Class-B amplifier is push-pull amplifier. There are other power amplifier classes of operations including class- $C$, class-D, class- $E$ and class-F.

Power amplifiers can be classified in to two categories, linear and nonlinear. Linear power amplifiers preserve amplitude and phase information where as nonlinear power amplifiers only preserve phase information ( [34], [33]). Linear power amplifiers employ transistors as current sources with high impedance. Nonlinear power amplifiers employ transistors as switches with low impedance. Linear power amplifiers can drive both broadband and narrowband loads. Nonlinear power amplifiers usually drive a tuned circuit narrowband load. Models are used to characterize the nonlinear behavior of a semiconductor device in order to predict the resultant signal properties. The simple polynomial approximation is a nonlinear transfer function based upon the Taylor series expansion. Typically the first-order (gain), second-order (squaring) and third-order (cubing) terms are considered ( [16], [15]).

Power amplifier device nonlinearity can be modeled by a polynomial ( [37], [14])

$$
v_{o}(t)=f\left(v_{i}(t)\right)=a_{1} v_{i}(t)+a_{2} v_{i}^{2}(t)+a_{3} v_{i}^{3}(t)+\cdots a_{N} v_{i}^{N}(t)=\sum_{n=1}^{N} a_{N} v_{i}^{N}(t)
$$


Applying a single-tone RF signal to the power amplifier transistor

$$
\begin{gathered}
v_{i}(t)=A_{1} \cos \left(\omega_{o} t+\phi_{1}\right) \\
v_{o}(t)=a_{1} A_{1} \cos \left(\omega_{o} t+\phi_{1}\right)+a_{2} A_{1}^{2} \cos ^{2}\left(\omega_{o} t+\phi_{1}\right)+\cdots+a_{n} A_{1}^{n} \cos ^{n}\left(\omega_{o} t+\phi_{1}\right)
\end{gathered}
$$

Writing out the response $v_{o}(t)$ by performing trigonometric expansion ( [38], [39])

$$
\begin{aligned}
& v_{o}(t)=a_{1} A_{1} \cos \left(\omega_{o} t+\phi_{1}\right)+a_{2} \frac{A_{1}^{2}}{2}-a_{2} \frac{A_{1}^{2}}{2} \cos ^{2}\left(2 \omega_{o} t+2 \phi_{1}\right) \\
& +a_{3} \frac{A_{1}^{3}}{4} \cos ^{2}\left(3 \omega_{o} t+3 \phi_{1}\right)+a_{3} \frac{3 A_{1}^{3}}{4} \cos ^{2}\left(\omega_{o} t+\phi_{1}\right)
\end{aligned}
$$

Table 2.1: One Tone Signal Generated Harmonics

\begin{tabular}{|c||c|}
\hline$a_{1} A_{1} \cos \left(\omega_{o} t+\phi_{1}\right)$ & Linear Gain \\
\hline$a_{2} \frac{A_{1}^{2}}{2}$ & DC Offset (self-bias) \\
\hline$a_{2} \frac{A_{1}^{2}}{2} \cos ^{2}\left(2 \omega_{o} t+2 \phi_{1}\right)$ & Second Harmonic Distortion \\
\hline$a_{3} \frac{A_{1}^{3}}{4} \cos ^{2}\left(3 \omega_{o} t+3 \phi_{1}\right)$ & Third Harmonic Distortion \\
\hline$a_{3} \frac{3 A_{1}^{3}}{4} \cos ^{2}\left(\omega_{o} t+\phi_{1}\right)$ & (AM AM and AM PM) \\
\hline
\end{tabular}

Another method of testing power amplifier linearity is the two-tone method with two closely spaced fundamental signals tones applied to the test amplifier. The amplitude is increased until the third-order cross-product produces a signal above the noise floor ( [34], [33], [36]).

As shown in Figure 2.3 the result of applying two tones to amplifiers that exhibit a degree of nonlinearity is intermodulation distortion (IMD) and third-order harmonics grouped in harmonic zones. As can be depicted from Figure 2.4 that the third order intermodulation products $\left(2 f_{1}-f_{2}\right)$ and $\left(2 f_{2}-f_{1}\right)$ are the main contributor to distortion in that they are very near the fundamental tones and are not filtered out as is the case of the second order intermodulation products $\left(f_{1}-f_{2}, 2 f_{1}, f_{1}+f_{2}\right.$ and $\left.2 f_{2}\right)$ ( [34], [33], [36]). 


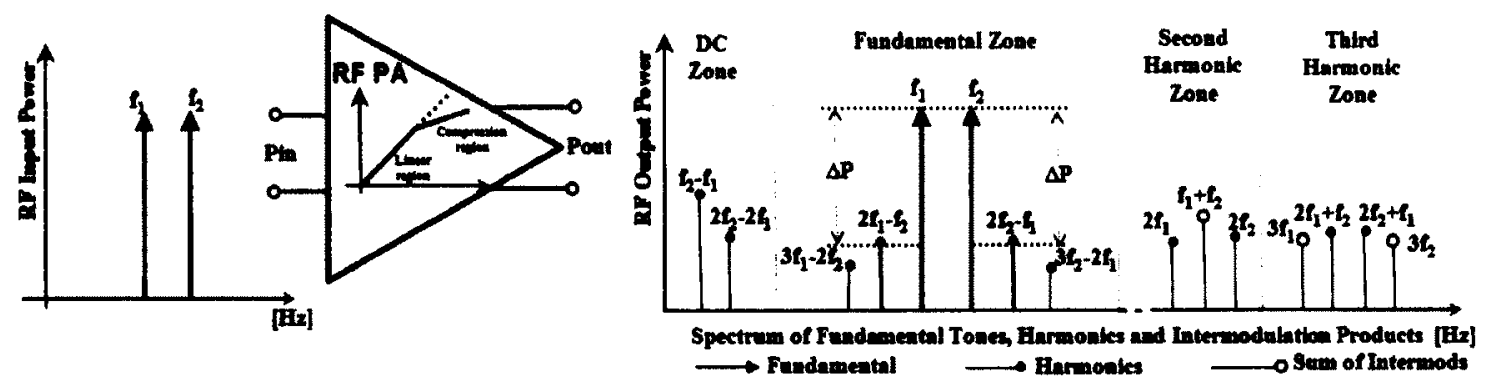

Figure 2.3: Output spectrum of a power amplifier that includes the desired fundamental signals as well as the spurious products created by intermodulation distortion.

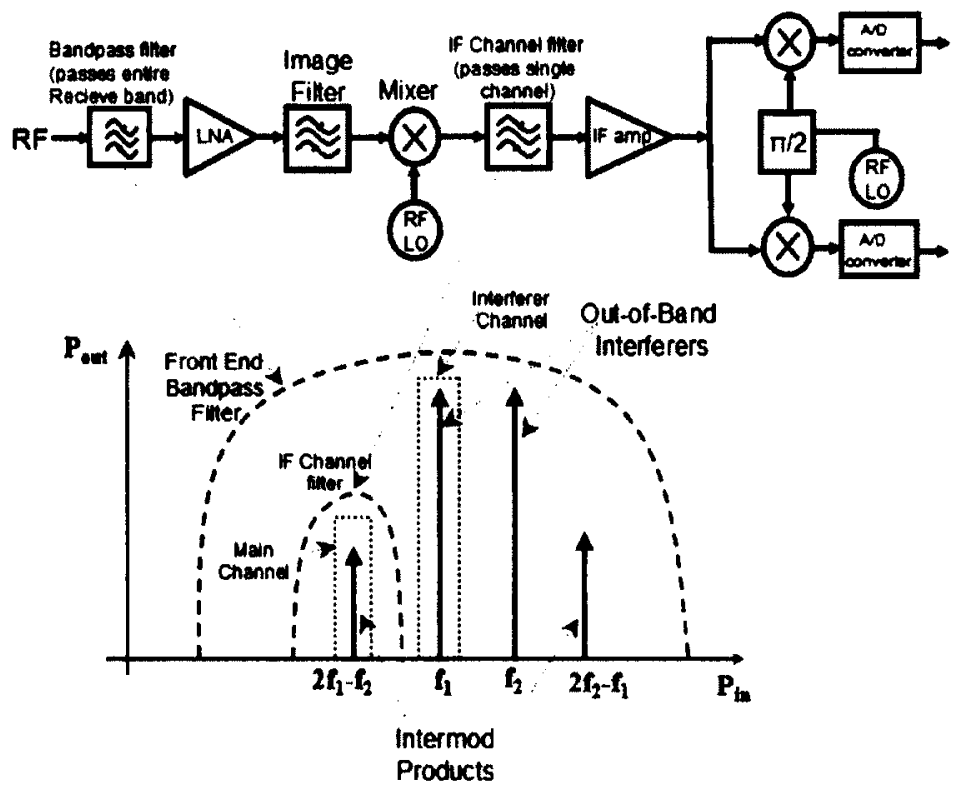

Figure 2.4: Intermodulation products falling in-band. 
Typically the third-order intermodulation distortion product (IM3) are of most concern since distortion products which are far away in frequency from the desired output can be removed by filtering as shown in Figure 2.4 ( [34], [33], [36]).

Applying a two-tone RF signal to the power amplifier

$$
v_{i}(t)=v \cos \left(\omega_{1} t\right)+v \cos \left(\omega_{2} t\right)
$$

The two-tone signal covers the complete dynamic range of the amplifier. The amplifier output is a power series expansion up to the fifth-order is given by [33]

$$
v_{o}=a_{1} v_{i}+a_{2} v_{i}^{2}+a_{3} v_{i}^{3}+a_{4} v_{i}^{4}+a_{5} v_{i}^{5} \ldots
$$

The output voltage with two-tone signal

$$
\begin{aligned}
& v_{o}(t)=a_{1} v\left[\cos \left(\omega_{1} t\right)+\cos \left(\omega_{2} t\right)\right]+a_{2} v^{2}\left[\cos \left(\omega_{1} t\right)+\cos \left(\omega_{2} t\right)\right]^{2} \\
& +a_{3} v^{3}\left[\cos \left(\omega_{1} t\right)+\cos \left(\omega_{2} t\right)\right]^{3}+a_{4} v^{4}\left[\cos \left(\omega_{1} t\right)+\cos \left(\omega_{2} t\right)\right]^{4} \\
& +a_{5} v^{5}\left[\cos \left(\omega_{1} t\right)+\cos \left(\omega_{2} t\right)\right]^{5}
\end{aligned}
$$

Figure 2.5 shows a plot of IM3 versus $P_{\text {in }}$ and $P_{\text {out }}$ versus $P_{\text {in }}$. If the 1:1 slope line of the fundamental Pout and the 1:3 slope line of the third order intermodulation product are extended, they will intersect at a point called IP3, the third-order intercept point. IP3 is an approximation because the slope assumption is not truly valid outside the linear region. The higher the IP3 point the less distortion at higher power levels. In the linear part of Figure 2.5, the Pout versus Pin curve has a slope of 1:1. The P1dB point which is the power where the gain drops by $1 \mathrm{~dB}$ compared to the linear gain was another way to characterize power amplifiers ( [34], [33], [36]).

In the nonlinear region in Figure 2.5 higher efficiency is gained over 50 percent, however distortion is increased significantly. Constant amplitude modulation schemes like AMPS or FM radio use saturated power amplifiers that are more efficient than linear ones ( [34], [33], [36]). 
Table 2.2: Two Tone Signal Generated Harmonics Coefficients

\begin{tabular}{|c||c|c|c|c|c|}
\hline & $a_{1} v$ & $a_{2} v^{2}$ & $a_{3} v^{3}$ & $a_{4} v^{4}$ & $a_{5} v^{5}$ \\
\hline DC & & 1 & & $9 / 4$ & \\
\hline$\omega_{1}$ & 1 & & $9 / 4$ & & $25 / 4$ \\
\hline$\omega_{2}$ & 1 & & $9 / 4$ & & $25 / 4$ \\
\hline $2 \omega_{1}$ & & $1 / 2$ & & 2 & \\
\hline $2 \omega_{2}$ & & $1 / 2$ & & 2 & \\
\hline$\omega_{1} \pm \omega_{2}$ & & 1 & & 3 & \\
\hline \hline $2 \omega_{1} \pm \omega_{2}$ & & & $3 / 4$ & & $25 / 8$ \\
\hline$\omega_{1} \pm 2 \omega_{2}$ & & & $3 / 4$ & & $25 / 8$ \\
\hline \hline $3 \omega_{1}$ & & & $1 / 4$ & & $25 / 16$ \\
\hline $3 \omega_{2}$ & & & $1 / 4$ & & $25 / 16$ \\
\hline $2 \omega_{1} \pm 2 \omega_{2}$ & & & & $3 / 4$ & \\
\hline$\omega_{1} \pm 3 \omega_{2}$ & & & & $1 / 2$ & \\
\hline $3 \omega_{1} \pm \omega_{2}$ & & & & $1 / 2$ & \\
\hline
\end{tabular}

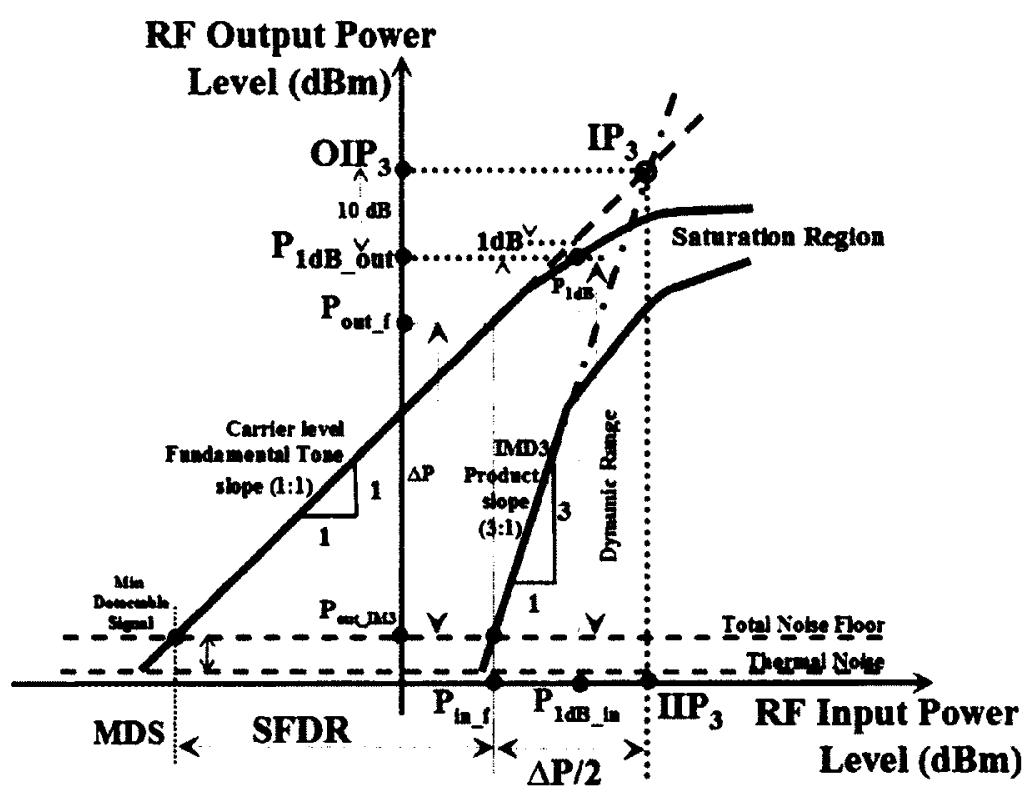

Figure 2.5: Output power versus input power defining third -order intercept point IP3. 


$$
\begin{gathered}
P_{I M 3}=3 \times P_{I N}-2 \times I I P 3(\mathrm{dBm}) \\
O I P_{3}=\frac{3 \times P_{\text {out_f }}-P_{\text {out_IM3 }}}{2}
\end{gathered}
$$

Dynamic range is the signal range in which the signal can still be processed with high quality. It is the signal range whose lower limit is defined by the sensitivity level and whose upper limit is defined by the acceptable maximum level of signal distortion ( [34], [33], [36]). SFDR (spurious free dynamic range) can be found from the two linear equations 2.2.18 and 2.2.19 for the harmonic and third-order intermodulation product where MDS is the minimum detectable signal [34].

$$
S F D R=2 / 3(I I P 3-M D S)
$$

All amplifiers have maximum output power capacity which is called saturation output power. There are a number of ways to specify the nonlinear behavior of a power amplifier. One method of defining amplifier's linearity is third-order intercept point (IP3) ( [34], [33], [36]). This method relies on a figure of merit that is determined by graphical extrapolation of amplifier data taken well below saturation. The IP3 is a theoretical point obtained by extending the two functions until they intersect. If an amplifier was operated at a given level below this third order intercept point then its linear performance was considered adequate. Figure 2.5 is a plot of the output versus input transfer function of the power amplifier whose desired fundamental outputs $\left(f_{1}\right.$ and $\left.f_{2}\right)$ describe a function with a slope of one. The third order intermodulation products are also plotted in Figure 2.5. The output level continues to increase with an increase of input power until a point is reached where output device begins to saturate resulting in a gradual roll-off of output power.

When the actual output power level differs by $1 \mathrm{~dB}$ compared to the ideal output value, the P1dB compression point is reached. In other words, $\mathrm{P} 1 \mathrm{~dB}$ is the output power level point at which the gain is $1 \mathrm{~dB}$ compressed. The $\mathrm{P} 1 \mathrm{~dB}$ is another main power amplifier identification parameter. The higher the intercept point, the better the amplifier is at amplifying large signals ( [34], [33], [36]). However, with variety of new modulation methods, the $\mathrm{P} 1 \mathrm{~dB}$ compression point is not enough for power amplifier performance prediction 
( [34], [33], [36]).

IIP3 can be described by

$$
I P 3_{\mid d B m}=P_{I N \mid d B m}+\frac{\Delta P_{\mid d B}}{2}
$$

IP5 may be determined using the two-tone test as well. Similar to IP3 equation, IP5 can be described by

$$
I P 5_{\mid d B m}=P_{I N \mid d B m}+\frac{\Delta P_{\mid d B}}{4}
$$

\subsubsection{RF Power Amplifier Linearity for Modulated Signals}

The new generation of mobile communication technologies employ linear modulation (QPSK, QAM) and wide bandwidth for increasing bit rate and spectrum efficiency. Therefore the power amplifier is required to process high data rate non-constant envelope signals. For achieving good power efficiency, the power amplifier should work around its compression point however making the output signal distorted nonlinearly ( [40], [41]).

Table 2.3: Different PAR values for different modulated signal types ( [1], [2]).

\begin{tabular}{|c||c|c|}
\hline Modulated Signal Type & Modulation Method & PAR $[\mathrm{dB}]$ \\
\hline CDMA & QPSK & 10 \\
\hline TDMA & ח/4-DQPSK & 3.5 \\
\hline 2-tone & AM & 3 \\
\hline 1-tone & FM & 0 \\
\hline
\end{tabular}

Spectrally efficient modulation schemes in wireless systems non-constant envelope signals have high peak to average power ratio. These modulation techniques require a highly linear PA to process high data rate non-constant envelope signals. In general the higher the data rates the higher the peak to average power ratio (PAR) [2]. High peaks can cause the power amplifier to move toward saturation. This causes intermodulation distortion which generates spectral regrowth as shown in Figure 2.6. Spectral regrowth is a condition that interferes with signals in adjacent frequency bands and can be reduced by using 
power amplifier linearization techniques ( [40], [41]). Table 2.3 shows the different PAR values for different modulated signal types [1]. The PAR is a strong function of the type of modulation. For $10 \mathrm{~dB}$ of PAR that means operating the power amplifier at $10 \mathrm{~dB}$ lower power or power back-off which is not as power efficient [42].

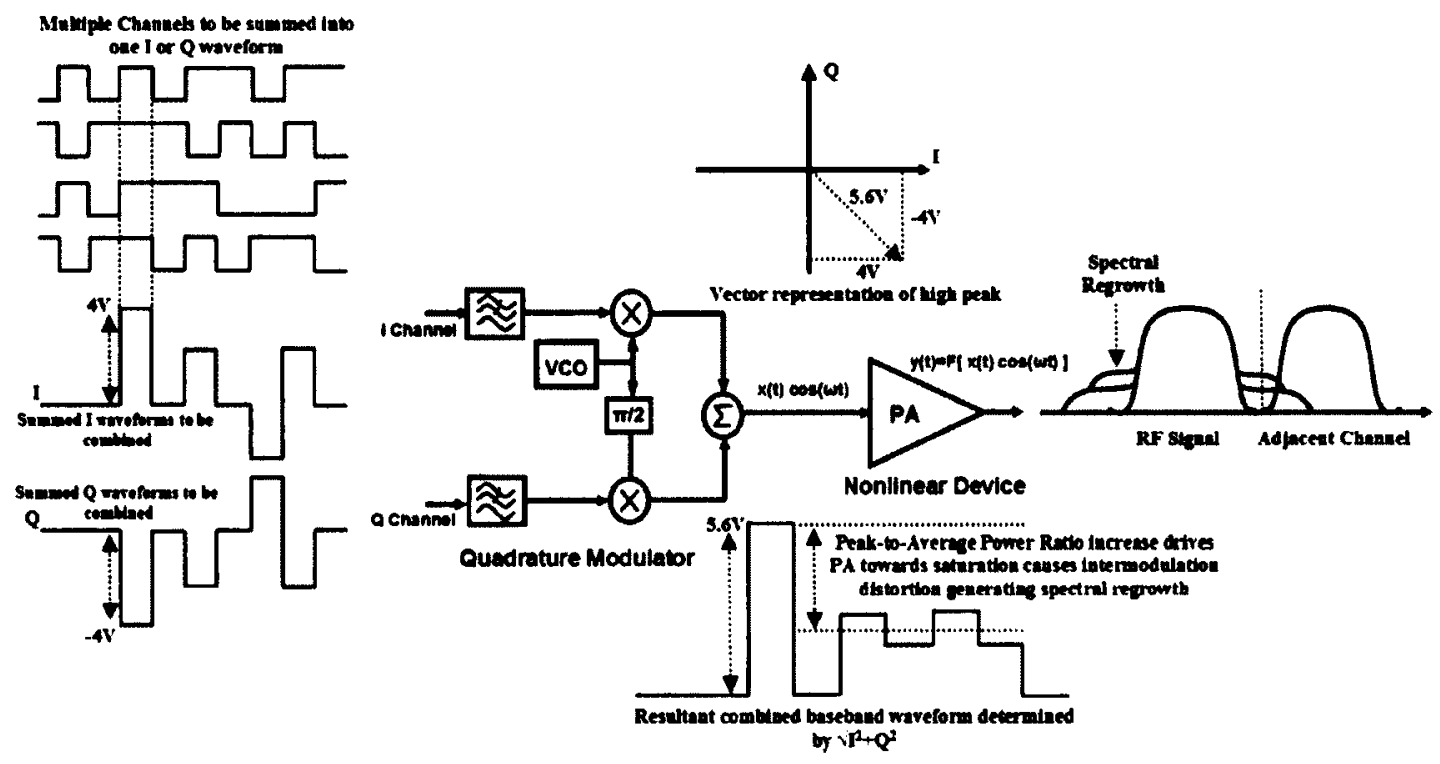

Figure 2.6: RF Power Amplifier Spectral Regrowth.

Power peaks develop in wireless digital communication signal such as CDMA waveforms ( $[2],[40],[41])$. A wireless digital communication signal is generated from a quadrature modulator as shown in Figure 2.6. The waveform is composed of an I waveform and a $\mathrm{Q}$ waveform and these waveforms are the summation of multiple channels. Whenever the channel waveforms simultaneously contain a bit in the same state a high power peak occurs in the summed waveform as shown in Figure 2.6. The I and $Q$ waveforms combine in the quadrature modulator to create an RF waveform ( [40], [41]). The magnitude of the RF envelope is determined by the I squared plus $Q$ squared modulator equation where the squaring of $I$ and $Q$ always results in a positive value. The simultaneous positive peaks in the I and $\mathrm{Q}$ waveforms combine to create a greater peak as shown in Figure 2.6.

Power amplifier nonlinearity effects over modulated signals are out-of-band distortion 
and in-band distortion effects. The out-of-band distortion effects produces a spectrum widening which results in higher ACPR (Adjacent Channel Power Ratio) value. The inband distortion effects produces a constellation distortion which results in higher BER (Bit Error Rate).

\subsubsection{RF Power Amplifier Spectral Regrowth - Out-of-band Dis- tortion}

Adjacent-channel power ratio is the linearity figure-of merit for wireless communication systems employing non-constant envelope modulation techniques such as QAM and $\Pi / 4$ DQPSK ( [40], [41]). These linear modulation techniques, although spectrally efficient, produce modulated carriers with envelope fluctuations. This envelope fluctuation results in signal distortion and spectral spreading when the modulated carrier is passed through a saturated RF power amplifier.

RF power amplifier nonlinear effect impacts the CDMA signal's out of band emission levels. A general mathematical model of a CDMA signal's spectrum, $s(t)$ can be described as ( [43], [44], [40], [41])

$$
s(t)=\sqrt{2} x(t) \cos \left(2 \pi f_{o} t+\theta\right)
$$

where $\mathrm{x}(\mathrm{t})$ is a base-band white Gaussian process with phase $\theta$.

The input-output relationship can be approximated using Taylor polynomial of the input signal for a weak nonlinearity. A general mathematical model of a RF power amplifier can be described as ( [43], [45])

$$
y(t)=F(s(t))=a_{1} s(t)+a_{3} s^{3}(t)
$$

A nonlinear power amplifier transfer function leads to odd-order intermodulation products. These third-order intermodulation products cause distortion. Only the odd-order terms in the Taylor series are considered. The effect of spectra generated by the evenorder terms on the passband are negligible since they are at least $f_{c}$ away from the center of the passband.

Coefficient $a_{1}$ describes the linear gain of the amplifier and $a_{3}$ is the nonlinear coefficient. 
For a linear power amplifier the expression for the $a_{1}$ and $a_{3}$ coefficients can be expressed as ( [43], [45])

$$
a_{1}=10^{\frac{C}{20}}
$$

and

$$
a_{3}=\frac{2}{3} 10\left(\frac{-I P 3}{10}+3 \frac{G}{20}\right)
$$

Coefficient $a_{3}$ is by far the major contributor to the distortion in a power amplifier since it's product appear around carrier frequency $f_{c}$.

The spectrum $P_{y}(f)$ of the RF power amplifier can be expressed as ( [43], [45])

$$
\begin{gathered}
P y(f)=\frac{1}{2 B}\left[P o-6 P_{o}^{2} 10^{-\frac{I P 3}{10}}+9 P_{o}^{3} 10^{-\frac{I P 3}{5}}\right]+\frac{3}{4 B^{3}} P_{o}^{3} 10^{-\frac{I P 3}{3}}\left[6 B^{2}-\left(f-f_{c}\right)^{2}\right],\left|f-f_{c}\right| \leqslant B \\
P y(f)=\frac{3}{8 B^{3}} P_{o}^{3} 10^{-\frac{I P 3}{3}}\left(3 B-\left|f-f_{c}\right|\right)^{2}, B<\left|f-f_{c}\right| \leqslant 3 B \\
P y(f)=0
\end{gathered}
$$

when

$$
3 B<\left|f-f_{c}\right| \leqslant 3 B
$$

Using the results from $P_{y}(\mathrm{f})$, the emission power level within the band $P_{I M 3}$ can be described as ( [46], [45])

$$
P_{I M 3}=\int_{f_{1}}^{f_{2}} P_{y}(f) d f=\frac{1}{8 B^{3}} P_{o}^{3} 10^{-\frac{I P 3}{5}}\left[\left(3 B-f_{1}\right)^{3}-\left(3 B-f_{2}\right)^{3}\right]
$$

IP3 can be expressed in terms of $P_{I M 3}$ as ( [40], [41], [46])

$$
I P 3=-5 \log \left[\frac{P_{I M 3}\left(f_{1}-f_{2}\right) B^{3}}{P_{I M 3}\left[\left(3 B-f_{1}\right)^{3}-\left(3 B-f_{2}\right)^{3}\right]}\right]+22.2 d B m
$$

Equation 2.2.32 describes IP3 in terms of the out-of-band emission power of a CDMA 
signal power amplifier [45]. Figure 2.7 Effects of PA nonlinearity on adjacent channel power ratio. Figure 2.8 shows the predicted output power spectral density (PSD) spectrum for CDMA signal of an RF power amplifier and its distortion effects. A nonlinear power amplifier transfer function leads to odd-order intermodulation products in which these third-order intermodulation products cause spreading of the distortion. These effects lead to spectral regrowth in adjacent channels ([40], [41], [46]).

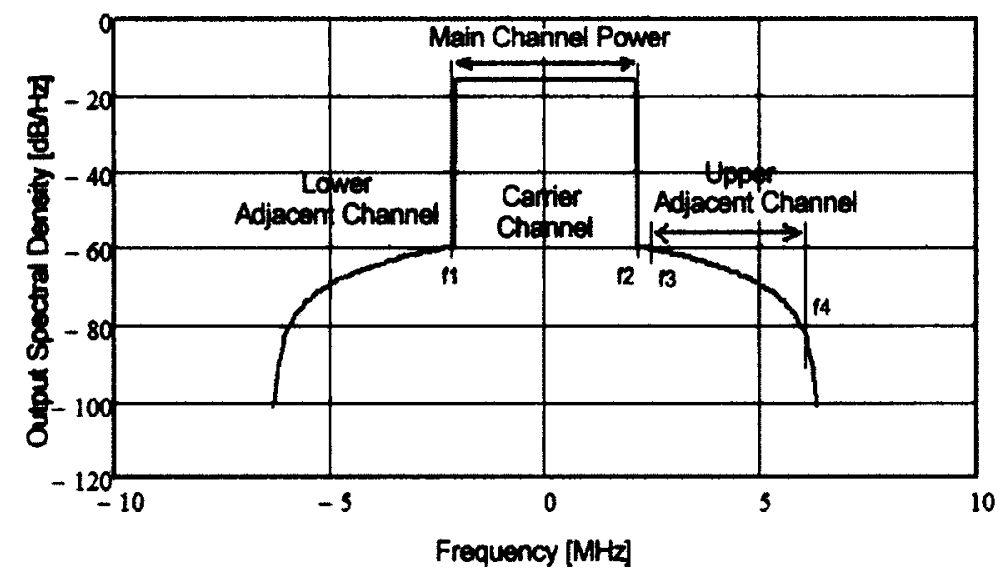

Figure 2.7: Effects of PA nonlinearity on adjacent channel power ratio.

Adjacent Channel Power Ratio (ACPR) is defined as the ratio of the main channel output power to the power in the adjacent channel ( [44], [40]). ACPR helps in determining the amount of signal energy leaked from the main channel to the adjacent channel and is used in testing of CDMA-based communication systems [47].

$$
A C P R=\frac{\int_{\text {adjacent_channel }} P_{\text {out }}(f) \cdot d f}{\int_{\text {main_channel }} P_{\text {out }}(f) \cdot d f}
$$

High crest factor can be defined as ( [40], [41], [46])

$$
\xi=10 \log \left(\frac{P_{\text {peak }}}{P_{\text {average }}}\right)
$$




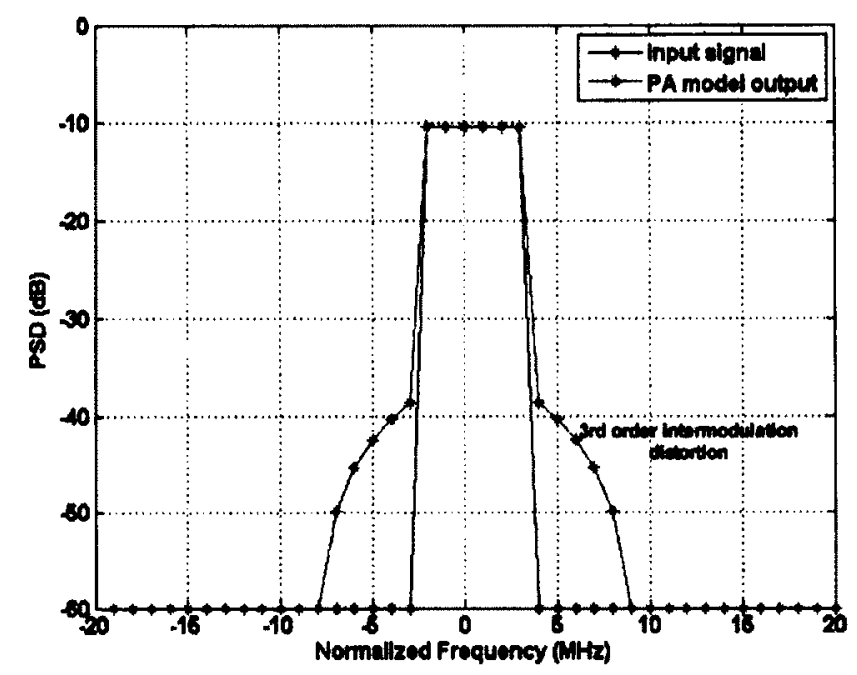

Figure 2.8: Effects of PA nonlinearity on adjacent channel power ratio with third-order distortion.

$$
A C P R=-20.75 d B+1.6 \xi+2\left(P_{\text {in }}-I P 3\right)
$$

RF power amplifier linearization techniques can enhance the overall system response of non-constant modulated signals by reducing ACPR in the PA output power spectral density.

\subsection{Role of RF Power Amplifier Linearization Tech- niques}

This section will discuss techniques for the cancellation of power amplifier distortion that are also known as linearization. Linearization of power amplifiers for radios, using advanced modulation schemes with large peak-to-average ratios, is important in achieving high efficiency as shown in Figure 2.9. Many linearization techniques have been developed to improve power amplifier linearity and to decrease adjacent channel interference ACPR [46]. The basic idea is to run the power amplifier as close to saturation as possible to maximize its power efficiency, and then employ some linearization technique to suppress 


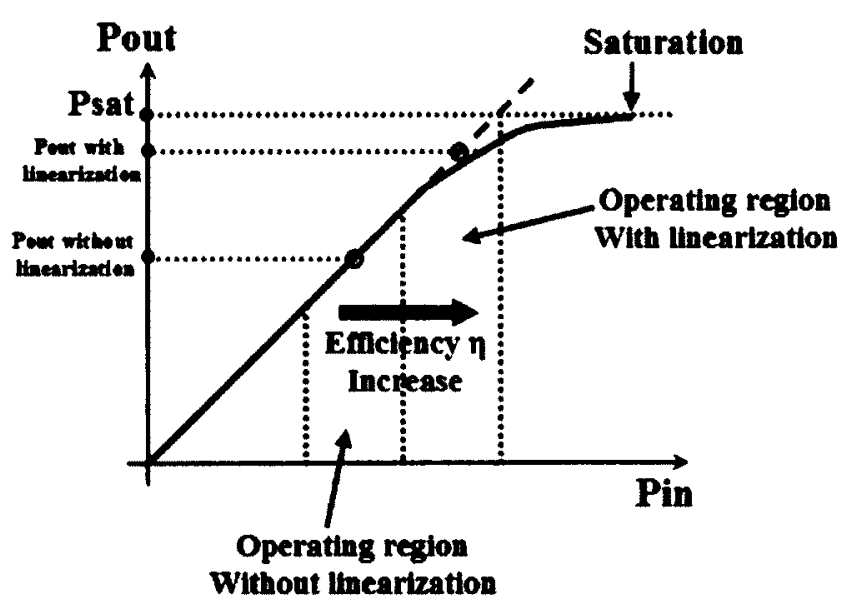

Figure 2.9: Role of Linearization Techniques.

the distortion introduced in this near saturated region [37].

There are many linearization techniques for minimizing power amplifier nonlinear distortion. Linearization can be conducted at either circuit-level or sub-system level. Linearization techniques such as RF power amplifier power Back-off, RF predistortion, feedback and feedforward are often used. Here is a brief review of the major techniques for improving power amplifier linearity [37].

\subsubsection{RF Power Amplifier Power Back-off}

A simple way to improve power amplifier linearity performance is the Back-off operation [37]. To reduce distortion to an acceptable level one must operate the power amplifier at reduced power level (back-off from saturation). The back-off is the distance between the saturated point and the average power level. Increasing the back-off of the power amplifier means that the signal is contained better in the linear range, and thus the effects of nonlinearities are reduced. However, power efficiency is reduced as well. When a power amplifier is driven with decreased input power, the linearity of the power amplifier is improved as shown in Figure 2.10. 


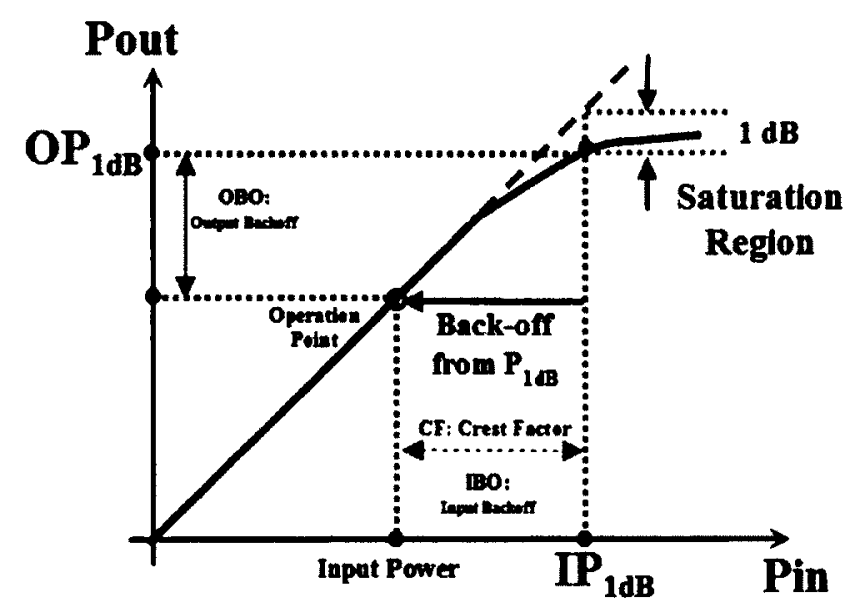

Figure 2.10: RF Power Amplifier Power Back-off.

$$
\text { InputPower }=I P_{1 d B}-I B O
$$

The benefits of the Back-off linearization technique is that it is simple. However, output back-off results in poor efficiency of DC-to-RF power conversion. Since efficiency has a high impact on cellular units talk time, allowances for output back-off have significantly been reduced [37]. So a trade off between efficiency and linearity must be made. Linearization techniques prove to be the best solution in order to improve power amplifiers linearity without having negative impact on efficiency as shown in Figure 2.9.

\subsubsection{RF Power Amplifier Feedforward Linearization}

The feedforward linearization technique was invented by H. S. Black and has since found applications in many communication systems [37] and [48]. The feedforward linearization architecture is shown in Figure 2.11 and it is based on splitting the input signal $x(t)$ into two branches. In the main branch the input signal $x(t)$ is amplified by the main power amplifier yielding the PA output $\mathrm{z}(\mathrm{t})$. In the secondary branch the PA output $\mathrm{z}(\mathrm{t})$ is scaled 


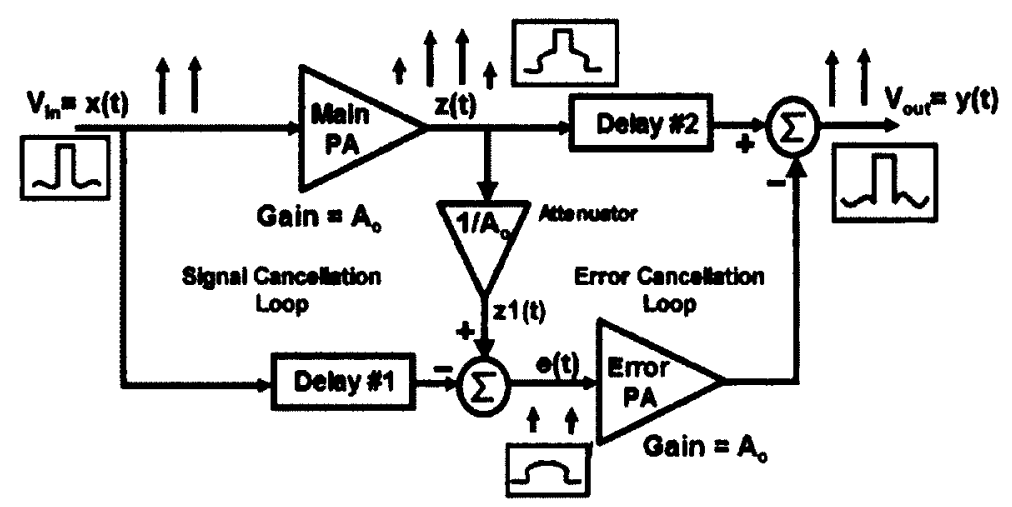

Figure 2.11: RF Power Amplifier Feedforward Linearization.

and compared with the original input $x(t)[37]$.

The resulting error signal $e(t)$ goes through a second PA known as the error PA. After the error signal $e(t)$ is obtained it is amplified and subtracted from the delayed output of the main PA. Since the error signal $e(t)$ is the nonlinear distortion, removing it from the PA output linearizes the PA [37].

The following equations describes the feedforward linearization. Once the PA output $z(t)$ is attenuated to the same level of the input signal $x(t)$ by [37]

$$
z 1(t)=z(t) / A_{0}
$$

then obtaining the distortion by comparing $z 1(t)$ with the input signal $x(t)$

$$
e(t)=x(t)-z 1(t)=x(t)-z(t) / A_{0}
$$

The distortion can be amplified with the auxiliary error PA and subtracted from the original PA output as shown in Figure 2.11

$$
y(t)=z(t)+e(t) \cdot A_{0}=z(t)+\left[x(t)-\frac{z(t)}{A_{0}}\right] \cdot A_{0}=A_{0} \cdot x(t)
$$

Feedforward linearization is stable however it suffers from poor efficiency since an auxiliary error PA is needed. 


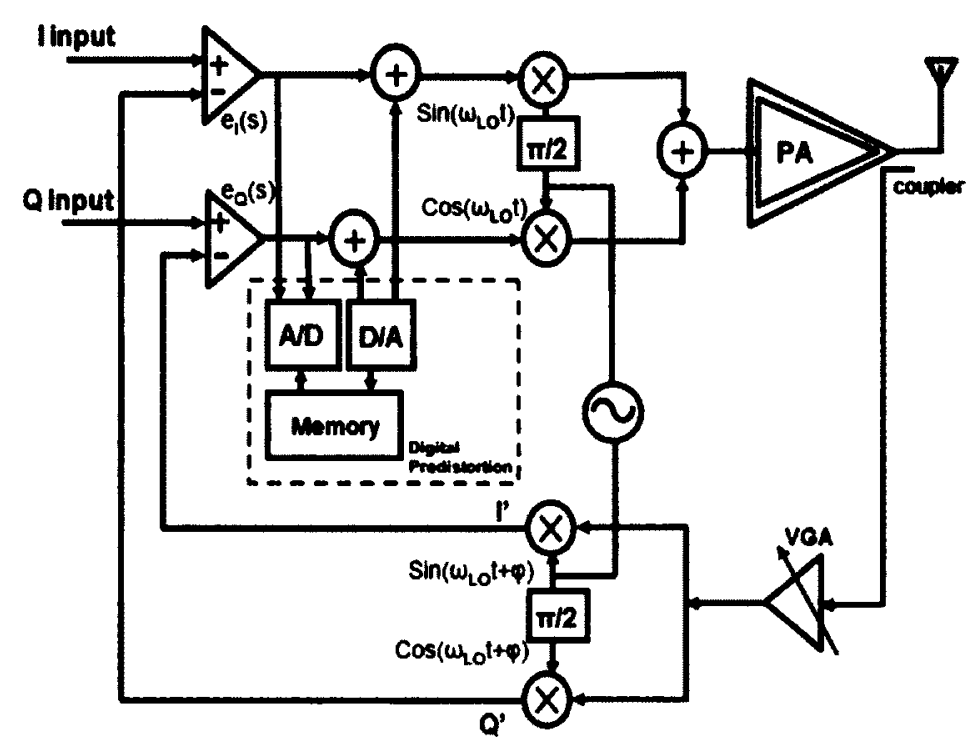

Figure 2.12: RF Power Amplifier Digitally Assisted Cartesian Feedback Linearization.

\subsubsection{RF Power Amplifier Cartesian Indirect Feedback Lineariza- tion}

Many types of feedback linearization techniques exist including cartesian feedback and polar feedback [37], [4], [49]. Cartesian feedback linearization technique is based on feedback control system [4].

In cartesian feedback linearization as shown in Figure 2.12 [2], the distorted PA output is fed back through an I-Q demodulator to build two negative feedback loops [50]. The distorted RF signal from the antenna is split into distorted Cartesian components I' and Q' [37]. The undistorted I and Q signal from the input and the distorted I' and Q' signal from the antenna are fed into differential amplifiers. The differential amplifiers compare two input signals and the amplified error signal $e(s)$ are up-converted to an RF signal using a modulator. Because the feedback technique takes the output of the power amplifier as a reference during the correction process and it overcomes the behavior variation of the 


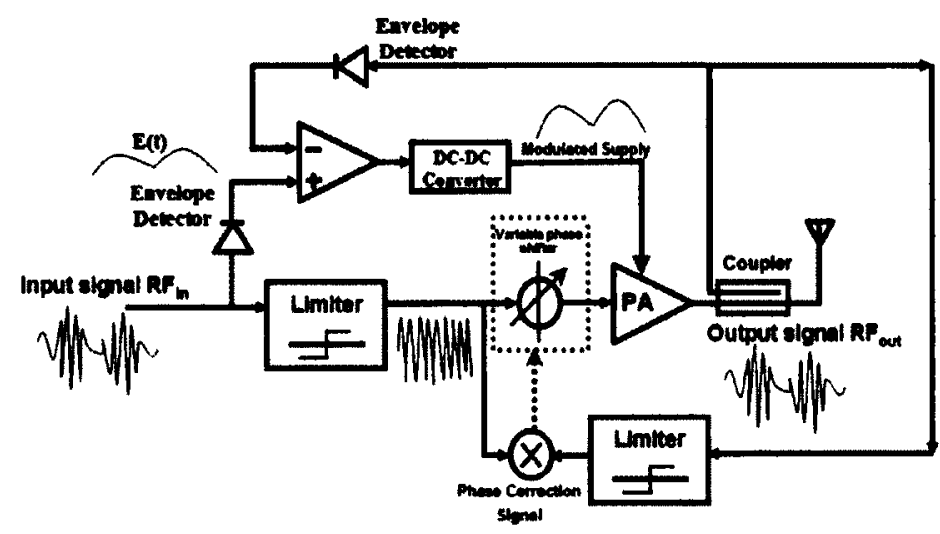

Figure 2.13: RF Power Amplifier Polar Feedback Linearization.

power amplifier [37]. The main objective of the cartesian feedback is to keep feedback phase $\varphi$ aligned with the input signal phase. Cartesian feedback technique is simple and power efficient however it suffers from limited bandwidth ([2], [37], [51]).

\subsubsection{RF Power Amplifier Polar Feedback Linearization}

Like cartesian feedback the polar feedback linearization technique is a baseband feedback scheme ( $[48],[52])$. The polar feedback uses the magnitude and the phase of the PA output signal as feedback signals opposed to the cartesian feedback which uses inphase and quadrature signals ( [37], [53]). It seems more appropriate to use the envelope signal as one of the feedback signals because the distortion (AM/AM and AM/PM) is directly related to this signal ( [48], [52], [37], [53]).

First the output from the PA is down converted to an intermediate frequency as shown in Figure 2.13. Then the envelope of the signal is extracted. This is carried out by mixing the signal with an amplitude limited version of itself [37]. The amplitude of the input signal and the PA signal is subtracted to calculate the amplitude error. This signal is fed into the power regulator of the PA where the amplitude is adjusted. The phase error is calculated by the phase detector and used to adjust the phase of the VCO. This way the phase distortion of the PA is compensated by a phase change in the VCO. Polar feedback linearization technique provides relatively high efficiency since the power amplifier can operate completely nonlinearly [37]. 


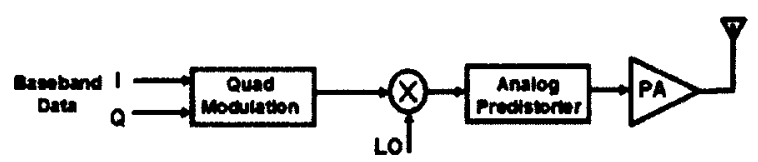

(a)
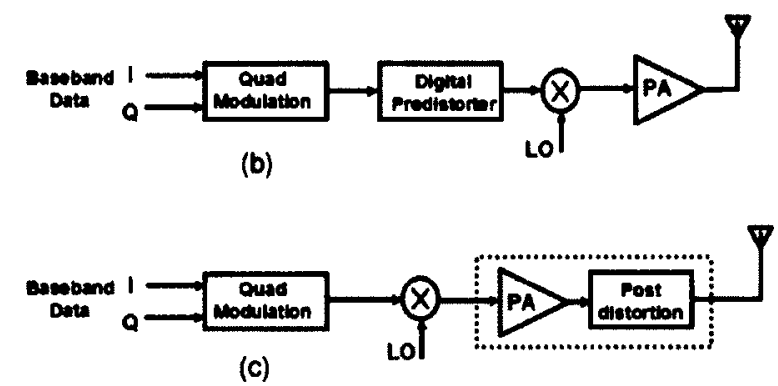

Figure 2.14: RF Power Amplifier RF Predistortion Linearization.

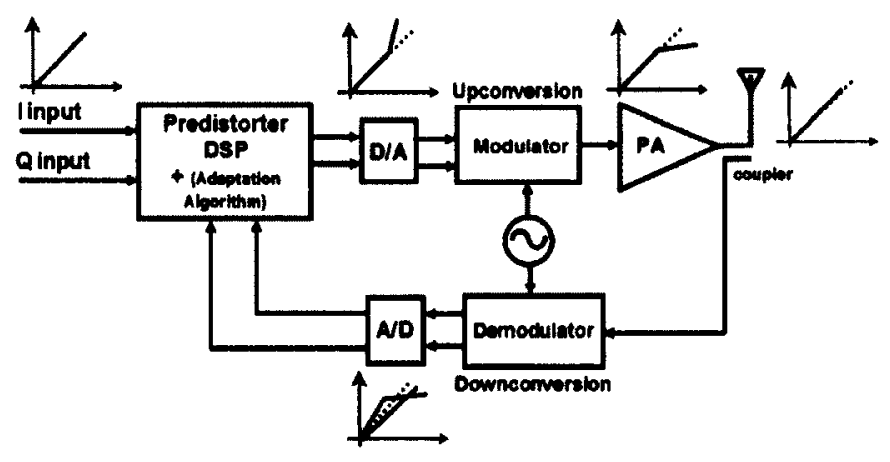

Figure 2.15: RF Power Amplifier Adaptive Digital Predistortion Linearization.

\subsubsection{RF Power Amplifier RF Predistortion Linearization}

RF Predistortion is a popular linearization technique [54], it actively tracks and applies an inverse to the amplifier nonlinearity [10]. If the amplifier exhibits a gain compression the predistortion linearizer is designed to have a gain expansion characteristic. The linearizer can be either active, passive, shunt or series [37]. As input power is increasing it will absorb less power to compensate the power gain roll-off of the following PA. When the input power is decreasing the effect is reversed. The fundamental principle of these predistortion techniques is to adjust the amount of input power [3]. 
An RF predistortion system uses an active or passive analog nonlinear element operating at the radio carrier frequency to generate the predistorted signal as shown in Figure 2.14. IF predistortion implements the predistortion at some intermediate frequency allowing the system to be used at a number of different carrier frequencies [18]. Another implementation of predistortion is baseband predistortion where the inverse transfer function is applied prior to upconversion of the signal.

A possible simplified implementation of adaptive digital predistortion is shown in Figure 2.15 [2]. Adaptive digital predistortion solves the problem of power amplifier variations in RF predistortion. It maintains a dynamically updated model of the power amplifier. Adaptive digital predistortion has an advantage of not suffering from bandwidth limitations incurred by feedback techniques ( [55], [37]). A comparison of different RF power amplifier linearization techniques is shown in Table 2.4 ( [2], [53], [37]).

Table 2.4: Comparison of Different RF Power Amplifier Linearization Techniques.

\begin{tabular}{|c||c|c|c|c|c|}
\hline $\begin{array}{c}\text { Linearization } \\
\text { Technique }\end{array}$ & $\begin{array}{c}\text { Linearization } \\
\text { Performance }\end{array}$ & $\begin{array}{c}\text { Compensation } \\
\text { Bandwidth }\end{array}$ & Cost & Issues & $\begin{array}{c}\text { Control } \\
\text { Applied At }\end{array}$ \\
\hline Feedforward & good & wider & high & low efficiency & Output \\
\hline $\begin{array}{c}\text { Cartesian Indirect } \\
\text { Feedback }\end{array}$ & moderate & narrow & moderate & $\begin{array}{c}\text { reduced gain } \\
\text { stability }\end{array}$ & Input \\
\hline $\begin{array}{c}\text { Analog } \\
\text { RF Predistortion }\end{array}$ & low & wide & low & $\begin{array}{c}\text { reduced } \\
\text { gain }\end{array}$ & Input \\
\hline $\begin{array}{c}\text { Digital } \\
\text { Predistortion }\end{array}$ & moderate & wide & moderate & $\begin{array}{c}\text { easy to control } \\
\text { Depends on DSP }\end{array}$ & Input \\
\hline Polar Feedback & moderate & wide & moderate & reduced gain & Input \\
\hline
\end{tabular}

\subsection{Summary}

In this chapter, RF modulation schemes effect on RF power amplifier nonlinearities was presented. RF power amplifier linearity for non-modulated and modulated signals was presented as well. RF power amplifier spectral regrowth out-of-band distortion and in-band distortion was discussed. A review of various power amplifier RF linearization techniques such as feedforward, cartesian feedback, polar feedback and RF predistortion were discussed. 


\section{Chapter 3}

\section{Distributed Amplification Principles and Transconductor Nonlinearity Compensation}

\subsection{Introduction}

In this chapter, analysis of the distributed amplification principles are presented in this chapter. Different linearized transconductors to compensate for the nonlinearity are also presented.

\subsection{Distributed Amplification Principles}

A distributed amplifier is recognized as one of the most popular broadband amplifier designs. A detailed paper by Ginzton et al. [56] turned Percival's patented concept of distributed amplification into an actual implementation. A high gain-bandwidth product is usually the aim in amplifier design. The gain-bandwidth product is proportional to transconductance over capacitance and is defined as ( [57], [58])

$$
\mathrm{G} \cdot \mathrm{Bw}=\frac{g_{m}}{C}
$$




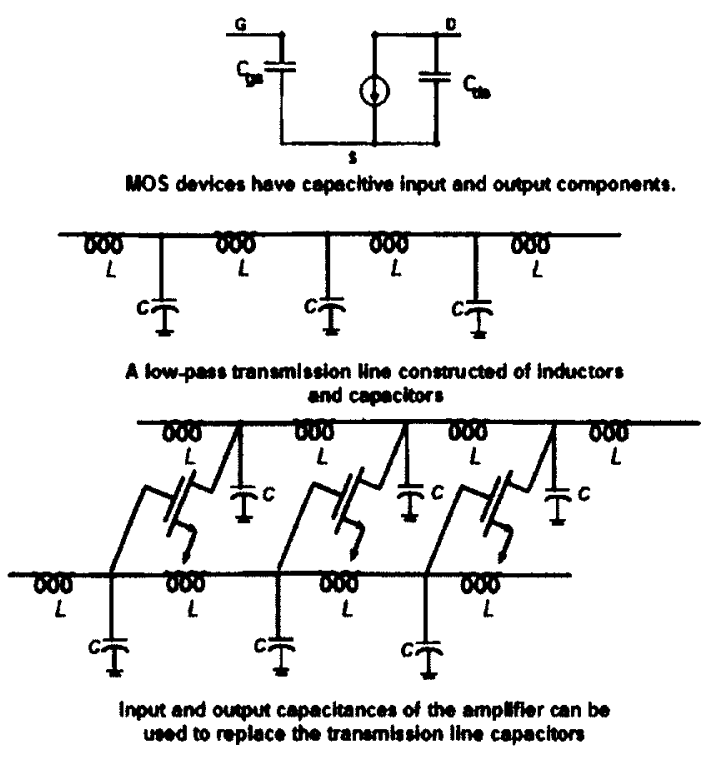

Figure 3.1: A transmission-line constructed of inductors and capacitors coupled with amplification MOS devices.

\subsubsection{Additive Distributed versus Product Cascaded Amplifica- tion}

In a cascaded amplifier system, if the amplifier stages are identical, the overall bandwidth of $\mathrm{N}$ stages $f_{n}$ is related to single stage $3 \mathrm{~dB}$ bandwidth $f_{i}$ by ([59], [16])

$$
f_{n}=0.83 \times \frac{f_{i}}{\sqrt{N}}
$$

where $\mathrm{N}$ is the number of amplifier stages and $f_{n}$ the overall bandwidth of $\mathrm{N}$ stages.

The gain-bandwidth product GBP of a single stage in a cascaded amplifier system is given by $([57],[60])$

$$
G B P=\frac{g_{m}}{2 \pi C_{D}}
$$

where $g_{m}$ is the amplifier transconductance.

The gain $A_{i}$ of a single stage and the GBP in a cascaded amplifier system is given by $[57]$ 


$$
A_{i} \times f_{i}=G B P
$$

The gain $A_{n}$ of $\mathrm{N}$ stage cascaded amplifier system is given by ([60], [59])

$$
A_{n}=\left(A_{i}\right)^{N}=\left(\frac{G B P}{f_{i}}\right)^{N}=\left(\frac{0.83 \times G B P}{\sqrt{N} f_{n}}\right)^{N}
$$

When the number of stages $\mathrm{N}$ is increased in a cascaded amplifier system the gain increases however the bandwidth decreases.
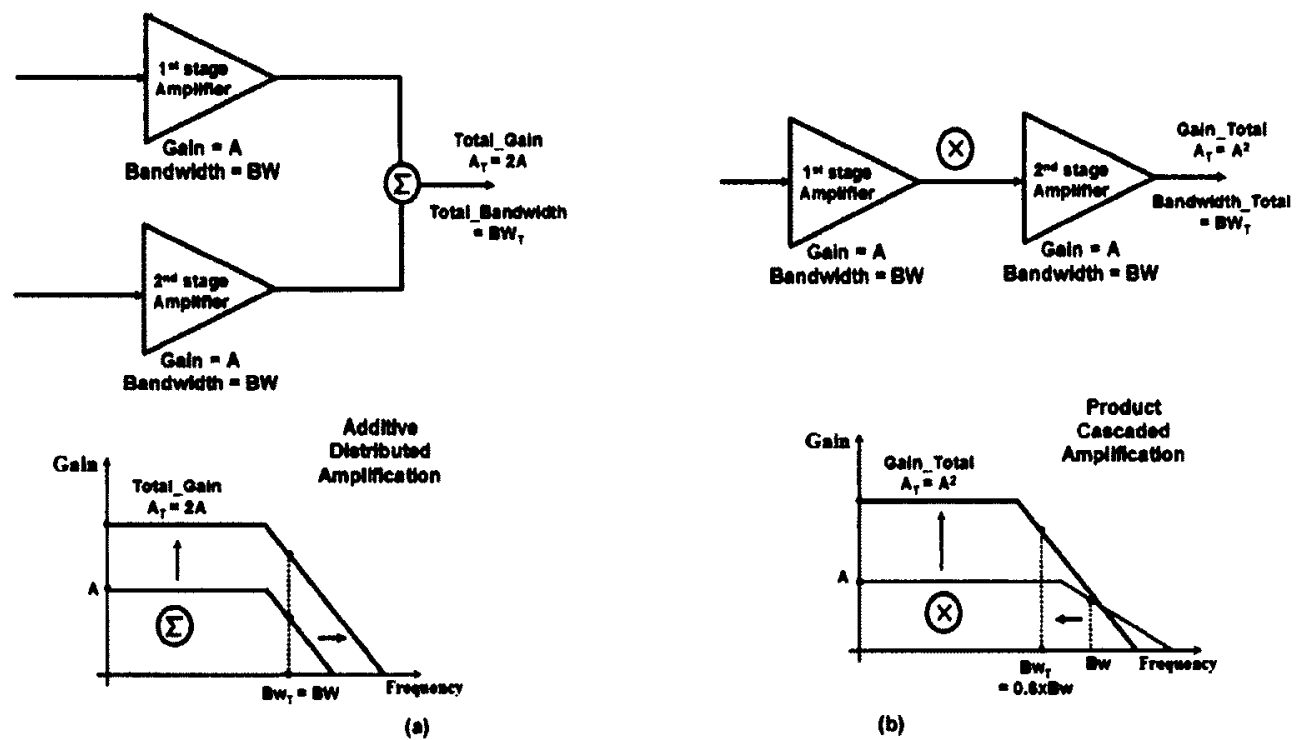

(b)

Figure 3.2: (a) Additive distributed amplification (b) Product cascaded amplification.

For a distributed amplifier the gain is calculated as follows ( [61], [62])

$$
A=\frac{N g_{m} Z_{o d}}{2}
$$

where $Z_{o d}$ is given by

$$
Z_{o d}=\frac{1}{2 \pi C_{d} f_{c}}
$$

For the distributed amplifier the gain is proportional to the number of stages and 
the bandwidth increases as well as can be seen in Figure ??. Distributed amplification allows operation beyond cut-off frequency ( [63], [62]). The gain-bandwidth product of a distributed amplifier is limited by $f_{\max }$ and can be shown to be [57].

$$
A \cdot f_{1 d B}=0.8 f_{\max }
$$

where $f_{1 d B}$ is the frequency at which the gain falls $1 \mathrm{~dB}$ below nominal bandwidth. The maximum available power gain cut-off frequency $f_{\max }$ is given by [57]

$$
f_{\max }=\frac{f_{T}}{2 \sqrt{\frac{R_{i}}{R_{d}}}}
$$

where $f_{T}$ is the unity current gain cut-off frequency and it is given by

$$
f_{T}=\frac{g_{m}}{2 \pi C_{g s}}
$$

where $g_{m}$ is the device transconductance and the $C_{g s}$ is the device gate capacitance.

Combining amplifiers in parallel does not help as it increases the total capacitance. MOS devices have capacitive input and output impedances as depicted in Figure 3.1. However, these capacitances can be incorporated in or counted as capacitors in a transmissionline. A physical structure that guides an electromagnetic wave from place to place is called transmission line [61]. A low-pass transmission-line can easily be constructed of inductors and capacitors and two transmission-lines can be coupled by amplifiers as can be seen in Figure 3.1. Input and output capacitances of the amplifier can be used to replace the transmission-line capacitors [57].

Conventional distributed amplifiers use low-pass $\pi$-sections to form an artificial transmission line topology as can be seen in Figure 3.1. Amplification gain stages are connected so that output currents are combined in an additive manner at the output terminal [62]. The advantages of a distributed amplifier topology are its wide bandwidth, flat gain and compact size. It Provides a good isolation from output to input resulting in a stable amplifier configuration with no oscillation tendency. It also Provides a good input and output match so gain stages can be cascaded. The disadvantages of the distributed amplifier topology are its higher power consumption and lower efficiency. 
The artificial transmission-line topology enables distributed amplifiers to have wider bandwidth [62] as can be seen in Figure 3.3. Transmission-line capacitors, in conjunction with inductors coupled by the transconductance of the FETs provide a propagating medium in which signal waves can travel [64]. If these artificial transmission-lines are well designed, the maximum operating frequency of the amplifier would be limited by the cut-off frequency of the transmission-line [61]

$$
\mathrm{f}_{\mathrm{c}}=\frac{1}{\pi \sqrt{L \bar{C}}}
$$

where $\mathrm{L}$ is the transmission-line lumped series inductance and $\mathrm{C}$ is the lumped shunt capacitance per section.

The transconductance networks isolate the shunt capacitance of the transistors from one another. The capacitances form the integral parts of a filter structure, whose bandwidth is determined by the amount of inductance and capacitance of the filter section. The gain can be increased by introducing more and more sections. We can now trade delay, rather than bandwidth, for gain. As the RF input signal travels down the gate transmission-line, each FET transistor is excited by the traveling power wave and transfers the signals to the drain line through its transconductance [57].

If the phase velocities on the gate and drain lines are equal, then the power signals on the drain line add in the forward direction and an RF output signal is generated ( [65], [66], [67]). The waves traveling in the reverse direction are absorbed by the drain line termination [21]. Artificial transmission-lines are usually modeled by cascading T-sections as shown Figure 3.3 (a). This structure is usually referred to as low-pass Constant-k $\mathrm{T}$-section filter. Constant-k T-section transmission-lines are matched to a load using an $\mathrm{m}$-derived section in order to provide constant Z-impedance over a wider range as can be seen in Figure 3.3 (b). The parameter $\mathrm{m}$ is usually equal to 0.6 and is identified as a practical rule of thumb value. An $\mathrm{m}$-derived section with parameter $\mathrm{m}$ equal to 1 corresponds to a constant-k T-section [62].

The image impedance $Z_{i}$, for a reciprocal symmetric two-port, can be defined as the impedance looking into port 1 or 2 of a general two-port network when the other terminal is also terminated in $Z_{i}$ [68]. To achieve an impedance match over a broad range, the load and source impedance must be transformed into the image impedance $Z_{i}$. The m-derived 


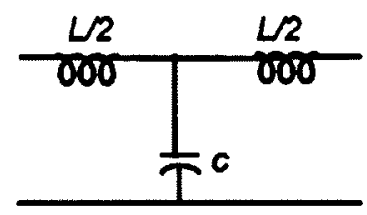

(a) Constant-k T-section

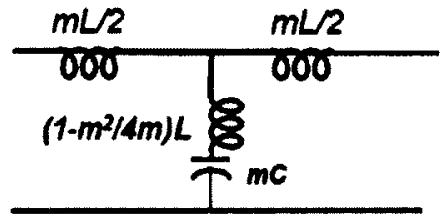

(b) M-derived T-eection

Figure 3.3: (a) Constant-k T-sections (b) m-derived T-sections.

section serves this purpose well. The m-derived impedance matching network provides an improvement to the variation over the broadband frequency. It can also be used to match directly to $Z_{o}=50 \Omega$. The impedance looking into the gate and drain line when transformed by the $\mathrm{m}$-derived section is approximately constant over a broad range of frequencies [57].

\subsubsection{Lumped Constant Delay Line Characteristics}
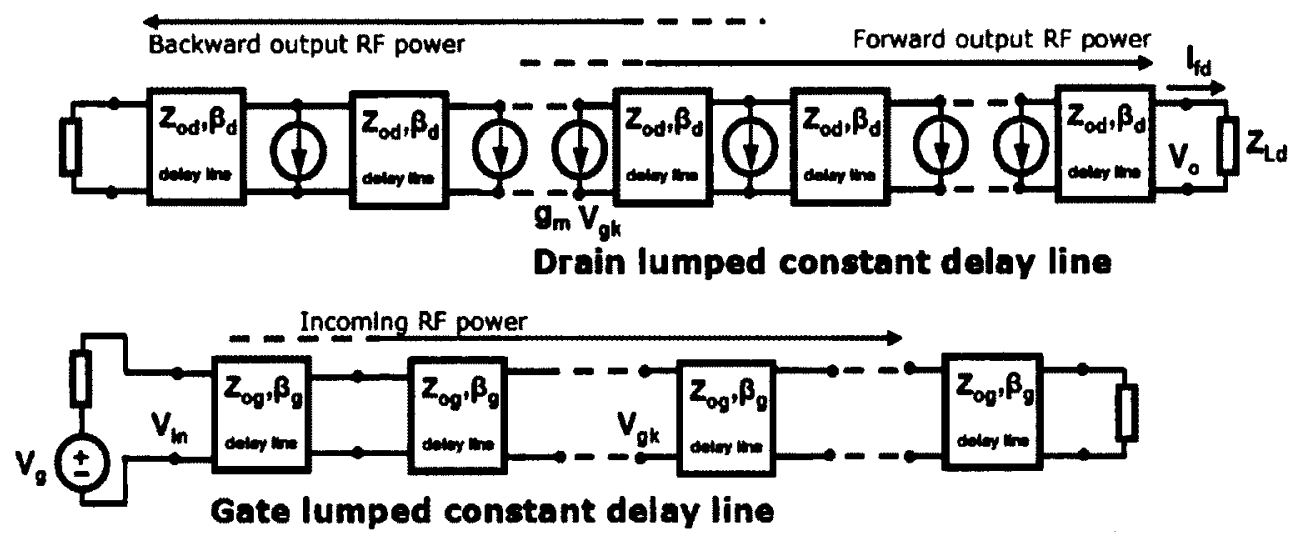

Figure 3.4: Lumped Constant Delay Line Characteristics 
Transmission lines that are designed to intentionally introduce a time delay in the path of an electromagnetic wave are known as delay line. Delay lines are made of lumped elements of $\mathrm{L}$ and $\mathrm{C}$. The inductors are connected in series and the capacitors are connected from the junctions between inductors to the ground. The parasitic $\mathrm{L}$ and the $\mathrm{C}$ from the transistors are used as well. The lumped constant delay line can be considered as a special purpose low pass filter composed of series inductors and shunt capacitors used to delay (phase shift) the input signal by a specified increment of time (degrees). Time delay can be realized with lumped LC delay-lines. The delay of the line, $T_{d}$, is a function of the total inductance and capacitance [62].

The LC artificial transmission line time delay of each section as shown Figure 3.4 can be expressed as inductance and capacitance per section ( [69], [68])

$$
T_{d}=\sqrt{L C}
$$

where $\mathrm{L}$ is the transmission-line lumped inductance and $\mathrm{C}$ is the lumped capacitance per section.

The input and output delay lines are designed to achieve a characteristic impedance of ( [70], [58])

$$
Z o=\sqrt{L / C}
$$

The phase velocity of the LC delay lines can be expressed as [61]

$$
v_{p}=\frac{1}{\sqrt{L C}}
$$

The LC delay line also forms a low-pass filter whose cutoff frequency is [70]

$$
f_{c}=\frac{1}{\pi \sqrt{L C}}
$$

The propagation constant of the LC delay lines is in general a complex quantity and so may be defined as [69]

$$
\gamma=\alpha+j \beta
$$


$\alpha$ is called the attenuation constant and is measured in decibels or nepers per unit length of the transmission line. The propagation constant $\gamma$ is a measure of the phase shift and attenuation per unit length along the line. $\beta$ known as the phase constant is the phase shift per unit length of transmission line and is measured in radians per unit length of this line [61].

$$
\beta=2 \pi / \lambda
$$

where $\lambda$ is the distance along the line corresponding to a phase change of $2 \pi$ radians. The phase constant $\beta$ is expressed as [58]

$$
\beta=\omega \sqrt{L C}
$$

The propagation velocity on the line or phase velocity of a wave is the rate at which the phase of the wave propagates in space. The phase velocity is given in terms of the wavelength (lambda) and period $\mathrm{T}$ or inverse of time delay [57]

$$
v_{p}=\frac{\lambda}{T}=\frac{\omega}{\beta}=\frac{1}{\sqrt{L C}}
$$

The input voltage can be described as [57]

$$
v(t)=V \cos (\omega t)
$$

where $\omega$ is the angular frequency $(\mathrm{rad} / \mathrm{sec})$.

The traveling wave can be described as [58]

$$
v(z, t)=V \cos (\omega t-\beta z)
$$

where $\beta$ is the propagation constant $(\mathrm{rad} / \mathrm{m})$.

If the line is terminated in characteristic impedance, the traveling wave voltage distribution on the line will be reduced due to attenuation [70].

$$
v(z, t)=V e^{-\alpha z} \cos (\omega t-\beta z)
$$

where $\alpha$ is the attenuation coefficient (nepers/meter). 
A phasor can be used to represent the amplitude of a sinusoidal voltage. This phasor does not include any frequency representation [57].

$$
v(z, t)=V e^{-\alpha z} \cos (\omega t-\beta z)=\operatorname{Re}\left(V e^{-\alpha z} e^{-j \beta z} e^{j \omega t}\right)
$$

The voltage wave traveling down the lumped constant delay line, as shown Figure 3.4, is continuously attenuated if the line is terminated in $Z_{o}$ the characteristic impedance of the line. The voltage at the nth section is given by [70]

$$
V_{o}=V_{i n} e^{-\gamma n}
$$

where $V_{\text {in }}$ is a sinusoidally varying input function.

$$
V_{o}=V_{i n} e^{-\alpha n} e^{-j \beta n}
$$

The delays of the input and output lines can be made equal through the selection of propagation constants. Delay mismatches occur when the output currents do not add in phase due to the phase delay in the gate being different than the one in the drain [69].

The gain for a distributed amplifier with $\mathrm{N}$ transistors assuming zero attenuation is given by [57]

$$
\frac{V_{o}}{V_{i n}}=\frac{g_{m} Z_{o}}{2} \sum_{n=1}^{N} e^{-j(n-1 / 2)\left(\beta_{g}-\beta_{d}\right)}
$$

It can be seen that the gain becomes maximum when $\beta_{g}-\beta_{d}=0$

\subsubsection{Lossless Distributed Amplification}

In the case of a lossless lumped cascade of $T$-section, the image impedance and the propagation coefficient are respectively given by [57]

$$
\begin{gathered}
Z_{i}=\sqrt{L / C\left(1-\left(\omega / \omega_{c}\right)^{2}\right)} \\
e^{\gamma}=1-\frac{2 \omega^{2}}{\omega_{c}^{2}}+\frac{2 \omega}{\omega_{c}^{2}} \sqrt{\frac{\omega^{2}}{\omega_{c}^{2}}-1}
\end{gathered}
$$


where $\omega_{c}$ is the line cut-off frequency of the transmission line given by [58]

$$
\omega_{c}=\frac{2}{\sqrt{L C}} .
$$

The characteristic impedance $Z_{o}$ of the lossless artificial transmission-line is given by [61]

$$
Z_{o}=\sqrt{\frac{L}{C}}
$$

where $\mathrm{L}$ and $\mathrm{C}$ are the inductance and capacitance per unit length of the transmission-line. The artificial transmission-line has a cut-off frequency $f_{c}$ given by [58]

$$
f_{c}=\frac{1}{\pi \sqrt{L C}}
$$

The bandwidth is determined by the input capacitance of the transistor and the inductance of the transmission-line yielding a wider bandwidth than that of lumped element circuits. The $C_{g s}$ and $C_{d s}$ of MOS device capacitances are absorbed into the transmissionline.

To get a better understanding of the CMOS DA operation, it useful to consider the wave propagation behavior along the lossless transmission line. Assuming ideal transistors with no parasitics, the common-source amplifier output drain current on the drain line is given by $([16],[67])$

$$
i_{d}=g_{m} \cdot v_{g s}
$$

The RF input signal is fed into the gate line, where it excites the FET transistors along the gate line as it propagates, until it reaches the gate line termination, where it is ideally dissipated without reflections [44].

Assuming gate and drain line have equal propagation velocity and zero loss, $\beta_{g}=\beta_{d}$ and $\alpha=0$ for the lossless condition, where $\alpha$ is defined as attenuation factor and $\beta$ is also known as phase constant ( [13], [71]).

An input wave $v_{g}$ propagating on a lossless line can be expressed as in (3.2.33) ( [41], [58]), where $v_{g}$ appears at the transistor inputs as $v_{g s}$ and half of $V_{s}$ being the amplitude of the input wave that flows into the terminating gate resistance [62]. 


$$
v_{g}(z)=\frac{V_{s}}{2} \cdot e^{-j \beta z}
$$

An input wave propagating along the gate line to the output on the drain line is given by $[61]$

$$
v_{g n}=\frac{V_{s}}{2} \cdot e^{-(n-1) j \beta_{g} \ell_{g}}
$$

where $\mathrm{n}=1$ when it is the first stage, implies there is no delay. The output drain current and output voltage relationship is given in equation (3.2.35) [62]

$$
v_{o}=I_{d} \cdot Z_{d}
$$

The output drain current across the n-th FET, $I_{d}$, can be expressed as ( [58], [48])

$$
I_{d}=\frac{1}{2} \sum_{n=1}^{N} i_{d n}\left(e^{-(N-n)}\right)^{j \beta_{d} \ell_{d}}
$$

where $n=N$ represents the last stage [61]

$$
i_{d n}=g_{m} \cdot v_{g s}
$$

Substituting equation (3.2.34) and (3.2.36) into (3.2.37) we get ( [58], [48])

$$
\begin{gathered}
I_{d}=-\frac{g_{m}}{4} \cdot V_{s} \cdot \sum_{n=1}^{N}\left(e^{-(n-1)}\right)^{j \beta_{g} \ell_{g}} \cdot\left(e^{-(N-n)}\right)^{j \beta_{d} \ell_{d}} \\
I_{d}=-\frac{g_{m}}{4} \cdot V_{s} \cdot e^{-N j \beta_{d} \ell_{d}} \cdot e^{j \beta_{g} \ell_{g}} \cdot \sum_{n=1}^{N}\left(e^{-n j}\right)^{\left(\beta_{g} \ell_{g}-\beta_{d} \ell_{d}\right)}
\end{gathered}
$$

Each FET injects current into the output line as seen in Figure 3.5, if the phase constant of both transmission-lines are equal, the currents add constructively at the output, while out-of-phase signals are dissipated on the drain termination [58]. If under normal operating conditions, the waves in the gate and drain lines are synchronized [72]

$$
\beta_{g} \ell_{g}=\beta_{d} \ell_{d}=\beta \ell
$$




$$
I_{d}=-\frac{g_{m}}{4} \cdot V_{s} \cdot e^{-N j \beta \ell} \cdot e^{j \beta \ell} \cdot N
$$

In the case of ideal lossless constant-k lines, the expression for the power gain of $\mathrm{N}$ section distributed amplifier is thus given by [58]

$$
G=\frac{P_{\text {out }}}{P_{\text {in }}}=\frac{\frac{1}{2}\left|I_{d}\right|^{2} Z_{d}}{\left|v_{g}\right|^{2} / 4 Z_{g}}=\frac{g_{m}^{2} Z_{d} Z_{g} N^{2}}{4}
$$

For the ideal lossless transmission line case the gain increases relative to $N^{2}$ as can be seen from (3.2.42), and the bandwidth is infinite.

\subsubsection{Lossy Distributed Amplification}

In the presence of attenuation, the gate signal decays as it propagates down the line. Hence, there will be a point at which the gain added by an additional device will not overcome the losses induced by the extra section in the gate and drain lines. The reason for this is that the devices added are not driven sufficiently to overcome the losses in the drain line cause of high signal attenuation.

A transmission-line can be constructed by a cascaded T-section structure with a characteristic impedance of ( [57], [63])

$$
Z_{O T}=\sqrt{\frac{L}{C}} \frac{1}{\left(1-\left(\frac{\varpi}{\varpi c}\right)^{2}\right)^{\frac{3}{2}}} \sqrt{\left(1-\left(\frac{\varpi}{\varpi_{r}}\right)^{2}\right)^{2}-\left(\frac{\varpi}{\varpi_{c}}\right)^{2}}
$$

where $\omega_{c}=\frac{2}{\sqrt{L C}}$ is the cut-off frequency and $\omega_{r}=\frac{1}{\sqrt{L C_{d}}}$ is the drain line inductor selfresonance frequency. The presence of gate resistance $r_{g}$ and drain resistance $r_{d s}$ affects the frequency variation of the transmission-line characteristic impedance $Z_{O T}$.

Figure 3.5 shows an equivalent gate line circuit.

A common-source amplifier gate resistance $R_{i}$ in series with parasitic capacitance $C_{g s}$ introduces a frequency dependent attenuation per line section [58]. The impedance of the gate line, shown in Figure 3.6, can be approximated as ( [72], [57])

$$
Z=j \omega L_{g}
$$




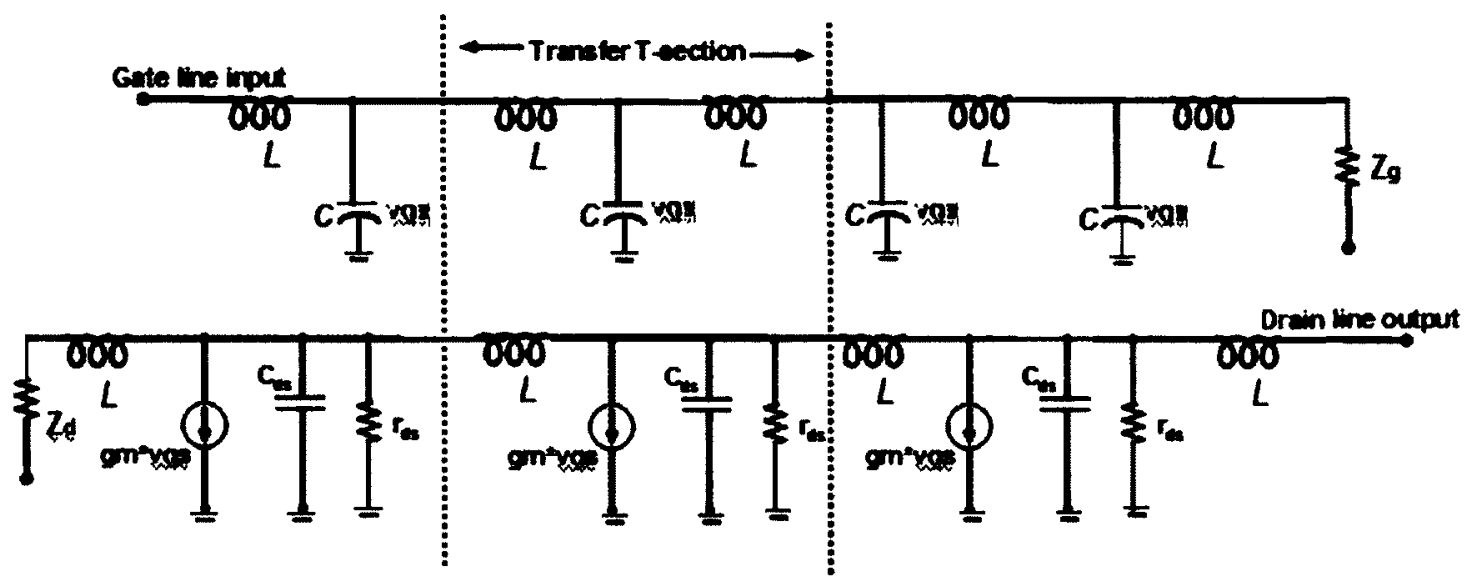

T-sections structure of gate and drain lines of the distributed amplifier.

Figure 3.5: T-sections structure of gate and drain lines of the distributed amplifier.

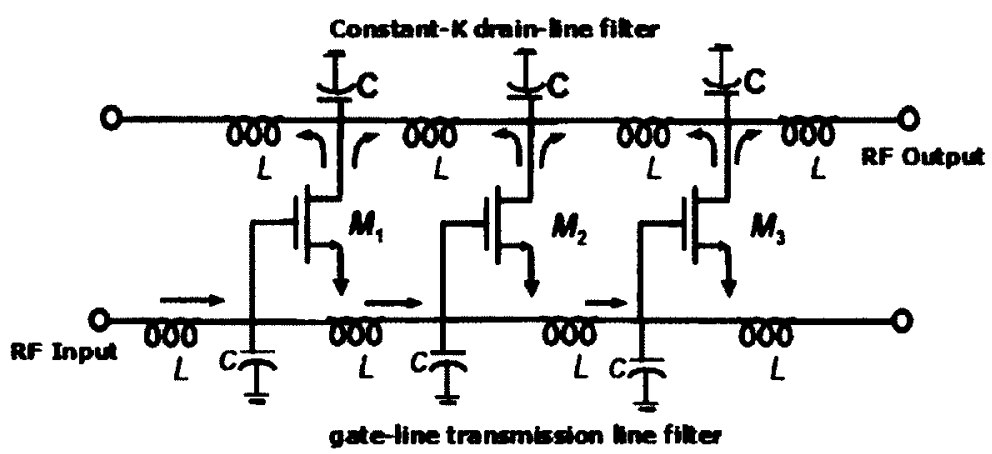

Figure 3.6: Gate and drain transmission lines of a CMOS distributed amplifier. 
where $L_{g}$ is the per-unit-length inductance of the gate line as shown in Figure 3.5.

The propagation constant of the gate line, $\gamma_{g}$ can be expressed as [13]

$$
\gamma_{g}=\alpha_{g}+j \beta_{g}=\sqrt{Z \cdot Y}
$$

where the gate transmission-line equivalent impedance $\mathrm{Z}$ and admittance $\mathrm{Y}$ parameters are given by [13]

$$
\begin{gathered}
Y=j w C g+\frac{j w C_{g s / h_{g}}}{1+j w R_{i} C_{g s}} \\
Z_{g}=\sqrt{\frac{Z}{Y}} \cong \sqrt{\frac{L_{g}}{C_{g}+C_{g s / 1}}}
\end{gathered}
$$

Substituting (3.2.46) and (3.2.47) into (3.2.45) we get the propagation constant of the gate line as follows

$$
\begin{gathered}
\gamma_{g}=\sqrt{j \omega L_{g}\left(j \omega C_{g}+\frac{j \omega C_{g s} / l_{g}}{1+j \omega R_{i} C_{g s}}\right)} \\
\gamma_{g} \cong \sqrt{-\omega^{2} L_{g}\left(C_{g}+C_{g s}\left(1-j \omega R_{i} C_{g s}\right) / l_{g}\right)}
\end{gathered}
$$

where $L_{g}$ is the per-unit-length inductance of the gate line.

The equivalent drain line circuit is shown in Figure 3.5. The impedance of the drain line can be approximated as ( [72], [57])

$$
Z=j \omega L_{d}
$$

where $L_{d}$ is the per-unit-length inductance of the drain line.

The propagation constant of the drain line, $\gamma_{d}$ can be expressed as [13]

$$
\gamma_{d}=\alpha_{d}+j \beta_{d}=\sqrt{Z \cdot Y}
$$

where the drain transmission-line equivalent impedance $Z$ and admittance $Y$ parameters are given by [13] 


$$
Y=\frac{1}{R_{d s} l_{d}}+j \omega\left(C_{d}+C_{d s / l_{d}}\right)
$$

where $R_{d s}$ is the shunt resistance of the MOSFET and $\omega$ is the operating frequency ( [72], [63])

$$
Z_{d}=\sqrt{\frac{Z}{Y}} \cong \sqrt{\frac{L_{d}}{C_{d}+C_{d s} h_{d}}}
$$

Substituting (3.2.52) and (3.2.53) into (3.2.51) we get the propagation constant of the drain line as follows

$$
\gamma_{d}=\sqrt{j \omega L_{d}\left(\frac{1}{R_{d s} l_{d}}+j \omega\left(C_{d}+C_{d s}\right) / l_{d}\right)}
$$

Assuming the $j \omega\left(C_{d}+C_{d s} / l_{d}\right)$ term dominates in (3.2.54), the propagation constant of the drain line, $\gamma_{d}$ can be expressed as

$$
\gamma_{d} \cong \frac{Z_{d}}{2 R_{d s} l_{d}}+j \omega \sqrt{l_{d}\left(C_{d}+C_{d s} / l_{d}\right)}
$$

The output drain current with the lossy condition can be approximated as follows [72]

$$
\begin{gathered}
I_{d}=-\frac{g_{m}}{4} \cdot V_{s} \cdot e^{-N \gamma_{d} \ell_{d}} \cdot e^{\gamma_{g} \ell_{g}} \cdot \sum_{n=1}^{N}\left(e^{-n}\right)^{\left(\gamma_{g} \ell_{g}-\gamma_{d} \ell_{d}\right)} \\
I_{d}=-\frac{g_{m} V_{s}}{4} \cdot \frac{e^{-N \gamma_{g} \ell_{g}}-e^{-N \gamma_{d} \ell_{d}}}{e^{-\gamma_{g} \ell_{g}}-e^{-\gamma_{d} \ell_{d}}}
\end{gathered}
$$

When losses are incorporated in the analysis, the power gain can be determined as, assuming unilateral devices [72]

$$
G=\frac{g_{m}^{2} \cdot Z_{d} \cdot Z_{g}}{4} \cdot \frac{\left(e^{-N \alpha_{g} \ell_{g}}-e^{-N \alpha_{d} \ell_{d}}\right)^{2}}{\left(e^{-\alpha_{g} \ell_{g}}-e^{-\alpha_{d} \ell_{d}}\right)^{2}}
$$

where $\alpha_{g}$ and $\alpha_{d}$ are the attenuation per section in Nepers on the gate and drain line.

$$
e^{-\alpha_{g} \ell_{g}}-e^{-\alpha_{d} l_{d}} \approx \alpha_{g} l_{g}-\alpha_{d} l_{d}
$$

Equation (3.2.58) indicates that the gain is no longer a monotonic function of $\mathrm{N}$, the 
number of DA gain stage sections, but peaks at a finite $N$. In other words, adding an extra stage will only improve gain if the product of $\frac{g_{m} \cdot V_{g o}(N+1) \cdot Z_{d}}{2}$ is greater than $\left|e^{\gamma_{d} \ell_{d}}\right|$, where $\frac{g_{m} \cdot V_{Q d}(N+1) \cdot Z_{d}}{2}$ is the extra gain and $e^{\gamma_{d} \ell_{d}}$ is the attenuation on the drain line. Therefore, the loss due to the extra transmission-line exceeds the gain of the $N^{\text {th }}$ FET. Eventually the polynomial additive increase in DA gain due to the extra stage will not outperform the exponential loss.

By taking the derivative of the gain function in equation (3.2.58), we can then find the optimum number of CMOS gain cells for maximum gain [72]

$$
N_{o p t}=\frac{\ln \left(\alpha_{g} \ell_{g} / \alpha_{d} \ell_{d}\right)}{\alpha_{g} \ell_{g}-\alpha_{d} \ell_{d}}
$$

where $\alpha_{g}$ and $\alpha_{d}$ are the attenuation per section in Nepers on the gate and drain line. We also notice that the gain is not flat with frequency due to frequency dependence of $\alpha_{g}$ and $\alpha_{d}$.

An increase in number of sections, $n$, increases gain linearly. However, line losses and parasitics prevent an infinite increase in DA gain stages. The attenuation on the gate line will drive the input signal to negligibly small values and adding further stages will increase the attenuation on the drain line.

The expression for the forward power gain of a DA is given by [72]

$$
G_{f o r}=\frac{g_{m}^{2} Z_{o g} Z_{o d} \sinh ^{2}\left[\frac{n}{2}\left(\alpha_{d}-\alpha_{g}\right)\right] e^{-n\left(\alpha_{d}+\alpha_{g}\right)_{/ 2}}}{4\left[1+\left(\varpi / \varpi_{g}\right)^{2}\right]^{1 / 2}\left[1-\left(\varpi / \varpi_{g}\right)^{2}\right] \sinh ^{2}\left[\frac{1}{2}\left(\alpha_{d}-\alpha_{g}\right)\right]}
$$

where $Z_{o g}$ and $Z_{o d}$ are the characteristic impedance of the gate and drain lines, respectively, $\alpha_{g}$ and $\beta_{g}$ are the attenuation and phase shift per section on the gate line, $\alpha_{d}$ and $\beta_{d}$ are the attenuation and phase shift per section on the gate line and $\omega_{g}$ is the gate cut-off frequency. In order to maximize the forward gain and minimize the ripple, $\alpha_{g}$ would be set equal to $\alpha_{d}$. As the number of sections increases, the signal level on the gate line is attenuated and so is the power to the drain line. The loss in the drain line includes an exponential factor that is a function of the per section drain line attenuation.

The reverse gain in a DA can be written as ([19], [20]) 


$$
G_{\text {rev }}=\frac{g_{m}^{2} Z_{o g} Z_{o d} e^{-n\left(\alpha_{d}+\alpha_{g}\right)}\left(\cosh \left(n\left(\alpha_{d}-\alpha_{g}\right)\right)-\cos 2 n \beta\right)}{4\left[1+\left(\varpi / \varpi_{g}\right)^{2}\right]^{1 / 2}\left[1-\left(\varpi / \varpi_{c}\right)^{2}\right]\left(\cosh \left(\alpha_{d}+\alpha_{g}\right)-\cos 2 \beta\right)}
$$

The DA isolation is dependent on $G_{\text {rev }}$. The directivity can be derived as [19]

$$
D=10 \log \frac{G_{\text {for }}}{G_{\text {rev }}}=10 \log \left[\left(\frac{\sin \frac{n}{2}\left(\theta_{d}-\theta_{g}\right)}{\sin \frac{1}{2}\left(\theta_{d}-\theta_{g}\right)}\right)^{2}\left(\frac{\sin \frac{1}{2}\left(\theta_{d}+\theta_{g}\right)}{\sin \frac{n}{2}\left(\theta_{d}+\theta_{g}\right)}\right)^{2}\right]
$$

\subsection{Transconductor Gain Cells for Fully-Differential Distributed Amplifiers}

MOSFET devices can be modeled by nonlinear current and nonlinear capacitance that depend on the device voltages ( [12], [13]). These nonlinear sources will give rise to distortion when driven with a modulated signal. The real MOSFET device output impedance is nonlinear and the mobility $\mu$ is not a constant but a function of the vertical and horizontal electric field. We may bias the MOSFET device where the device behavior is more exponential. And there is also an internal feedback so when the input signal driven into the amplifier is increased, the output is also increased until a point where distortion products can no longer be ignored ( [12], [14]).

No transistor is perfectly linear since the inherent nonlinearity of the diode junctions that comprise many of the active devices found in most amplifiers. The harmonics of the output signal are generated by nonlinearities of the MOSFET devices. The major three nonlinear elements of the MOSFET devices are nonlinear transconductance $g_{m}$, the device drain capacitance $C_{d}$ and gate capacitance $C_{g s}[15]$. The real MOSFET devices generate higher order distortion [16]. Models are used to characterize the nonlinear behavior of a semiconductor device in order to predict the resultant signal properties [15].

External linearization circuitry can be added to the amplifier, to compensate for the nonlinearity ( [36], [73]). This allows the nonlinear amplifier to be used to amplify signals utilizing spectrally efficient linear modulation techniques without causing interference.

In radio transceivers, a differential pair transconductor is often used to convert the RF input voltage signal to a current that is either amplified or frequency translated. External 


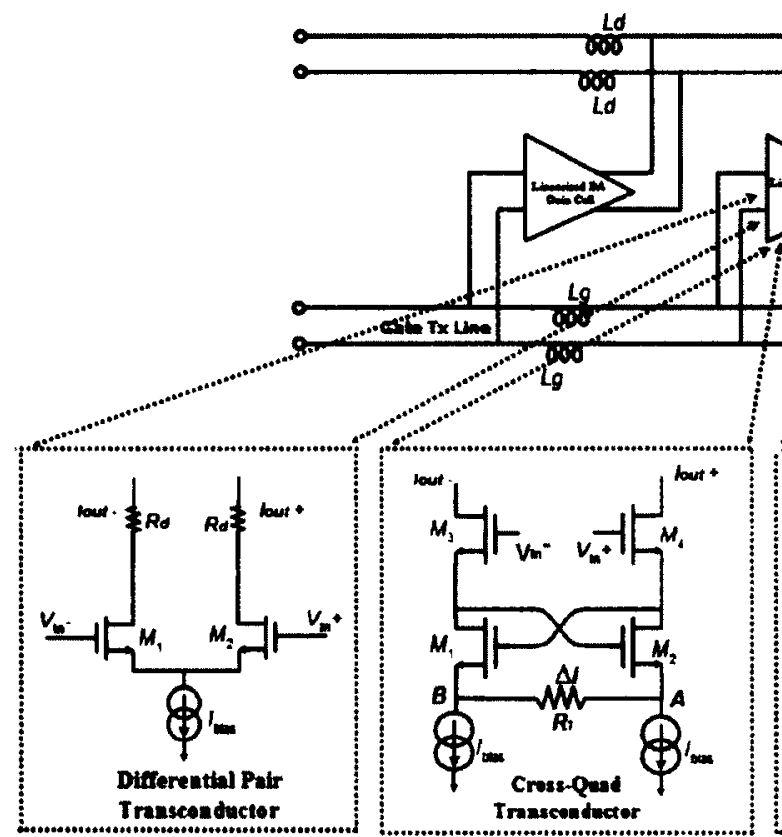

(a)

(b)

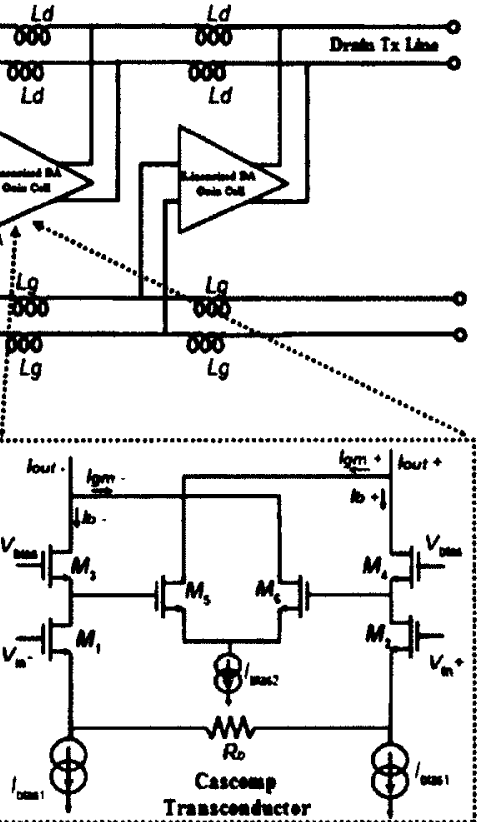

(c)

Figure 3.7: (a) CMOS source degeneration transconductor (b) Caprio's cross-quad transconductor (c) Quinn's cascomp transconductor as a gain cell application for highfrequency amplifiers linearity enhancement.

linearization circuitry can be added to the differential pair amplifier in order to compensate for the transconductance nonlinearity. This allows the nonlinear amplifier to be used to amplify signals utilizing spectrally efficient linear modulation techniques without causing interference. Many techniques have been developed to improve the linearity of the basic differential pair transconductor such as source degeneration, Caprio's cross-quad [21] and Quinn's cascomp transconductor [74] as shown in Figure 3.7 (a), (b) and (c) respectively. However, their transconductance second-derivative gm" nulling is not sufficiently wide and their gm flatness is limited ( [64], [75]). Other published linearized BJT V-I converters, such as the proposed linearized transconductor in [76], does not linearize the total transconductor drain current instead only linearizing the inner translinear loop current. Other published linearized transconductors lack on-chip tuning [56].

A three-stage bidirectional distributed amplifier with a differential pair transconductor as a gain stage is shown Figure 3.7 (a) coupling the drain and gate transmission-lines. 
The linearity characteristic of the differential pair is determined by the non ideality of the transistors. The transconductance $g_{m}$ of the MOS device varies with the input voltage level. This causes distortions in the drain current which results in a distorted output voltage at the load of the distributed amplifier.

The transfer characteristic function of the differential pair transconductor can can be expressed as ([16], [60])

n

$$
\Delta V_{\text {out }}=-\frac{1}{2} \mu_{n} C_{o x} \frac{W}{L} R_{d} \Delta V_{\text {in }} \sqrt{\frac{4 I_{\text {bias }}}{\mu_{n} C_{\text {ox }} \frac{W}{L}}-\Delta V_{\text {in }}^{2}}
$$

The small-signal gain $A_{v}$ of the differential pair transconductor can be obtained through differentiating the transfer characteristic function in 3.3.1

$$
A_{v}=\frac{\delta V_{\text {out }}}{\delta V_{\text {in }}}=-R_{d} \sqrt{\mu_{n} C_{o x} \frac{W}{L} I_{\text {bias }}}=-R_{d} G_{m}
$$

where $G_{m}=g_{m 1}=g_{m 2}$ is the overall transconductance of the differential pair transconductor.

The linearity characteristic of the differential pair transconductor is determined by the non-ideality of the transistors. To observe the linearity characteristics of the differential amplifier transconductor, its transfer function in Equation 3.3.1 can be modeled by a third order polynomial Taylor series

$$
V_{\text {out }}=a_{1} V_{\text {in }}+a_{2} V_{\text {in }}^{2}+a_{3} V_{\text {in }}^{3}
$$

The polynomial coefficients can be simplified by partial derivatives

$$
\begin{gathered}
a_{1}=\left.\frac{\partial V_{\text {out }}}{\partial V_{\text {in }}}\right|_{\text {vin }=0}=-R d \sqrt{\mu_{n} C_{o x} \frac{W}{L} I_{\text {bias }}} \\
a_{2}=\left.\frac{\partial^{2} V_{\text {out }}}{\partial V_{\text {in }}^{2}}\right|_{\text {vin }=0}=0 \\
a_{3}=\left.\frac{\partial^{3} V_{\text {out }}}{\partial V_{\text {in }}^{3}}\right|_{\text {vin }=0}=\frac{3}{4} R_{d} \frac{\left(\mu_{n} C_{o x} \frac{W}{L}\right)^{\frac{3}{2}}}{\sqrt{I_{\text {bias }}}}
\end{gathered}
$$


Since the differential pair transconductor generates no $2^{\text {nd }}$ order nonlinearity. The denominator of $a_{3}$ in Equation 3.3.6 consists of the square root of bias current $I_{\text {bias }}$. Consequently, increasing the bias current $I_{b i a s}$ lowers the coefficient $a_{3}$ and improves the linearity of the the differential pair transconductor and hence overall distributed amplifier linearity. However, improving the linearity by increasing bias current $I_{\text {bias }}$ has its limits. A higher $I_{\text {bias }}$ increase power consumption.

The overall linearity of the DA can be improved as well by using Caprio's cross-quad transconductor as a gain stage shown in Figure 3.7 (b). Caprio proposed a precision differential voltage-current converter linearization technique [21]. The cross-quad linearization technique shown in Figure 3.7 (b) uses a unity gain positive feedback in cross quad to synthesize a virtual ground across a passive element $R_{1}$. The positive feedback Caprio's cross-quad linearization technique is independent of $g_{m}$ as in conventional degeneration method. Assuming perfect matching and ignoring body effects, the gate-source voltages of $M_{3}$ and $M_{2}$ are compensated by gate-source voltages of $M_{4}$ and $M_{1}$ shown in Figure 3.7 (b).

$$
V_{A}=\left(V_{i n}^{+} / 2\right)-V_{G S 2}-V_{G S 3}
$$

where $V_{G S 2}$ is the gate-source voltage of transistor $M_{2}$ and $V_{G S 3}$ is the gate-source voltage of transistor $M_{3}$ of Caprio's transconductor shown in Figure 3.7 (b).

$$
V_{B}=\left(V_{i n}^{+} / 2\right)-V_{G S 1}-V_{G S 4}
$$

where $V_{G S 1}$ is the gate-source voltage of transistor $M_{1}$ and $V_{G S 4}$ is the gate-source voltage of transistor $M_{4}$ of Caprio's transconductor shown in Figure 3.7 (b).

Therefore, the overall Caprio's transconductor drain current is linearly proportional to the input voltage

$$
\Delta i=\left(\frac{V_{A}-V_{B}}{R_{1}}\right)=\frac{V_{i n}}{R_{1}}
$$

where $\Delta i$ is the overall Caprio's transconductor drain current and $V_{i n}$ is the input voltage.

In Caprio's cross-quad, all four transistors $M_{1}$ to $M_{4}$ have the same (W/L) transistor size and are perfectly matched. The gate-source voltages for $M_{1}$ to $M_{3}$ are the same as 
the gate-source voltages for $M_{2}$ to $M_{4}$ since the current flowing through the left branch of the cross quad is the common to $M_{1}$ to $M_{3}$, while the current flowing through the right branch is common to $M_{2}$ to $M_{4}$.

The overall linearity of the DA can be improved by using the cascomp (cascode compensator) transconductor as shown in Figure 3.7 (c). A three-stage bidirectional DA with a cascomp transconductor as a gain stage is shown in Figure 3.7 (c). The Cascomp transconductor circuit as shown in Figure 3.7 (c) for feedforward linearization was proposed by Quinn [74]. Ignoring body effects, the inner differential pair $M_{5}$ and $M_{6}$ subtracts the difference in gate-source voltages of $M_{1}$ and $M_{2}$ to linearize the transconductance [74] as shown in Figure 3.7 (c).

$$
V_{R b}=V_{\text {in }}+V_{G S 1}-V_{G S 2}=V_{\text {in }}-\Delta V_{G S}=V_{R b} \Delta i
$$

where $V_{G S 1}$ is the gate-source voltage of transistor $M_{1}$ and $V_{G S 2}$ is the gate-source voltage of transistor $M_{2}$ of Cascomp transconductor shown in Figure 3.7 (c).

$$
V_{A B}=\left(V_{B I A S}-V_{G S 3}\right)-\left(V_{B I A S}-V_{G S 4}\right)=V_{G S 1}-V_{G S 2}
$$

where $V_{G S 3}$ is the gate-source voltage of transistor $M_{3}$ and $V_{G S 4}$ is the gate-source voltage of transistor $M_{4}$ of Cascomp transconductor shown in Figure 3.7 (c).

Once $\Delta V_{G S}$ is applied to the differential pair of $M_{5}$ and $M_{6}$, it drives the inverse drain currents for $M_{3}$ and $M_{4}$. Therefore, if

$$
\begin{gathered}
g_{m 5}=g_{m 6}=2 / R b \\
i_{1}-i_{2}=2 \frac{V_{\text {in }}}{R_{b}}
\end{gathered}
$$

Therefore, the overall Cascomp transconductor drain current is linearly proportional to the input voltage. 


\subsection{Chapter Summary}

In this chapter, analysis of the distributed amplification principles were presented in this chapter. Different linearized transconductors to compensate for the nonlinearity such as cascomp and caprio's transconductors were also presented. 


\section{Chapter 4}

\section{Distributed RF Linearization Circuit Applications}

\subsection{Introduction}

The interest in high-level integration and highly linear multi-functional broadband subsystems motivates the development of linearized distributed circuit applications. In this chapter, the application of RF linearization to distributed circuit functions such as fullyintegrated CMOS linearized distributed active power splitter (unbalanced input, balanced output) and a linearized CMOS distributed matrix amplifier are presented. Also the linearized CMOS distributed paraphase amplifier employing derivative superposition linearization is presented as well.

\subsection{Linearized CMOS Distributed Active Power Split- ter}

The design of a fully-integrated CMOS distributed active power splitter (unbalanced input, balanced output) incorporating multiple-gated transistor linearization that allows for broadband distortion reduction is presented in this section. Broadband power splitters are common elements found in many communication systems. Power splitters are found in phased antenna arrays ( [77], [78]). The Wilkinson power splitter was invented around 1960 by an engineer named Ernest Wilkinson ( [79], [80]). It splits an input signal into two equal phase output signals however the passive Wilkinson power splitter commonly used 

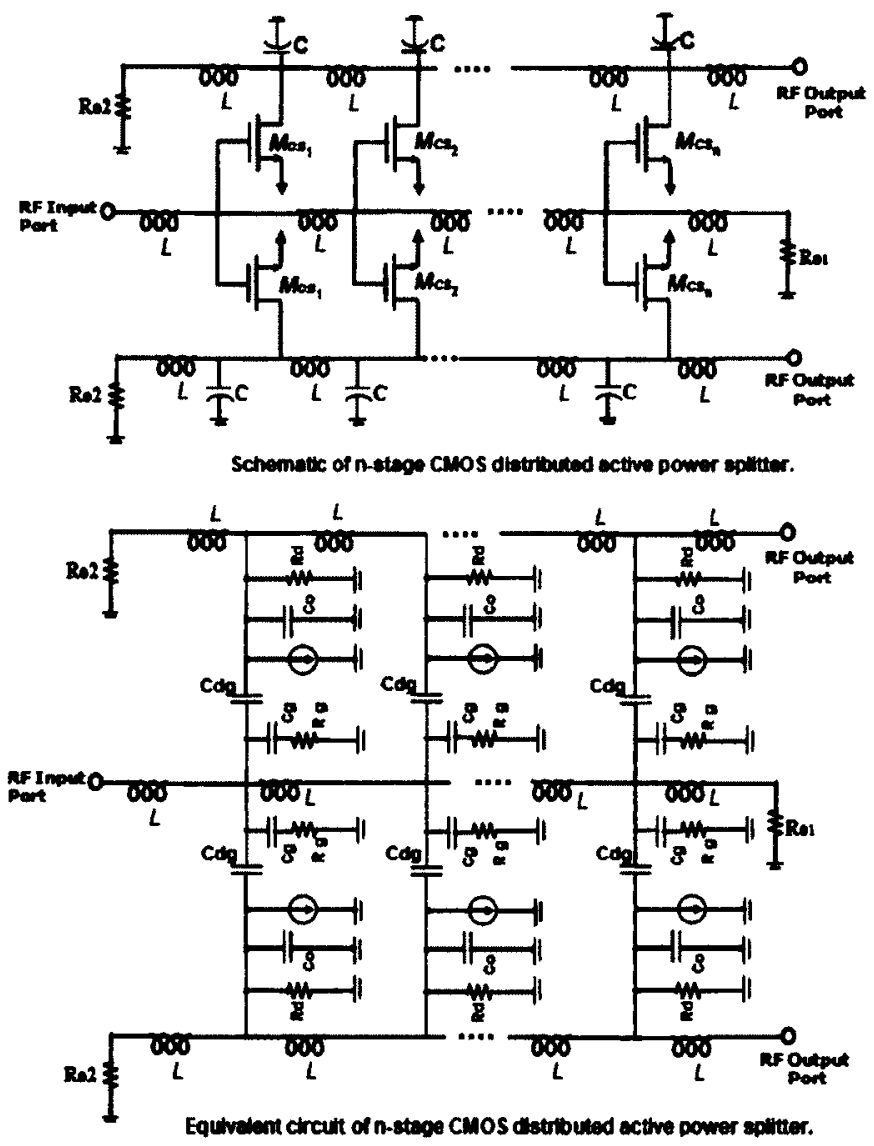

Figure 4.1: Block diagram illustration of CMOS distributed active power splitter. 
has an inherent $3 \mathrm{~dB}$ loss. The active power splitter provide an attractive alternative to the conventional Wilkinson or other planar power splitters in terms of gain, isolation and size. Hence, am active ultra-wideband circuit that can perform the same splitting function is presented.

The CMOS linearized distributed power splitter provides two outputs, with equal phase over a wide microwave band with minimal phase and amplitude imbalance. Compared to passive power splitter circuits, the linearized CMOS distributed power splitter's advantage is that it provides gain and allows for broadband distortion cancellation. The proposed CMOS linearized distributed power splitter makes up for the inherent $3 \mathrm{~dB}$ loss of passive power splitters and be used to drive balanced antennas and wideband phase shifters.

The wideband distributed power splitter is based on the concept of the distributed amplifier ( [19], [20]). The distributed amplifier is a classical topology for wideband amplifiers since the distributed amplifier has several attractive characteristics such as flat gain, good input and output matching as well as small size. The distributed amplifier design guidelines given by Beyer [20] can be extended to include linearized CMOS distributed power splitter. Figure 4.1 shows a schematic of an n-stage distributed power splitter and its equivalent circuit. It consists of n-stage distributed FET amplifiers. The balanced output transmission lines both have a distributed common-source amplifier. They share a common unbalanced input transmission line. A simple circuit model for the distributed active power splitter is shown Figure 4.1.

The power gain of the common-source section of the distributed power splitter shown in Figure 4.1 is given by [20]

$$
\begin{gathered}
G_{C S}=\frac{G_{m}^{2} R_{o g} R_{o d}}{4\left[1+\varpi_{/ \varpi_{g}}\right]^{2} 1-\varpi_{/ \varpi_{c}}{ }^{2}} \\
\times \frac{\sinh ^{2}\left[\frac{n}{2}\left(\alpha_{d}-\alpha_{g}\right)\right] e^{-n\left(\alpha_{d}+\alpha_{g}\right)}}{\sinh ^{2}\left[\frac{1}{2}\left(\alpha_{d}-\alpha_{g}\right)\right]}
\end{gathered}
$$

where $G_{m}$ is the transconductance and $\alpha_{d}$ and $\alpha_{g}$ are the attenuation per section and $R_{o d}$ and $R_{o g}$ are the characteristic resistance and $\varpi_{d}$ and $\varpi_{g}$ are of the drain and gate line respectively.

The amplitude imbalance of the distributed active power splitter can be expressed as $[20]$ 


$$
\begin{gathered}
\Delta A(d B)=20 \log \frac{1+\frac{1}{G_{m} R_{d}}}{1+\frac{z_{d \alpha}}{2 R_{d}}}+20 \log \frac{\cosh \left(\frac{n}{2} \frac{\alpha_{d}}{\alpha_{o}}\right)}{\cosh \left(\frac{1}{2} \frac{\alpha_{d}}{\alpha_{o}}\right)} \\
-10 \log \left[\exp \left(n \frac{\alpha_{d}}{\alpha_{o}}\right)\right]
\end{gathered}
$$

$\alpha_{d}$ and $\alpha_{g}$ are the attenuation per section and $G_{m}$ is the transconductance.

The first term results from the gain difference between the two stages while the latter two terms stem from the difference in attenuation on the two output lines.

The phase imbalance of more practical concern is given by [20]

$$
\delta \phi=\arctan \frac{\varpi C_{d}}{\frac{1}{R_{d}}+G_{m}}-\arctan \frac{\varpi C_{d}}{\frac{1}{R_{d}}+\frac{2}{Z_{d \pi}}}
$$

where $G_{m}$ is the transconductance. The critical design parameters are output line impedance level and device $G_{m}$ transconductance. For instance, if output line impedance is dictated by circuit constraints the device can be chosen to minimize the phase imbalance.

\subsubsection{Amplitude and Phase Imbalance of Linearized CMOS Dis- tributed Active Power Splitter}

The inherent broadband characteristics of the distributed amplifier is applied to a linearized CMOS distributed active power splitter design. The balanced output transmission lines both have a distributed common-source amplifier. They are fed by a single input transmission line. The characteristic low pass response of the distributed amplifier then yields two RF outputs from one RF input signal, the amplitudes of both RF output signals are equal and their phase are equal over a wide band ([79], [80]). Figure 4.6 shows the schematic of the proposed linearized CMOS distributed power splitter. A distributed pre-amplifier stage is added which not only supplies gain but serves as an active impedance transformer.

The lumped transmission lines tend to provide a constant input and output resistance over a wide passband. As a result, the phase difference between the balanced output distributed common-source amplifier transmission lines is theoretically zero degrees, independent of the frequency, if the phase velocities of the signals on the three transmission lines are identical. The drain and gate transmission-line inductors have an inductance value of $1.1 \mathrm{nH}$ and the drain transmission-line $\mathrm{m}$-derived inductance is $120 \mathrm{pH}$ with 50 Ohms terminations. The device dimensions $\left[M_{1}, M_{3}\right](\mathrm{W} / \mathrm{L})$ and $\left[M_{c s 1}, M_{c s 2}\right](\mathrm{W} / \mathrm{L})$ have 


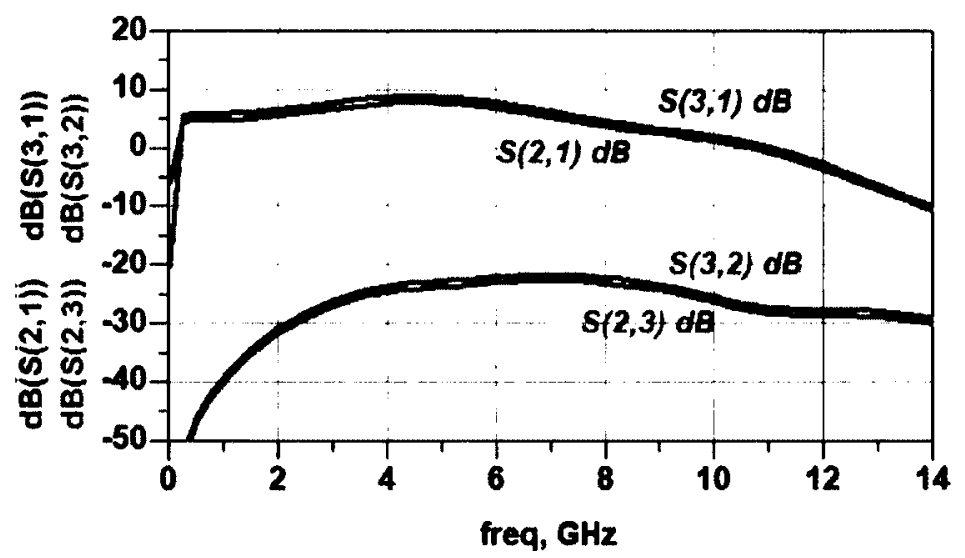

Figure 4.2: Simulated output power S21 common-source stage port and S31 commonsource stage port peaks at $7.8 \mathrm{~dB}$ for the proposed linearized CMOS distributed power splitter.

a width equal to $44 \mu \mathrm{m}$ with all devices having $\mathrm{L}$ minimum channel length of $120 \mathrm{~nm}$. The device dimensions $\left[M_{a}, M_{c}\right](\mathrm{W} / \mathrm{L})$ and $\left[M_{c s a}, M_{c s b}\right](\mathrm{W} / \mathrm{L})$ have a width equal to $22 \mu \mathrm{m}$ with all devices having $\mathrm{L}$ minimum channel length of $120 \mathrm{~nm}$. The power gains of the common-source amplifier on both outputs are almost the same with nearly equal output power as can be seen in Figure 4.2 and as a result, a wideband CMOS distributed power splitter is obtained.

The linearized CMOS distributed power splitter has a $7.8 \mathrm{~dB} S_{21}$ power gain peak as shown in Figure 4.2 and rolls off to a unity gain bandwidth of $10.5 \mathrm{GHz}$. The phase imbalance is less than 5 degrees as shown in Figure 4.5 and the amplitude imbalance is less than $1 \mathrm{~dB}$ over the band as shown in Figure 4.3. The simulated phase S21 and phase S31 for the proposed linearized CMOS distributed power splitter is shown in Figure 4.4.

\subsubsection{CMOS Distributed Active Power Splitter Using Multiple- Gated Transistor Linearization}

Active MOSFET devices can be modeled by nonlinear current and charge sources that depend on the device voltages ( [12], [13]). These nonlinear sources will give rise to distortion when driven with a modulated signal. The real MOSFET device output impedance 


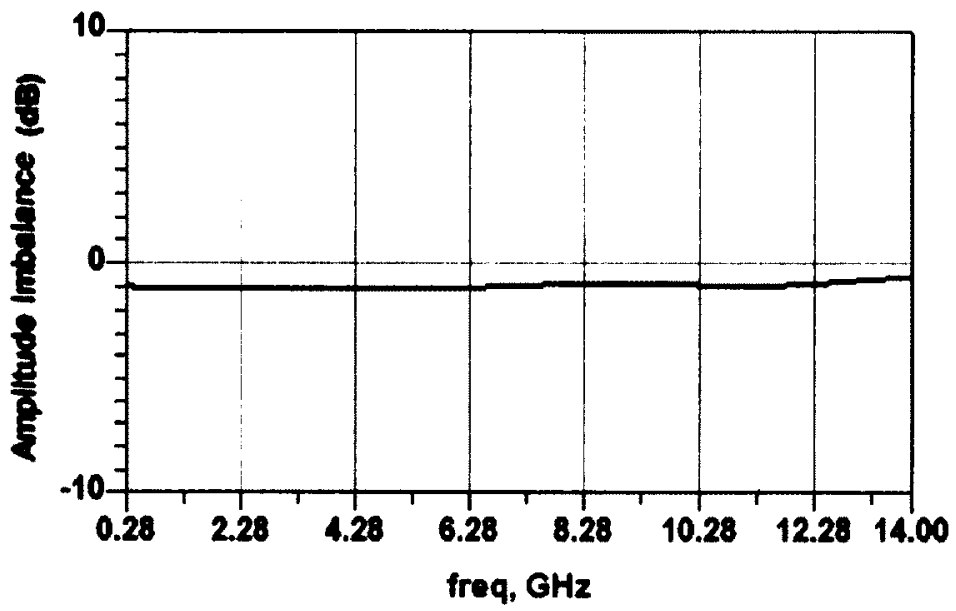

Figure 4.3: Simulated amplitude imbalance for the proposed linearized CMOS distributed power splitter amplitude imbalance.

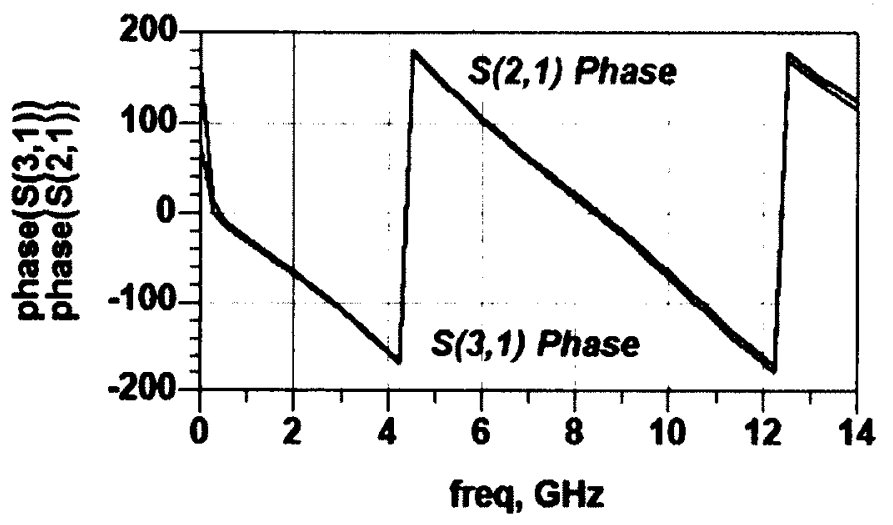

Figure 4.4: Simulated phase S21 and phase S31 for the proposed linearized CMOS distributed power splitter. 


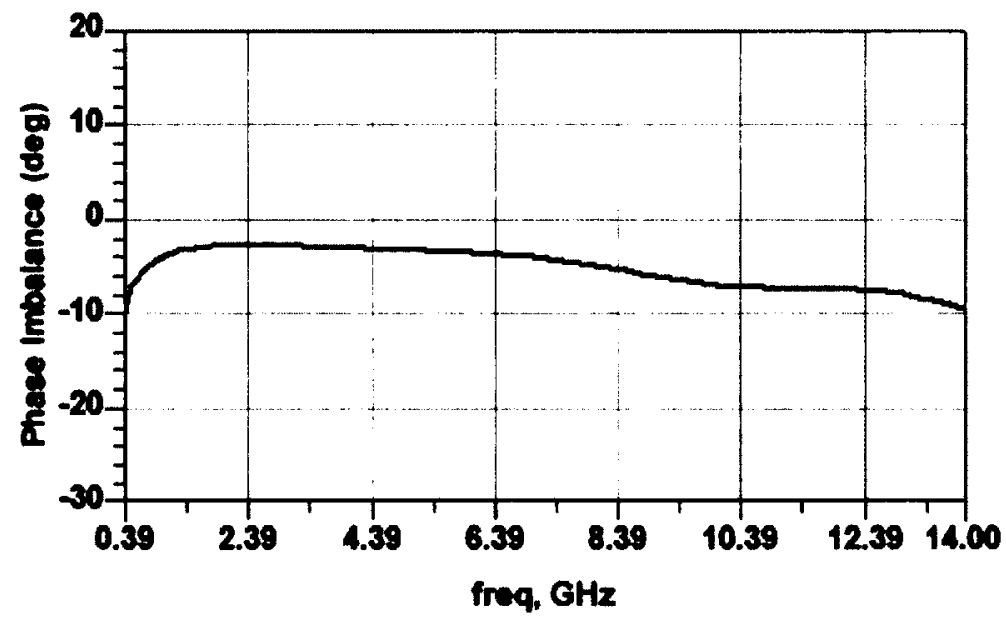

Figure 4.5: Simulated phase imbalance for the proposed linearized CMOS distributed power splitter.

is nonlinear and the mobility $\mu$ is not a constant but a function of the vertical and horizontal electric field. We may bias the active MOSFET device where the device behavior is more exponential. And there is also an internal feedback so when the input signal driven into the amplifier is increased, the output is also increased until a point where distortion products can no longer be ignored ( [12], [14]). No transistor is perfectly linear since the inherent nonlinearity of the diode junctions that comprise many of the active devices found in most amplifiers. The harmonics of the output signal are generated by nonlinearities of the MOSFET devices. The major three nonlinear elements of the MOSFET devices are nonlinear transconductance $g_{m}$, the device drain capacitance $C_{d}$ and gate capacitance $C_{g s}$ ( [12], [15]). The real MOSFET devices generate higher order distortion ( [16], [15]).

There are many linearization techniques to increase the linearity of the amplifier such as feedforward or multi-tanh ( [81], [82], [83], [84]) However they are better suited for differential circuits and hence consume more power and silicon area than linearization techniques applied to single-ended circuits. A simple linearization technique using multiplegated common source transistors is used to linearize the single-ended CMOS distributed power splitter, where gate drive width and gate drive $\left(V_{g s}-V_{t h}\right)$ of each transistor are chosen 


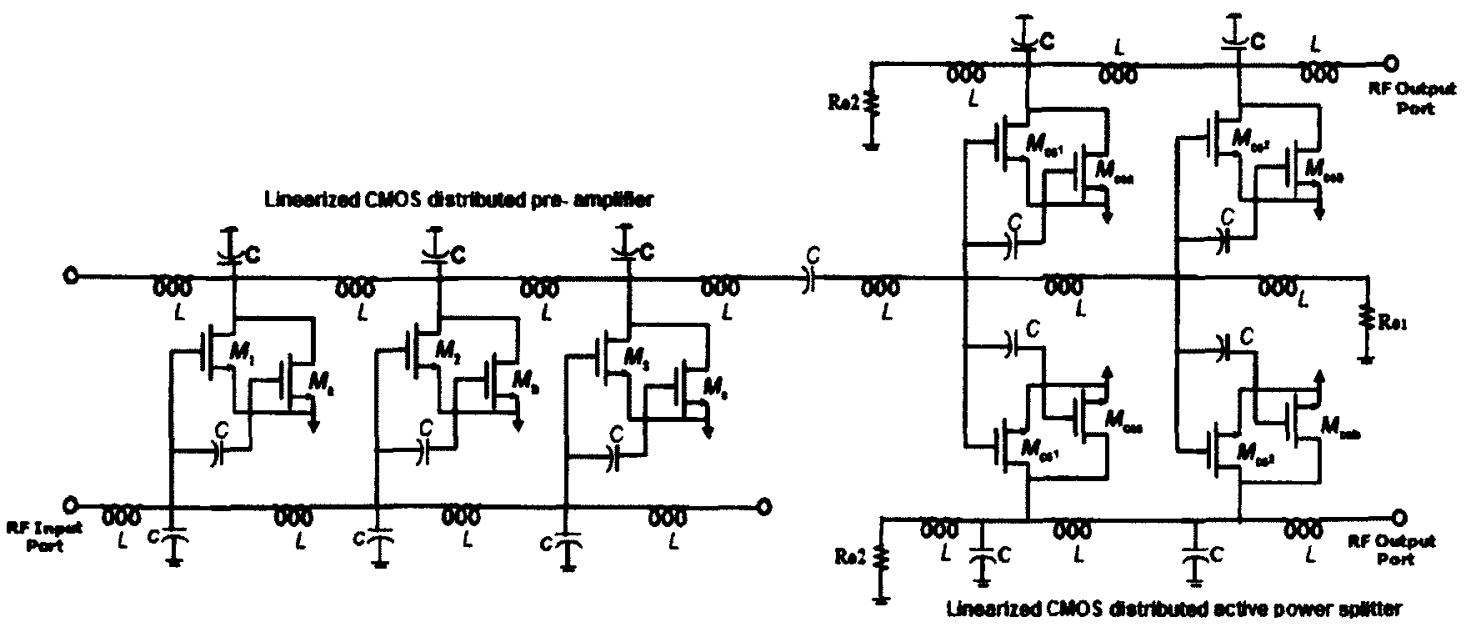

Figure 4.6: Schematic topology of the proposed fully-integrated linearized CMOS distributed active power splitter.

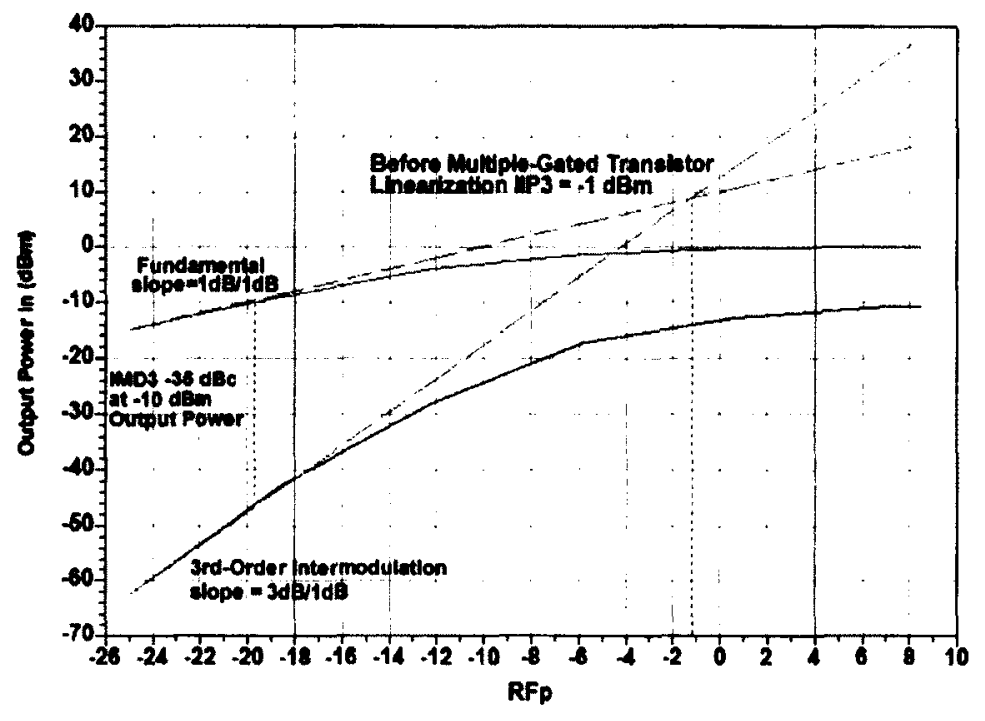

Figure 4.7: ADS simulation of IIP3 before CMOS distributed active power splitter multiplegated transistor linearization. 
to compensate for the nonlinear characteristic of the main transistor. Using Taylor series expansion, the drain current of a common source FET can be expressed as ( [85], [86], [87])

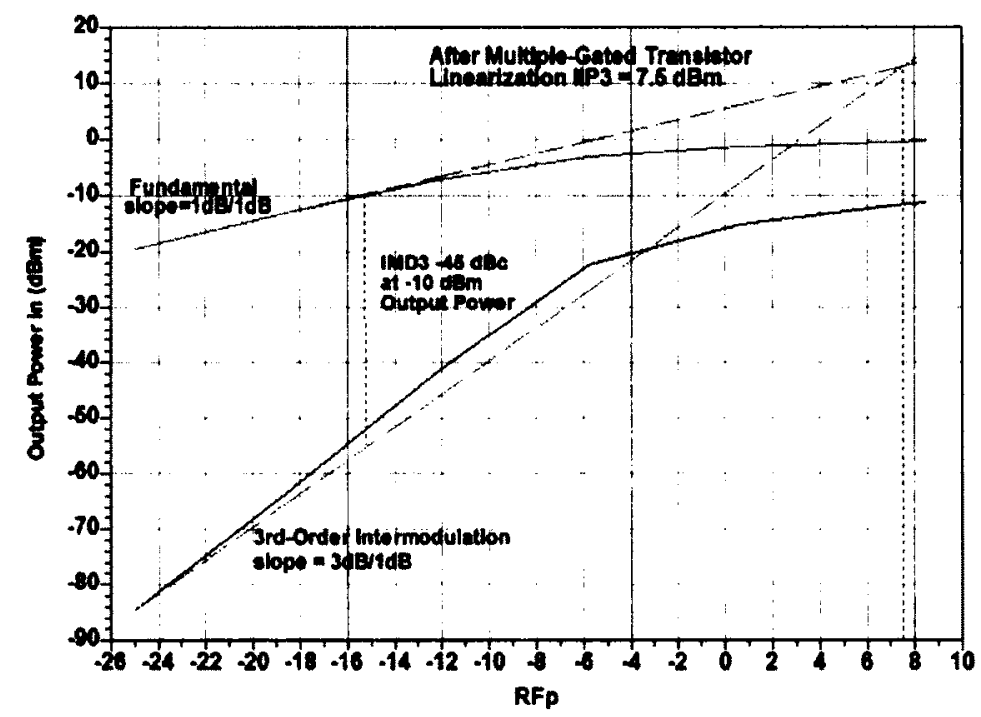

Figure 4.8: ADS simulation of IIP3 after CMOS distributed active power splitter multiplegated transistor linearization with an $8.5 \mathrm{~dB}$ IIP3 improvement and a $10 \mathrm{dBc}$ IMD3 improvement at output power of $-10 \mathrm{dBm}$.

$$
i_{d s}=I_{d c}+g_{m} v_{g s}+\frac{g_{m^{\prime}}}{2} v_{g s^{2}}+\frac{g_{m^{\prime \prime}}}{6} v_{g s^{3}}+\ldots
$$

where $g_{m^{\prime}}$ and $g_{m^{n}}$ are the first and second transconductance derivatives, respectively, with respect to the gate to source voltage. The negative $g_{m}$ of the main transistor can be canceled by the positive $g_{m^{n}}$ of the secondary transistor which is biased at a smaller gate drive as can be depicted in Figure 4.9. The amount $g_{m}$ compensation can be chosen by adjusting the width of secondary transistor. The compensated flat region of the overall transfer characteristic curve of both main and secondary transistor can be extended farther with proper bias voltage and transistor size to synthesize an overall transfer function with reduced nonlinearities ( [79], [80]).

In this section, the multiple-gated transistor linearization technique is applied to the single-ended linearized CMOS distributed active power splitter design. The tuning of the 


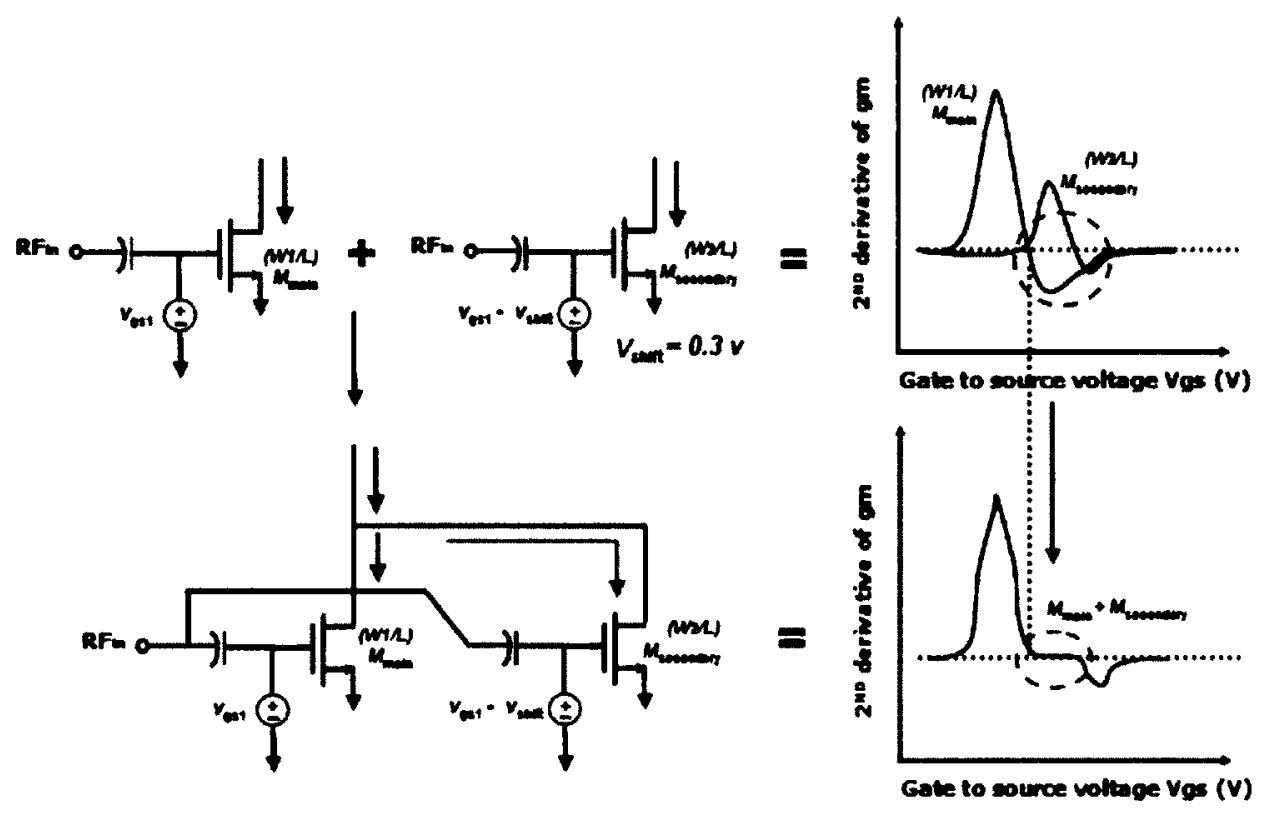

Figure 4.9: Schematic illustration of multi-gated transistor linearization technique. The gate bias and the transistor size of the secondary transistor is chosen such that the negative $2^{\text {nd }}$ derivative of $g_{m}$ peak of main transistor is canceled by the positive one of secondary transistor. 
power series nonlinear coefficient, transconductance $g_{m^{n}}$, and its effect on IIP3 is shown in Figure 4.7 and Figure 4.8. As can be seen in Figure 4.7 and Figure 4.8 with proper bias voltage and transistor sizes the third-order nonlinearity can be canceled. Both IIP3 is improved by $8.5 \mathrm{~dB}$ and the third order intermodulation (IMD3) is improved by $10 \mathrm{dBc}$ improvement, at output power of $-10 \mathrm{dBm}$, can be seen in Figure 4.8.

A common way to characterize the non-linear amplitude distortion of an amplifier under a two tone input is using the third order intercept point. This is the operating point where the power in the fundamental and the power in the third order intermodulation product are the same. In the linear region, third-order products increase by $3 \mathrm{~dB}$ for every $1 \mathrm{~dB}$ increase of input power, while output power increases by $1 \mathrm{~dB}$. The output power where the two would intersect is the third order intercept point, and is a good indicator of the linearity of an amplifier

The design of a fully-integrated linearized CMOS distributed active power splitter that allows for broadband distortion reduction is presented in this section. Simulation results has yielded a peak $S_{21}$ and $S_{31}$ peak power gain of $7.8 \mathrm{~dB}$ and then rolls off to a unity gain bandwidth of $10.5 \mathrm{GHz}$ with less than 5 degrees phase imbalance and amplitude imbalance of less than $0.9 \mathrm{~dB}$ over the band. The simulation results show an $8.5 \mathrm{~dB}$ IIP3 improvement and a $10 \mathrm{dBc}$ improvement at output power of $-10 \mathrm{dBm}$ and it has broadband signal transmission gain that compensates loss. The proposed fully-integrated design eliminates the need for off-chip discrete components and is suitable for ultra-wideband wireless transceiver applications.

\subsection{Linearized CMOS Distributed Matrix Amplifier Architecture}

The interest in high-level integration and multi-functional subsystems motivates the development of broadband amplifiers. Distributed amplifiers have become the leading candidate for ultra-wideband amplification at microwave frequencies. A major design challenge for ultra-wideband amplifiers is the stringent linearity requirement over a wide bandwidth 
range, due to large numbers of in-band interferences in ultra-wideband wireless communication systems. Distributed Matrix amplifiers find many applications in broadband microwave applications and wireless communications ([88], [89], [90], [91]). A fully-integrated CMOS linearized interleaved distributed $2 \times 3$ matrix amplifier employing active post distortion and optimum gate bias linearization technique is presented in this section. The proposed CMOS linearized interleaved distributed $2 \times 3$ matrix amplifier incorporates a third-order intermodulation distortion IM3 suppression technique with simple circuit implementation and negligible extra power consumption that is well suited for integrated wireless broadband transceivers.

To apply the additive and multiplicative gain mechanism simultaneously for optimum amplifier performance, a matrix architecture for distributed amplifiers has been employed for circuit implementations ( [59], [92], [93]). Distributed matrix amplifier is a type of amplifier that combines the additive and the multiplicative principles in one and the same module. It is possible to achieve both additive and multiplicative amplification by having two distributed amplifiers in a way that resembles the distribution of elements in an array therefore the name matrix amplifier as shown in Figure 4.10. This two-tier matrix amplifier, which consists of a rectangular array of CMOS active devices, is composed of three periodically loaded artificial transmission lines: input line, central line, and output line. The input signal is amplified from one tier to the next before the final output signal leaves the uppermost drain-line output. This means that both multiplicative gain (one transistor output feeding the next's input) and additive gain (the summing of transistor outputs on the same drain-line) are provided within the single module. The structure is quite compact and allows circuits with higher gain per unit area as compared with conventional distributed amplifiers ( [67], [65], [21], [74], [94]). Figure 4.10 shows a schematic of a interleaved distributed $2 \times \mathrm{n}$ matrix amplifier and its equivalent circuit.

\subsubsection{CMOS Distributed 2 x 3 Matrix Amplifier with Interleaved Distributed Loading Technique}

In conventional distributed matrix amplifier, heavy loading on the central transmission line limits the bandwidth performance. Introducing an interleaved distributed loading technique would reduce the loading on the central artificial transmission line thus extending 


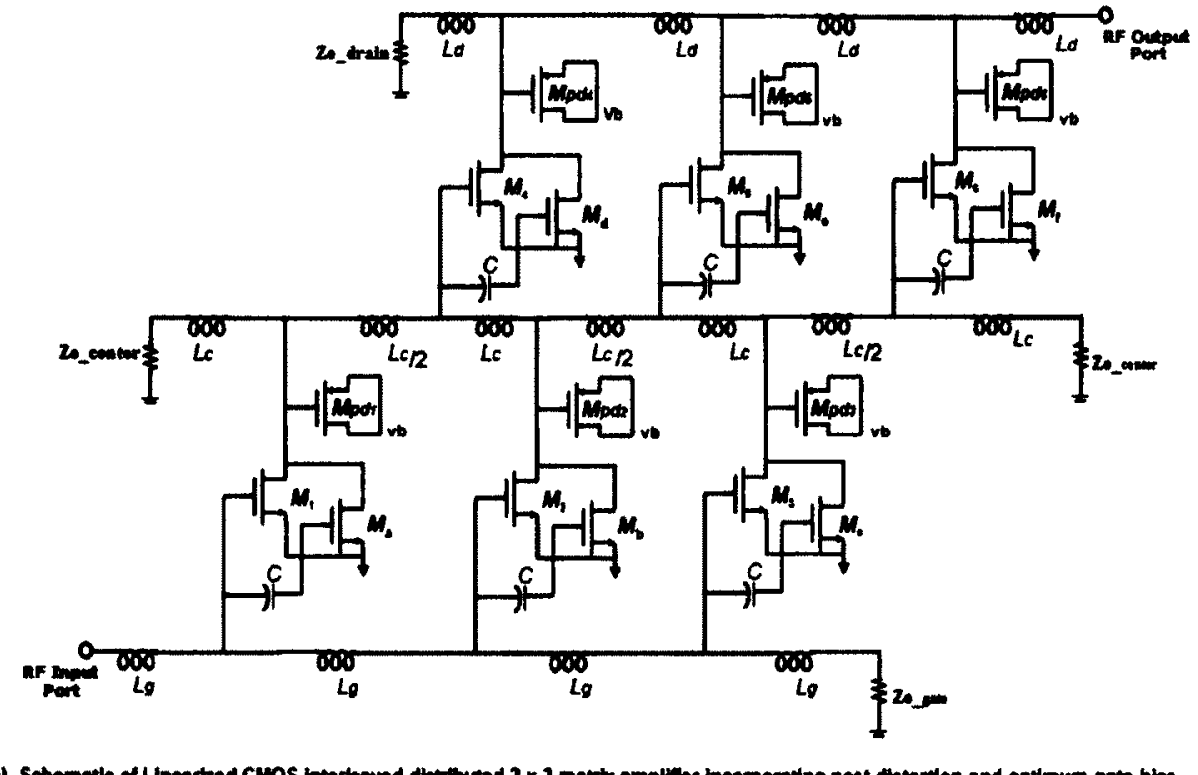

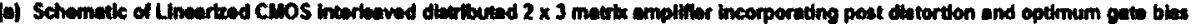
Inecribiton mechnique.

Figure 4.10: Schematic topology of the proposed fully-integrated linearized CMOS interleaved distributed $2 \times 3$ matrix amplifier employing active post distortion and optimum gate bias linearization technique. 
the bandwidth. The output loading of the first tier and the input loading of the second tier are separated by an equivalent inductance $L c$ in the proposed circuit topology as shown in Figure 4.10. With the distributed loading technique, a significant performance improvement in terms of the bandwidth of the matrix amplifier can be achieved.

The central transmission line frequency $f_{c}$ and characteristic impedance $Z_{o c}$ for a conventional non-interleaved distributed matrix amplifier can be defined as [76]

$$
\begin{gathered}
f_{c, \text { center }}=\frac{1}{\pi \sqrt{L_{c}\left(C_{d 2}+C_{g 3}\right)}} \\
Z_{o c}=\frac{C_{g 1}}{C_{d 2}+C_{g 3}} Z_{o}
\end{gathered}
$$

where $C_{d}$ is the drain capacitance and $C_{g}$ is the gate capacitance. The transmission line central frequency $f_{c}$ and characteristic impedance $Z_{o c}$ for the interleaved distributed matrix amplifier with distributed loading technique can be defined as [17]

$$
\begin{gathered}
f_{c, \text { center }}=\frac{1}{\pi \sqrt{L_{c} C_{d 2}}}=\frac{1}{\pi \sqrt{L_{c} C_{g 3}}} \\
Z_{o c}=\frac{C_{g 1}}{C_{d 2}} Z_{o}=\frac{C_{g 1}}{C_{g 3}} Z_{o}
\end{gathered}
$$

where $C_{d}$ is the artificial transmission line drain capacitance and $C_{g}$ is the gate capacitance. The conventional non-interleaved central artificial transmission lines of the matrix amplifier suffer from both gate and drain losses. The interleaved distributed matrix amplifier with distributed loading technique has a central transmission line frequency $f_{c}$ equation with less capacitance and thus exhibit higher operational bandwidth, however it trades of with the small-signal gain of the amplifier.

The linearized CMOS interleaved distributed $2 \times 3$ matrix amplifier with distributed loading technique exhibit a $7.1 \mathrm{~dB}$ small signal $S_{21}$ power gain peak as shown in Figure 4.11 and rolls off to a unity gain bandwidth of $16 \mathrm{GHz}$ with less than $-10 \mathrm{~dB}$ return loss and isolation $S_{12}$ of less than $-45 \mathrm{~dB}$ over the band. 


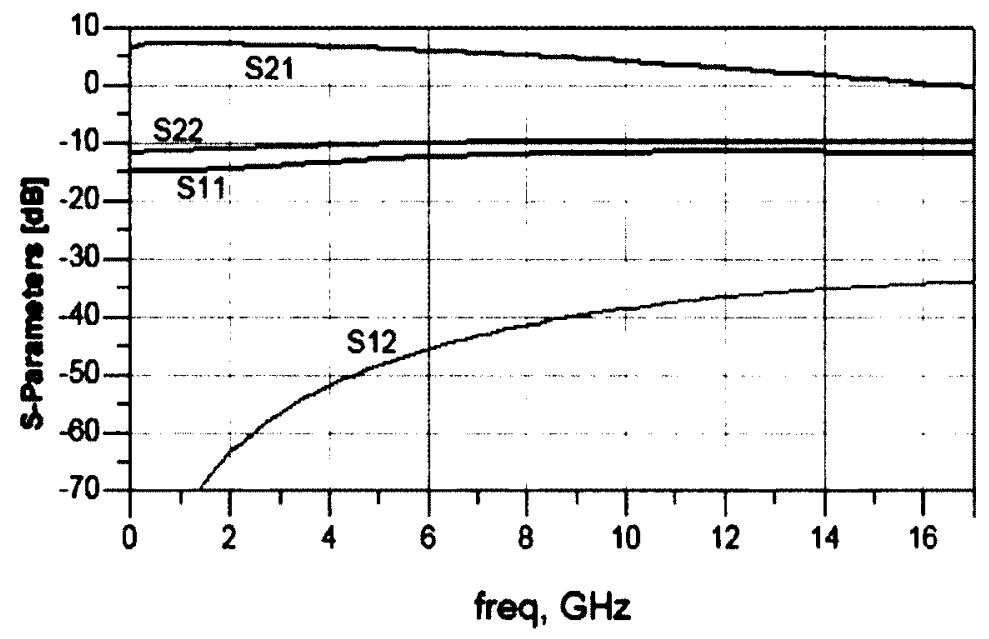

Figure 4.11: Simulated output power S21 peaks at $7.1 \mathrm{~dB}$ and S-parameters for the proposed linearized CMOS interleaved distributed 2 x 3 matrix amplifier.

\subsubsection{Proposed CMOS Interleaved Distributed 2 x 3 Matrix Am- plifier with Post Distortion and Gate Optimum Bias Lin- earization Technique}

We may bias the active MOSFET device where the device behavior is more exponential. The nonlinear MOSFET device sources will give rise to distortion when driven with a modulated signal. When the input signal driven into the amplifier is increased, the output is also increased until a point where distortion products can no longer be ignored ([12], [14]). No transistor is perfectly linear since the inherent nonlinearity of the diode junctions that comprise many of the active devices found in most amplifiers. The harmonics of the output signal are generated by nonlinearities of the MOSFET devices. The major three nonlinear elements of the MOSFET devices are nonlinear transconductance $g_{m}$, the device drain capacitance $C_{d}$ and gate capacitance $C_{g s}$ [15]. The real MOSFET devices generate higher order distortion ( [16], [15]).

In this section, an interleaved distributed matrix amplifier with active post distortion and optimum gate bias distortion cancellation capability which is highly suitable for monolithic integration is presented [95]. The principle of active post distortion is to eliminate the 
considerable contribution to the overall amplifier distortion originating from the transconductance $g_{m}$ nonlinearity. By introducing a linearizer after the input gain stage amplifier, the voltage of the MOS varactor is adjusted to synthesize a transfer function with reduced nonlinearities. The active post distortion linearizer compensates for the transconductance nonlinearities of the input gain stage amplifier producing a linear overall performance and the third-order nonlinearity is canceled as can be seen from Figure 4.12 to Figure 4.19.

The drain and gate transmission-line inductors have an inductance value of $1.3 \mathrm{nH}$ and the drain transmission-line $\mathrm{m}$-derived inductance is $100 \mathrm{pH}$ with $50 \mathrm{Ohms}$ terminations. The device dimensions $\left[M_{1}, M_{6}\right](\mathrm{W} / \mathrm{L})$ have a width equal to $48 \mu \mathrm{m}$ with all devices having $\mathrm{L}$ minimum channel length of $120 \mathrm{~nm}$. The device dimensions $\left[M_{a}, M_{f}\right](\mathrm{W} / \mathrm{L})$ have a width equal to $24 \mu \mathrm{m}$ with all devices having $\mathrm{L}$ minimum channel length of $120 \mathrm{~nm}$. The device dimensions $M_{p d}(\mathrm{~W} / \mathrm{L})$ have a width equal to $40 \mu \mathrm{m}$ with minimum channel length $\mathrm{L}$ of $120 \mathrm{~nm}$.

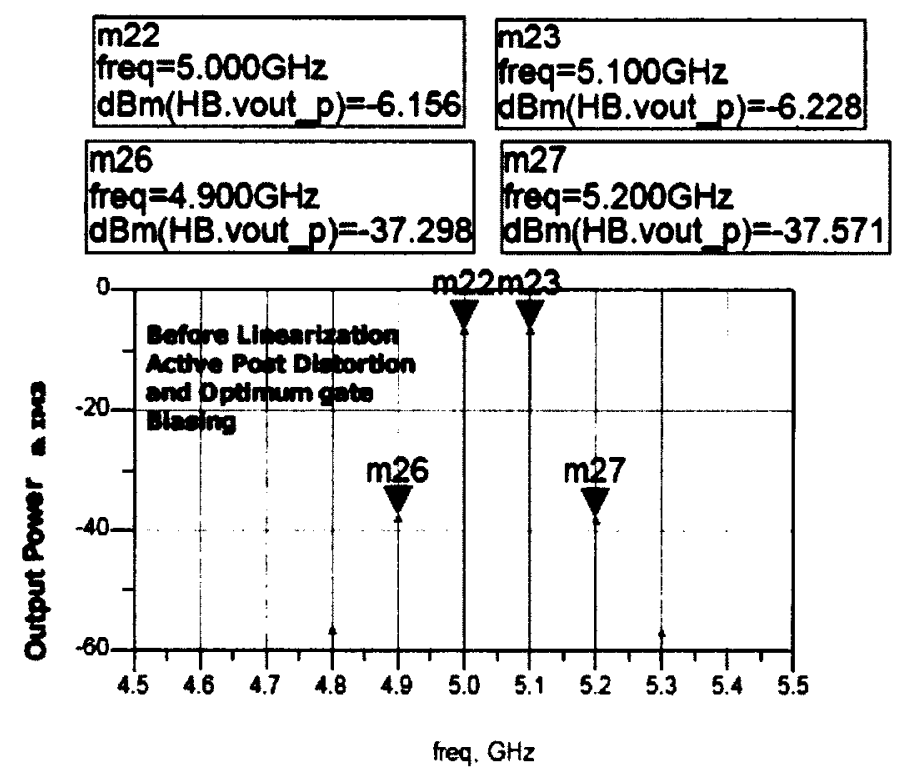

Figure 4.12: Simulated Two-Tone before linearization at $5 \mathrm{GHz}$.

ADS Simulation of IIP3 before CMOS distributed $2 \times 3$ matrix amplifier active post distortion and optimum gate bias linearization is shown in Figure 4.14. Simulation of IIP3 


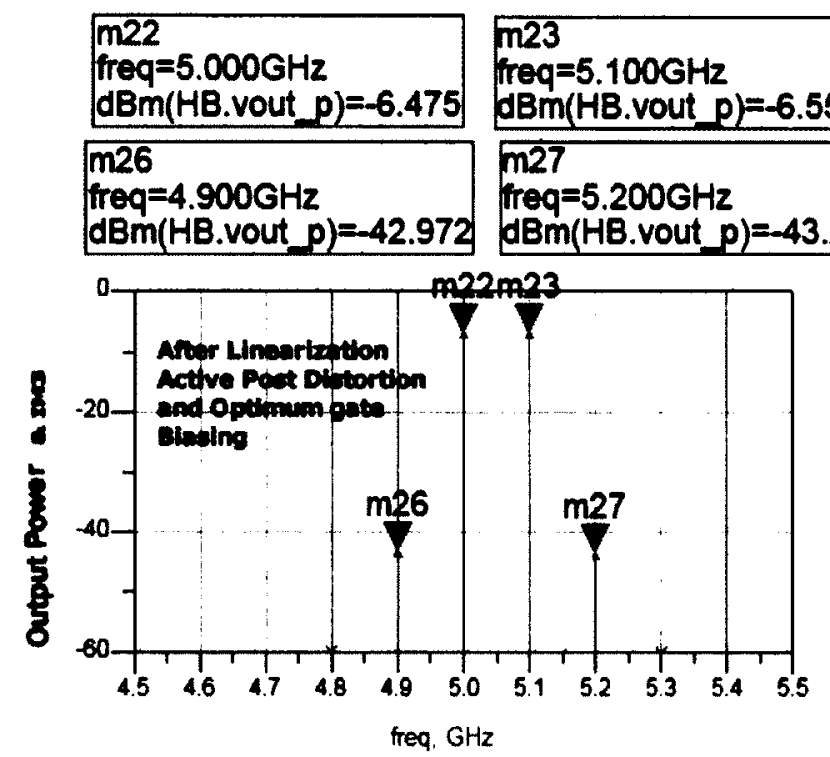

Figure 4.13: Simulated Two-Tone after linearization at $5 \mathrm{GHz}$.

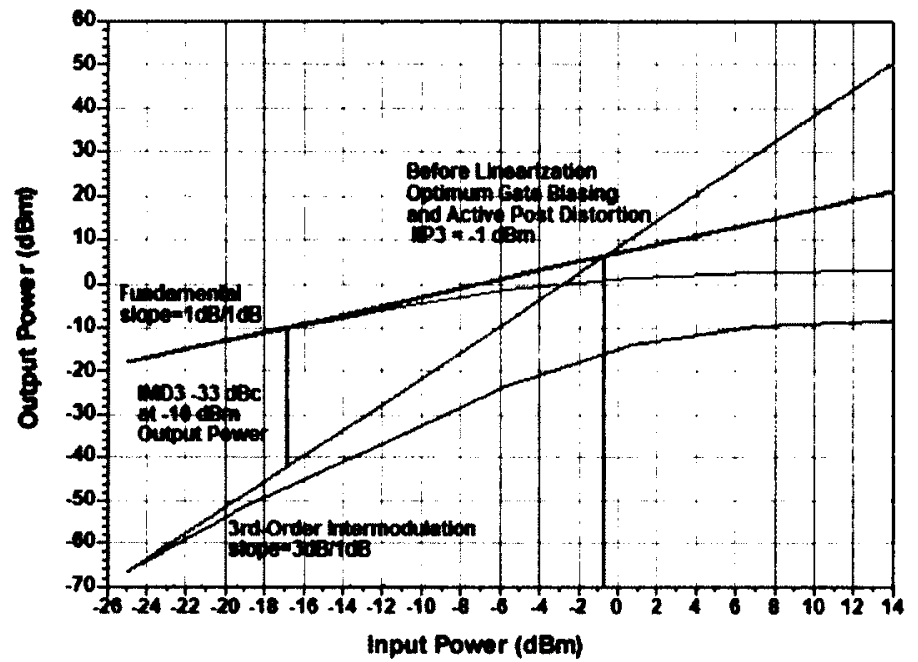

Figure 4.14: ADS Simulation of IIP3 before CMOS distributed $2 \times 3$ matrix amplifier active post distortion and optimum gate bias linearization. 


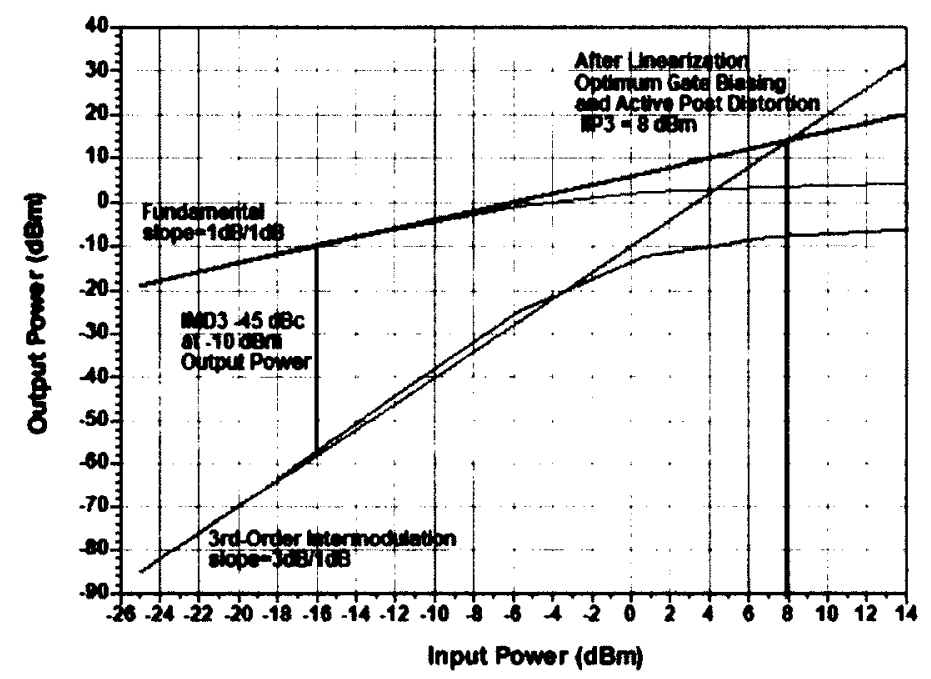

Figure 4.15: ADS Simulation of IIP3 after CMOS distributed $2 \times 3$ matrix amplifier active post distortion and optimum gate bias linearization with a $9 \mathrm{~dB}$ and a $18 \mathrm{dBc}$ improvement at output power of $-10 \mathrm{dBm}$.

after CMOS distributed $2 \times 3$ matrix amplifier active post distortion and optimum gate bias linearization with a $9 \mathrm{~dB}$ and a $18 \mathrm{dBc}$ improvement at output power of $-10 \mathrm{dBm}$ is shown in Figure 4.15.

A common way to characterize the non-linear amplitude distortion of an amplifier is with the $1 \mathrm{~dB}$ compression point. This is the point at which the amplifier gain drops $1 \mathrm{~dB}$ below the linear gain. Simulated 1-dB gain compression point before linearization for the proposed linearized CMOS distributed $2 \times 3$ matrix amplifier is shown in Figure 4.16. Simulated 1-dB gain compression point after linearization for the proposed linearized CMOS interleaved distributed $2 \times 3$ matrix amplifier in Figure 4.17 .

Simulated Two-Tone before active post distortion linearization only at $5 \mathrm{GHz}$ for the proposed linearized CMOS interleaved distributed $2 \times 3$ matrix amplifier is shown in Figure 4.18. Simulated Two-Tone after active post distortion linearization only at $5 \mathrm{GHz}$ for the proposed linearized CMOS interleaved distributed $2 \times 3$ matrix amplifier in Figure 4.19. The concept of optimum gate bias linearization technique is that the negative third-order 


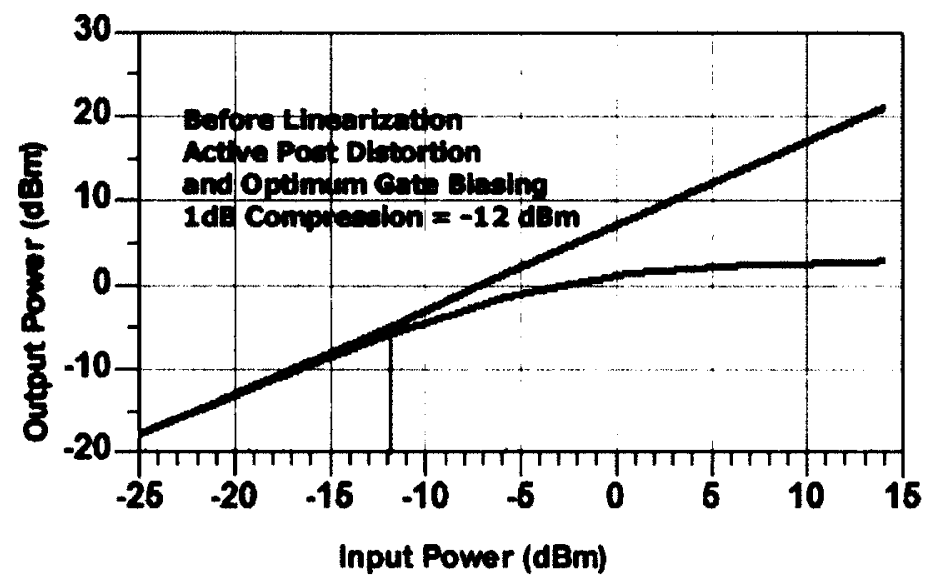

Figure 4.16: Simulated 1-dB gain compression point before linearization for the proposed linearized CMOS distributed $2 \times 3$ matrix amplifier.

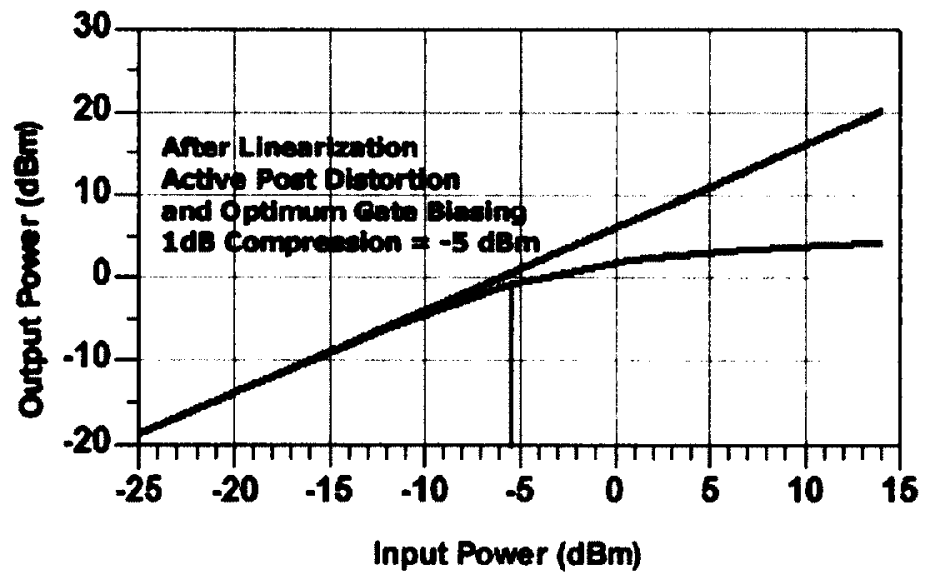

Figure 4.17: Simulated 1-dB gain compression point after linearization for the proposed linearized CMOS interleaved distributed 2 × 3 matrix amplifier. 


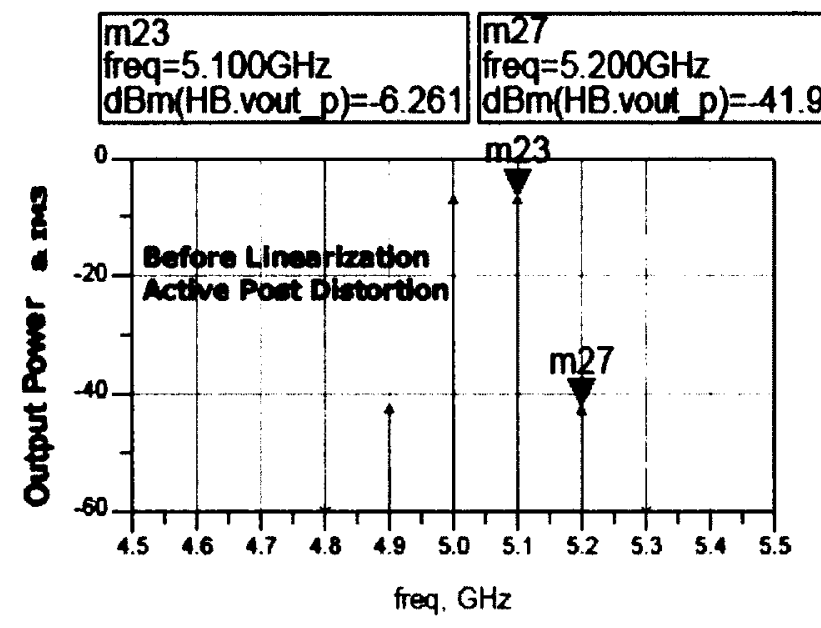

Figure 4.18: Simulated Two-Tone before active post distortion linearization only at $5 \mathrm{GHz}$ for the proposed linearized CMOS interleaved distributed $2 \times 3$ matrix amplifier.

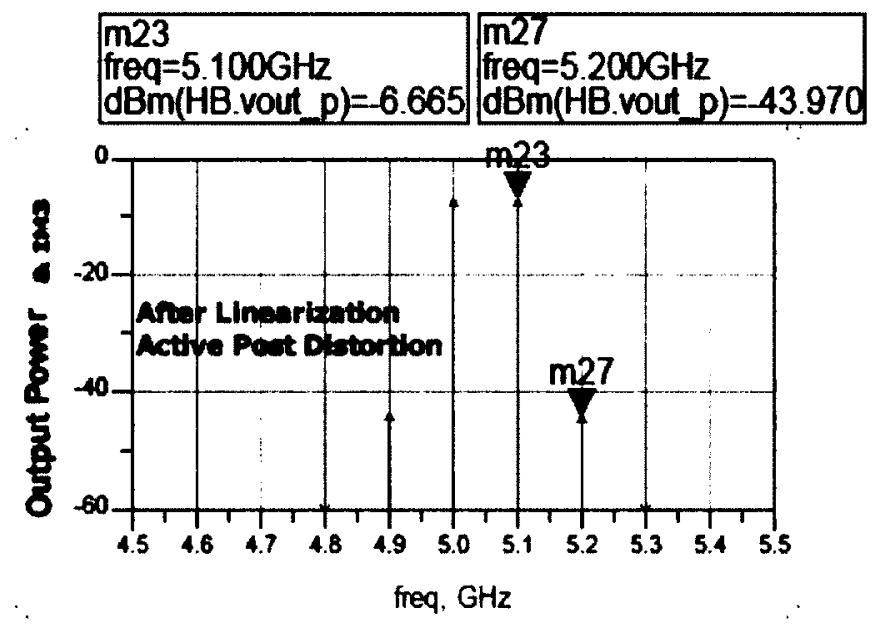

Figure 4.19: Simulated Two-Tone after active post distortion linearization only at $5 \mathrm{GHz}$ for the proposed linearized CMOS interleaved distributed $2 \times 3$ matrix amplifier. 
transconductance $g_{m}$ peak of one transistor can be canceled by the positive peak of an auxiliary MOSFET with an optimum gate voltage offset bias so that the magnitude of the its positive third-order transconductance $g_{m}$ peak is equal to the negative peak of the master transistor [25]. The combined transistors yield a third-order transconductance nonlinearity near-zero and in turn improves its linearity. It can be concluded from simulations that tuning the linearized CMOS distributed $2 \times 3$ matrix amplifier using active post distortion and optimum gate bias improves its broadband linearity and in turn improves its dynamic range as well. As can be seen in Figure 4.16 and Figure 4.17 by shifting the bias point and superposing the device characteristics the third-order nonlinearity can be canceled and 1-dB gain compression point is improved as well.

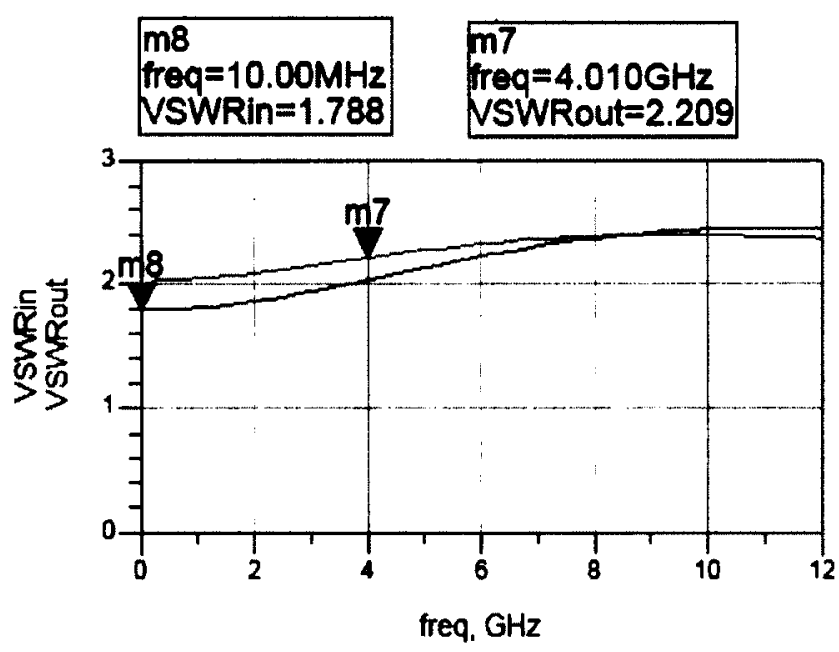

Figure 4.20: The VSWR for the proposed linearized CMOS interleaved distributed $2 \times 3$ matrix amplifier.

The varactor-based active post distortion enables one to tune the IM3 suppression as can be seen in Figure 4.18 and Figure 4.19, it provides repeatable electronically tunable distortion cancellation at various combination of varactor voltages. The VSWR voltage standing wave ratio for the proposed Linearized CMOS Interleaved Distributed $2 \times 3$ Matrix Amplifier is shown in Figure 4.20. 


\subsection{Linearized CMOS Distributed Paraphase Ampli- fier}

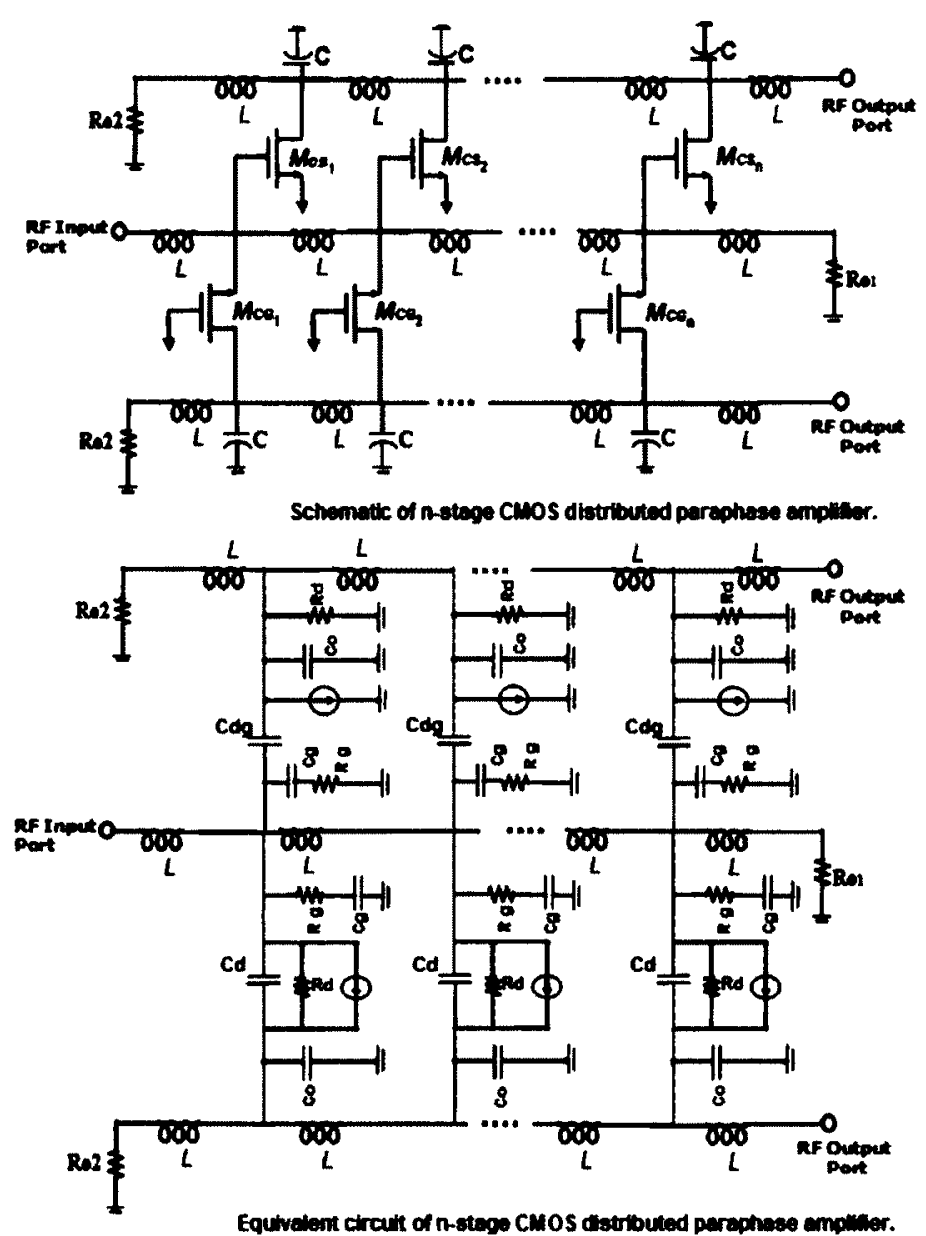

Figure 4.21: Block diagram illustration of CMOS distributed paraphase amplifier.

Broadband paraphase amplifiers find many applications in microwave applications and wireless communications. Circuits which are suitable for fully monolithic integration in cost effective CMOS technology are of particular interest [96]. Sokolov and Williams [97] have reported on a narrowband MMIC GaAs paraphase amplifier at $8.5 \mathrm{GHz}$ and LeventVillegas [98] has reported on another narrowband GaAs paraphase amplifier over the range 
of $9-11 \mathrm{GHz}$.

A fully-integrated CMOS linearized distributed paraphase amplifier (unbalanced input, balanced output) is presented in this section. The CMOS linearized distributed paraphase amplifier or linearized active balun will provide two outputs, 180 degree out of phase over a wide microwave band with minimal phase and amplitude imbalance. Compared to passive balun circuits, the linearized CMOS distributed paraphase amplifier's advantage is that it provides gain and allows for broadband distortion cancellation. The proposed CMOS linearized distributed paraphase amplifier makes up for the inherent $3 \mathrm{~dB}$ loss of balun and be used to drive balanced antennas and wideband phase shifters.

The wideband distributed paraphase amplifier is based on the concept of the distributed amplifier ( [27], [85]). Figure 4.21 shows a schematic of an n-stage distributed paraphase amplifier and its equivalent circuit. It consists of $n$-stage distributed FET amplifiers. One is a distributed common-source amplifier, the other is a distributed common-gate amplifier. These two amplifiers share a common input transmission line. A simple circuit model for the distributed paraphase amplifier is shown Figure 4.21.

The power gain of the common-gate section of the distributed paraphase amplifier shown in Figure 4.21 is given by [31]

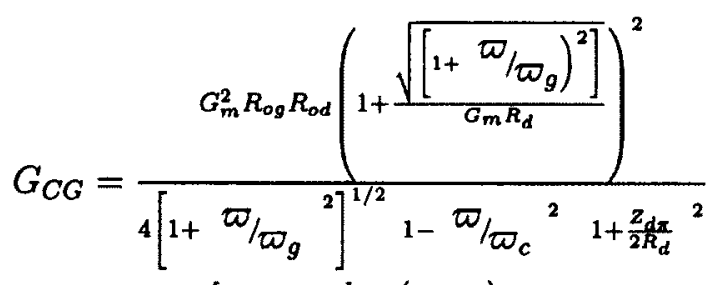

$$
\begin{aligned}
& \times \frac{\sinh ^{2}\left[\frac{n}{2}\left(\alpha_{d}-\alpha_{g}\right)\right] e^{-n\left(\alpha_{d}+\alpha_{g}\right)}}{\sinh ^{2}\left[\frac{1}{2}\left(\alpha_{d}-\alpha_{g}\right)\right]}
\end{aligned}
$$

where $G_{m}$ is the transconductance and $\alpha_{d}$ and $\alpha_{g}$ are the attenuation per section and $R_{o d}$ and $R_{o g}$ are the characteristic resistance and $\varpi_{d}$ and $\varpi_{g}$ are of the drain and gate line respectively.

The power gain of the distributed common-source amplifier in the distributed paraphase amplifier shown in Figure 4.21 is given by ( [31], [29]) 


$$
\begin{gathered}
G_{C S}=\frac{G_{m}^{2} R_{o g} R_{o d}}{4\left[1+\varpi_{\varpi_{g}}\right]^{2} 1-\varpi_{/ \varpi_{c}}{ }^{1 / 2} 1-} \\
\quad \times \frac{\sinh ^{2}\left[\frac{n}{2}\left(\alpha_{d}-\alpha_{g}\right)\right] e^{-n\left(\alpha_{d}+\alpha_{g}\right)}}{\sinh ^{2}\left[\frac{1}{2}\left(\alpha_{d}-\alpha_{g}\right)\right]}
\end{gathered}
$$

where $R_{o d}$ and $R_{o g}$ are the characteristic resistance and $G_{m}$ is the transconductance.

The amplitude imbalance of the distributed paraphase amplifier can be expressed as [31]

$$
\begin{gathered}
\Delta A(d B)=20 \log \frac{1+\frac{1}{G_{m} R_{d}}}{1+\frac{Z_{d x}}{2 R_{d}}}+20 \log \frac{\cosh \left(\frac{n}{2} \frac{\alpha_{d}}{\alpha_{o}}\right)}{\cosh \left(\frac{1}{2} \frac{\alpha_{d}}{\alpha_{o}}\right)} \\
-10 \log \left[\exp \left(n \frac{\alpha_{d}}{\alpha_{o}}\right)\right]
\end{gathered}
$$

where $G_{m}$ is the transconductance. The first term results from the gain difference between the two stages while the latter two terms stem from the difference in attenuation on the two output lines.

The phase imbalance of more practical concern is given by [31]

$$
\delta \phi=\arctan \frac{\varpi C_{d}}{\frac{1}{R_{d}}+G_{m}}-\arctan \frac{\varpi C_{d}}{\frac{1}{R_{d}}+\frac{2}{Z_{d \pi}}}
$$

where the $G_{m}$ is the device transconductance. The design parameters such as the output line impedance is dictated by circuit constraints the device can be chosen to minimize the phase imbalance.

\subsubsection{Amplitude and Phase Imbalance of Linearized CMOS Dis- tributed Paraphase Amplifier}

The inherent broadband characteristics of the distributed amplifier is applied to a linearized CMOS distributed paraphase design. In the amplifier a distributed common-gate amplifier and a distributed common-source amplifier are fed by a single input transmission line. The characteristic low pass response of the distributed amplifier then yields two RF outputs from one RF input signal, the amplitudes of both RF output signals are equal and their phase difference is 180 degree over a wide band ( [79], [80], [99]). Figure 4.25 shows the schematic of the proposed linearized CMOS distributed paraphase amplifier. A distributed pre-amplifier stage is added which not only supplies gain but serves as an active impedance transformer. 


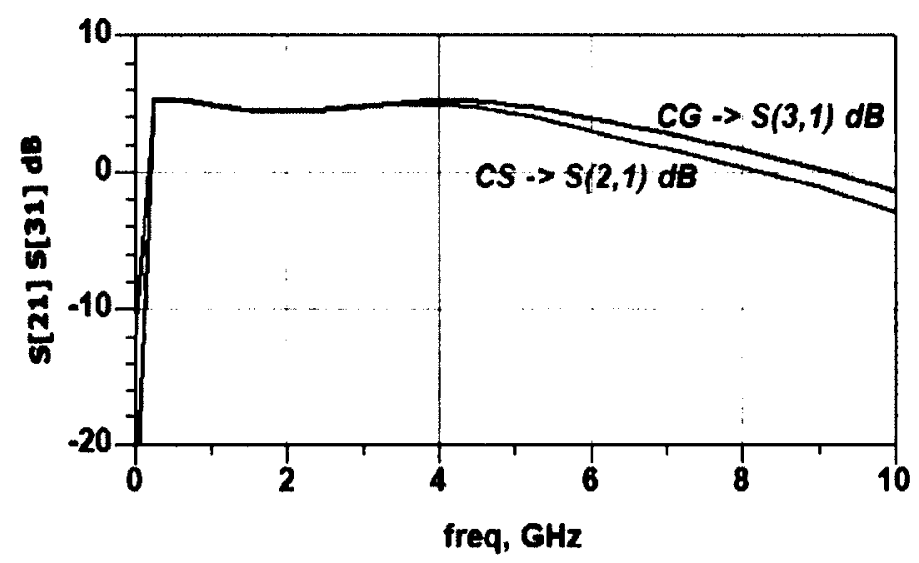

Figure 4.22: Simulated Output Power S21 common-source stage port and S31 commongate stage port peaks at 5.3 $\mathrm{dB}$ for the proposed Linearized CMOS Distributed Paraphase Amplifier.

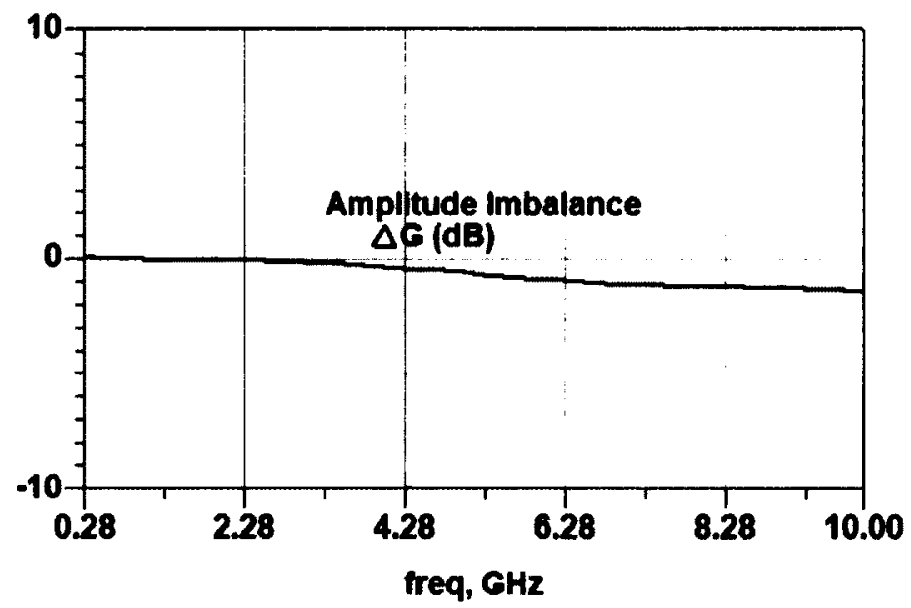

Figure 4.23: Simulated amplitude imbalance for the proposed linearized CMOS distributed paraphase amplifier amplitude imbalance of less than $1.26 \mathrm{~dB}$. 


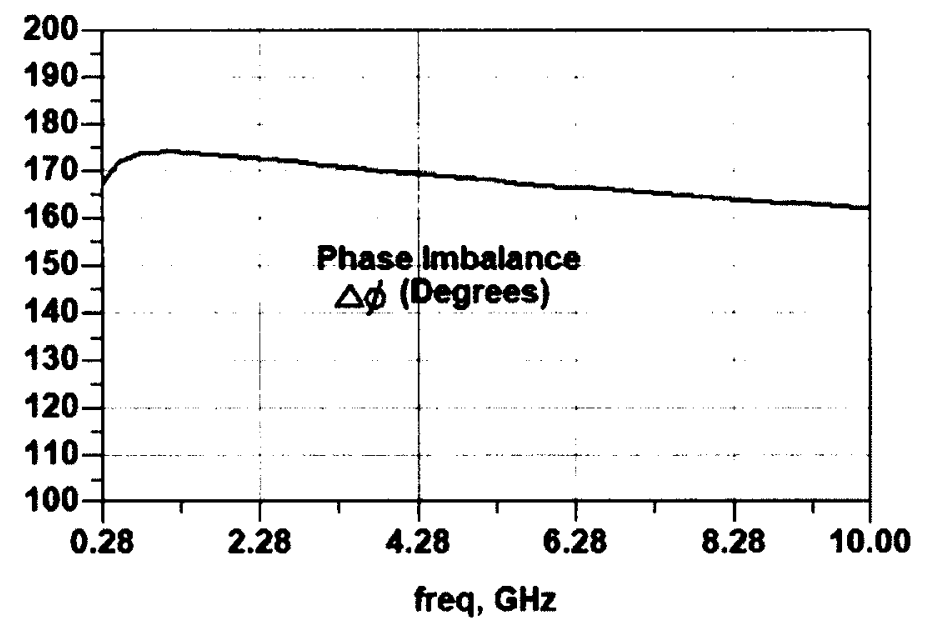

Figure 4.24: Simulated phase imbalance for the proposed linearized CMOS distributed paraphase amplifier with less than 10 degrees phase imbalance.

The lumped transmission lines tend to provide a constant input and output resistance over a wide passband. As a result, the phase difference between the output of the commongate amplifier and the output of the common-source amplifier is theoretically 180 degree, independent of the frequency, if the phase velocities of the signals on the three transmission lines are identical. The voltage gains of the common-source amplifier and the common-gate amplifier are almost the same with nearly equal output power as can be seen in Figure 4.22 and as a result, a wideband CMOS distributed paraphase amplification is obtained.

The linearized CMOS distributed paraphase amplifier exhibiting an $5.3 \mathrm{~dB}$ small signal $S_{21}$ power gain peak as shown in Figure 4.22 and rolls off to a unity gain bandwidth of $8.2 \mathrm{GHz}$ with less than 10 degrees phase imbalance as shown in Figure 4.24 and amplitude imbalance of less than $1.26 \mathrm{~dB}$ over the band as shown in Figure 4.23.

\subsubsection{CMOS Distributed Paraphase Amplifier Employing Deriv- ative Superposition Linearization}

The principle of derivative superposition is to eliminate the considerable contribution to the overall amplifier distortion originating from the transconductance $G_{m}$ nonlinearity ( $[100]$, 


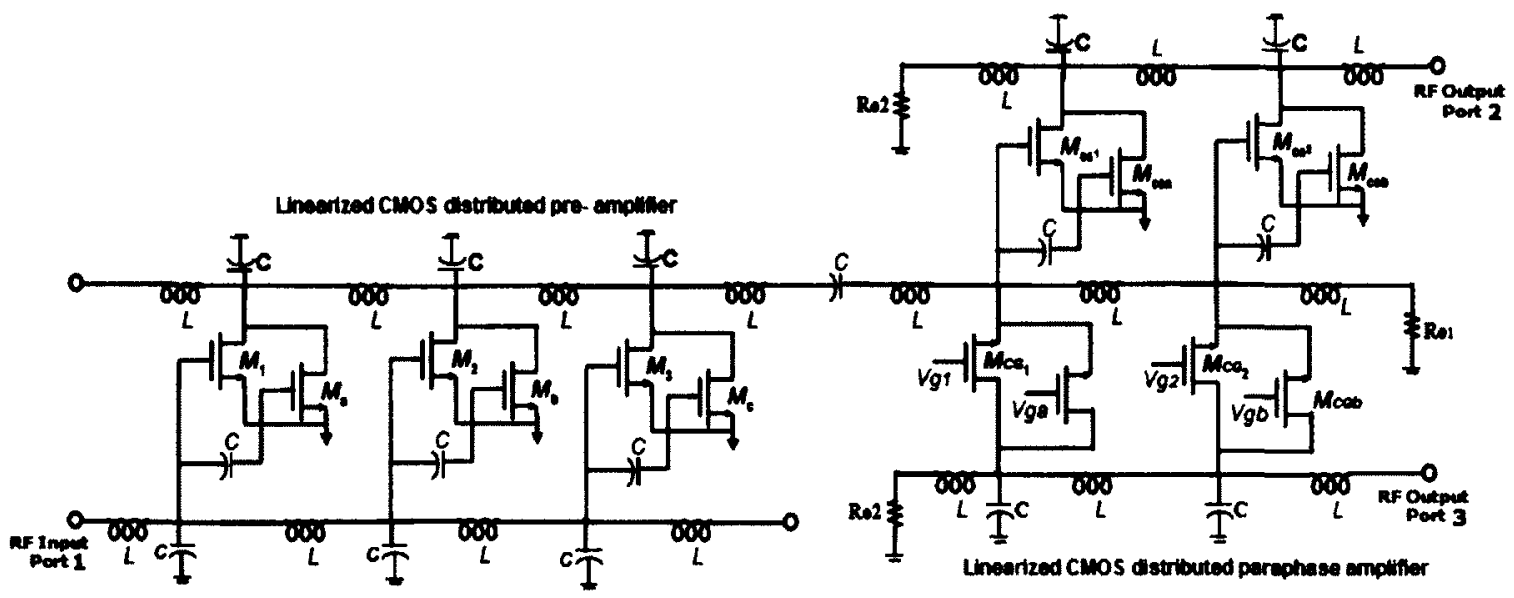

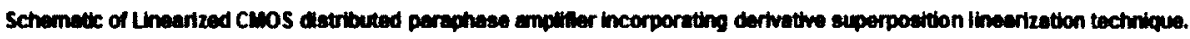

Figure 4.25: Schematic Topology of the Proposed Fully-Integrated Linearized CMOS Distributed Paraphase Amplifier.

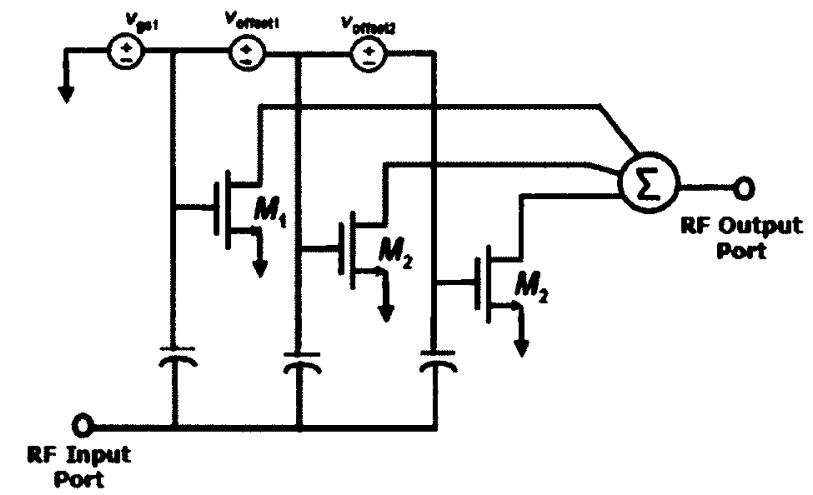

Schematic of Lineartzed CMOS common source ampllier incorporating derivative superposition linearization technique.

Figure 4.26: The derivative superposition linearization approach. The overall linearized transconductance is composed out of several gate bias shifted common source FET devices, which are current summed at their outputs. 
[101]). By running different sized transistors in parallel the direct current operating point of each single device is adjusted to synthesize a transfer function which does not generate any nonlinearities. The drain and gate transmission-line inductors have an inductance value of $1.2 \mathrm{nH}$ and the drain transmission-line $\mathrm{m}$-derived inductance is $110 \mathrm{pH}$ with 50 Ohms terminations. The device dimensions $\left[M_{1}, M_{3}\right](\mathrm{W} / \mathrm{L})$ and $\left[M_{c s 1}, M_{c s 2}\right](\mathrm{W} / \mathrm{L})$ have a width equal to $48 \mu \mathrm{m}$ with all devices having $\mathrm{L}$ minimum channel length of $120 \mathrm{~nm}$. The device dimensions $\left[M_{a}, M_{c}\right](\mathrm{W} / \mathrm{L})$ and $\left[M_{c s a}, M_{c s b}\right](\mathrm{W} / \mathrm{L})$ have a width equal to $24 \mu \mathrm{m}$ with all devices having $L$ minimum channel length of $120 \mathrm{~nm}$.

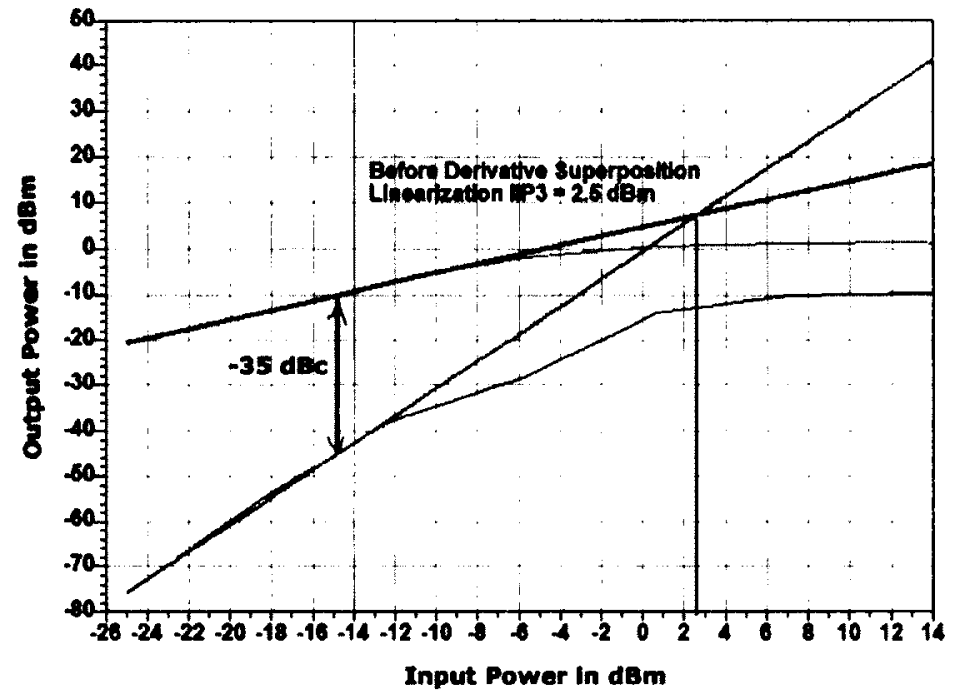

Figure 4.27: ADS Simulation of IIP3 before CMOS distributed paraphase amplifier derivative superposition linearization.

The derivative superposition technique [66] is based on the drain current dependence versus $V_{g s}$. When considering the related 3rd order Taylor coefficient of this current gm3, which represents the third order nonlinearity, this bias dependence results in magnitude and phase reversals for gm3 in the threshold region. The generalized circuit topology to be used with derivative superposition is shown in Figure 4.26.

In this section the derivative superposition linearization technique is applied to the linearized CMOS distributed paraphase design. The tuning of the power series nonlinear 


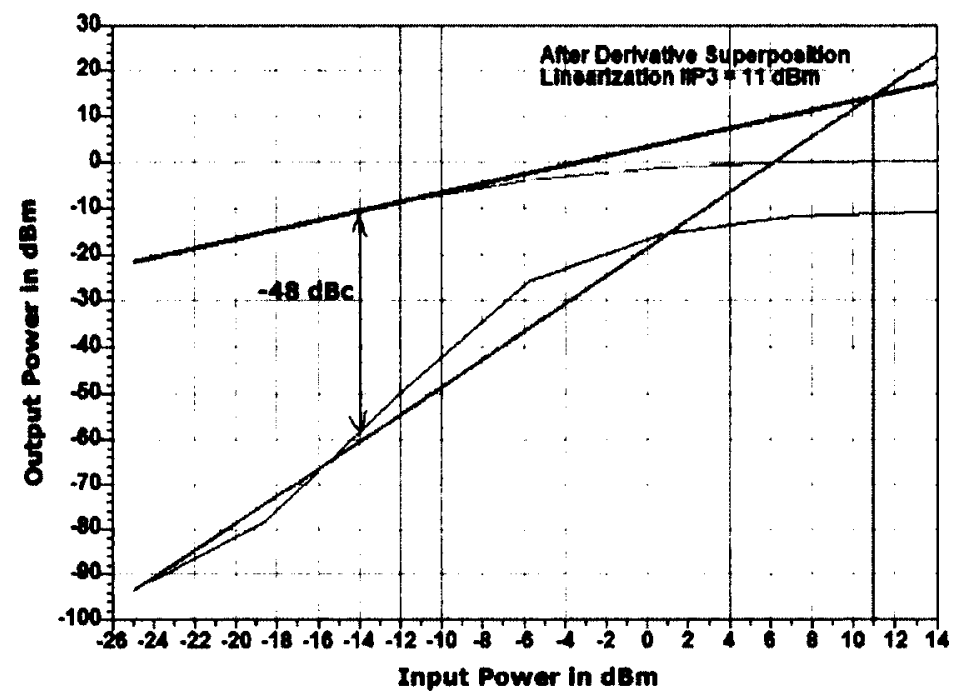

Figure 4.28: ADS Simulation of IIP3 after CMOS distributed paraphase amplifier derivative superposition linearization with a $8.5 \mathrm{~dB}$ and a $13 \mathrm{dBc}$ improvement at output power of $-10 \mathrm{dBm}$.

coefficient, transconductance gm, and its effect on IIP3 is shown in Figure 4.27 and Figure 4.28. It can be concluded from simulations that tuning the linearized CMOS distributed paraphase amplifier using derivative superposition improves its broadband linearity and in turn improves its dynamic range as well. As can be seen in Figure 4.27 and Figure 4.28 by shifting the bias point and superposing the device characteristics the third-order nonlinearity can be canceled. Both $8.5 \mathrm{~dB}$ IIP3 improvement and $13 \mathrm{dBc}$ improvement, at output power of $-10 \mathrm{dBm}$, can be seen in Figure 4.28 .

\subsection{Chapter Summary}

The interest in highly-linear multi-functional broadband subsystems motivated the development of linearized distributed circuit applications. In this chapter, various applications of linearized distributed circuit functions such as fully-integrated CMOS linearized distributed active power splitter (unbalanced input, balanced output) and a linearized CMOS 
distributed matrix amplifier were presented. Also the linearized CMOS distributed paraphase amplifier employing derivative superposition linearization was presented as well. The proposed fully-integrated linearized distributed circuit designs are suitable for highly-linear broadband wireless transceiver applications. 


\section{Chapter 5}

\section{Linearized CMOS Distributed Bidirectional Amplifier with Cross-Coupled Compensator}

\subsection{Introduction}

In this chapter, we demonstrate a fully-integrated fully-differential linearized CMOS distributed bidirectional amplifier that achieves large IMD3 distortion reduction over broadband frequency range for both RF paths. The drain and gate transmission-lines were stagger-compensated. Reducing the DA IM3 distortion by mismatching the gate and drain LC delay-line ladders. A CMOS cross-coupled compensator transconductor is proposed to enhance the linearity of the DA gain cell with a varactor-based active post nonlinear drain capacitance compensator for wider linearization bandwidth.

\subsection{Linearized CMOS Distributed Bidirectional Am- plifier Circuit Design Analysis}

For wireless base station transmitters in wireless communications, high linearity and broadband bandwidth are some of the primary system requirements. Linear broadband amplification is required in order to reduce signal spectral regrowth. The wide bandwidth of the DA structure makes it an attractive component for use in systems requiring broadband operation. A detailed paper by Ginzton et al. [56] implemented Percival's patented concept 
of distributed amplification [75]. Conventional DAs use low-pass $\pi$-sections to form an artificial transmission-line topology. Amplification gain stages are connected so that output currents are combined in an additive manner at the output terminal. The advantages of a DA topology are its wide bandwidth, flat gain and compact size circuit size. A three-stage CMOS DA with MOSFET devices is shown in Figure 5.1. The major three nonlinear elements of the MOSFET devices are $g_{m}, C_{d}$ and $C_{g s}$. An ideal broadband amplifier should be a totally linear device. But in real world, amplifiers are only linear within certain practical limits. When the input signal driven into the amplifier is increased, the output is also increased until a point where distortion products can no longer be ignored. The harmonics of the output signal are generated by nonlinearities of the MOSFET devices. The nonlinear element transconductance $g_{m}$ is a function of $V_{g}$ and can be expressed by power series with coefficients $g_{m 1}, g_{m 2}$ and $g_{m 3}$ as in equation 5.2.1.

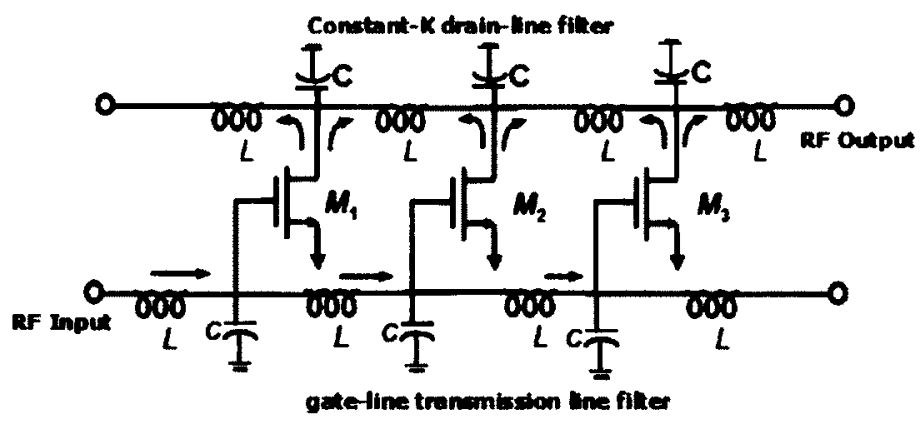

Figure 5.1: A three-stage CMOS DA with MOSFET devices.

The characteristic of the nonlinear transconductance can be approximated by a threeterm power series expansion for each MOSFET device in Figure 5.1 ( [13], [16], [38], [84])

$$
i_{d}=g_{m 1} v_{g s}+g_{m 2} v_{g s}^{2}+g_{m 3} v_{g s}^{3}
$$

As the input signal increases the instantaneous value of $g_{m}$ changes with the level of the input signal and the amplifier operating into its nonlinear region. We can write $v_{g s}$ as $([85],[102])$ 


$$
v_{g s}=V \cos (\omega t)
$$

where $\mathrm{V}$ is the amplitude signal and substituting equation 5.2 .2 into 5.2.1, the expression for the current at frequency $\omega$ is given by ( [86], [15])

$$
i=g_{m 1} V \cos (\omega t)+\frac{3}{4} g_{m 3} V^{3} \cos (\omega t)
$$

In the three-stage CMOS DA shown in Figure 5.1, the fundamental component of the output current from each stage can be defined as ( [15], [103], [104])

$$
\begin{aligned}
& i_{1}=g_{m 1} V \cos [\omega t] e^{-j 1 \beta_{g}} \\
& i_{2}=g_{m 1} V \cos [\omega t] e^{-j 2 \beta_{g}} \\
& i_{3}=g_{m 1} V \cos [\omega t] e^{-j 3 \beta_{g}} \\
& i_{k}=g_{m 1} V \cos [\omega t] e^{-j k \beta_{g}}
\end{aligned}
$$

where $\beta_{g}$ is the phase constant of the gate line.

The total fundamental output current delivered to the load for all three-stages can be found as ([15], [105])

$$
I_{\text {linear }}=\frac{1}{2}\left(i_{1} e^{-j n \beta_{d}}+i_{2} e^{-j(n-1) \beta_{d}}+i_{3} e^{-j(n-2) \beta_{d}}\right)
$$

where $\beta_{d}$ is the phase constant of the drain line and $\mathrm{n}$ is a constant representing the number of stages.

In the three-stage CMOS DA as shown in Figure 5.1, the third-order intermodulation IM3 component of the output current from each stage can be defined as

$$
i_{m 1}=\frac{3}{4} g_{m 3} V^{3} \cos [\omega t] e^{-j 3 \beta_{g}}
$$




$$
\begin{aligned}
& i_{m 2}=\frac{3}{4} g_{m 3} V^{3} \cos [\omega t] e^{-j 6 \beta_{g}} \\
& i_{m 3}=\frac{3}{4} g_{m 3} V^{3} \cos [\omega t] e^{-j 9 \beta_{g}} \\
& i_{m k}=\frac{3}{4} g_{m 3} V^{3} \cos (\omega t) e^{-j 3 k \beta_{g}}
\end{aligned}
$$

The corresponding total $I M_{3}$ output current delivered to the load for all three-stages can be found as

$$
I_{I M 3}=\frac{1}{2}\left(i_{m 1} e^{-j n \beta_{d}}+i_{m 2} e^{-j(n-1) \beta_{d}}+i_{m 3} e^{-j(n-2) \beta_{d}}\right)
$$

Dividing equation 5.2 .8 by 5.2 .13 yields

$$
I_{\text {linear }} / I_{I M 3}=\frac{4}{3} e^{6 R e\left[j \beta_{g}\right]}\left|\frac{\left(e^{2 j \beta_{d}}+e^{2 j \beta_{g}}+e^{j\left(\beta_{d}+\beta_{g}\right)}\right) g_{m 1}}{\left(e^{2 j \beta_{d}}+e^{6 j \beta_{g}}+e^{j\left(\beta_{d}+3 \beta_{g}\right)}\right) g_{m 3} V^{2}}\right|
$$

It can be seen from Equation 5.2.14 as well that the carrier power to third-order intermodulation $C / I M_{3}$ is dependent on the power series nonlinear coefficients $g_{m 1}$ and $g_{m 3}$ and that $C / I M_{3}$ can be improved by reducing the $g_{m 3}$ nonlinearity.

Equation 5.2.14 can be extended to an n-stage CMOS DA. The total fundamental output current delivered to the load for $n$-stages can be found as

$$
I_{\text {linear }}=\frac{1}{2} \sum_{r=1}^{n} g_{m 1} V \cos [\omega t] e^{-j r \beta_{g}} e^{-j(n-r+1) \beta_{d}}
$$

The total fundamental output current delivered to the load for n-stages can be simplified as

$$
I n_{\text {linear }}=\frac{e^{-j n \beta_{d}}\left(-1+\left(e^{j\left(\beta_{d}-\beta_{g}\right)}\right)^{n}\right) g_{m 1} V \operatorname{Cos}[t \omega]}{2\left(e^{j \beta_{d}}-e^{j \beta_{s}}\right)}
$$

The corresponding total $I M_{3}$ output current for all n-stages flowing through the drain load can be defined as

$$
I n_{I M 3}=\frac{1}{2} \sum_{r=1}^{n} \frac{3}{4} g_{m 3} V^{3} \operatorname{Cos}[\omega t] e^{-j 3 r \beta_{g}} e^{-j(n-r+1) \beta_{d}}
$$


The total $\mathrm{IM}_{3}$ output current for n-stages flowing through the drain load can be simplified as

$$
I n_{I M 3}=\frac{3 e^{-j n \beta_{d}}\left(-1+\left(e^{j\left(\beta_{d}-3 \beta_{g}\right)}\right)^{n}\right) g_{m 3} V^{3} \operatorname{Cos}[t \omega]}{8\left(e^{j \beta_{d}}-e^{3 j \beta_{g}}\right)}
$$

Dividing equation 5.2 .16 by 5.2 .18 yields

$$
I n_{\text {linear }} / I n_{I M 3}=\frac{4}{3} \frac{\left(e^{j \beta_{d}}-e^{3 j \beta_{g}}\right)\left(-1+\left(e^{j\left(\beta_{d}-\beta_{g}\right)}\right)^{n}\right) g_{m 1}}{\left(e^{j \beta_{d}}-e^{j \beta_{g}}\right)\left(-1+\left(e^{j\left(\beta_{d}-3 \beta_{g}\right)}\right)^{n}\right) g_{m 3} V^{2}}
$$

From Equation 5.2.16 the overall fundamental filter frequency response is expressed as

$$
F(s)_{f u n d}=\frac{e^{-j n \beta_{d}}\left(-1+\left(e^{j\left(\beta_{d}-\beta_{g}\right)}\right)^{n}\right)}{2\left(e^{j \beta_{d}}-e^{j \beta_{g}}\right)}
$$

From equation 5.2.18 overall IM3 filter frequency response is expressed as

$$
F(s)_{i m 3}=\frac{3 e^{-j n \beta_{d}}\left(-1+\left(e^{j\left(\beta_{d}-3 \beta_{g}\right)}\right)^{n}\right)}{8\left(e^{j \beta_{d}}-e^{3 j \beta_{g}}\right)}
$$

where the phase constant of the gate line is given by $\beta_{g}=\omega \sqrt{L_{g} \cdot C_{g}}$ and $\beta_{d}=\omega \sqrt{L_{d} \cdot\left(C_{d}+C_{v a r}\right)}$ is the phase constant of the drain line.

The characteristic impedance of the drain line is given by $Z_{o-d}=\sqrt{\frac{C_{d}}{L_{d}}}$ and the drain cut-off frequency is given by $\omega_{c}=\frac{1}{\sqrt{L_{d} C_{d}}}$

Designing a staggered bidirectional DA with mismatched time-delay for the drain and gate line can be achieved by mismatching the drain and gate line capacitance. A simulation of a staggered drain and gate transmission-lines with mismatched time-delay to filter out the IM3 distortion improving the $C / I M_{3}$ in broadband CMOS bidirectional DAs is shown in Figure $5.2(\mathrm{a})$. Further increase in the time-delay mismatch will shift the $I M_{3}$ attenuation frequency response inside the in-band of the DA cutoff frequency as can be seen in Figure 5.2 (b) and Figure 5.3. Thus, having a staggered distributed structure helps in improving the carrier power to third-order intermodulation distortion power ratio $C / I M_{3}$ in broadband CMOS amplifiers [30]. 

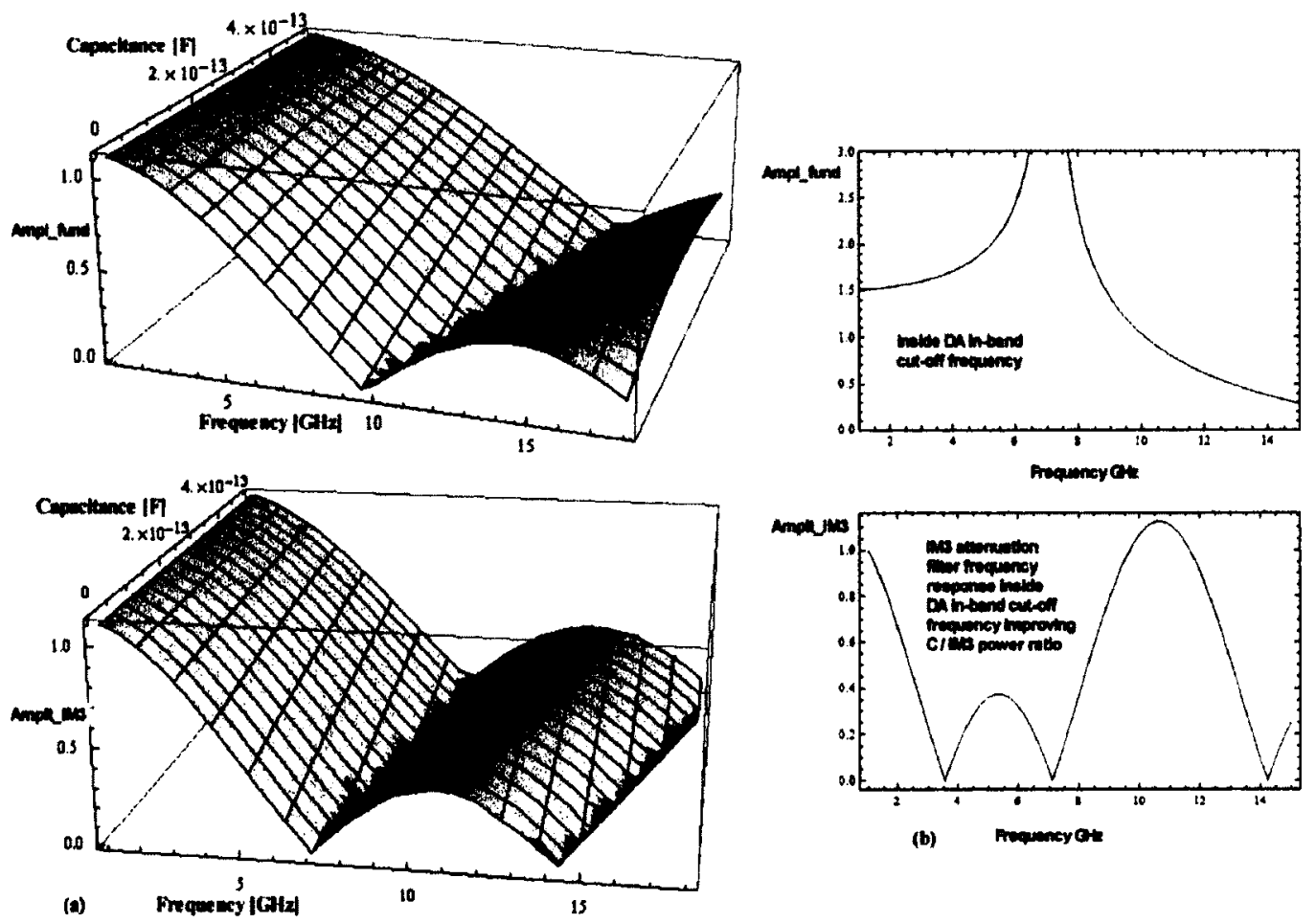

Figure 5.2: (a) Designing a staggered bidirectional DA structure with mismatched timedelay for the drain and gate line will shift the IM3 attenuation frequency response inside the in-band of the DA cut-off frequency and in turn out the IM3 distortion (b) Further increase in the time-delay mismatch will shift the $I M_{3}$ attenuation frequency response inside the in-band of the DA cutoff frequency.

\subsection{CMOS Cross-Coupled Compensator Transconduc- tor as DA Gain Cell for Linearity Improvement and Enhanced Tunability}

In radio transceivers, a differential pair transconductor is often used to convert the RF input voltage signal to a current that is either amplified or frequency translated ( [33], [36]). External linearization circuitry can be added to the differential pair amplifier in order to compensate for the transconductance nonlinearity [60]. This allows the nonlinear amplifier 

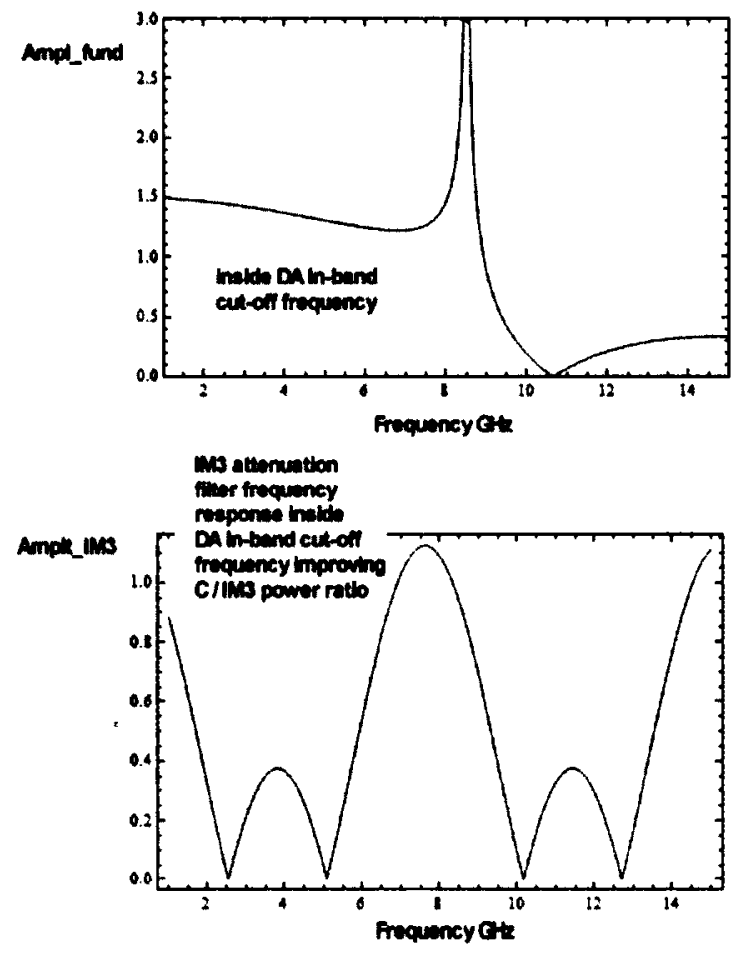

Figure 5.3: More increase in time delay mismatch by increasing the drain and gate line capacitance mismatching will shift the IM3 attenuation frequency response even more inside the in-band of the distributed amplifier cut-off frequency further filtering out IM3 distortion.

to be used to amplify signals utilizing spectrally efficient linear modulation techniques without causing interference ( [34], [36]). Many techniques have been developed to improve the linearity of the basic differential pair transconductor such as source degeneration, Caprio's cross-quad [106] and Quinn's cascomp transconductor [107]. However, their second derivative device transconductor transistor parameter gm" nulling is not sufficiently wide and their device transconductor parameter gm flatness is limited. Other published linearized BJT V-I converters such as proposed in [81] does not linearize the total transconductor drain current instead only linearizing the inner translinear loop current with no on-chip tuning. The proposed linearized cross-coupled compensator transconductor achieves a wide tunable gm" nulling and increase in device transconductor parameter gm flatness. 
In this chapter, we propose a $0.13 \mathrm{um}$ RF CMOS highly-linear differential cross-coupled compensator transconductor that combines both cross-quad and cascomp linearization techniques with enhanced tunability [81]. The proposed cross-coupled compensator linearize the total drain current while maintaining a high input voltage swing range. It achieves a wide tunable second derivative device transconductor parameter gm" nulling over $500 \mathrm{mV}$ differential input signal and a tunable 50 percent increase in the device transconductor parameter gm flatness. The proposed linearized transconductor offers significant improvement in linearity for the use in highly-linear broadband high-frequency amplifiers [30]. The proposed cross-coupled compensator transconductor [100] maintains a high input voltage swing range due to the aid of the second translinear loop that is not implemented in the other published BJT V-I converter in [81].

The proposed differential CMOS cross-coupled compensator transconductance is implemented as shown in Figure 5.4 (a). A source degeneration resistor $R_{b}$ is placed across the outer cascode differential pair in between the identical current sources Ibias $s_{b}$. Similarly a source degeneration resistor $R_{t}$ is placed in between current source Ibias $_{t}$ inner cascode differential pair. To achieve a distortion-less V-I linear conversion of the transconductor total current $\Delta I_{\text {out }}$, both bias current sources $I_{\text {bias }}$ and $I_{b i a s_{t}}$ are tuned to ensure both currents $\Delta I_{t}$ and $\Delta I_{b}$ become linearly proportional to the input voltage $\Delta V_{\text {in }}$. Hence the proposed CMOS cross-coupled compensator total current $\Delta I_{\text {out }}=\Delta I_{t}+\Delta I_{b}$ is linearly proportional to the input voltage $\Delta V_{\text {in }}$ contrary to the linearized BJT transconductor in [108]. In the linearized BJT transconductor the inner translinear loop only considers the inner loop current $\Delta I_{\text {inner }}$ which becomes linearly proportional to the input voltage $\Delta V_{\text {in }}=(2 R e e) . \Delta I_{\text {inner }}$ and does not consider the total current $\Delta I_{\text {out }}$ of the transconductor.

Lets consider the cross-coupled compensator sum of voltages around the first translinear loop comprising the signal generator and gate-source voltages of $\left(M_{1}, M_{9}, M_{10}, M_{2}\right)$ and the degeneration resistor $R_{t}$ as shown in Figure 5.4 (a). Ignoring body effects yields the following expression:

$$
\Delta I_{t}=\frac{\Delta V_{i n}-\left(V_{G S 1}+V_{G S 9}-V_{G S 10}-V_{G S 2}\right)}{R_{t}}
$$

Next, consider the sum of the voltages around the second translinear loop comprising the gate-source voltages of $\left(M_{3}, M_{7}, M_{8}, M_{4}\right)$ and the degeneration resistor $R_{b}$ as shown 


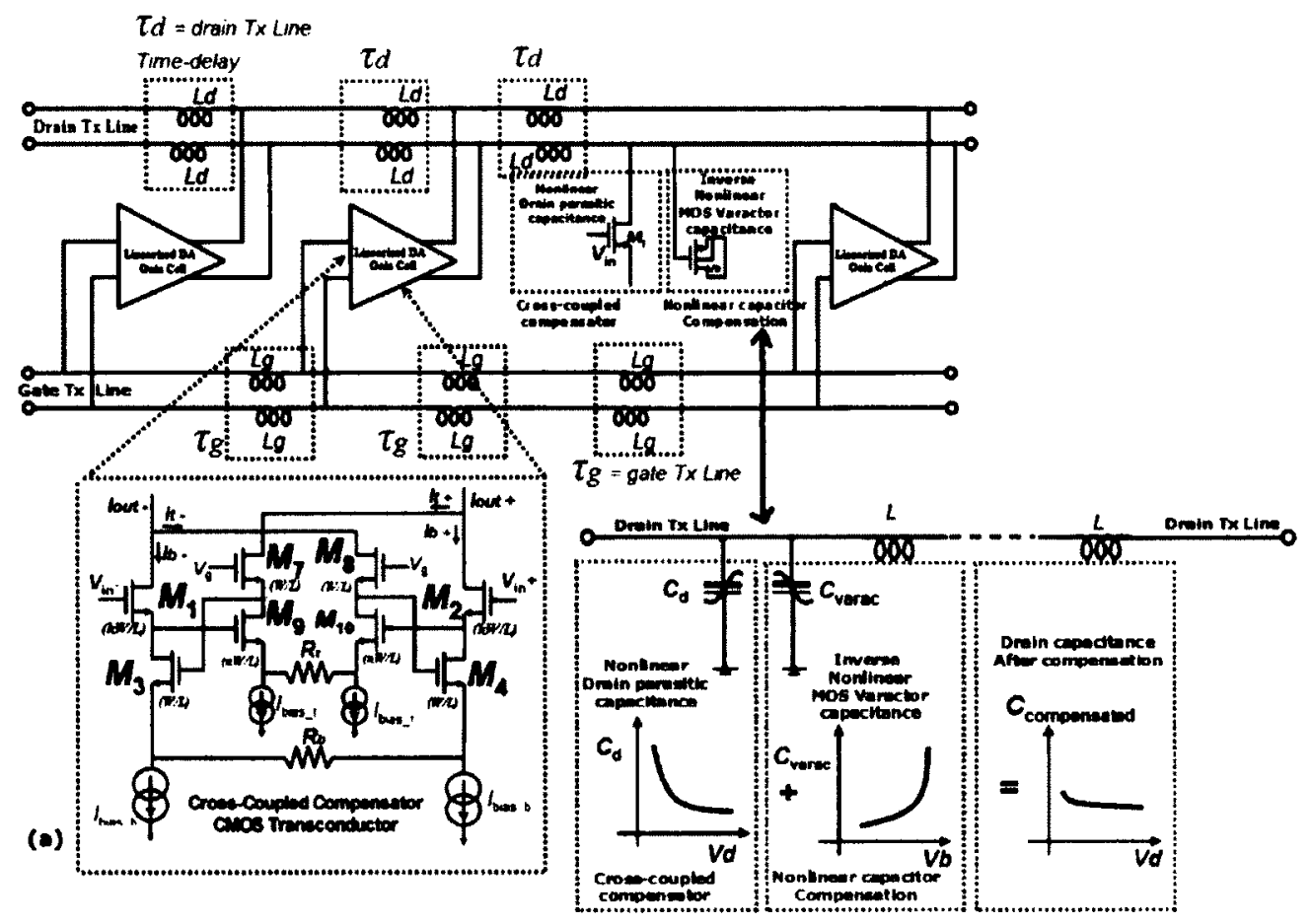

(b) Drain $\mathrm{T} x$ line nonlinear perasitic capachance componeadon

Figure 5.4: (a) Proposed topology of the three-stage bidirectional distributed amplifier with CMOS cross-coupled compensator transconductor gain cells coupling the staggered drain and gate transmission-lines with nonlinear drain capacitance compensator (b) A varactor-based active post nonlinear drain capacitance compensator in the CMOS distributed structure for wider linearization bandwidth.

in Figure 5.4 (a). Ignoring body effects yields the following expression:

$$
\Delta I_{b}=\frac{\left(V_{G S 3}+V_{G S 7}-V_{G S 8}-V_{G S 4}\right)}{R_{b}}
$$

Adding both loops together yields the total current $\Delta I_{\text {out_total }}$ :

$$
\begin{gathered}
\Delta I_{\text {out_total }}=\Delta I_{t}+\Delta I_{b} \\
\Delta I_{\text {out_total }}=\frac{\Delta V_{\text {in }}-\left(V_{G S 1}+V_{G S 9}-V_{G S 10}-V_{G S 2}\right)}{R_{t}}+\frac{\left(V_{G S 3}+V_{G S 7}-V_{G S 8}-V_{G S 4}\right)}{R_{b}}
\end{gathered}
$$




$$
\Delta I_{\text {out_total }}=\frac{\Delta V_{\text {in }}}{R_{t}}-\frac{\left(V_{G S 1}+V_{G S 9}-V_{G S 10}-V_{G S 2}\right)}{R_{t}}+\frac{\left(V_{G S 3}+V_{G S 7}-V_{G S 8}-V_{G S 4}\right)}{R_{b}}
$$

For the total differential output current $\Delta I_{\text {out }}$ to achieve a linear relationship with the input differential voltage $V_{\text {in }}$ and hence enhancing linearity, only when the following relationship is satisfied,

$$
\frac{\left(V_{G S 1}+V_{G S 9}-V_{G S 10}-V_{G S 2}\right)}{R_{t}}=\frac{\left(V_{G S 3}+V_{G S 7}-V_{G S 8}-V_{G S 4}\right)}{R_{b}}
$$

For Equation 5.3.6 to be satisfied, the following Equations 5.3.7 and 5.3.8 must be true:

$$
\begin{aligned}
& \left(V_{G S 3}-V_{G S 1}\right)+\left(V_{G S 10}-V_{G S 8}\right)=0 \\
& \left(V_{G S 2}-V_{G S 4}\right)+\left(V_{G S 7}-V_{G S 9}\right)=0
\end{aligned}
$$

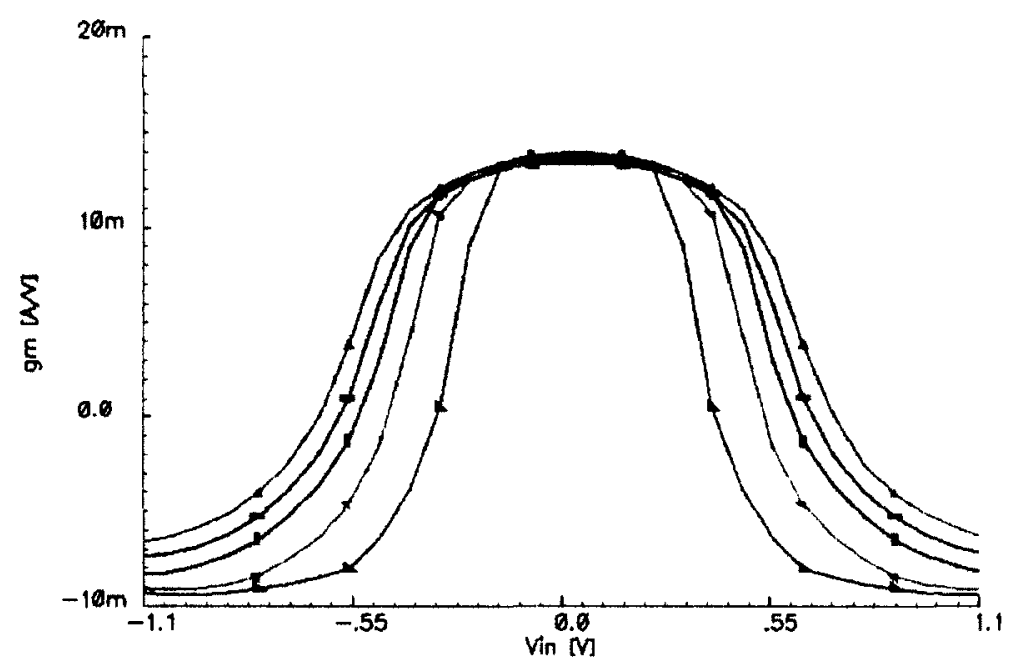

Figure 5.5: CMOS cross-coupled compensator tunable gm flatness versus input signal.

Equations 5.3.7 and 5.3.8 are met by adjusting both bias current sources Ibias $_{t}$ and Ibias $_{b}$. Having the device sizes with unequal dimensions, equation 5.3.7 and equation 5.3.8 can be satisfied by tuning both bias current source Ibias $_{t}$ and Ibias . Transistors with smaller device size (W/L) mean that their equivalent gate-source voltage $V_{g s}$ changes 
faster. Transistors with larger device size (W/L) have their gate-source voltage $V_{g s}$ change slower. When both bias current source Ibias $t$ and Ibias $b$ are adjusted, the cross-coupled compensator $g_{m}$ is tuned achieving higher linearity. Hence the total differential output current $\Delta I_{\text {out }}$ achieves a linear relationship with the input differential voltage $V_{\text {in }}$.

To ensure circuit stability the device dimensions were optimized dimension of $\left[M_{1}, M_{2}\right]$ width (W/L) equal to $96 \mu \mathrm{m}$ and $\left[M_{3}, M_{4}\right](\mathrm{W} / \mathrm{L})$ equal to $72 \mu \mathrm{m}$ and $\left[M_{7}, M_{8}\right](\mathrm{W} / \mathrm{L})$ equal to $32 \mu \mathrm{m}$ and $\left[M_{9}, M_{10}\right](\mathrm{W} / \mathrm{L})$ equal to $20 \mu \mathrm{m}$ with all devices having $\mathrm{L}$ minimum channel length of $120 \mathrm{~nm}$. Simulation results show that the proposed linearized transconductor achieves a tunable 50 percent increase in the device transconductor parameter gm flatness over wide differential input signal as shown in Figure 5.5 and a wide second derivative device transconductor parameter $\mathrm{gm}$ " nulling over $500 \mathrm{mV}$ as shown in Figure 5.6. The proposed transconductor [100] maintains a high input voltage swing range due to the aid of the second translinear loop $\left(M_{3}-M_{4}\right)$.

The distributed 3-stage CMOS cross-coupled compensator transconductor structure is formed through adopting a low-pass artificial transmission line into a bidirectional DA as shown in Figure 5.4. Each CMOS cross-coupled compensator transconductor stage is separated with series on-chip spiral inductors used to extend the operation frequency range. The currents from each stage are combined at the output terminal and therefore the third-order intermodulation IM3 distortion reduction is effective over a broad bandwidth.

\subsection{Effect of Nonlinear Drain Capacitance on DA Lin- earization Bandwidth}

Another source of nonlinearity in CMOS bidirectional DAs is the transmission line nonlinear drain capacitance $C_{d}$. A nonlinear drain current is induced flowing out of the drain of an NMOS transistor. This nonlinearity can be reduced by introducing a parallel inverse nonlinearity at the transmission drain line at the output of the CMOS distributed structure to compensate for the drain capacitance of the active element as depicted in Figure 5.4 (b). The PMOS varactor-based active PMOS nonlinear capacitance compensator tunes the nonlinear drain capacitance providing IM3 distortion cancellation at various varactor voltages. The transmission-line capacitance is part of the filter structure whose bandwidth 


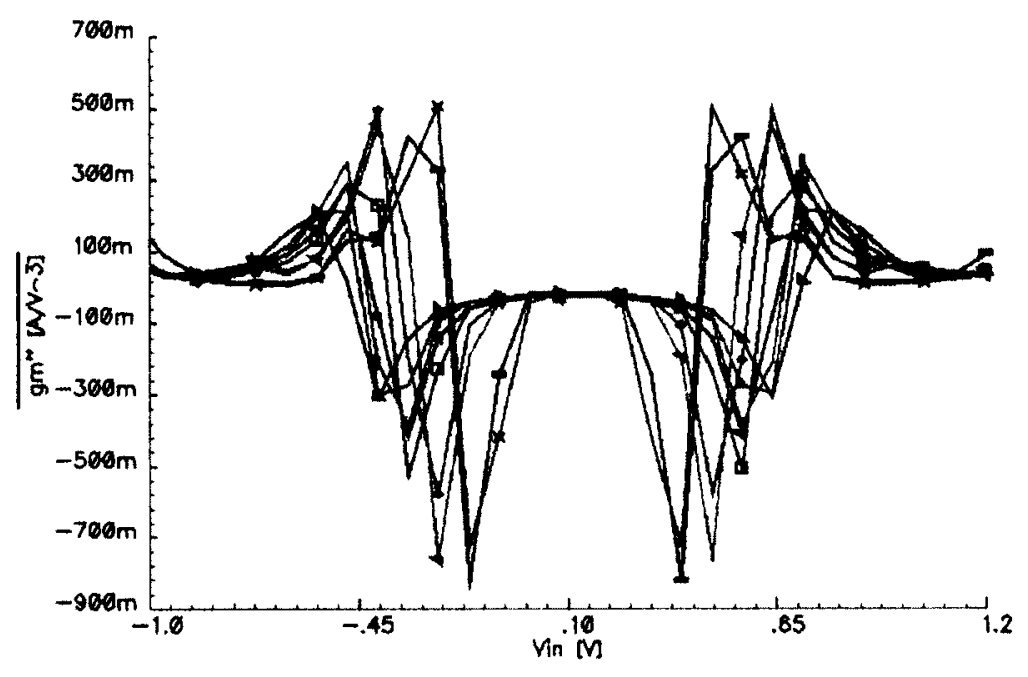

Figure 5.6: Simulation result of CMOS cross-coupled compensator wide tunable gm" nulling versus input signal.

is determined by the amount of transmission-line inductance and capacitance of the filter section. The transmission-line cut-off frequency $f_{c}$ is widened when the transmission-line capacitance is reduced ( [85], [31])

$$
f_{c}=\frac{1}{\pi \sqrt{L C}}
$$

The nonlinear element drain capacitance $C_{d}$ is a function of $v_{d s}$ and can be expressed by power series with coefficients $C_{d 0}, C_{d 1}$ and $C_{d 2}$

$$
C_{d}=C_{d 0}+C_{d 1} V_{d s}+C_{d 2} V_{d s}^{2}
$$

The nonlinear element $C_{v a r}$ is a function of $v_{d s}$ and can be expressed by power series with coefficients $C_{v a r 0}, C_{\text {var1 }}$ and $C_{\text {var2 }}$

$$
C_{v a r}=C_{v a r 0}+C_{v a r 1} v_{d s}+C_{v a r 2} v_{d s}^{2}
$$

Adding both nonlinear capacitances in Equation 5.4.2 and the inverse nonlinear capacitance Equation 5.4.3 compensate the total amount of nonlinear drain parasitic capacitance by tuning the varactor PMOS active nonlinear compensator. 


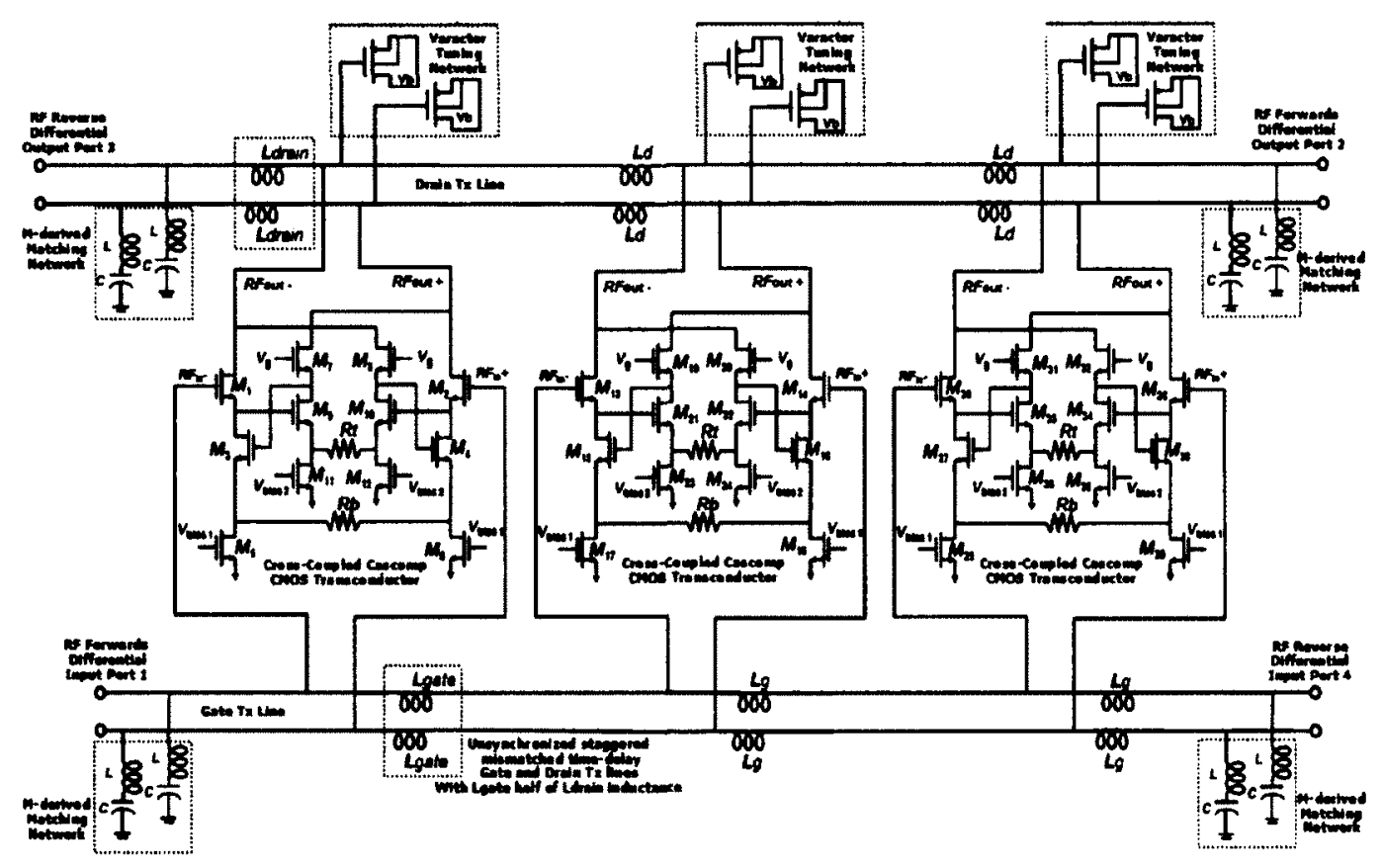

Figure 5.7: The proposed schematic of the three-stage bidirectional distributed amplifier with CMOS cross-coupled compensator transconductor gain cells coupling the staggered drain and gate transmission-lines with nonlinear drain capacitance compensator.

$$
C_{\text {compensator }}=C_{d}+C_{v a r}
$$

The varactor-based active PMOS distortion linearizer compensates for drain capacitance nonlinearities. Figure 5.4 (b) illustrates that the linearity of the parasitic drain capacitance in the CMOS distributed structure can be adjusted by adding an inverse parallel varactor PMOS device to reduce the nonlinearity of the overall DA drain capacitance hence achieving highly-linear performance [31]. Figure 5.7 shows the proposed schematic of the linearized CMOS bidirectional DA.

The bidirectional properties of a conventional distributed amplifier have been compared to that of an ideal duplexer/circulator ( [19], [21]). The four-port distributed amplifier is inherently bidirectional because of the symmetry in its architecture. It can be excited either from port (1) or port (4). Thus it can be driven from both ends of the gate lines 
simultaneously. In Figure 5.7, the proposed CMOS bidirectional distributed amplifier based tunable active duplexer has port (1) and port (4) as input ports and port (2) and port (3) as output ports. Signal power fed into port (1) emerges from port (TX2) as depicted in Figure 5.7 and isolation is provided between port (1) and port (3).

We define the gain of the distributed amplifier as the ratio of the forward output power at port (2) to the input power at port (1) and the directivity for the distributed amplifier as the ratio of the reverse output power at port (3) to the forward output power at port (2). In $\mathrm{dB}$ this is given as ([19], [20], [74], [108])

$$
\begin{gathered}
G=10 \log \left[P_{\text {out }}^{+} / P_{\text {in }}^{+}\right]=20 \log \left[S_{21}\right] \\
D=-10 \log \left[P_{\text {out }}^{-} / P_{\text {out }}^{+}\right]=-20 \log \left[S_{31} / S_{21}\right]
\end{gathered}
$$

The distributed amplifier based active duplexer directivity can be improved through the tuning of $S_{31}$ isolation over broad bandwidth. In the case of ideal distributed amplifier with no losses, $S_{21}$ and $S_{31}$ can be defined as ([19], [20])

$$
\begin{aligned}
& S_{21}=-\frac{1}{2} Z_{\pi} e^{-j \beta N}\left[\sum_{i=0}^{N} g_{m_{i}}\right] \\
& S_{31}=-\frac{1}{2} Z_{\pi}\left[\sum_{i=0}^{N} g_{m_{i}} e^{-j \beta 2 i}\right]
\end{aligned}
$$

where $\beta$ is the phase shift per $\pi$-section along the lines and $\mathrm{n}$ represents the number of devices in the amplifier. $Z_{\pi}$ is the $\pi$-section image impedance of the drain line and $g_{m_{i}}$ is the transconductance of the $i_{t h}$ device.

From equations 5.4.5 and 5.4.7 the gain of the amplifier can be expressed as ( [74], [108])

$$
G=20 \log \left|\frac{1}{2} Z_{\pi} e^{-j \beta N}\left[\sum_{i=0}^{N} g m_{i}\right]\right| .
$$

From equations 5.4.6 and 5.4.8 the directivity of the amplifier is 


$$
D=20 \log \frac{\left|-\frac{1}{2} Z_{\pi} e^{-j \beta N}\left[\sum_{i=0}^{N} g_{m_{i}}\right]\right|}{\left|-\frac{1}{2} Z_{\pi}\left[\sum_{i=0}^{N} g_{m_{i}} e^{-j \beta 2 i}\right]\right|}
$$

From equation 5.4 .5 the directivity can be expressed in the form ( [19], [108])

$$
D=G-20 \log \left|-\frac{1}{2} Z_{\pi}\left[-20 \log \sum_{i=0}^{N} g_{m_{i}} e^{-j \beta 2 i}\right]\right|
$$

The power gain increases with the increase of number of DA gain cell stages. Amplification gain stages are connected so that output currents are combined in an additive manner at the output terminal. The gain can be increased by introducing more sections. As the RF input signal travels down the gate transmission-line, each FET transistor is excited by the traveling power wave and transfers the signals to the drain line through its transconductance. The advantages of a distributed amplifier topology are its wide bandwidth, flat gain and compact size circuit size. However, in the presence of attenuation, the gate wave signal decays as it propagates down the line. Hence, there will be a point at which the gain added by an additional device will not overcome the losses induced by the extra section in the gate and drain lines. The reason for this is that the devices added are not driven sufficiently to overcome the losses in the drain line cause of high signal attenuation.

Figure 5.8 also shows the simulated return loss $S_{11}, S_{22}$ and $S_{21}$ differential power gain of the three-stage bidirectional distributed amplifier peaks at $6 \mathrm{~dB}$ and then rolls off to a unity gain bandwidth of $11.5 \mathrm{GHz}$. The simulated input and output matching $S_{11}$ and $S_{22}$ are both below $-10 \mathrm{~dB}$ indicating less of the power transferred would be reflected. The simulated isolation $S_{12}$ performance is better than $-26 \mathrm{~dB}$.

Simulated IIP3 before and after linearization results for linearized CMOS bidirectional distributed amplifier are shown in Figure 5.9. A $10 \mathrm{~dB}$ IIP3 improvement can be seen from Figure 5.9.

Intermodulation distortion (IMD) nonlinearity appears as a result of applying two tones to the distributed bidirectional amplifier at $1 \mathrm{GHz}$ with IP3 of $6 \mathrm{dBm}$. Simulated IM3 before linearization is shown in Figure 5.10.

The degree of intermodulation distortion (IMD) nonlinearity appears less with IP3 


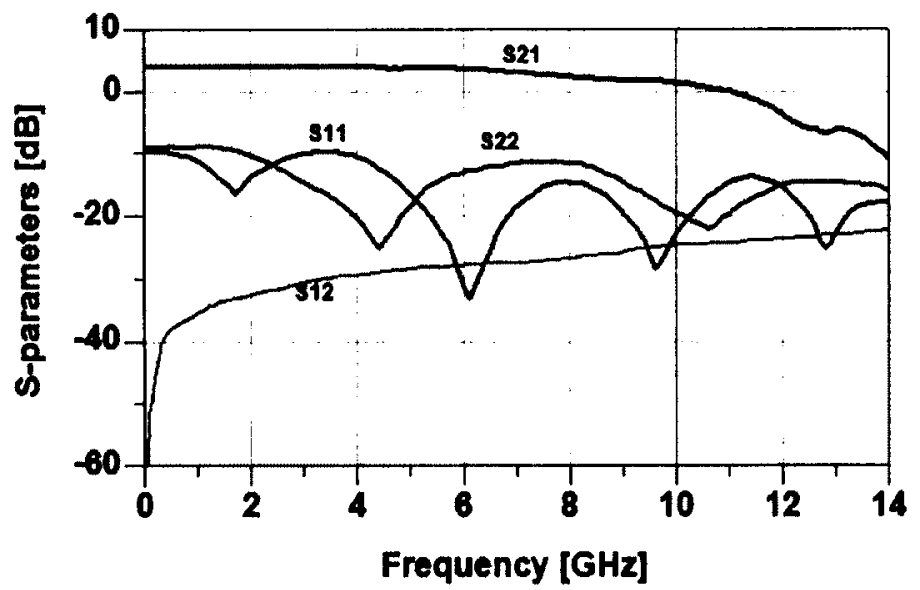

Figure 5.8: Simulated differential $S_{21}$ power gain, $S_{11}$ and $S_{22}$ return losses and $S_{12}$ isolation for the three-stage fully-differential linearized CMOS bidirectional distributed amplifier.

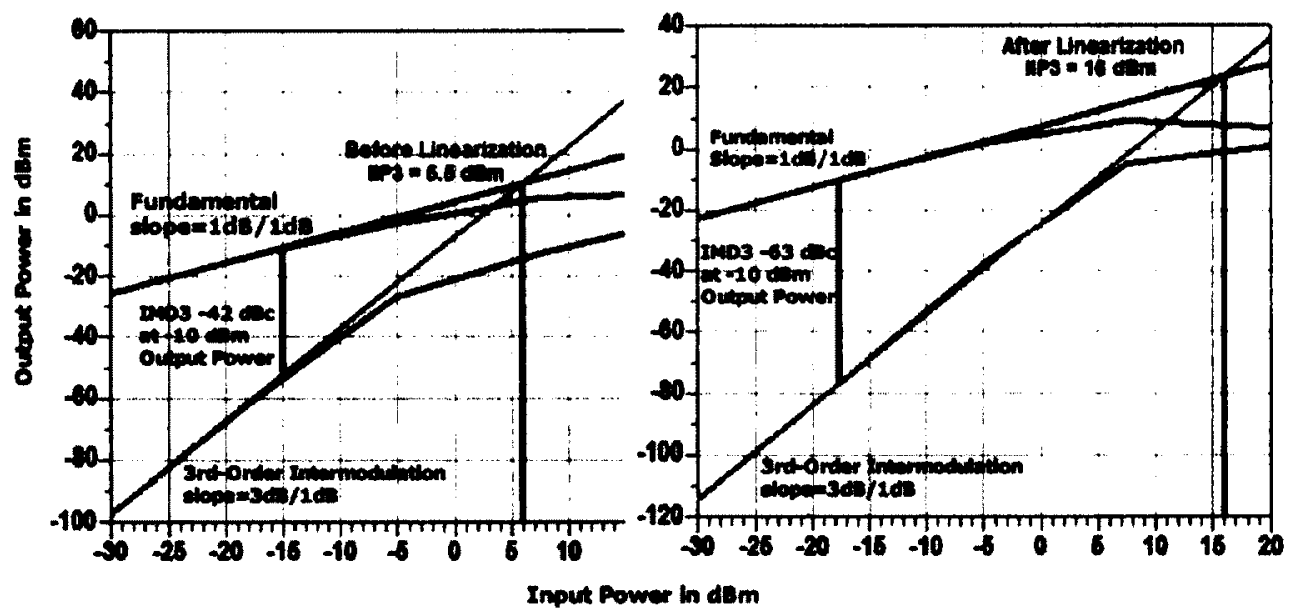

Figure 5.9: Simulated IIP3 before and after linearization for linearized CMOS bidirectional distributed amplifier. 


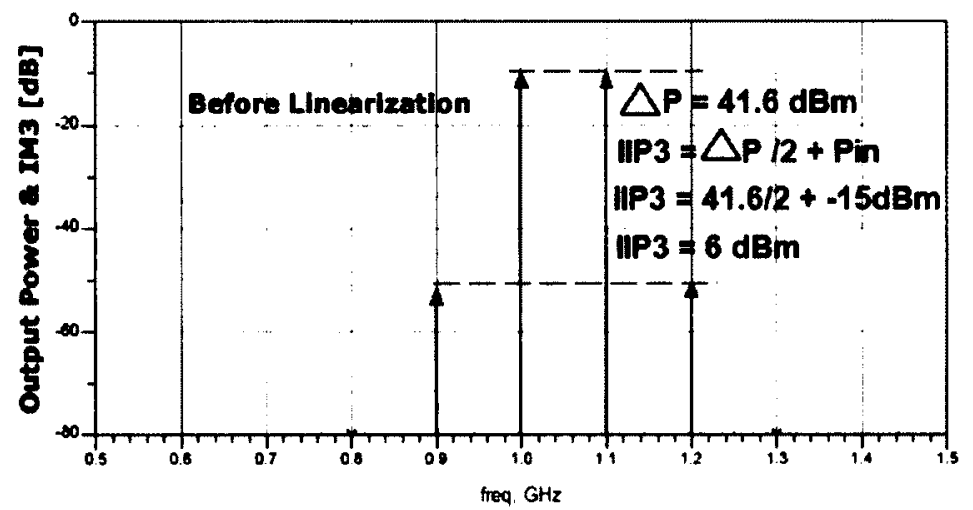

Figure 5.10: Simulated IM3 before linearization at $1 \mathrm{GHz}$ for linearized CMOS bidirectional distributed amplifier.

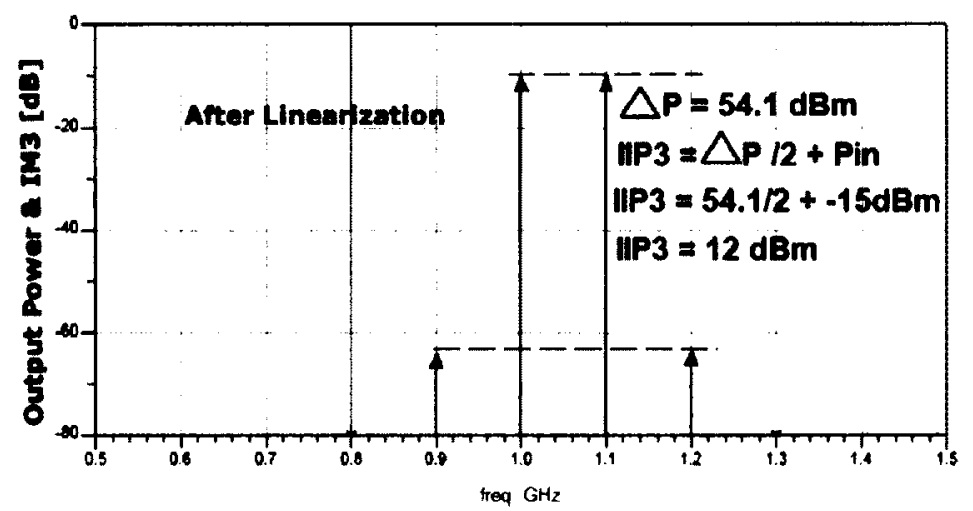

Figure 5.11: Simulated IM3 after linearization at $1 \mathrm{GHz}$ for linearized CMOS bidirectional distributed amplifier. 
improving to $12 \mathrm{dBm}$. Simulated IM3 after linearization at $1 \mathrm{GHz}$ for linearized CMOS bidirectional distributed amplifier is shown in Figure 5.11.

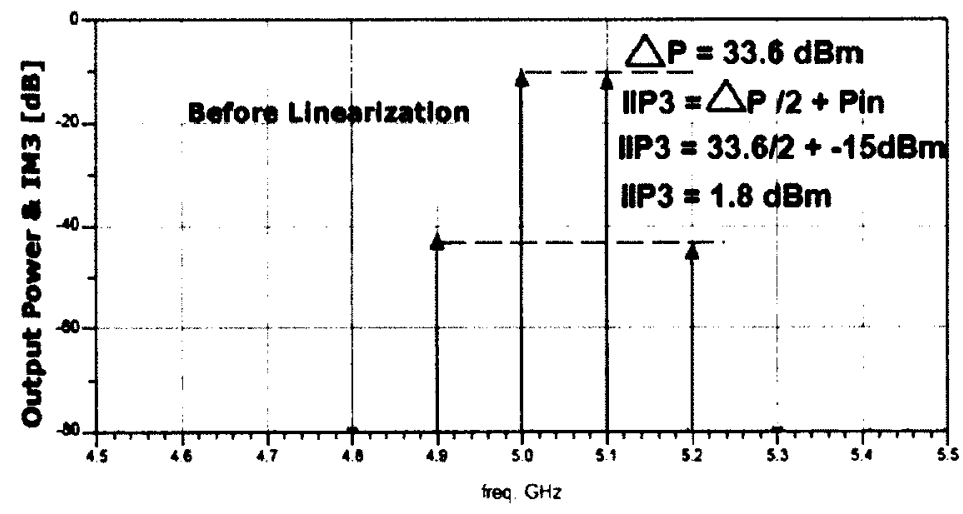

Figure 5.12: Simulated IM3 before linearization at 5GHz for linearized CMOS bidirectional distributed amplifier.

Intermodulation distortion (IMD) nonlinearity appears as a result of applying two tones to the distributed bidirectional amplifier at $5 \mathrm{GHz}$ with IP3 of $1.8 \mathrm{dBm}$. Simulated IM3 before linearization at $5 \mathrm{GHz}$ for linearized CMOS bidirectional distributed amplifier is shown in Figure 5.12.

The degree of intermodulation distortion (IMD) nonlinearity appears less with IP3 improving to $10.3 \mathrm{dBm}$. Simulated IM3 after linearization at $5 \mathrm{GHz}$ linearized CMOS bidirectional distributed amplifier is shown in Figure 5.13.

Simulated fundamental and third-order intermodulation distortion for one-stage CMOS cross-coupled cascomp transconductor is shown in Figure 5.14.

Simulated stability factor $K_{f}$ and $B_{1 f}$ characterizing the linearized bidirectional distributed amplifier stability is shown in Figure 5.15. 


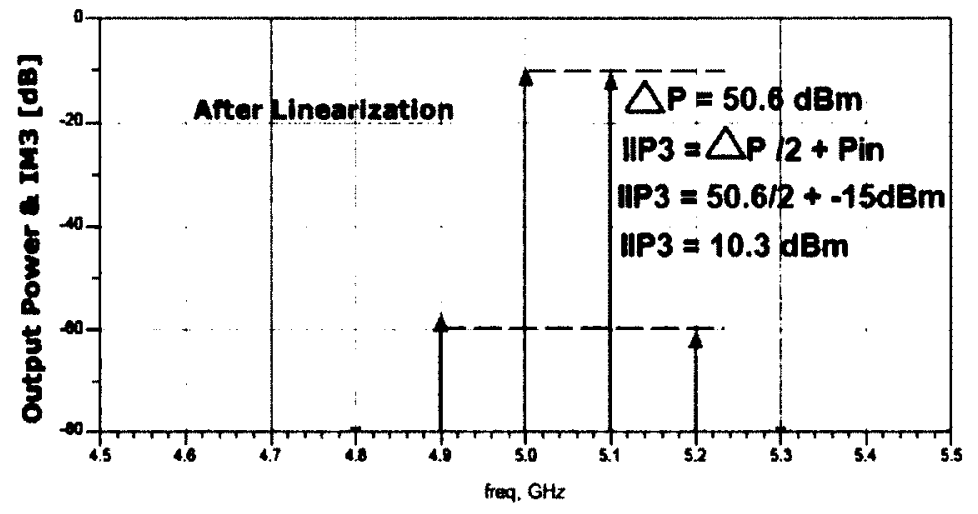

Figure 5.13: Simulated IM3 after linearization at $5 \mathrm{GHz}$ for linearized CMOS bidirectional distributed amplifier.

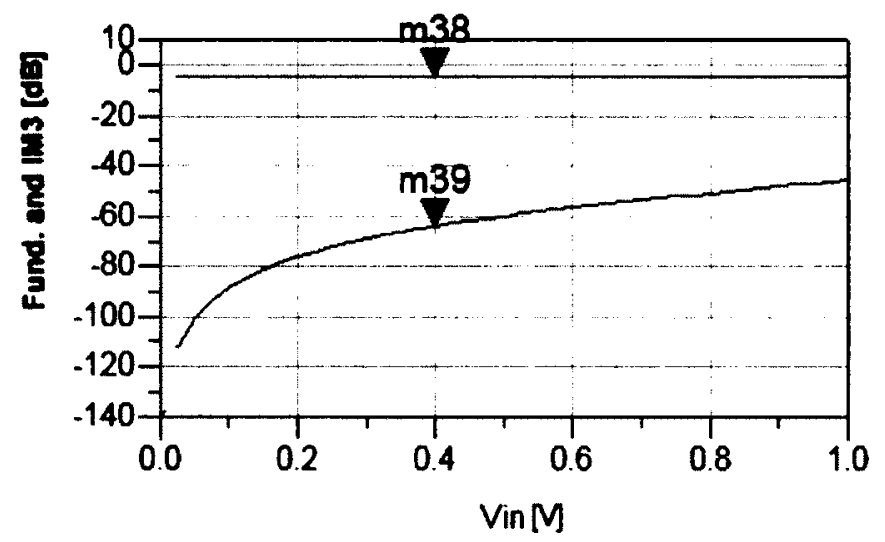

Figure 5.14: Simulated fundamental and third-order intermodulation distortion for onestage CMOS cross-coupled cascomp transconductor. 


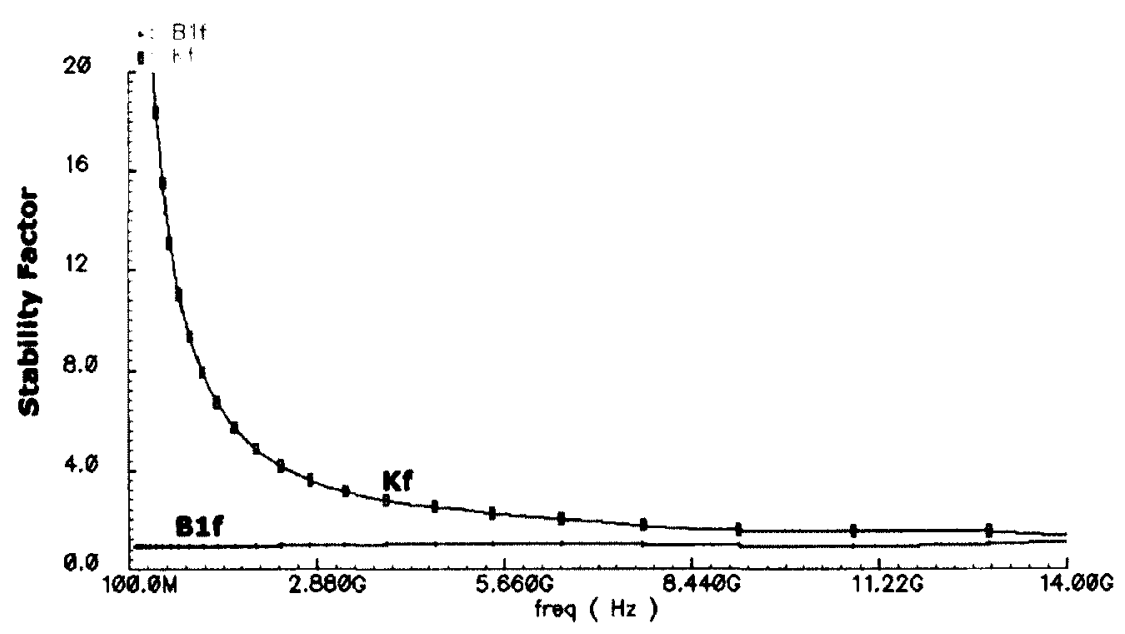

Figure 5.15: Simulated stability factor $K_{f}$ and $B_{1 f}$ characterizing linearized bidirectional distributed amplifier stability.

\subsection{Transmission-Lines Multi-level Inductor Model- ing in in Transmission-Lines for Silicon Chip Area Reduction}

The CMOS linearized distributed amplifier has been optimized using multi-level lumped inductor elements in order to obtain a circuit that is silicon area efficient. Further reduction in chip area is achieved using multi-level inductors ([109], [33]). These multi-level structures benefit from strong mutual coupling between vertically adjacent metal layers, and can generate the same inductance in less area as compared with planar inductors as shown in Figure 5.16.

Two-port interconnect lumped model for multi-level inductor broadband equivalent circuit is shown in Figure 5.19. Equivalent circuit model parameters are extracted from the frequency dependent quasi-static solution of the physical substrate structure and consists of ideal R, C and L components. Substrate loss for interconnect is modeled by the resistor $R_{s u b}$ and capacitor network that consists of $C_{o x}$ and $C_{s u b}$ representing the oxide layer parasitic capacitances between the conductors and the bulk substrate ( $[76],[31],[110])$. 


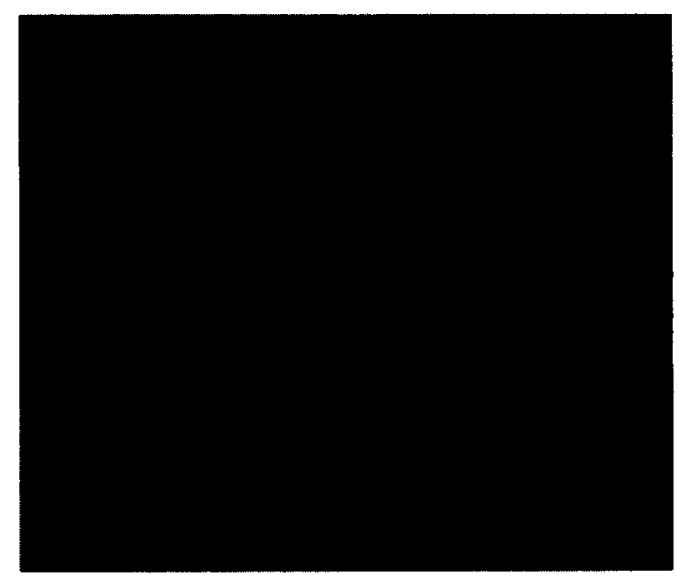

Figure 5.16: HFSS linearized CMOS bidirectional distributed amplifier transmission-lines multi-level inductor modeling RF CMOS $0.13 \mu \mathrm{m}$. The HFSS modeled inductor has an outer diameter of $91 \mu \mathrm{m}$ and spacing of $5 \mu \mathrm{m}$ with width of $10 \mu \mathrm{m}$.

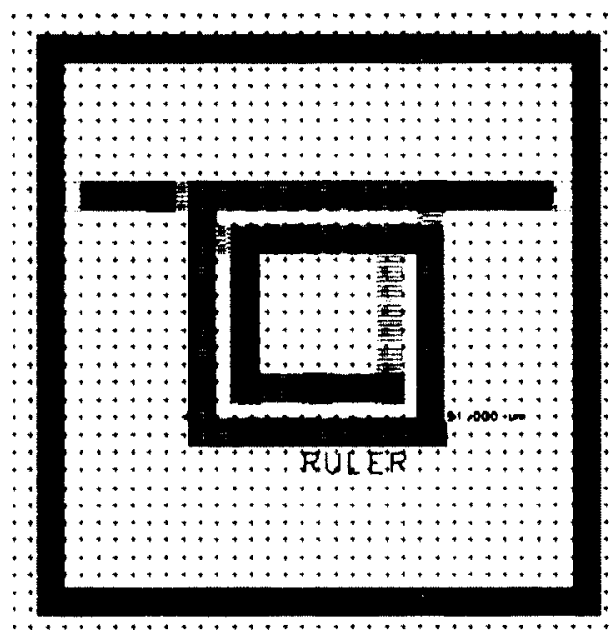

Figure 5.17: ADS linearized CMOS bidirectional distributed amplifier transmission-lines multi-level inductor modeling RF CMOS $0.13 \mu \mathrm{m}$. 


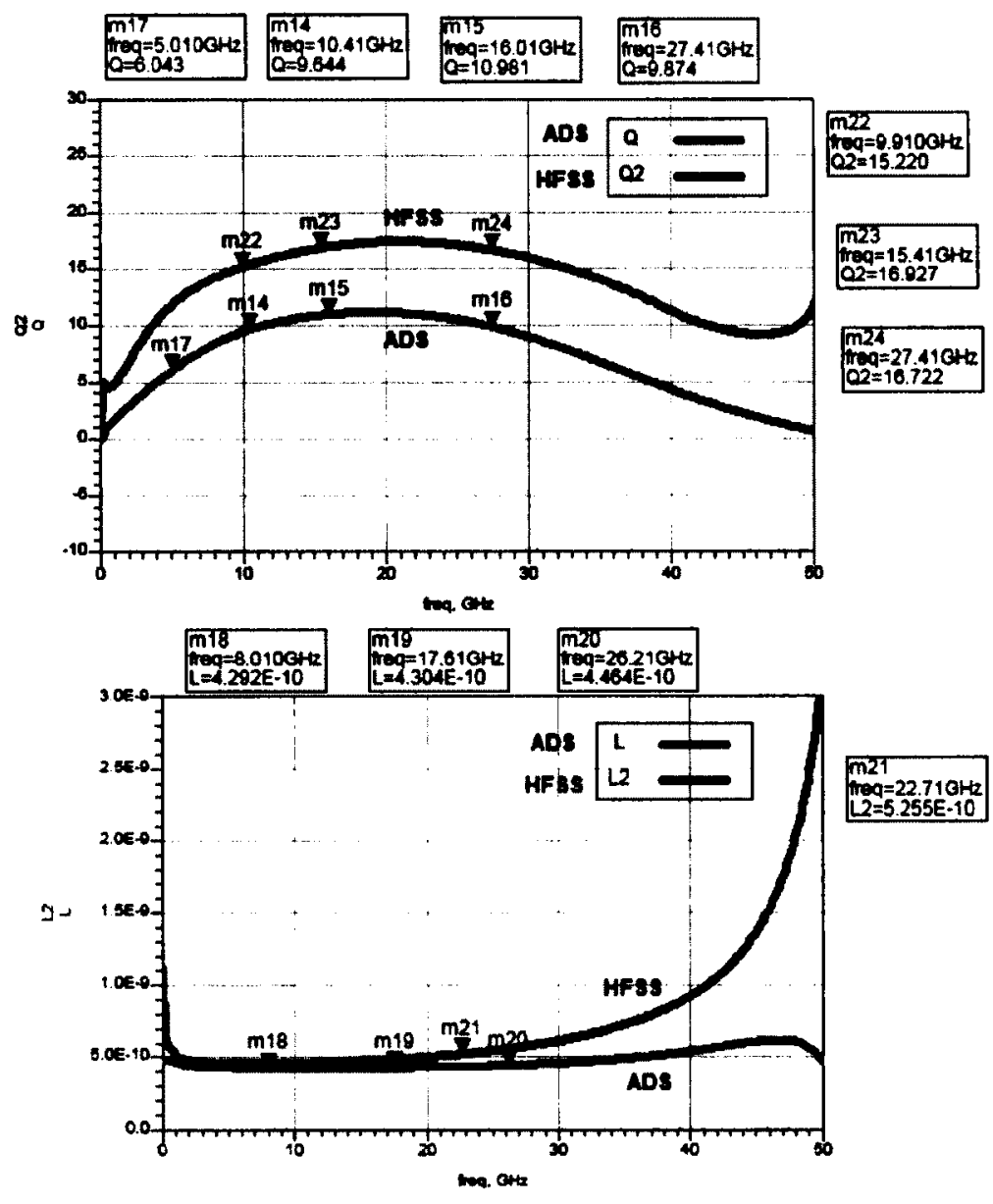

Figure 5.18: HFSS and ADS linearized CMOS bidirectional distributed amplifier gate transmission-lines multi-level inductor modeling $Q$ and $L$ Modeling RF CMOS $0.13 \mu \mathrm{m}$. The ADS EM calculations were performed using the methods of moments where as in HFSS the 3D full-wave EM calculations were performed using the finite element method. 
Accurate modeling at microwave frequencies requires electromagnetic simulations using ADS Momentum or HFSS EM engines as shown in Figure 5.18. A $0.13 \mu \mathrm{m}$ CMOS silicon substrate was constructed in both HFSS and ADS Momentum, based on the available data from process foundry as shown in Figure Figure 5.17. The IBM $0.13 \mu \mathrm{m}$ CMOS process offers three thick RF metal layers suitable for high-Q inductors and the top metal layer (MA) is composed of aluminum. In order to reduce the loss and improve the quality factor $(\mathrm{Q})$, the metallization layer with the lowest loss is chosen for the design. Inductor $Q$ as shown in Figure Figure 5.18 is frequency dependent. It is clear that inductor $Q$ is strongly affected by the metal thickness (which related to metal loss) and substrate resistivity.

Stacked inductors implemented in two or three metal layers were designed with inductance values $900 \mathrm{pH}$ and $450 \mathrm{pH}$. The gate transmission-line $450 \mathrm{pH}$ inductor has an outer diameters of $70 \mu \mathrm{m}$ and spacing of $5 \mu \mathrm{m}$ and width of $9 \mu \mathrm{m}$ and number of turns $\mathrm{n}$ of 1.5 . The drain transmission-line $900 \mathrm{pH}$ inductor has an outer diameter of $91 \mu \mathrm{m}$ and spacing of $5 \mu \mathrm{m}$ and width of $10 \mu \mathrm{m}$ and number of turns $\mathrm{n}$ of 1.75 . Both stacked inductors were implemented on MA and E1 top metal layers which corresponds to the eight and seventh top metal layers. The gate transmission-line m-derived $280 \mathrm{pH}$ inductor has an outer diameter of $59 \mu \mathrm{m}$ and spacing of $5 \mu \mathrm{m}$ and width of $9 \mu \mathrm{m}$ and number of turns $\mathrm{n}$ of 1.5. The drain transmission-line m-derived $230 \mathrm{pH}$ inductor has an outer diameter of $55 \mu \mathrm{m}$ and spacing of $5 \mu \mathrm{m}$ and width of $9 \mu \mathrm{m}$ and number of turns $\mathrm{n}$ of 1.5 .

As with planar inductors, reducing area over substrate is paramount in increasing the resonance frequency (SRF) of stacked inductors ( [76], [31], [15]). The nearly 50 percent reduction in total area with more metal layers yields higher SRF, even though the bottom metal layer is slightly closer to the substrate. EM simulations in HFSS and ADS were performed on the gate and drain transmission-lines multi-level inductors. The EM simulation results of $Q$ and inductance $L$ in RF CMOS $0.13 \mu \mathrm{m}$ are shown in Figure 5.18. The ADS EM calculations were performed using the methods of moments where as in HFSS the 3D full-wave EM calculations were performed using the finite element method. The EM simulations results for both calculations are shown in Figure 5.18.

CMOS processes have low resistive substrates which cause significant losses which makes design of high $\mathrm{Q}$ inductors challenging. At low frequencies, metal losses are mainly determined by the sheet resistance of the process layers used to create the device. However, at 


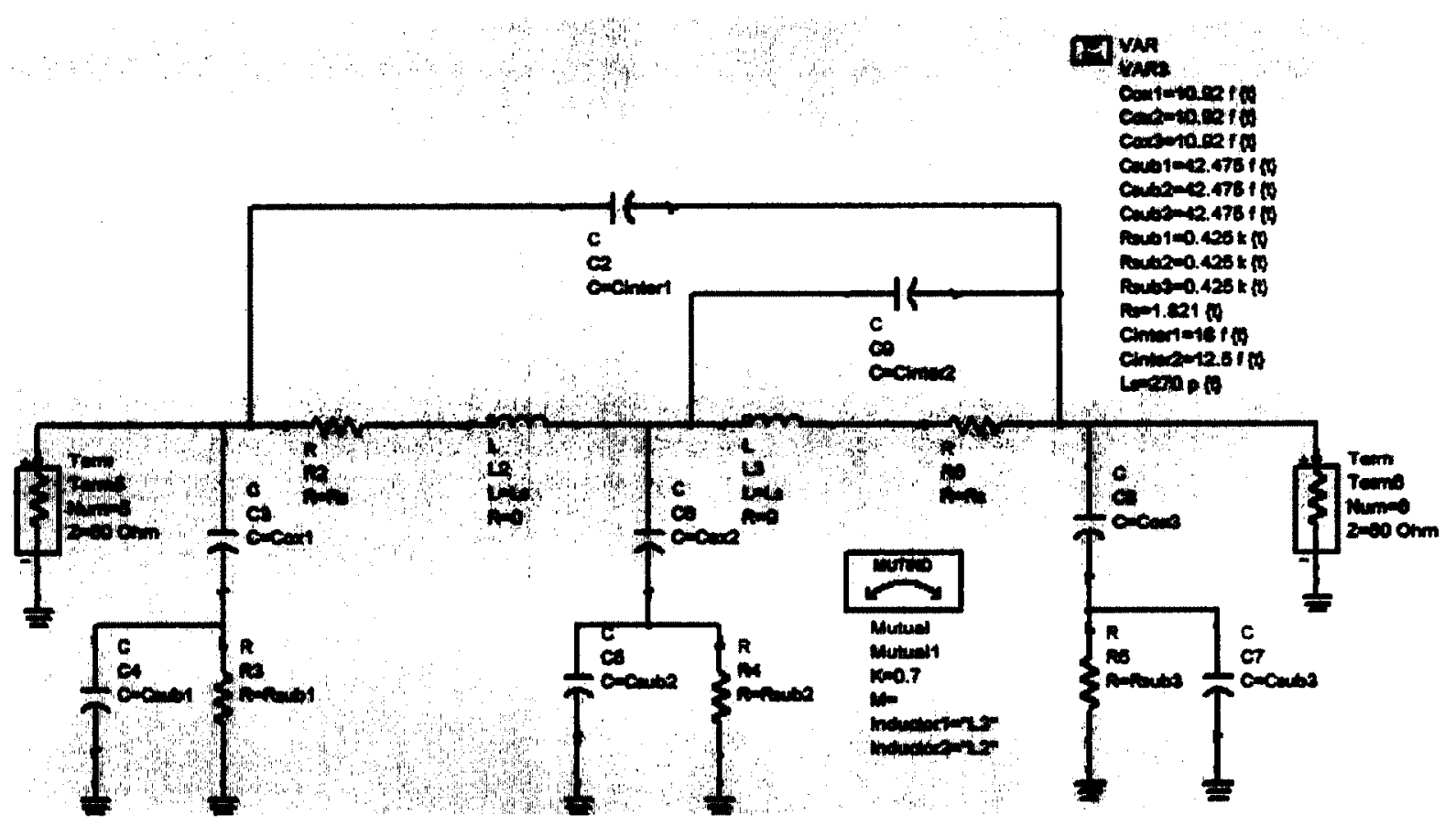

Figure 5.19: ADS transmission-lines multi-level inductor broadband equivalent circuit model in RF CMOS $0.13 \mu \mathrm{m}$. The multi-level inductor broadband equivalent circuit component values were determined by curve-fitting with the EM HFSS and ADS simulated results. 

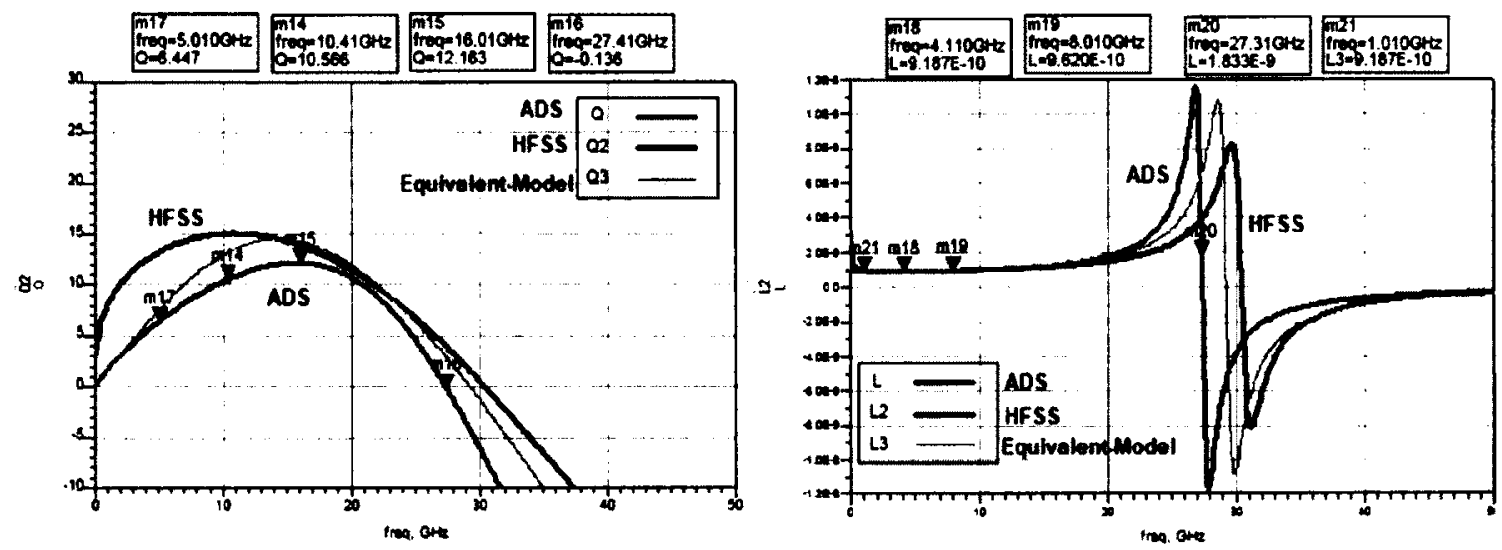

Figure 5.20: HFSS and ADS drain transmission-line multi-level inductor Q and L modeling for RF CMOS $0.13 \mu \mathrm{m}$.

high frequencies, skin effect, proximity effects and current crowding have a major impact on the loss mechanism ( [76], [31], [27], [29]). The skin effect drives the ac current toward the surface of the conductor. Skin effect increases the ac resistance of the conductor leading to lower Q ( [111], [112], [113], [114]).

Inductor performance is strongly affected by the metal loss and substrate loss. Increasing metal thickness to reduce metal loss could significantly improve inductor $Q$ for RF applications. A CMOS inductor's performance is also affected by the oxide thickness beneath the inductor and its lateral dimensions, such as metal strip width, spacing, and outer diameter ( [66], [115], [116]).

Proximity effect metal loss degrades the performance of on-chip inductor at high frequency due to the influence of the magnetic field created by a nearby conductor and thereby increasing the effective series resistance. The two-level inductor has a peak of approximately 15 and it peaks at $25 \mathrm{GHz}$ with a self-resonance frequency of $50 \mathrm{GHz}$. Effective inductance of $450 \mathrm{pH}$ and quality factor $\mathrm{Q}$ of 13 for the stacked inductor are shown in Figure 5.18. The multi-level inductor broadband equivalent circuit model in RF CMOS $0.13 \mu \mathrm{m}$ is shown in Figure 5.19. The multi-level inductor broadband equivalent circuit component values were determined by curve-fitting with the EM HFSS and ADS simulated results. The drain transmission-line multi-level inductor quality factor $\mathrm{Q}$ and 
inductance $\mathrm{L}$ modeling for RF CMOS $0.13 \mu \mathrm{m}$ with inductance of $910 \mathrm{pH}$ and $\mathrm{Q}$ of 15 is shown in Figure 5.20.

\subsubsection{Varactor-tuned LC Networks}

The proposed linearized CMOS bidirectional distributed amplifier achieves linearization on two parameters, transconductance using the proposed CMOS cross-coupled cascomp and drain nonlinear parasitic capacitance compensation using the MOS varactors. The MOS capacitor is gate voltage dependent, so when using a MOS capacitor depending on biasing condition, the capacitance will be small, lossy, and highly nonlinear.

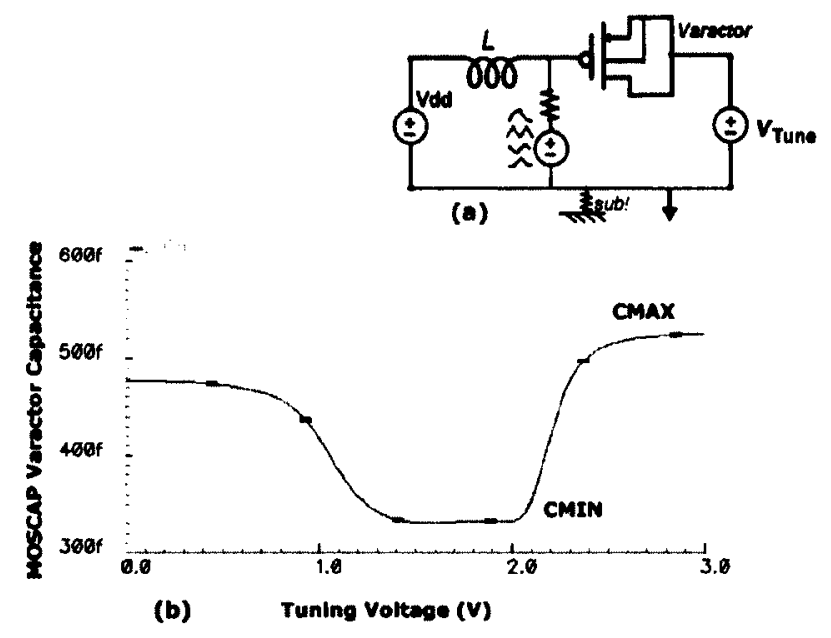

Figure 5.21: (a) MOSCAP varactor test setup schematic with source and drain connected together to generate a highly non-linear capacitance-voltage curve behavior (b) C-V characteristic of the minimum and maximum MOSCAP varactor capacitance.

The test schematic showing the testbench when the MOSCAP varactor (variable reactors or voltage controlled capacitors) with source and drain connected together to act as an inversion mode capacitance by varying the voltage is shown in Figure 5.21 with the MOSCAP minimum and maximum value of the varactor capacitance simulation results. Simulated Performance of the linearized CMOS bidirectional distributed amplifier is shown 
in Table 5.1. The power gain $S_{21}$ of the three-stage bidirectional distributed amplifier peaks at $6 \mathrm{~dB}$ and then rolls off to a unity gain bandwidth of $11.5 \mathrm{GHz}$. The simulated input and output matching $S_{11}$ and $S_{22}$ are both below -10 dB indicating less of the power transferred would be reflected. The simulated isolation $S_{12}$ performance is better than $-26 \mathrm{~dB}$.

Table 5.1: Simulated Performance of the linearized CMOS bidirectional distributed amplifier

\begin{tabular}{|c||c|}
\hline Technology & RF CMOS $0.13 \mu \mathrm{m}$ \\
\hline Unity Gain Bandwidth & $11.5 \mathrm{GHz}$ \\
\hline$S_{21}$ Peak Power Gain & $6 \mathrm{~dB}$ \\
\hline Linearized IIP3 & $>10 \mathrm{~dB}$ \\
\hline$S_{12}$ Isolation & $<-26 \mathrm{~dB}$ \\
\hline Silicon area & $1.887 \mathrm{~mm} \mathrm{X} 0.795 \mathrm{~mm}$ \\
\hline
\end{tabular}

\subsection{Chapter Summary}

In this chapter, a fully-integrated fully-differential linearized CMOS distributed bidirectional amplifier that achieves large IMD3 distortion reduction over broadband frequency range for both RF paths was demonstrated. The drain and gate transmission-lines were stagger-compensated. Reducing the DA IM3 distortion by mismatching the gate and drain LC delay-line ladders. A CMOS cross-coupled compensator transconductor is proposed to enhance the linearity of the DA gain cell with a varactor-based active post nonlinear drain capacitance compensator for wider linearization bandwidth. 


\section{Chapter 6}

\section{Linearized CMOS Distributed Bidirectional Amplifier Silicon Chip Implementation}

\subsection{Introduction}

This chapter presents several practical layout guidelines of the fully-integrated fully-differential linearized distributed bidirectional amplifier implemented in IBM CMOS RF $0.13 \mu \mathrm{m}$ silicon process. The total chip silicon area is $1.5 \mathrm{~mm}^{2}$ including testing pads. The circuit elements forming the linearized CMOS bidirectional distributed amplifier are discussed in terms of their physical arrangement and layout.

\subsection{Linearized CMOS Bidirectional Distributed Am- plifier High Frequency Layout Considerations}

CMOS technologies offer the capability of integrating both baseband and RF front-end transceiver components on a single chip allowing low cost implementation [117]. However, CMOS has inherent technology limitations such as low current drive, lossy substrate, low breakdown voltage and poor transconductance ( [34], [33]). These technology drawbacks have negative impact on implementing fully-integrated power amplifier using CMOS process. For this reason, a fully differential topology has been adopted since differential power amplifier have several advantages over single-ended ones. For instance, the voltage doubling effect lowers the burden of low breakdown voltage limit. Also, the virtual ground 
of source prevents gain reduction from source inductance degeneration and stability can be easily achieved [36].

The design of the proposed linearized bidirectional DA has been fully-integrated in $0.13 \mu \mathrm{m}$ RF CMOS technology. Proper layout techniques are used such as maintaining device matching and use of symmetry in circuit layout design results in process variation reduction [?]. Fully-differential circuit topology used in the proposed design will reduce susceptibility to supply noise, supply bounce and will reject common-mode noise. The use of balanced modular layout design results in balanced current distribution and supply routing ( [34], [33]). Differential circuits also impose upon layout to be symmetrical which will reduce mismatches in threshold voltage and current gain of the circuit devices. With interdigitating wide transistors, with multi-gate finger layout, the gate resistance of the polysilicon becomes smaller. The chip area is minimized by the use of stacked inductors which will reduce the fluctuation of process variation. Grounded "guard ring" are added surrounding sensitive active devices to improve device isolation and acts as an effective shield to noise and crosstalk. ESD were added to the gates in order to protect the chip from antenna effects [36].

Technology process parameters such as substrate resistivity, number of metal layers, distance between metal layers, and metal layer thickness are all set by $0.13 \mu \mathrm{m}$ CMOS technology [118]. The $0.13 \mu \mathrm{m}$ CMOS top most metal layer has lower sheet resistance and suffers less loss. The top metal layer has the largest thickness which helps in improving component quality factor $Q$ and it is commonly utilized for routing critical high frequency signals. CMOS $0.13 \mu \mathrm{m}$ technology offers eight metal layer (M1-M8) [118] including three thin metal (M1, M2, and M3) layers, two thick metal (MQ and MG) layers and three RF (LY, E1, and MA) layers. Modeling the substrate definition includes the number of layers, position of each layer and composition of each layer.

The drain transmission line signal conductor dimensions are made wide in order to reduce the resistivity on the drain transmission lines which greatly affects the high-frequency performance of the device. Gate tie-downs where added to long metal traces in order to cancel antenna effect preventing the collection of charges that may destroy the device gates. ESD double diode protection were added to $\mathrm{I} / \mathrm{O}$ pads and multiple contacts are placed where needed to ensure good connections ( [34], [36]). 


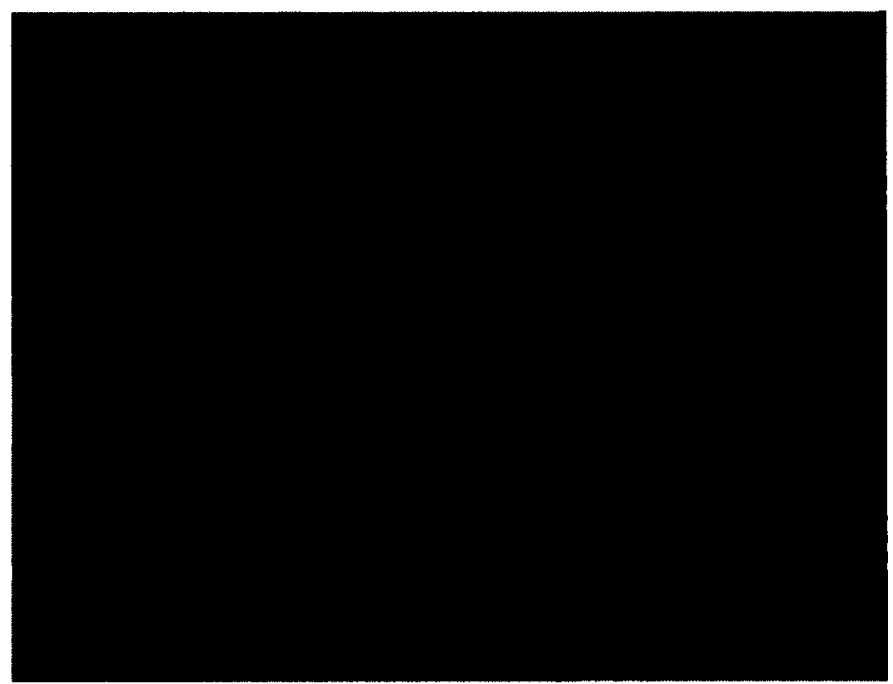

Figure 6.1: Micrograph of Silicon RF CMOS $0.13 \mu \mathrm{m}$ multi-level drain line inductors with outer diameter of $91 \mu \mathrm{m}$ and spacing of $5 \mu \mathrm{m}$ and width of $10 \mu \mathrm{m}$ and number of turns $\mathrm{n}$ of 1.75 .

\subsection{Silicon CMOS RF Multi-level Inductors Imple- mentation}

On-chip inductors play a crucial role in radio frequency integrated circuits. Several factors degrade the performance of on-chip passive components at high frequencies such as skin effect, proximity effect, electric field penetration into substrate, and substrate eddy current losses [20]. The main parameters that characterize an inductor are its self-inductance value, $\mathrm{L}$, its quality factor, $\mathrm{Q}$, and its resonant frequency $f_{\text {res }}[76]$.

A good understanding of loss mechanisms is essential in designing silicon RF CMOS onchip inductors. Loss mechanisms include metal losses, substrate losses and high frequency losses [20]. The main source of the loss of the on-chip inductor is the conductive silicon substrate. At lower frequencies, metal losses are mainly determined by the sheet resistance of the process layers used to create the device [41]. At higher frequencies, skin effect, proximity effects and current crowding have a major impact on loss mechanism [119].

The skin effect drives the ac current toward the surface of the conductor [119]. Skin 
effect increases the ac resistance of the conductor leading to lower Q. Eddy current substrate losses are caused by the magnetic field of the conductor inducing a current in the substrate [119].

Strong mutual coupling in multi-layer inductors allows the generation of the same inductance in less area as compared to planar inductors [33]. In distributed broadband amplifiers, a large number of inductors are necessary, leaving a small silicon area for each [17]. A good candidate for this purpose is the stacked structure shown in Figure 6.1, where two planar spirals are placed in series each on different metal layer.

Multi-level inductors can be implemented by connecting two or more layers in series [109]. This will let the same silicon area to be realized with an increased inductance value. Since the current flows through both metal layers in the same direction, the magnetic flux lines in the same direction result in higher mutual inductance. The flux through the two windings will strengthen one another and the total inductance of a stacked spiral consists of the sum of the two planar spiral inductances plus their mutual inductance [109]. One drawback with this approach is the lower self-resonant frequency resulting from the large feed through capacitance between the layers [112].

Square inductors are generated easily with ASITIC [120]. The physical parameters have to be determined, including number of turns $\mathrm{N}$, conductor width $\mathrm{W}$, the edge-to-edge spacing between adjacent turns, S, and the outer diameter, OD. The geometrical parameters were chosen to realize an inductance value while minimizing parasitic capacitance and area. ASITIC generates the inductor pi model parameters of spiral inductors through electromagnetic field solutions at a given frequency [120]. The layout is then imported to Cadence and ADS simulation environments.

Separation between on-chip spiral inductors should be large enough to minimize magnetic coupling effects between them as shown in Figure 6.2 and Figure 6.1 [121]. On-chip separation between inductors involves spacings that is a function of the layout size of the inductor [29]. Surrounding the CMOS $0.13 \mu \mathrm{m}$ multi-level inductors is a metallization ground shield rings as shown in Figure 6.2 which acts as the path for the return current in the structure and to reduce the substrate loss. The guard ring consists of $\mathrm{P}+$ or $\mathrm{N}+$ diffusion regions connected to ground. The guard ring prevents eddy currents from being induced in the substrate. The staggered transmission-line gate $(450 \mathrm{pH})$ and drain $(900 \mathrm{pH})$ 


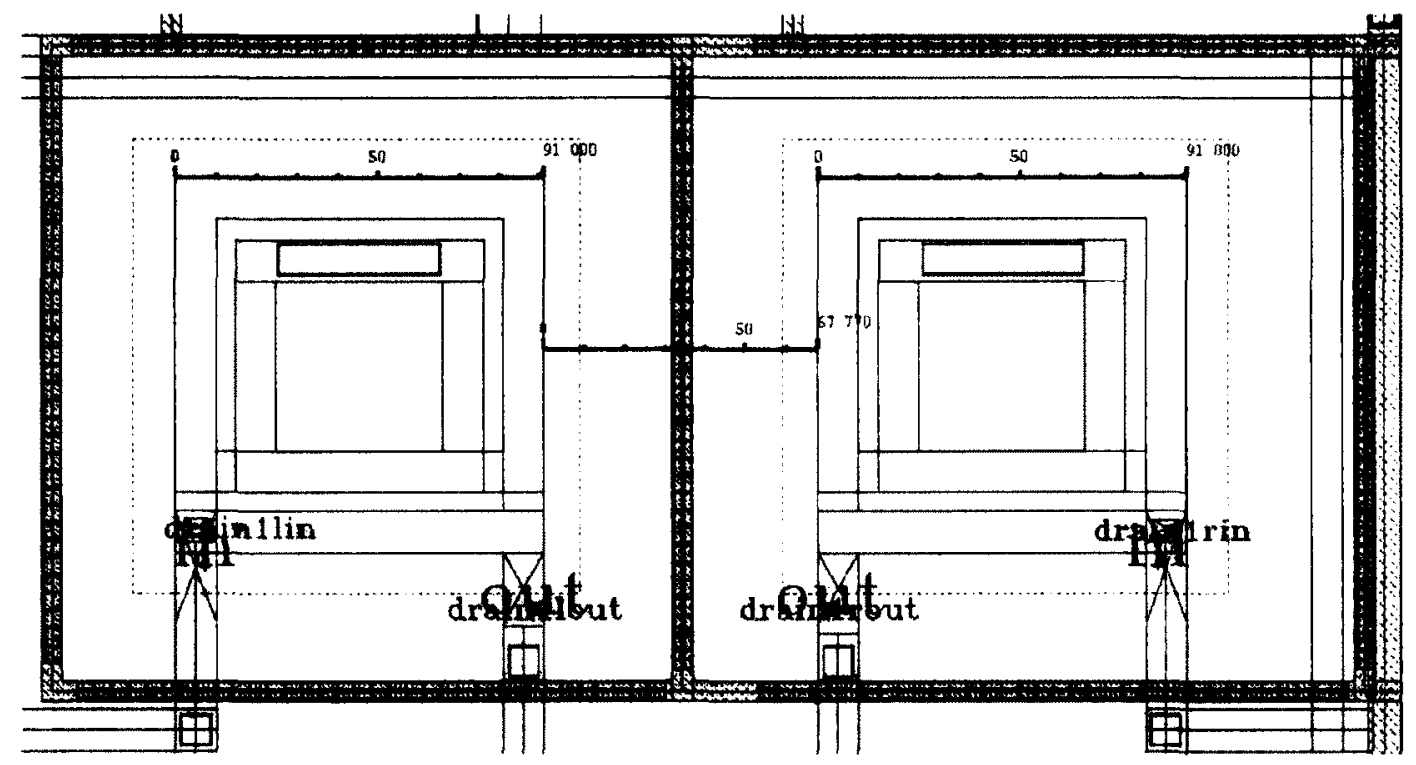

Figure 6.2: Cadence layout of silicon RF CMOS $0.13 \mu \mathrm{m}$ multi-level inductor.

on-chip inductors are modeled using both Ansoft's High Frequency Structure Simulator (HFSS) and Momentum ADS.

\subsection{CMOS Bidirectional Distributed Amplifier Cross- coupled Compensator Gain Cell Layout}

Layout techniques such as interdigitated transistor layouts, symmetry for transmissionline differential paths, multiple finger transistor layout to minimize gate resistance ( [34], [36]). Guard rings were placed surrounding the devices and inductors to reduce noise. All these RF layout techniques adopted to enhance the CMOS $0.13 \mu \mathrm{m}$ linearized bidirectional distributed amplifier performance as shown in Figure 6.3.

Minimizing the MOSFET gate resistance was achieved by properly sizing the finger width used during the layout of the transistor to $W_{\text {finger }}=4 \mu \mathrm{m}$. Another important consideration in the linearized CMOS bidirectional distributed amplifier layout is the series gate resistance of the MOS transistor since it reduces gain significantly at higher frequencies. To prevent this effect, the gate poly was split into many fingers as depicted in Figure 6.3 


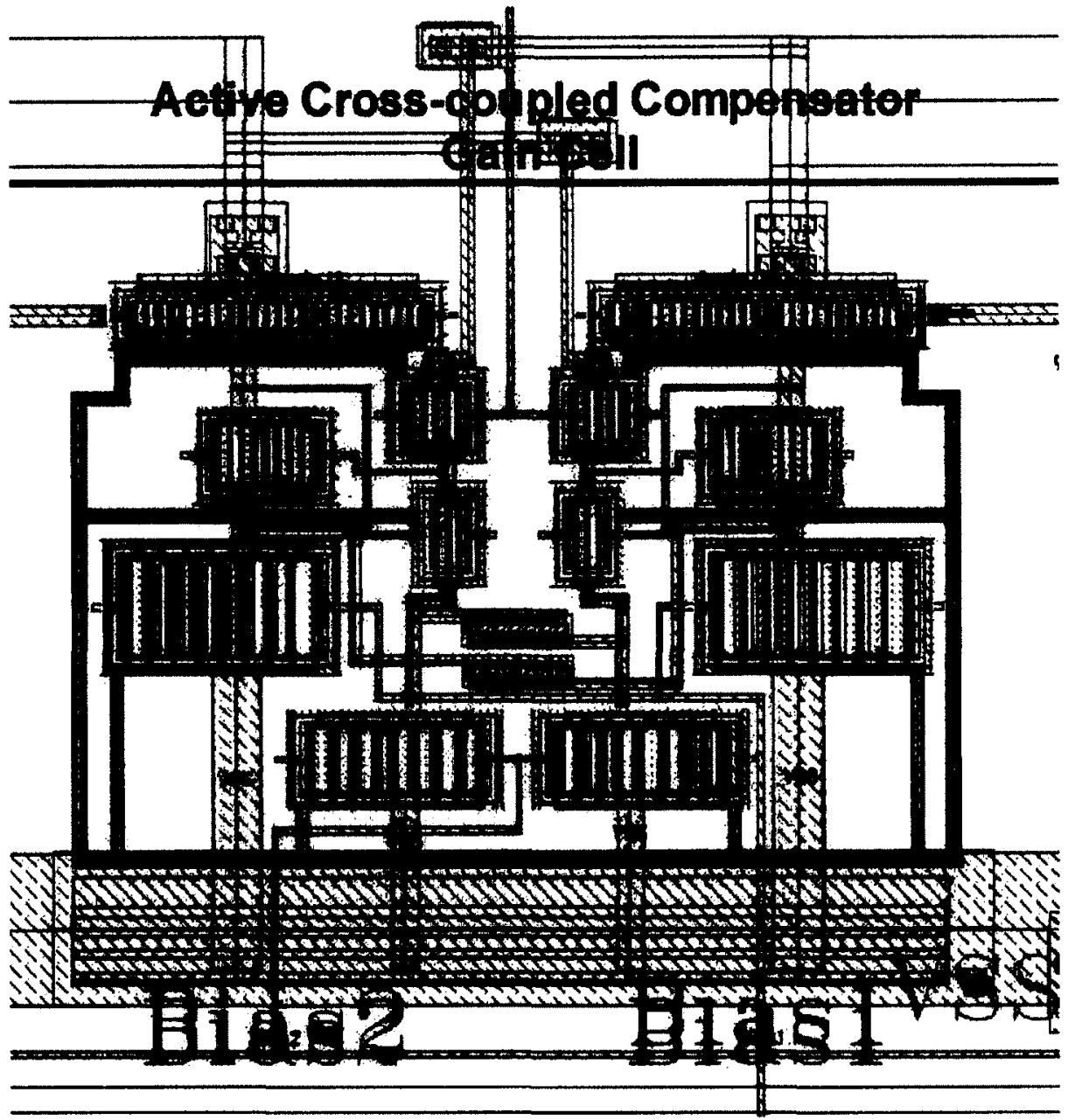

Figure 6.3: Silicon RF CMOS $0.13 \mu \mathrm{m}$ linearized bidirectional distributed amplifier full active cross-coupled compensator gain cell with enhanced tunability. 
in order to keep the total resistance down as shown in Figure 6.4.

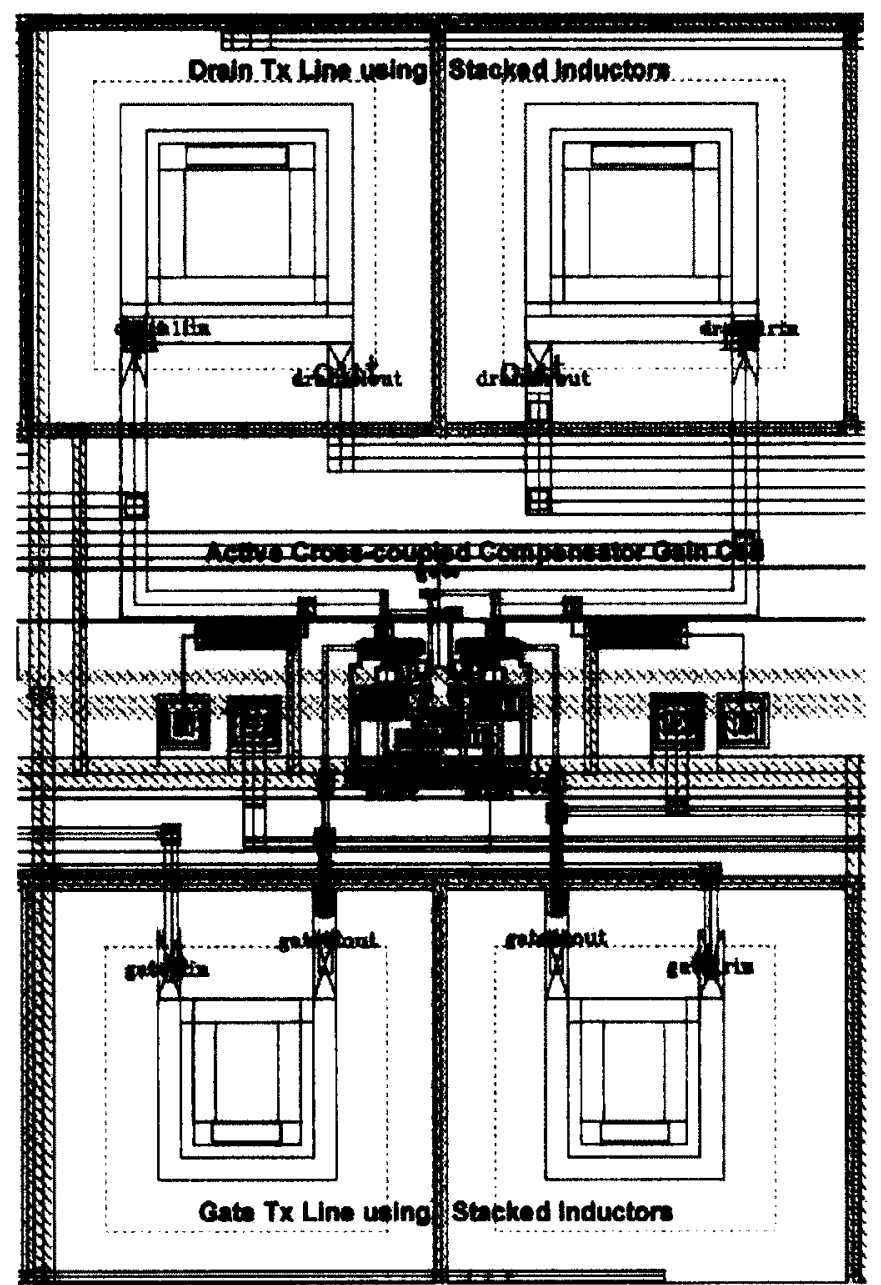

Figure 6.4: Silicon RF CMOS $0.13 \mu \mathrm{m}$ linearized bidirectional distributed amplifier crosscoupled compensator gain cell with multi-level inductors.

\subsection{Linearized CMOS Bidirectional Distributed Am- plifier Full Layout}

Most of the interconnections are done at the top metal eight layer (MA) which has the highest conductivity as shown in Figure 6.5. The first metal layer was used as a ground 


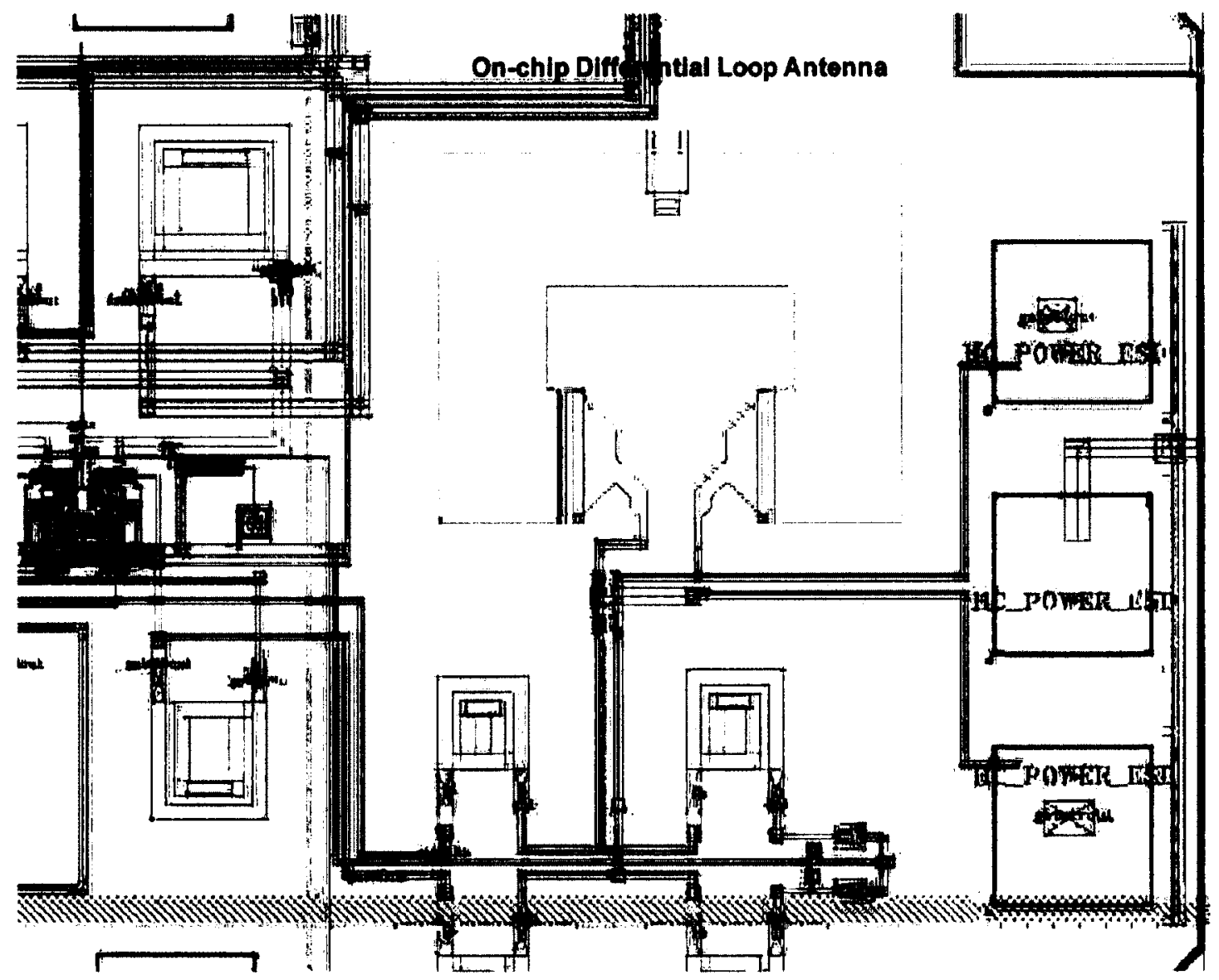

Figure 6.5: Silicon RF CMOS $0.13 \mu \mathrm{m}$ linearized bidirectional distributed amplifier codesigned with on-chip antenna. The on-chip differential loop antenna with dimension $280 \mu \mathrm{m} \times 220 \mu \mathrm{m}$ designed in HFSS. 


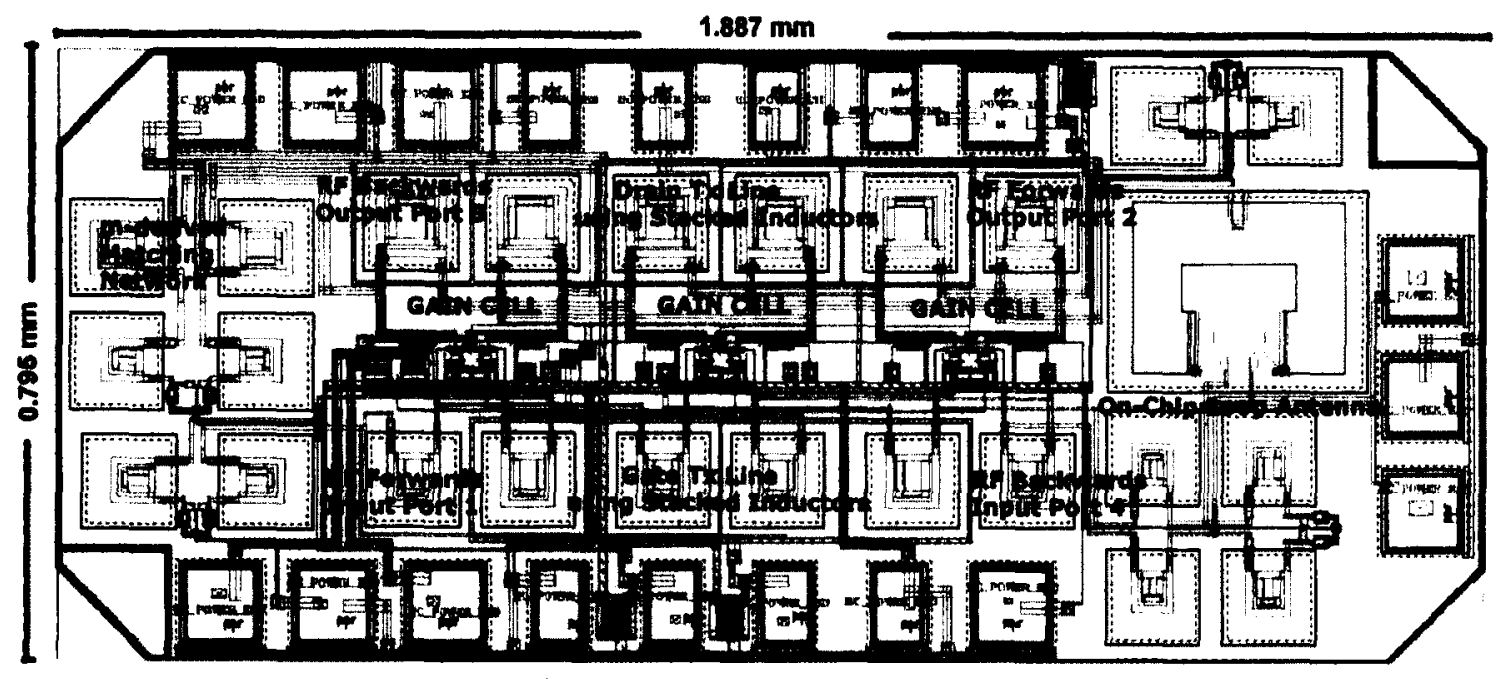

Figure 6.6: Fully-integrated silicon RF CMOS $0.13 \mu \mathrm{m}$ linearized bidirectional distributed amplifier layout co-designed with on-chip antenna with multi-level inductors. The total chip silicon area is $1.5 \mathrm{~mm}^{2}$ including testing pads.

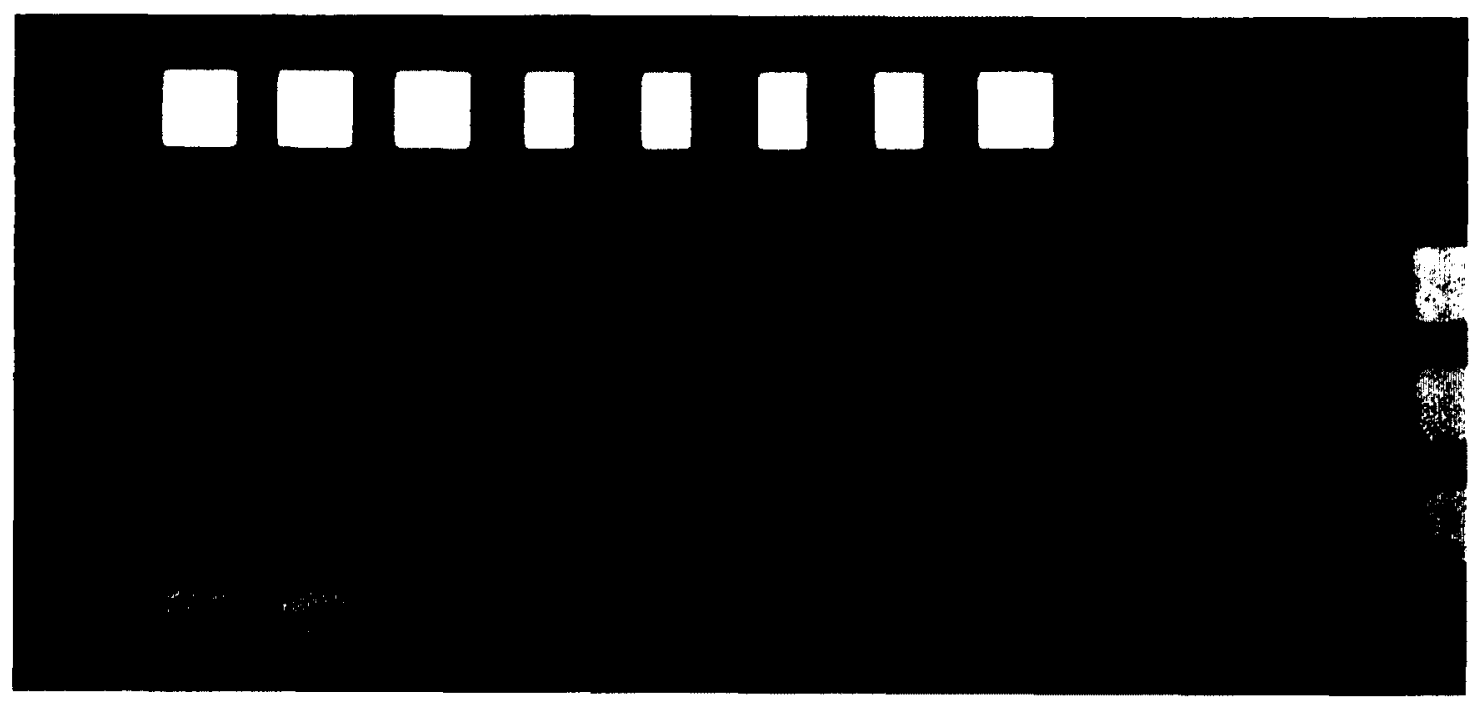

Figure 6.7: Micrograph of fully-integrated fully-differential silicon RF CMOS 0.13 $\mu \mathrm{m}$ linearized bidirectional distributed amplifier co-designed with on-chip antenna. 
plane. The layout of the gain cell blocks are done symmetrically as can be seen in Figure 6.6. The width of metal interconnections was chosen according to the amount of current flowing through them. In order to meet pattern density requirements for the layout, filling cells are placed in the blank space surrounding the gain cells as shown in Figure 6.6 and Figure 6.7. Eight pin probing pads are used in Cadence layout design of the linearized bidirectional amplifier.

As can be seen in the layout in Figures 6.6 and Figure 6.7, the chip area is dominated by the passive on-chip inductors components. The micrograph of the proposed fully-integrated fully-differential silicon RF CMOS $0.13 \mu \mathrm{m}$ linearized bidirectional distributed amplifier codesigned with the on-chip antenna is shown in Figure 6.7. The total chip silicon area is $1.887 \times 0.795 \mathrm{~mm}^{2}$ including testing pads.

\subsection{Chapter Summary}

In this chapter, high-frequency layout design techniques used in implementing the fullyintegrated linearized distributed bidirectional amplifier in silicon RF CMOS $0.13 \mu \mathrm{m}$ process have been discussed. The circuit elements forming the linearized CMOS distributed bidirectional amplifier were presented in terms of their physical arrangement and layout. 


\section{Chapter 7}

\section{Linearized CMOS Distributed Bidirectional Amplifier Experimental Setups and Chip Measurement Results}

\subsection{Introduction}

This chapter presents the experimental test setups for the fully-integrated linearized CMOS RF $0.13 \mu \mathrm{m}$ bidirectional distributed amplifier. The chip measurements that are carried out include small-signal s-parameters such as gain and return loss, harmonics power measurements such as 1-dB compression, IMD (intermodulation distortion) and noise figure measurements. All RF measurements are performed with on-wafer probing.

\subsection{High-Frequency On-Wafer Measurement System}

All RF measurements were carried out with on-wafer probing. On-wafer probing as shown in Figure 7.2 and Figure 7.3 was performed on an analytical probe station eliminating all of the problems inherent in packaging removing any uncertainty about bond wire inductances. The measurement test bench setup included network analyzers, high-frequency wafer probes and programmable DC source units.

Automated chip measurement characterization are carried out with test instruments connected using GPIB. To minimize test time, matlab scripts were compiled to control the instruments. The results are instantly graphed to view acquired measurement data from 


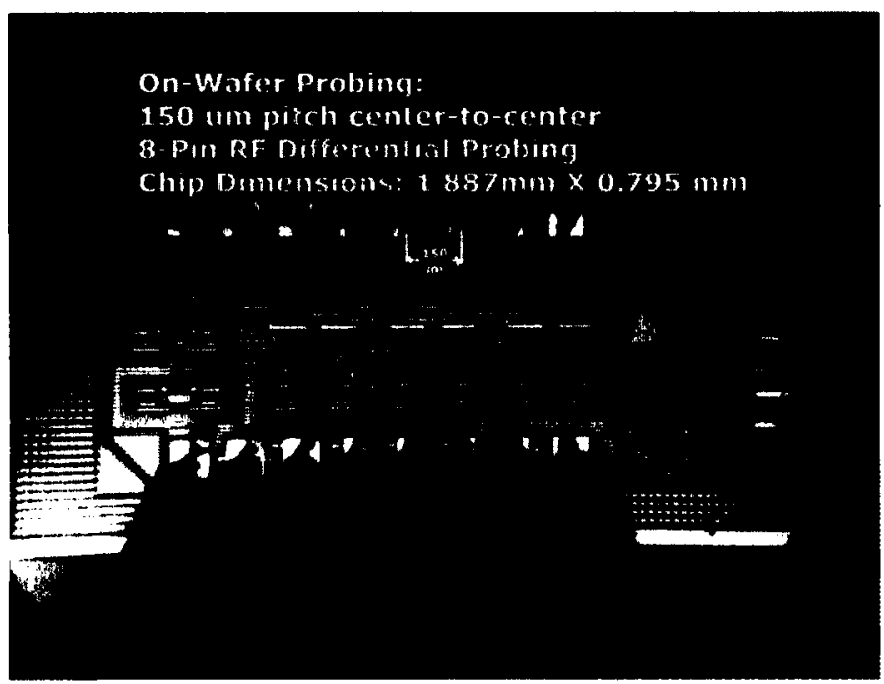

Figure 7.1: On-wafer RF automated GPIB chip testing of the $0.13 \mu \mathrm{m}$ RF CMOS linearized CMOS distributed bidirectional amplifier.

test equipment through the GPIB interface.

The testing instruments include an RF signal generator, a power meter and Agilent RF signal generator as shown in the Figure 7.2. The testing instruments also includes an Agilent RF spectrum analyzer for characterizing frequency content, $a$ vector network analyzer, modulation analyzers for characterizing modulated content in the presence of undesired blocking signals such as those from simulated cellular base stations, other cellphones, and broadcast radio stations as shown in the Figure 7.2. The measurements were conducted utilizing 8-pin high-frequency wafer probes.

\subsection{S-parameter and Harmonics Power Measurements}

The S-parameter test setup is shown in Figure 7.3 and includes DC-supplies, 8-pin high frequency probes and a vector network analyzer. The S-parameter measurements were carried out using the Anritsu 37347C Vector Network Analyzer and multi-contact wedge 8-pin high frequency differential probes with $2 \mathrm{RF}$ needles up to $40 \mathrm{GHz}$. The network analyzer is capable of accurately extracting the S-parameters of the device up to $20 \mathrm{GHz}$. The Anritsu $37347 \mathrm{C}$ was calibrated up to the probes with the Open-Short-Load-Through calibration method ([119], [122]). A full 2-port calibration from a start frequency of 50 


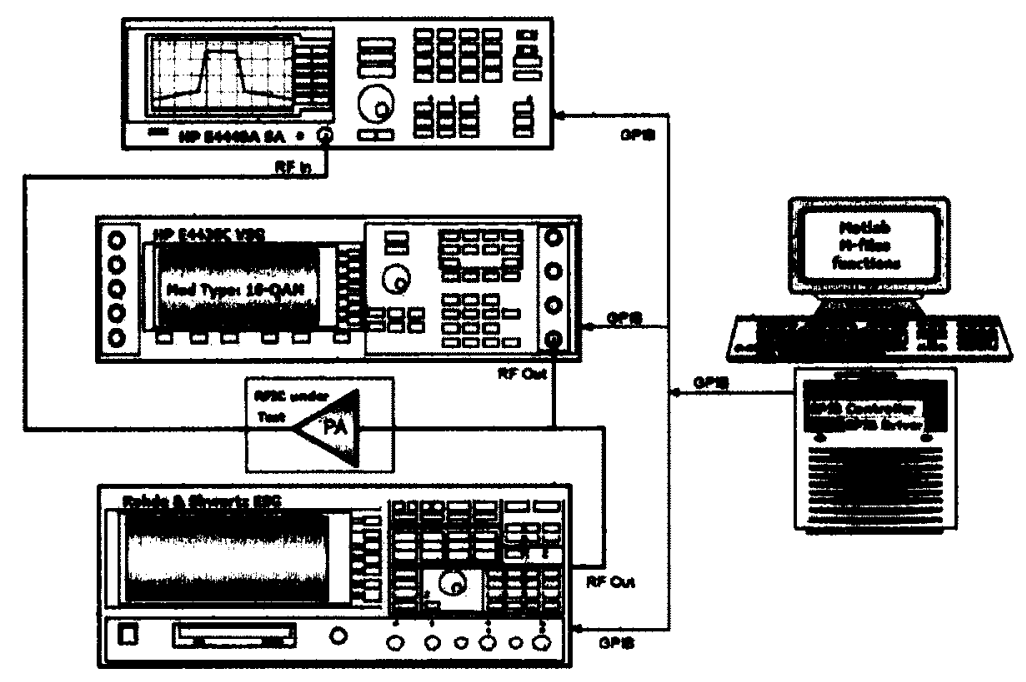

Figure 7.2: RF automated GPIB testing of the $0.13 \mu \mathrm{m}$ RF CMOS three-stage fullydifferential linearized CMOS distributed bidirectional amplifier with high-frequency RF probes.

$\mathrm{MHz}$ to a stop frequency of $20 \mathrm{GHz}$ are performed. The device under test was connected as shown in Figure 7.3. The measured data collected using network analyzer in the form of S-parameter relating the electromagnetic waves scattered from the device under test to those EM waves incident upon the vector network analyzer.

To characterize the linearity of the linearized CMOS bidirectional distributed amplifier, one-tone and two-tone power measurements were conducted for characterizing 1-dB compression point $P_{1 d B}$ and third-order interception point (IP3) respectively ( [68], [22]). The output power measurement setup is shown in Figure 7.4. IIP3 measurements used two Rohde and Shwartz tone signal generators and a high-frequency power combiner with the $8975 \mathrm{~A}$ spectrum analyzer.

An external $12 \mathrm{GHz}$ power combiner was used to combine the tones from signal generators. At the output stage, the RF signal was fed to a spectrum analyzer which provided a load resistance of $50 \Omega$. On-wafer two-tone IMD3 measurements was performed. The 8975A spectrum analyzer was used for single-tone compression measurements as well.

Two-tone power measurements were conducted to characterize the third-order input 


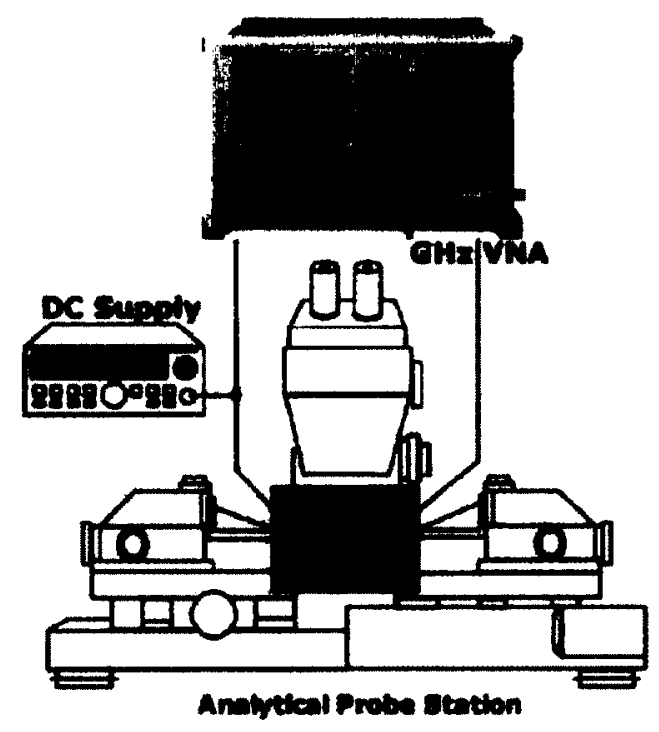

Figure 7.3: Test setup for S-parameter measurements for the linearized CMOS distributed bidirectional amplifier.

interception point (IIP3) of the linearized CMOS bidirectional DA. IIP3 comparison of measured output power versus input power with and without linearization is shown in Figure 7.5. A measured IIP3 improvement of $10 \mathrm{~dB}$ at $5 \mathrm{GHz}$ with $100 \mathrm{MHz}$ spacing is achieved for both forward and reverse RF paths. The CMOS linearized bidirectional amplifier achieves a highly linear output power of $18.5 \mathrm{dBm}$.

The small-signal S-parameters measurements were carried out using an Anritsu 37347C vector network analyzer with proper calibration techniques. The measured RF forward path and reverse path S-parameters for the linearized CMOS bidirectional DA achieves a measured peak gain of $5 \mathrm{~dB}$ and unity gain bandwidth of $9.5 \mathrm{GHz}$ as shown in Figure 7.6 (a) and (b) for both forward and reverse RF paths. The measured input and output return losses are better then $-10 \mathrm{~dB}$ over the frequency range with better than $-26 \mathrm{~dB}$ isolation.

The IM3 distortion reduction measurement of the linearized bidirectional amplifier was examined at four different two-tones frequencies at $(1 \mathrm{GHz}, 3 \mathrm{GHz}, 5 \mathrm{GHz}$ and $5.9 \mathrm{GHz}$ ) with $100 \mathrm{MHz}$ spacing using an Agilent E4440A spectrum analyzer. A comparison of the measured output spectra with and without linearization for these frequencies is shown 

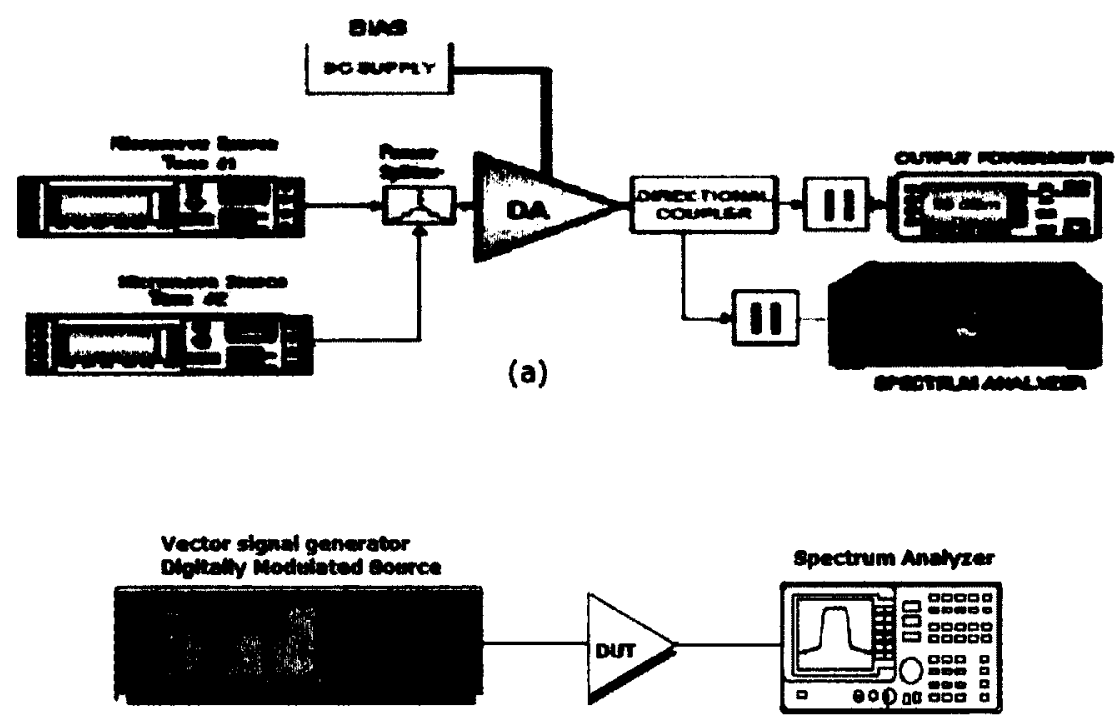

(b)

Figure 7.4: (a) Two-tone test setup for measuring power and intermodulation distortion (b) Vector digitally modulated signals generator measurement setup. 

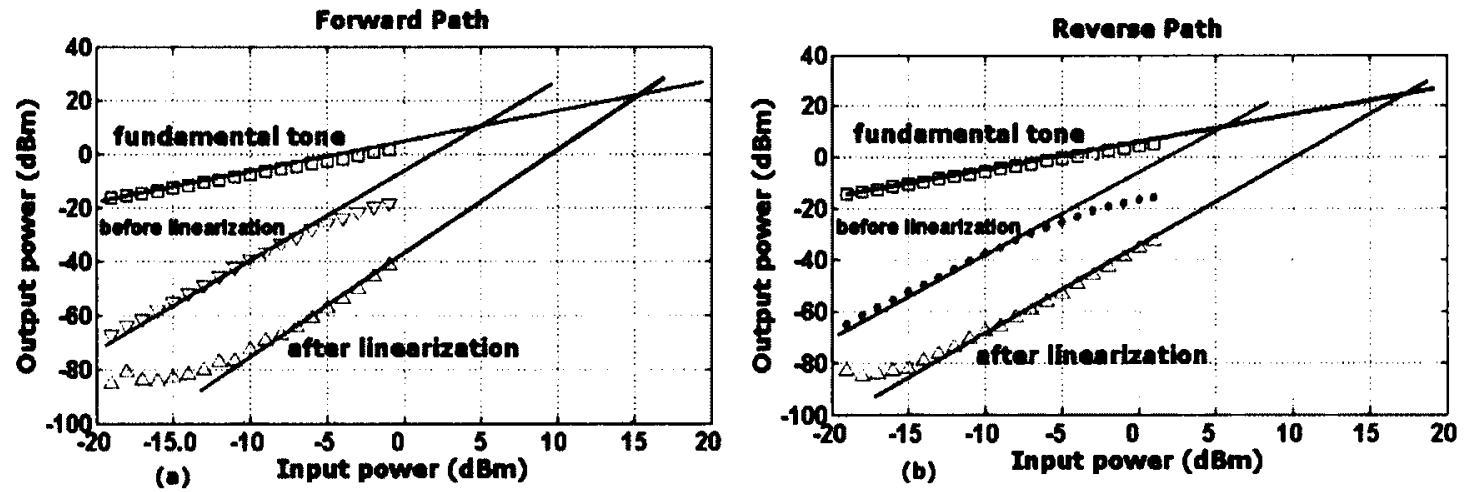

Figure 7.5: IIP3 Comparison of measured reverse path output power versus input power with and without linearization for both (a) forward and (b) reverse RF paths.

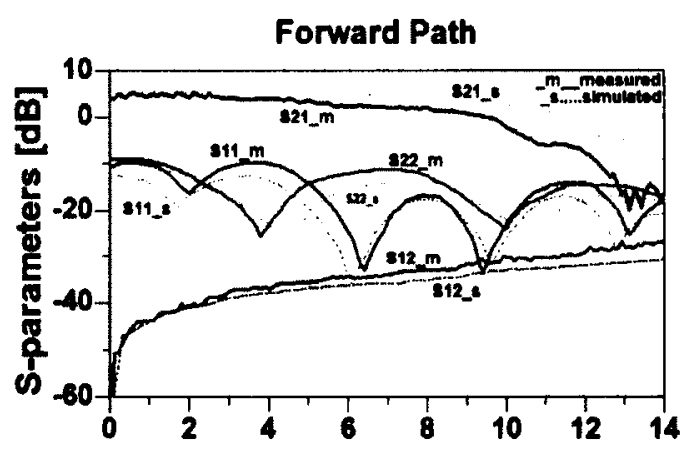

(a) Frequency [GHz]

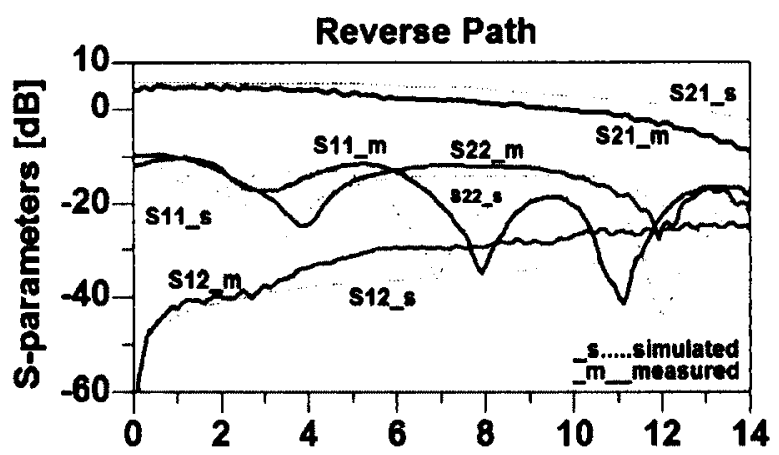

(b) Frequency $[\mathrm{GHz}]$

Figure 7.6: Comparison of simulation and measured differential S-parameters for both (a) forward and (b) reverse RF paths.

in Figure 7.7, Figure 7.8, Figure 7.9 and Figure 7.10. Intermodulation distortion (IMD) nonlinearity appears as a result of applying two tones to the distributed bidirectional amplifier. The measured IM3 distortion reduction is $20 \mathrm{~dB}$ for both forward and reverse RF paths. The intermodulation distortion (IMD) nonlinearity improvement match the simulation results. A comparison of the measured IM3 distortion reduction with and without linearization over broadband frequency of operation for both RF paths is shown in Figure 7.11. The linearization over broadband frequency measurement characterization were carried out with both RF signal generators and the Agilent E4440A spectrum analyzer test instrument connected using GPIB. The intermodulation distortion (IMD) nonlinearity 

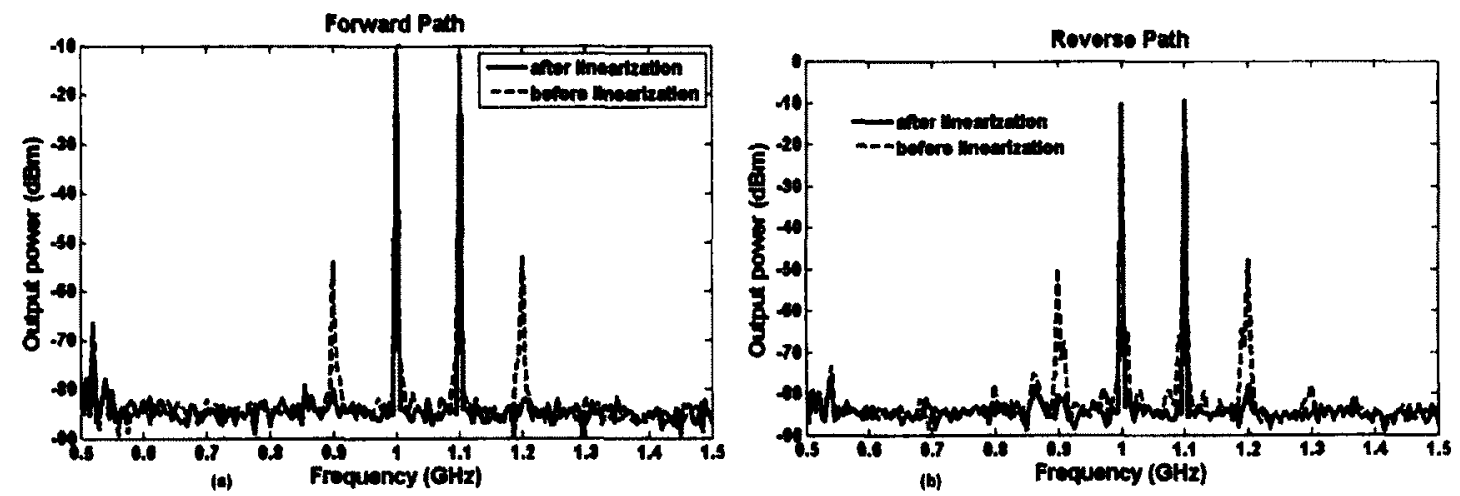

Figure 7.7: Comparison of measured output spectra with and without linearization for two-tones frequencies at $1 \mathrm{GHz}$ with $100 \mathrm{MHz}$ spacing for both (a) forward and (b) reverse RF paths.

results are instantly graphed to view acquired measurement data from spectrum analyzer test equipment.

Unmodulated carrier signals have been used as stimuli for distortion measurements. However, digitally-modulated stimulus signals are used as well as they provide more realistic measurement results [123]. For a digitally modulated carrier, distortion produces spectral regrowth. Nonlinear spectral analysis with digitally modulated signal as input was carried out [123]. To demonstrate the linearization capability of the bidirectional amplifier, it was measured using an Agilent E4438C ESG vector signal generating communicationspecific test signals for testing wireless communication systems such as $\Pi / 4$-differential QPSK (DQPSK) modulated signals [123]. These modulation schemes are commonly used in many commercial wireless systems on the market today due to their spectral efficiency and simple modulator/demodulator construction. A performance test was done using a narrowband $\Pi / 4-D Q P S K$ signal. The signal used for this test is a standard $\Pi / 4-D Q P S K$ signal at $125 \mathrm{ksymbols} / \mathrm{s}$ ( $250 \mathrm{kbits} / \mathrm{s}$ ) and was pulse shaped using a square root Nyquist $40 \mathrm{MHz}$ filter using a roll-off factor $\beta=0.35$.

Measured normalized output power spectral density PSD with and without linearization at $2 \mathrm{GHz}$ for a standard $\Pi / 4$-DQPSK digitally modulated carrier signal is shown in Figure 7.12. We can see that the nonlinearity of the amplifier has caused the output signal to be spread in frequency and the third order nonlinearity has caused the first set of 

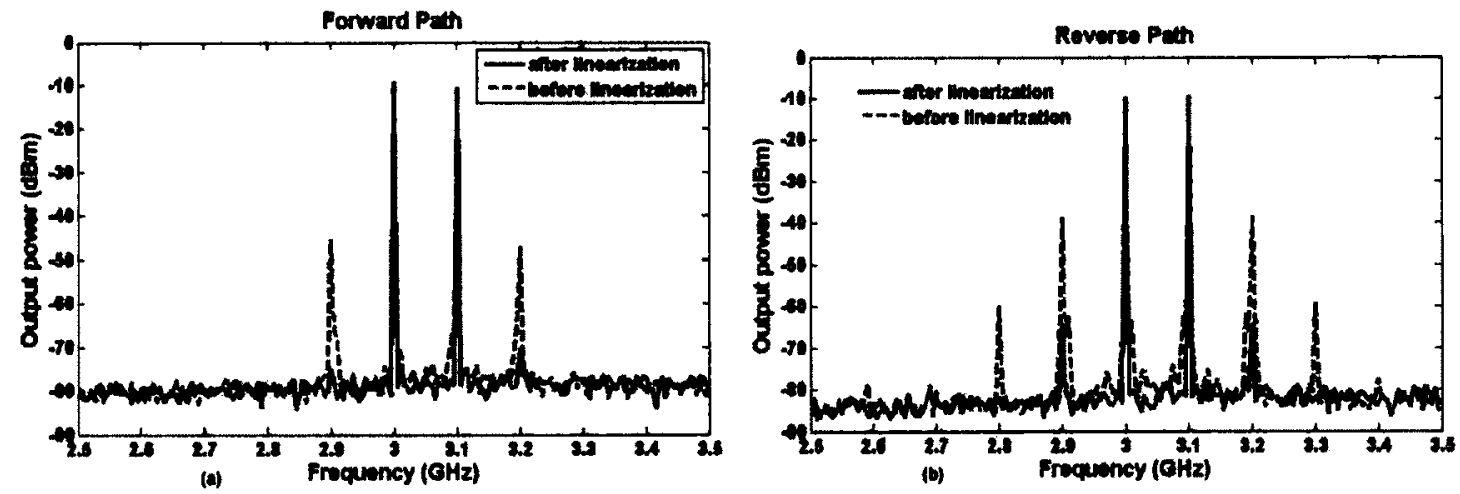

Figure 7.8: Comparison of measured output spectra with and without linearization for two-tones frequencies at $3 \mathrm{GHz}$ with $100 \mathrm{MHz}$ spacing for both (a) forward and (b) reverse RF paths.

shoulders on the output signal, which are around $45 \mathrm{~dB}$ down from the signal peak. This output signal is unacceptable from a system perspective, as the distortion spills out of the channel used by the signal and into nearby channels that are occupied by signals from other users. This is known as adjacent channel interference, and reduces the spectral efficiency of the system. Spectral regrowth due to amplifier nonlinearities are fully compensated for, resulting in a $20 \mathrm{~dB}$ improvement in adjacent channel interference relative to the output signal.

To further demonstrate the performance of the linearized bidirectional DA, using an Agilent E4438C ESG vector signal generator a custom multi-tone waveform test was generated using a 10-Tones $5 \mathrm{MHz}$ bandwidth each [123]. Measured normalized output PSD with and without linearization at $2 \mathrm{GHz}$ for Multi-tone modulated signal is shown in Figure 7.13. A $20 \mathrm{~dB}$ of improvement is achieved through linearization.

QAM is another common modulation technique used in modern communications systems due to its bandwidth efficiency. The measured normalized output PSD with and without linearization at $2 \mathrm{GHz}$ for 4-QAM digitally modulated carrier signal is shown in Figure 7.14.

The varactor-based active post distortion enables one to tune the IM3 reduction with ease and precision. It provides distortion cancellation tuning at various combination of 

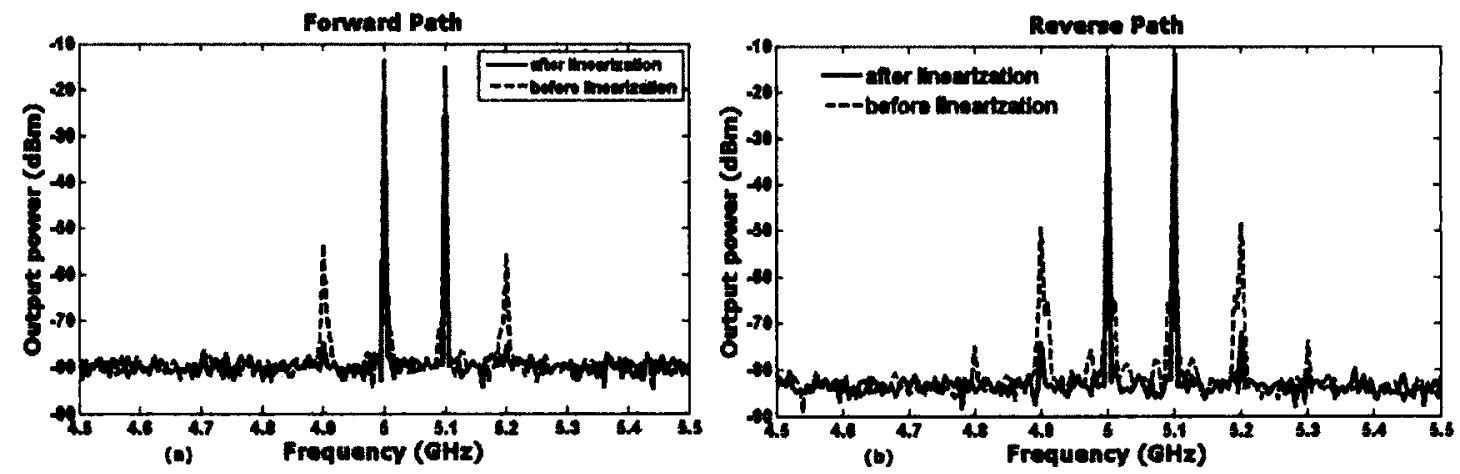

Figure 7.9: Comparison of measured reverse path output spectra with and without linearization for two-tones frequencies at $5 \mathrm{GHz}$ with $100 \mathrm{MHz}$ spacing for both (a) forward and (b) reverse $R F$ paths.

varactor voltages. The varactor bias voltage is adjusted from $1 \mathrm{~V}$ to $1.8 \mathrm{~V}$. An optimum bias condition is observed for IM3 distortion suppression which is formed at $1.45 \mathrm{~V}$ as can be seen in Figure 7.15.

\subsection{Noise Figure Setup and Measurement}

The noise figure test setup includes noise figure analyzer N8975A, high-frequency noise source, DC-supplies, device under test, RF 8-pin wafer probes as shown in Figure 7.16. A noise figure measurement of the linearized CMOS bidirectional distributed amplifier was performed. After calibration, the measurements indicate that the noise figure of the amplifier shows a minimum of $7.8 \mathrm{~dB}$ at around $1 \mathrm{GHz}$ and is below $9 \mathrm{~dB}$ for frequencies up to $9.5 \mathrm{GHz}$ as shown in Figure 7.17 .

A summary comparing previous state-of-the-art published measured linearized DAs is presented in Table 7.1. Table 7.1 highlights the proposed linearized bidirectional DA large $20 \mathrm{~dB}$ IMD3 reduction over broadband frequency range for both RF paths with the least power consumption and minimum silicon chip area compared to other published resutls. Table 7.1 presents all the previously published Linearized DA's to the author's knowledge. It shows that the proposed linearized DA has the lowest power consumption and minimum silicon chip area in comparison to all the previously published linearized DAs. The proposed linearized DA is implemented with 3-stage gain cells to optimize for 

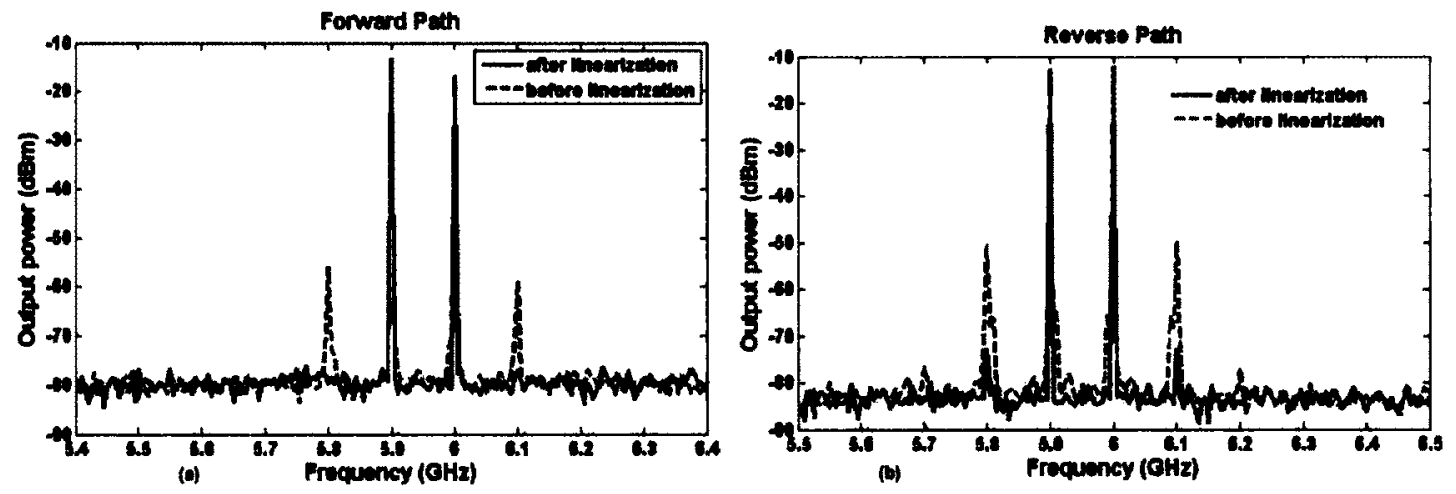

Figure 7.10: Comparison of measured output spectra with and without linearization for two-tones frequencies at $5.9 \mathrm{GHz}$ with $100 \mathrm{MHz}$ spacing for both (a) forward and (b) reverse RF paths.
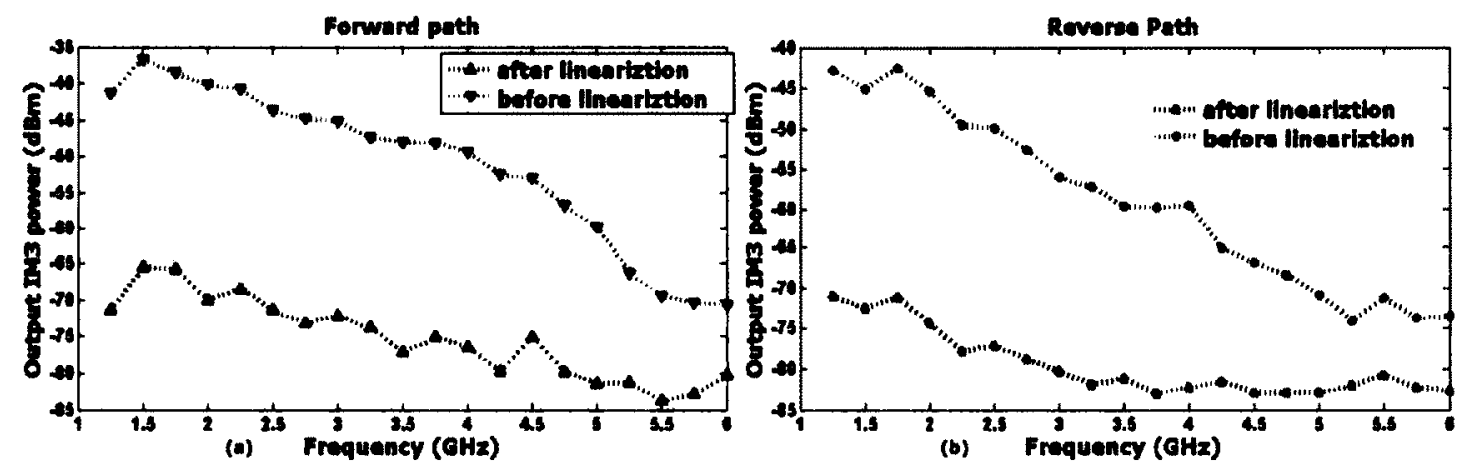

Figure 7.11: Comparison of the measured IM3 distortion reduction with and without linearization over broadband frequency of operation for both (a) forward and (b) reverse RF paths.

silicon chip area of $1.5 \mathrm{~mm}^{2}$. The proposed linearized bidirectional DA power consumption is $128 \mathrm{~mW}$. Comparison with state-of-the-art previously published measured linearized distributed amplifiers the proposed linearized bidirectional DA consumes the least power consumption as shown in Table 7.1.

Several linearized DAs have been published ( [7]- [124]) and ( [66], [67]) as shown in Table 7.1, however most of the published DA linearization methods reported do not provide large IM3 distortion reduction. Since they involve system-level linearization with bulky discrete components which is not suited for fully-integrated circuit miniaturization. Due to the discrete component performance variation with frequency, they also suffered 
Table 7.1: Comparison with state-of-the-art previously published measured linearized distributed amplifiers highlighting the large $20 \mathrm{~dB}$ IMD3 reduction over broadband frequency range for both RF paths with least power consumption and minimum silicon chip area for the proposed bidirectional fully-differential fully-integrated solution.

\begin{tabular}{|c|c|c|c|c|c|c|c|c|c|}
\hline $\begin{array}{l}\text { Design } \\
\text { Ref. }\end{array}$ & $\begin{array}{l}\text { Technology } \\
\text { Process }\end{array}$ & $\begin{array}{l}\text { Power } \\
\text { Gain } \\
(\mathrm{dB})\end{array}$ & $\begin{array}{l}\text { Operational } \\
\text { Bandwidth } \\
(\mathrm{GHz})\end{array}$ & $\begin{array}{l}\text { IMD3 } \\
\text { Reduction } \\
\text { (dB) }\end{array}$ & $\begin{array}{l}\text { IIP3 } \\
(\mathrm{dBm}) \\
\end{array}$ & $\begin{array}{l}\text { Power } \\
\text { Consump. } \\
(\mathrm{mW})\end{array}$ & $\begin{array}{l}\text { Linearization } \\
\text { Technique }\end{array}$ & $\begin{array}{l}\text { Circuit } \\
\text { Topology } \\
\text { Chip Area } \mathrm{mm}^{2}\end{array}$ & $\begin{array}{l}\text { Differential or } \\
\text { Single Ended }\end{array}$ \\
\hline$[17]$ & $\begin{array}{l}\text { GaAs } \\
\text { HEMT }\end{array}$ & $\mathrm{NA}$ & 10 & 15 & $\mathrm{NA}$ & $\overline{\mathrm{NA}}$ & Feedforward & $\begin{array}{c}\text { Discrete } \\
\text { Components }\end{array}$ & $\begin{array}{l}\text { Single } \\
\text { Ended }\end{array}$ \\
\hline [7] & $\begin{array}{l}\text { SiGe } \\
\text { BJT }\end{array}$ & 14 & 2.2 & 12 & $\mathrm{NA}$ & NA & $\begin{array}{c}\text { Parallel } \\
\text { Diode }\end{array}$ & $\begin{array}{c}\text { Discrete } \\
\text { Components }\end{array}$ & $\begin{array}{l}\text { Single } \\
\text { Ended }\end{array}$ \\
\hline [23] & $\begin{array}{l}\text { SiGe } \\
\text { BJT }\end{array}$ & 13 & 2.3 & 10 & 10 & 180 & $\begin{array}{c}\text { Self } \\
\text { Biased }\end{array}$ & $\begin{array}{c}\text { Discrete } \\
\text { Components }\end{array}$ & $\begin{array}{l}\text { Single } \\
\text { Ended }\end{array}$ \\
\hline [24] & $\begin{array}{c}\text { GaAs } \\
\text { MESFET }\end{array}$ & $\mathrm{NA}$ & 2.5 & 20 & $\mathrm{NA}$ & $\mathrm{NA}$ & $\begin{array}{c}\text { Derivative } \\
\text { Superposition }\end{array}$ & $\begin{array}{c}\text { Fully Integrated } \\
\text { NA } \\
\end{array}$ & $\begin{array}{l}\text { Single } \\
\text { Ended }\end{array}$ \\
\hline [25] & $\begin{array}{c}\text { InGaP } \\
\text { GaAs HBT }\end{array}$ & 7.5 & 11 & 7 & 22 & NA & $\begin{array}{c}\text { Optimum Bias } \\
\text { Condition }\end{array}$ & $\begin{array}{c}\text { Fully Integrated } \\
\text { NA } \\
\end{array}$ & $\begin{array}{l}\text { Single } \\
\text { Ended }\end{array}$ \\
\hline$[28]$ & $\begin{array}{l}\text { CMOS } \\
0.18 \mu \mathrm{m} \\
\end{array}$ & 7 & 7.5 & 5 & 19 & 158 & Multi-Tanh & $\begin{array}{c}\text { Fully Integrated } \\
2.4 \mathrm{~mm}^{2} \\
\end{array}$ & $\begin{array}{c}\text { Fully } \\
\text { Differential }\end{array}$ \\
\hline [31] & $\begin{array}{l}\text { CMOS } \\
0.18 \mu \mathrm{m}\end{array}$ & 8.4 & $3.7-8.8$ & 11 & 19 & 154 & MGTR & $\begin{array}{l}\text { Fully Integrated } \\
2.5 \mathrm{~mm}^{2}\end{array}$ & $\begin{array}{l}\text { Single } \\
\text { Ended }\end{array}$ \\
\hline $\begin{array}{l}\text { This } \\
\text { Work } \\
{[30]}\end{array}$ & $\begin{array}{l}\text { CMOS } \\
0.13 \mu \mathrm{m}\end{array}$ & 5 & 9.5 & 20 & 18.5 & 128 & $\begin{array}{l}\text { Cross-Coupled } \\
\text { Compensator }\end{array}$ & $\begin{array}{l}\text { Fully Integrated } \\
1.5 \mathrm{~mm}^{2}\end{array}$ & $\begin{array}{c}\text { Fully } \\
\text { Differential } \\
\text { Bidirectional }\end{array}$ \\
\hline
\end{tabular}




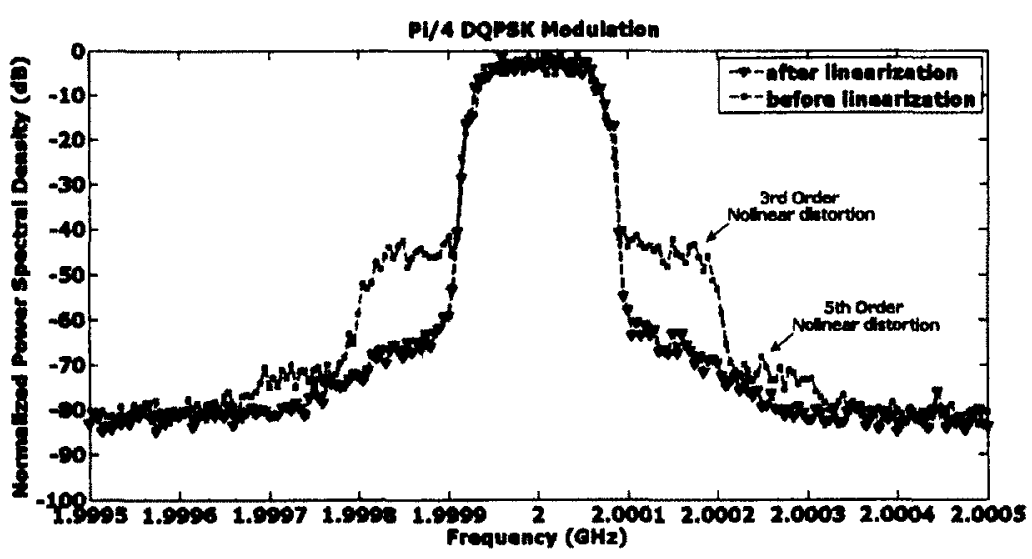

Figure 7.12: Measured output PSD with and without linearization at $2 \mathrm{GHz}$ for $\Pi / 4$ DQPSK digitally modulated carrier signal using a square root Nyquist filter using a roll-off factor $\beta=0.35$. Spectral regrowth due to amplifier non-linearities are fully compensated for, resulting in nearly $20 \mathrm{~dB}$ improvement in ACI relative to the output signal.

from limited linearization over broad bandwidth. Other DA linearization techniques have narrow linearized bandwidth at lower frequencies ( $[13],[15])$ and apply only DC-based linearization techniques.

A CMOS DA based multi-tanh linearization technique is also reported in [26]. However the CMOS DA based multi-tanh linearization technique offered a limited $5 \mathrm{~dB}$ IM3 distortion reduction [26]. Another linearized DA that has been published is a differential DA with circuit-level feedforward linearization technique [27]. It operated over a wide band from 0.1-12 GHz, however only simulation results were presented [27]. Lau and Chan proposed a linearized DA that achieved a $10 \mathrm{~dB}$ IM3 reduction over a limited $2.3 \mathrm{GHz}$ bandwidth range [23]. Recently, Lu and Pham ( [28], [29]) proposed a multi-gated transistor (MGTR) topology based CMOS linearized DA. The MGTR-based linearized distributed amplifier operated over a limited bandwidth of $4 \mathrm{GHz}$ range and had only a $11 \mathrm{~dB}$ IM3 reduction. Comparing the proposed CMOS linearized DA in this work [30] to other published ones, the proposed linearized CMOS DA offers a $20 \mathrm{~dB}$ IM3 distortion reduction with $9.5 \mathrm{GHz}$ operational bandwidth and with the least power consumption [30].

Measurement setup of of the $0.13 \mu \mathrm{m}$ RF CMOS three-stage fully-differential linearized 


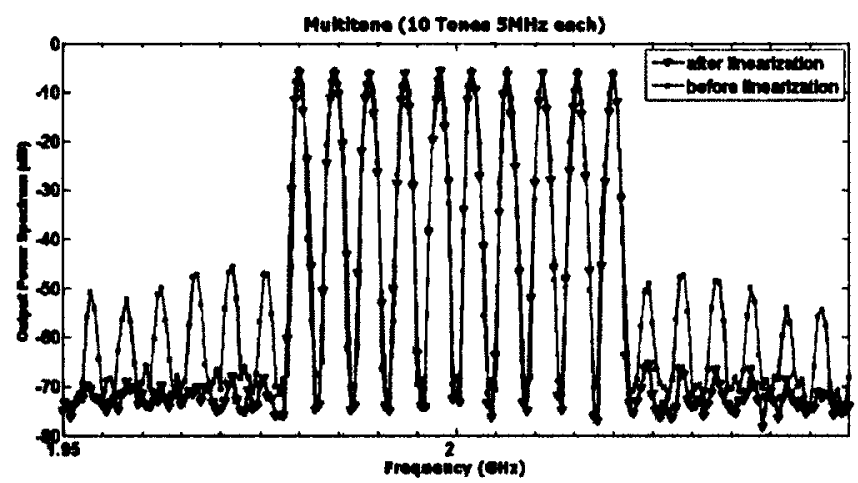

Figure 7.13: Measured output PSD with and without linearization at $2 \mathrm{GHz}$ for 10-Tones $5 \mathrm{MHz}$ bandwidth each Multi-tone modulated signal.

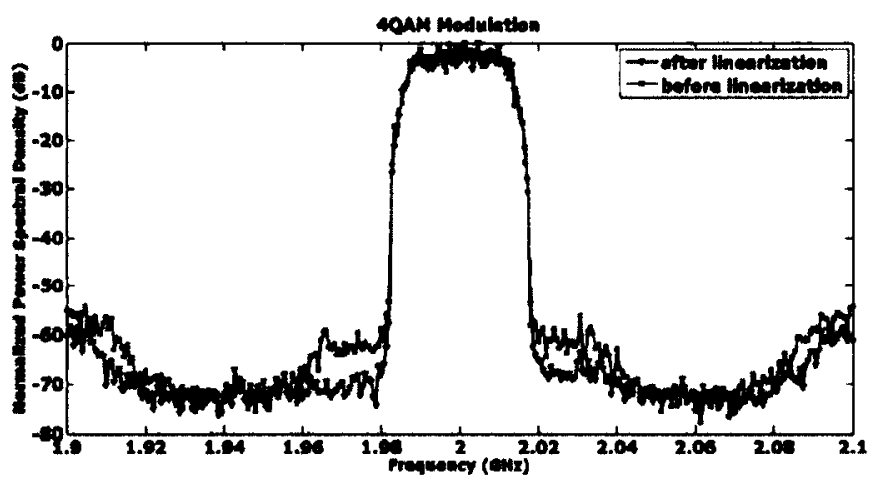

Figure 7.14: Measured output PSD with and without linearization at $2 \mathrm{GHz}$ for 4 QAM digitally modulated carrier signal.

CMOS bidirectional distributed amplifier on analytical probe station is shown in Figure 7.18. The testing instruments include an RF signal generator, a power meter and Agilent RF signal generator and programmable DC source units as shown in the Figure 7.18. The testing instruments also includes an Agilent RF spectrum analyzer for characterizing frequency content, a vector network analyzer, modulation analyzers and 8-pin high-frequency wafer probes. 


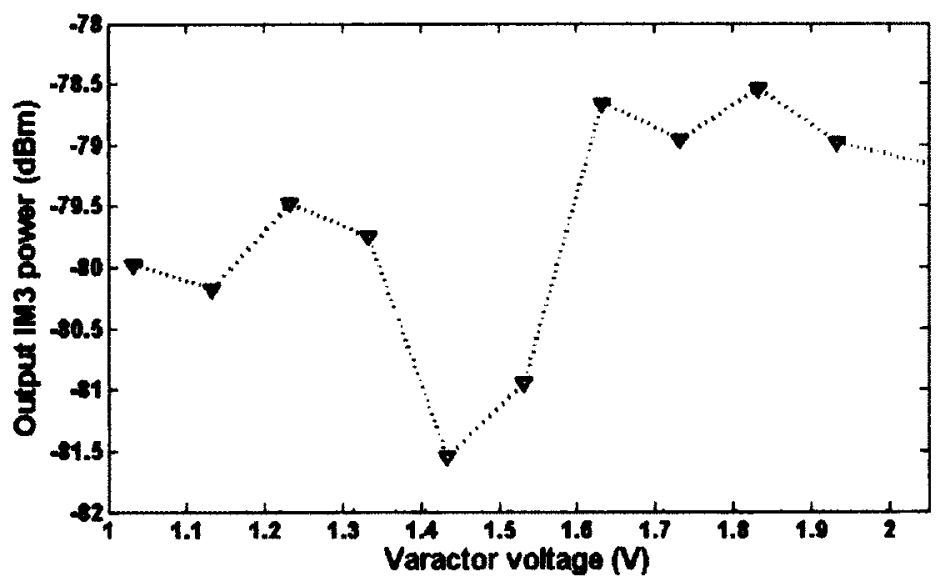

Figure 7.15: Comparison of the IM3 distortion reduction under different varactor bias voltage adjustment from $1 \mathrm{~V}$ to $1.8 \mathrm{~V}$ there exist an optimal bias at which IM3 suppression is formed at $1.45 \mathrm{~V}$.

\subsection{Summary}

In this chapter, the experimental measurement test-setups and measurement results for the fully-integrated fully-differential linearized CMOS distributed bidirectional amplifier are presented. The linearized distributed bidirectional amplifier achieves large $20 \mathrm{~dB}$ IMD3 distortion reduction over ultra-wideband frequency range for both RF paths with least power consumption and minimum silicon chip area solution compared to other published linearized DAs. The proposed fully-integrated DA linearization technique greatly suppresses the third-order intermodulation (IM3) distortion with drain and gate transmissionlines staggered to filter out the IM3 distortion. The proposed fully-differential linearized DA employs a CMOS cross-coupled compensator to enhance the linearity of the DA gain cell with a nonlinear drain capacitance compensator for wider linearization bandwidth. The proposed linearized CMOS bidirectional DA achieves a measured IM3 reduction of $20 \mathrm{~dB}$ in both $\mathrm{RF}$ directions with a two-way gain of $5 \mathrm{~dB}$ over ultra-wideband $0.1 \mathrm{GHz}$ to $9.5 \mathrm{GHz}$ frequency of operation eliminating the need of RF switches which degrade performance and increase insertion loss. The proposed linearized DA is fully-differential suppressing substrate noise thus providing better dynamic range compared to single-ended linearized DA designs and with the least power consumption of $128 \mathrm{~mW}$ and chip silicon area compared to previous published work. An IIP3 of $18.5 \mathrm{dBm}$ is achieved for both RF 


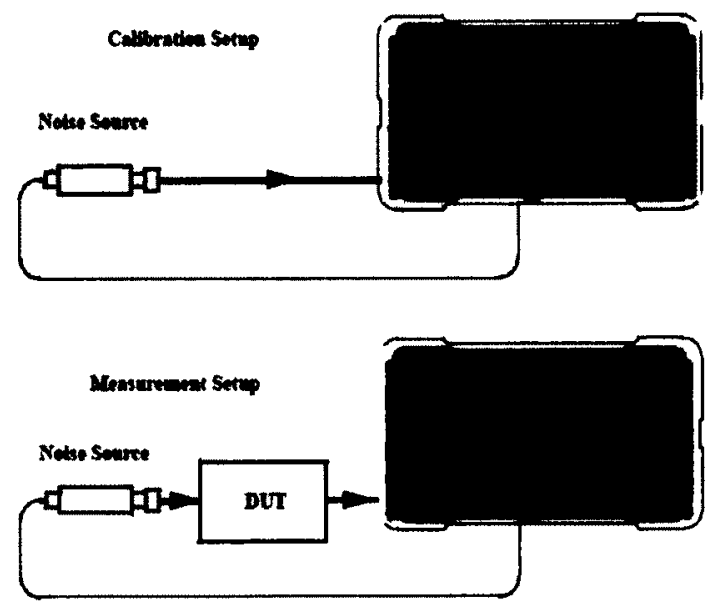

Figure 7.16: Noise figure calibration and measurement setup for the linearized CMOS distributed bidirectional amplifier.

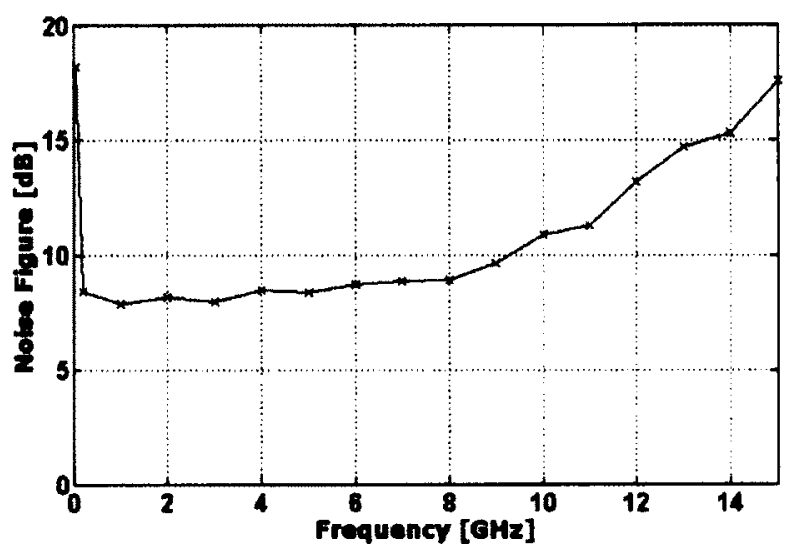

Figure 7.17: Measured noise figure for the linearized CMOS distributed bidirectional amplifier. 


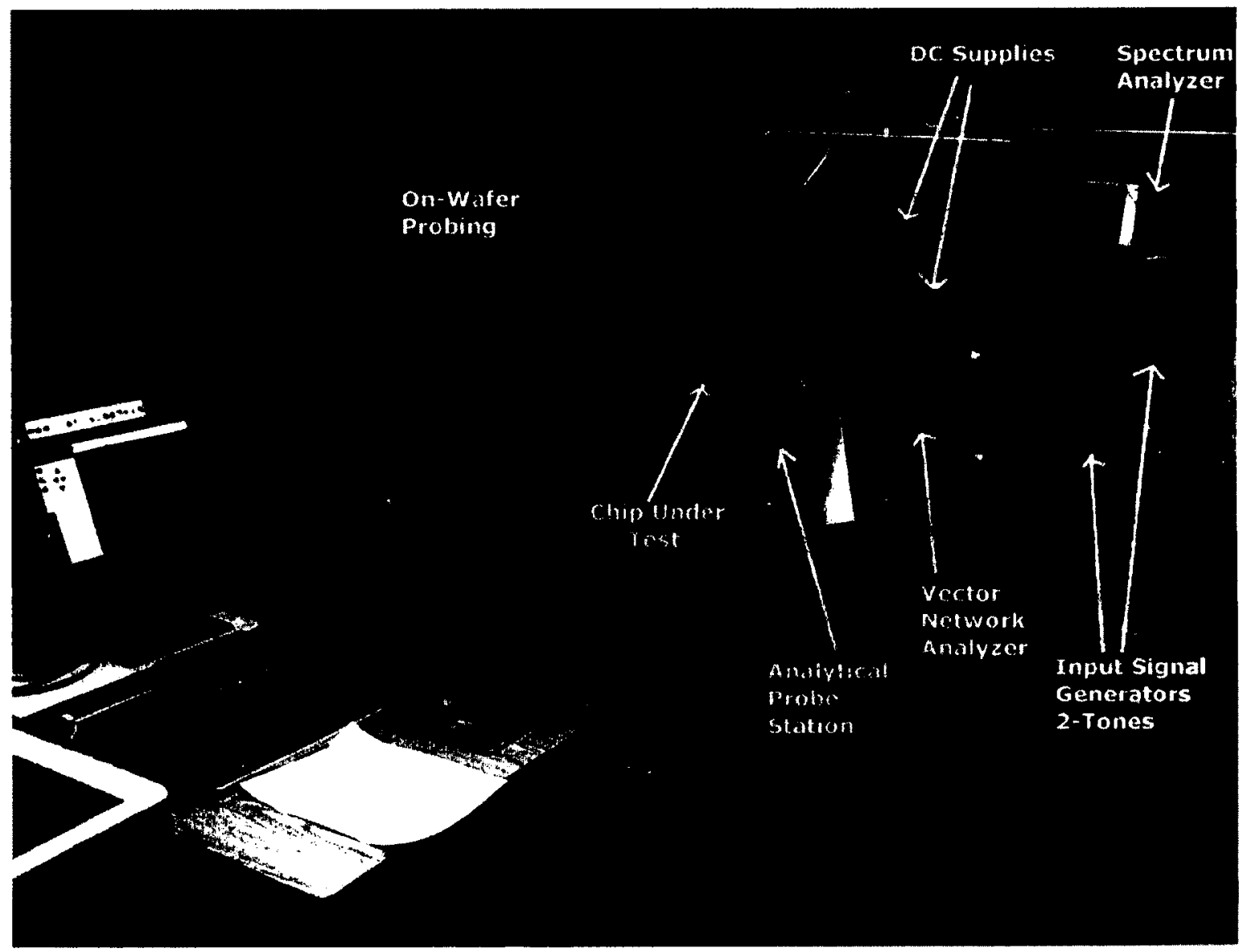

Figure 7.18: Measurement setup of of the $0.13 \mu \mathrm{m}$ RF CMOS three-stage fully-differential linearized CMOS distributed bidirectional amplifier on analytical probe station.

paths with a $10 \mathrm{~dB}$ IIP3 improvement. It is implemented in $0.13 \mu \mathrm{m}$ RF CMOS technology and with a silicon chip area of $1.5 \mathrm{~mm}^{2}$ for use in highly-linear low cost ultra-wideband communications. 


\section{Chapter 8}

\section{Summary of Thesis}

\subsection{Summary}

The emphasis on higher data-rates has driven the industry towards linear modulation techniques such as QPSK, QAM and multi-carrier configurations. Spectral efficiency has become a significant factor in the use of such linear modulation techniques. The result is a signal with a fluctuating envelope which generates intermodulation distortion from the power amplifiers. Linear modulation techniques are more spectral efficient however requires a linear power amplifier. Broadband power amplifiers are important RF components in a wireless communications system. All power amplifiers exhibit inherent nonlinearity which causes spectral regrowth in systems using non-constant envelope digital modulation schemes. The main source of spectral regrowth is the intermodulation distortion of the modulated carrier by nonlinearities in the transmitter power amplifier. Requirements for suppression of spectral regrowth have become more stringent and the improvement in spectral regrowth suppression is the primary reason for using power amplifier linearization techniques.

The main contributions of this thesis research is the realization a fully-integrated highfrequency active broadband linearizer for large IM3 distortion cancellation and spectral regrowth reduction in standard CMOS technology.

In this thesis, we demonstrated a fully-integrated fully-differential linearized CMOS distributed bidirectional amplifier that achieves large $20 \mathrm{~dB}$ IMD3 distortion reduction over ultra-wideband frequency range for both RF paths with least power consumption and 
minimum silicon chip area solution compared to other published linearized DAs. The proposed fully-integrated DA linearization technique greatly suppresses the third-order intermodulation (IM3) distortion with drain and gate transmission-lines stagger-compensated. Reducing and filtering out the DA IM3 distortion by mismatching the gate and drain LC delay-line ladder's time-delay. The proposed fully-differential linearized DA employs a CMOS cross-coupled compensator to enhance the linearity of the DA gain cell with a nonlinear drain capacitance compensator for wider linearization bandwidth. The proposed linearized CMOS bidirectional DA achieves a measured IM3 reduction of $20 \mathrm{~dB}$ in both RF directions with a two-way gain of $5 \mathrm{~dB}$ over ultra-wideband $0.1 \mathrm{GHz}$ to $9.5 \mathrm{GHz}$ frequency of operation eliminating the need of RF switches which degrade performance and increase insertion loss. The proposed linearized DA is fully-differential suppressing substrate noise thus providing better dynamic range compared to single-ended linearized DA designs and with least power consumption of $128 \mathrm{~mW}$ and chip silicon area compared to previous published work. An IIP3 of $18.5 \mathrm{dBm}$ is achieved for both RF paths with a $10 \mathrm{~dB}$ IIP3 improvement. It is implemented in $0.13 \mu \mathrm{m} \mathrm{RF} \mathrm{CMOS} \mathrm{technology} \mathrm{with} \mathrm{a} \mathrm{silicon} \mathrm{chip} \mathrm{area}$ of $1.5 \mathrm{~mm}^{2}$ for use in highly-linear low cost bidirectional ultra-wideband communications. Comparing the proposed CMOS linearized DA in this work [30] to other published ones, the proposed linearized CMOS DA offers a $20 \mathrm{~dB}$ IM3 distortion reduction with $9.5 \mathrm{GHz}$ operational bandwidth and with the least power consumption [30].

The thesis objectives were introduced in Chapter 1. In Chapter 2, modulation schemes effect on RF power amplifier nonlinearity and RFPA linearization techniques were presented. In Chapter 3, distributed amplification principles and transconductor nonlinearity compensation were presented. Various applications of linearized distributed circuit functions were presented in Chapter 4 . Chapter 5 described in details the proposed fullyintegrated linearized CMOS bidirectional distributed amplifier and the proposed highlylinear CMOS cross-coupled compensator transconductor with enhanced tunability. Chapter 6 presented the proposed linearized CMOS bidirectional distributed amplifier layout techniques and considerations. Chapter 7 presented the proposed linearized CMOS bidirectional distributed amplifier experimental test setups and measured results. Chapter 8 drew conclusions of the thesis work and listed thesis contributions. 


\subsection{Future Work}

There are a few interesting new ideas that await exploration in future research of fullyintegrated adaptive RF power amplifier linearizer modules for multi-carrier modulation schemes such as OFDM. Nonlinear signal distortions are generated since most transmitters operate their power amplifiers near saturation to achieve maximum power efficiency. This nonlinear distortion generates spurious spectral sidelobes and spreads the transmitted signal into the adjacent channel causing interference. To resolve this issue, advanced power amplifier linearization modules employing filters will be implemented in the power amplifier optimized transconductor linearization to suppress the sideband lobes.

Future research work will focus on the development of new generation of fully-integrated CMOS linearized broadband power amplifier modules. These advanced linearized power amplifier modules will include build-in optimized transconductors with digitally enhanced distortion compensation system-on-chip. Signal conditioning adaptive linearization circuits will be investigated that allow broadband power amplifiers to run closer to compression (saturation) with improved efficiency and reduced spectral regrowth for linear modulation techniques. However, doing signal processing in DSP takes up more hardware area compared to the proposed solution in this thesis which offers a fully-integrated in standard CMOS technology.

The work presented in this dissertation presents various integrated circuit techniques applied to power amplifiers for spectral regrowth suppression and distortion cancellation. There are a number of improvements that can be made to the proposed linearized bidirectional distributed amplifier such as adaptive digital predistortion circuitry. The added circuitry will assist in maintaining a dynamically updated model of the broadband power amplifier optimizing distortion cancellation. Perform improvements to increase the circuit bandwidth by developing optimized transconductors with reduced gate and drain parasitic capacitance. 


\section{Appendix A}

\section{List of Book, Journal and Conference Publications}

\section{A.1 List of Book, Journal and Conference Publica- tions}

Z. El-Khatib, L. MacEachern and S. A. Mahmoud, "Distributed CMOS Bidirectional Amplifiers: Broadbanding and Linearization Techniques", Springer Analog Signal Processing book series, 2012.

Z. El-Khatib, L. MacEachern and S. A. Mahmoud, "Linearised bidirectional distributed amplifier with $20 \mathrm{~dB}$ IM3 distortion reduction", Journal of Electronics Letters, vol. 46, Jul 2010.

Z. El-Khatib, L. MacEachern and S. A. Mahmoud, "Highly-Linear CMOS Cross-Coupled Compensator Transconductor with Enhanced Tunability", Journal of Electronics Letters, vol. 46 , Jul 2010 .

Z. El-Khatib, L. MacEachern and S. A. Mahmoud, "A Highly-Linearized CMOS Distributed Bidirectional Amplifier with 20 dB IM3 Distortion Reduction for Bidirectional UltraWideband RF-Over-Fiber Communications", In Review stage with IEEE Transactions on Microwave Theory and Techniques Journal, 2011.

Z. El-Khatib, L. MacEachern and S. A. Mahmoud, "CMOS Interleaved Distributed $2 \times 3$ Matrix Amplifier Employing Active Post Distortion and Optimum Gate Bias Linearization Technique", IEEE Canadian Conference on Electrical and Computer Engineering 2010, (CCECE 2010). 
Z. El-Khatib, L. MacEachern and S. A. Mahmoud, "CMOS Distributed Paraphase Amplifier Employing Derivative Superposition Linearization for Wireless Communications", IEEE 2009 Midwest Symposium on Circuits and Systems, 2009, (MWSCAS 2009).

Z. El-Khatib, L. MacEachern and S. A. Mahmoud, "Fully-integrated Multi-band Tunable Linearized CMOS Active Analog Phase Shifter with Active Loss Compensation for Wireless Home Network Multiple Antenna Transceiver Applications", IEEE Proceedings of the 2009 International Symposium on Circuits and Systems, 2009, (ISCAS 2009).

Z. El-Khatib, L. MacEachern and S. A. Mahmoud, "CMOS Distributed Active Power Splitter with Multiple-Gated Transistor Linearization for Ultra-Wideband Applications", IEEE Microsystems and Nanoelectronics Research Conference (MNRC 2009).

Z. El-Khatib, L. MacEachern and S. A. Mahmoud, "A Fully-Integrated Linearized CMOS Distributed Amplifier Based On Multi-Tanh Principle For Radio Over Fiber And UltraWideband Applications", IEEE Radio and Wireless Symposium (RWS 2009).

Z. El-Khatib, L. MacEachern and S. A. Mahmoud, "LTCC-based Ultra-wideband Linearly Tapered Slot Antenna Design Guidelines", IEEE Proceedings of the European Radar Conference 2009, (EuRAD 2009).

Z. El-Khatib, L. MacEachern and S. A. Mahmoud, "Improvement of Carrier Power to Third-Order Intermodulation Distortion Power Ratio in CMOS Distributed Amplifiers", IEEE 20th International Conference on Microelectronics (ICM) 2008 - Circuits and Systems.

Z. El-Khatib, L. MacEachern and S. A. Mahmoud, "Fully-Integrated CMOS Distributed Amplifier as Tunable UWB Active Duplexer for Short Range Wireless Communication", IEEE Microsystems and Nanoelectronics Research Conference (MNRC 2008).

Z. El-Khatib, L. MacEachern and S. A. Mahmoud, "A Fully-Integrated Linearized CMOS Bidirectional Distributed Amplifier as UWB Active Circulator", IEEE 20th International Conference on Microelectronics (ICM) 2008 - Circuits and Systems. 


\section{Bibliography}

[1] Hittite Microwave, "High IP3 Mixers for Cellular Applications," Hittite Product Application Notes, 2010.

[2] L. Larson and P. Asbeck, "Advanced digital linearization approaches for wireless RF power amplifiers," IEEE Circuits and Systems Workshop: System-on-Chip - Design, Applications, Integration and Software, pp. 1-7, 2008.

[3] R. Sadhwani et al, "Adaptive CMOS Predistortion Linearizer for Fiber-Optic Links," Journal of Lightwave Technology, Nov 2003.

[4] H. S. Black, "Stabilized Feed-Back Amplifiers," Proceedings of The IEEE, JUNE 1984.

[5] S. Ghoniemy, and L. MacEachern, and S. A. Mahmoud, "Performance Analysis and Enhancement of RF/Fiber Optical Interface for Microcellular Wireless Transceivers," Wireless and Optical Communications, July 2003.

[6] T. W. Kim et al, "Highly Linear Receiver Front-End Adopting MOSFET Transconductance Linearization by Multiple Gated Transistors," IEEE Journal of Solid-State Cincuits, Jan 2004.

[7] K. Tsun Mok et al, "Linearised Distributed Amplifier with Low Linearisation Loss," Electronics Letters, pp. 2011-2014, Jan 2004.

[8] D. Smith, et al, " $480 \mathrm{Mbps}$ Ultra-Wideband Radio-over-Fibre Transmission Using a 1310/1550nm Reflective Electro-absorption Transducer and Off-The-Shelf Components," IEEE JOURNAL OF SOLID-STATE CIRCUITS, April 1997.

[9] M.P. Thakur and Y. Ben-Ezra, "480 Mbps, Bi-Directional, Ultra-Wideband Radio-Over-Fiber Transmission Using a 1308/1564 nm Reflective Electro-Absorption Transducer and Commercially Available VCSELs," Journal of Lightwave Technology, Feb 2009.

[10] F. Raab and N. Sokal, "Power Amplifiers and Transmitters for RF and Microwave," IEEE Transactions on Microwave Theory And Techniques, vol. 50, March 2002.

[11] X. N. Fernando and A. B. Sesay, "Higher Order Adaptive Filter Based Predistortion for Nonlinear Distortion Compensation of Radio over Fiber Links," IEEE International Conference on Communications, Feb 2000.

[12] L. Vreede and M. van der Heijden, IEEE BCTM.

[13] P. Wambacq and W. Sansen, "Distortion Analysis of Analog Integrated Circuits," Kluwer Acadamic Publishers, October 1998.

[14] S. Maas, "Nonlinear Microwave Circuits," Artech House, 1988.

[15] M. Mbabele and C.S. Aitchison, "Third Order Intermodulation Improvement in Distributed Amplifiers," IEEE European Microwave Conference, pp. 1-4, Oct 2001. 
[16] R. A. Minasian, "Intermodulation Distortion Analysis of MESFET Amplifiers Using the Volterra Series Representation," IEEE Transactions On Microwave Theory And Techniques, vol. 28, Jan 1980.

[17] D.K. Paul and G. Parkinson, Microwave and Optical Technology Letters.

[18] C. H. Lee et al, "Enhanced Performance of ROF Link For Cellular Mobile Systems Using Postdistortion Compensation," Personal, Indoor and Mobile Radio Communications, Sept 2004.

[19] O. P. Leisten et al., "Distributed Amplifiers as Duplexer/Low Crosstalk Bidirectional Elements in S-Band," Electronics Letters, vol. 24, pp. 188-189, Mar 1988.

[20] J. W. Byrne and J. B. Beyer, "A Highly Directive, Broadband, Bidirectional Distributed Amplifier," IEEE International Microwave Symposium Digest, vol. 1, pp. 188-189, June 1989.

[21] B. Sundaram and P. N. Prasad, "A Novel Electronically Tunable Active Duplexer for Wireless Transceiver Applications," IEEE Transactions on Microwave Theory and Techniques, vol. 54, June 2006.

[22] M. J. Cryan et al, "Analysis and Design of Integrated Active Circulator Antennas," IEEE Transactions On Microwave Theory And Techniques, vol. 48, no. 6, June 2000.

[23] K. Lau and C. Chan, "Self-Biased Distributed Amplifier: Linearity Improvement and Efficiency Enhancement," Microwave and Optical Technology Letters, Oct 2008.

[24] K. Tsun Mok et al, "Control of Circuit Distortion by the Derivative superposition Method," IEE Colloquium Wideband Circuits, Modelling and Technique, pp. 2011-2014, Feb 1996.

[25] M. Iwamoto and C.P. Hutchinson, "Optimum Bias Conditions for Linear Broadband InGaP/GaAs HBT Power Amplifiers," IEEE International Microwave Symposium Digest, vol. 2, pp. 901-904, June 2002.

[26] B. Gilbert, "The Multi-tanh Principle: A Tutorial Overview," IEEE Journal of Solid-State Circuits, vol. 33, pp. 188-189, Jan 1998.

[27] C. Lu and A. H. Pham and D. Livezey, "On the Feasibility of CMOS Multiband Phase Shifters for Multiple-Antenna Transmitters," IEEE Microwave and Wireless Components Letters, vol. 3, p. 2100, May 2006.

[28] Z. El-Khatib and L. MacEachern and S. A. Mahmoud, "A Fully-Integrated Linearized CMOS Distributed Amplifier Based On Multi-Tanh Principle For Radio Over Fiber and Ultra-Wideband Applications," IEEE Radio and Wireless Symposium, vol. 33, pp. 188-189, Jan 2009.

[29] G. Shiroma and R. Y. Miyamoto and W. A. Shiroma, "A Combined Distributed Amplifier/TrueTime-Delay Phase Shifter for Broadband Self-Steering Arrays," IEEE MTT-S International Microwave Symposium Digest, vol. 16, p. 4, June 2005.

[30] Z. El-Khatib and L. MacEachern and S. A. Mahmoud, "Linearised Bidirectional Distributed Amplifier with 20 dB IM3 Distortion Reduction," Electronics Letters, Jul 2010.

[31] C. Lu and A. H. Pham, "Linearization of CMOS Broadband Power Amplifiers Through Combined Multigated Transistors and Capacitance Compensation," IEEE International Symposium on Circuits and Systems, vol. 55, Nov 2007.

[32] H. P. E. Stern and S. A. Mahmoud and L. E. Stern, "Communication Systems: Analysis and Design," Pearson Prentice Hall, 2004.

[33] J. Rogers and C. Plett, Radio Frequency Integrated Circuit Design. Artech House Inc, 2010.

[34] B. Razavi, RF Microelectronics. Englewood Cliffs, NJ: Prentice Hall, 1998. 
[35] R. Lyons, "Quadrature Signals: Complex, But Not Complicated," DSP Training, 2000.

[36] T. H. Lee, "The Design of CMOS Radio Frequency Integrated Circuits," Cambridge University Press, pp. 423-425, March 2004.

[37] D. R. Webster and A. Parker, "Derivative Superposition - A Linearization Technique for Ultra Broadband Systems," IEEE Colloquium Wideband Circuits, Modelling and Techniques, June 1996.

[38] J. Vuolevi and T. Rahkonen, "Distortion in RF Power Amplifiers," Artech House, 2003.

[39] G. Gonzalez, "Microwave Transistor Amplifiers: Analysis and Design," Prentice Hall, 1997.

[40] M. Steer and Lawrence E. Larson, "The Impact of RF Front-End Characteristics on the Spectral Regrowth of Communications Signals," IEEE Transactions on Microwave Theory And Techniques, Jun 2005.

[41] G. Zhou, "Analysis of Spectral Regrowth of Weakly Nonlinear Power Amplifiers," IEEE International Conference on Acoustics, Speech, and Signal Processing, Jun 2000.

[42] I. J. Bahl, "Fundamentals of RF and Microwave Transistor Amplifiers," Wiley, pp. 40-43, 2009.

[43] Q. Wu and R. Larkin, "Linear RF Power Amplifier Design for CDMA Signals," IEEE MTT-S International Microwave Symposium Digest, Jun 1996.

[44] J. Sevic and J. Staudinger, "Simulation of Adjacent-Channel Power for Digital Wireless Communication Systems," IEEE MTT-S International Microwave Symposium Digest, Jun 1996.

[45] N. B. Carvalho and J. C. Pedro, "Compact Formulas To Relate ACPR AND NPR TO TWO-TONE IMR and IP3," Microwave Joumal, Dec 1999.

[46] W. Struble, et al, "Understanding Linearity in Wireless Communication Amplifiers," IEEE JOURNAL OF SOLID-STATE CIRCUITS, April 1997.

[47] N. B. Carvalho and J. C. Pedro, "Intermodulation Distortion in Microwave and Wireless Circuits," Artect House, 2003.

[48] V. Petrovic and W. Gosling, "Polar-Loop Transmitter," Electronics Letters, pp. 286-287, May 1979.

[49] M. A. Briffa and M. Faulkner, "Stability Analysis of Cartesian Feedback Linearisation for Amplifiers with Weak Nonlinearities," IEE Proceedings on Communications, pp. 212 218, 1996.

[50] P. Kenington, "High-Linearity RF Amplifier Design," Artech House, 2000.

[51] Hittite Microwave, "Linearisation of RF Multicarrier Amplifiers using Cartesian Feedback," Electronics Letters, vol. 45, pp. 1110-1111, 1994.

[52] V. Petrovic and W. Gosling, "Polar-loop Transmitter," Electronics Letters, pp. 286-288, 1979.

[53] S. Cripps, "Advanced Techniques in RF Power Amplifier Design," Artech House, 2002.

[54] J. K. Cavers, "Amplifier Linearization Using a Digital Predistorter with Fast Adaptation and Low Memory Requirements," IEEE Transactions on Vehicular Technology, pp. 374-382, 1990.

[55] M. K. Nezami, "Fundamentals of Power Amplifier Linearization Using Digital Pre-Distortion," High Frequency Design Electronics, 2004.

[56] E.L. Ginzton and W. R. Hewlett and J. H. Jasberg and J. D. Noe, "Distributed Amplification," Proc. IRE, vol. 36, pp. 956-969, August 1948.

[57] Thomas T. Y. Wong, Fundamentals of Distributed Amplification. Artech House Inc, 1993.

[58] Heutmaker and S. Michael, "Error Vector and Power Amplifer Distortion," Proceedings of the 1997 Annual Wireless Communications, Aug 2003. 
[59] B. Razavi, Design of integrated circuits for optical communications. New York: McGraw-Hill, 2003.

[60] - Design of analog CMOS integrated circuits. New York: McGraw-Hill, 2001.

[61] J. B. Beyer et al, "MESFET Distributed Amplifier Design Guidelines," IEEE Transactions On Microwave Theory And Techniques, vol. 32, no. 3, Mar 1984.

[62] J. B. Prasad et al, "Power-Bandwidth Considerations in the Design of MESFET Distributed Amplifiers," IEEE Transactions On Microwave Theory And Techniques, vol. 36, no. 7, Jul 1988.

[63] Y. Ayasli et al, "A Monolithic GaAs 113-GHz Traveling-Wave Amplifier," IEEE Transactions On Microwave Theory And Techniques, vol. 30, no. 7, May 1982.

[64] H. Ahn and D. Allstot, "A $0.5-8.5 \mathrm{GHz}$ fully differential CMOS distributed amplifier," IEEE Microwave Theory and Techniques, vol. 43, p. 6, August 2002.

[65] C. Lu and A. H. Pham and D. Livezey, "On the Linearity of CMOS Multi-band Phase Shifters," IEEE Silicon Monolithic Integrated Circuits in RF Systems Digest, vol. 1, p. 4, Jan 2006.

[66] C. A. T. Salama, "A Novel C-Band CMOS Phase Shifter for Communication Systems," IEEE International Symposium on Circuits and Systems, vol. 2, p. 316, May 2003.

[67] C. Lu and A. H. Pham and D. Livezey, "A Novel Multiband Phase Shifter with Loss Compensation in $180 \mathrm{~nm}$ RF CMOS Technology," IEEE Midwest Symposium on Circuits and Systems, vol. 1, p. 806, Aug 2005.

[68] D.M. Pozar, Microwave Engineering. Addison Wesley Publishing Company, 1990.

[69] S. Deibele et al, "Attenuation Compensation in Distributed Amplifier Design," IEEE Transactions on Microwave Theory and Techniques, vol. 37, no. 9, p. 14251433, Sep. 1989.

[70] D.G. Sarma, "On Distributed Amplification," Proc. Inst. Elect. Eng., vol. 102B, p. 689697, 1954.

[71] P. Popplewell and V. Karam and A. Shamim et al., "A 5.2-GHz BFSK Transceiver Using InjectionLocking and an On-Chip Antenna," IEEE Journal of Solid-State Cincuits, vol. 43, p. 6, April 2008.

[72] P.B. Kenington and R. J. Wilkinson and J. D. Marvill, "Broadband linear amplifier design for a PCN base-station," IEEE Vehicular Technology Conference, pp. 155-160, May 1991.

[73] K. Kimura, "The Ultra-Multi-Tanh Technique for Bipolar Linear Transconductance Amplifiers," IEEE Transactions on Circuits and Systems, vol. 44, no. 4, Apr 1997.

[74] Z. M. Li and P. N. Prasad, "Optimal design of low crosstalk, wideband, bidirectional distributed amplifiers," IEEE International Microwave Symposium Digest, June 1996.

[75] W.S. Percival, "Thermionic valve circuits," January 1936.

[76] C. Lu and A. H. Pham and D. Livezey, "Development of Multiband Phase Shifters in 180-nm RF CMOS Technology with Active Loss Compensation," IEEE Transactions on Microwave Theory and Techniques, vol. 54, Jan 2006.

[77] A. Safarian and P. Heydari, "CMOS Distributed Active Power Combiners and Splitters for MultiAntenna UWB Beamforming Transceivers," IEEE Journal of Solid-State Cincuits, July 2007.

[78] G. M. Hilder, "A hybrid 2 - $40 \mathrm{GHz}$ High Power Distributed Amplifier using an Active Splitter and Combined Drain Line Configuration," IEE Colloq. Millimetre Wave Transistors and Circuits, March 1991.

[79] Z. El-Khatib and L. MacEachern and S. A. Mahmoud, "CMOS Distributed Paraphase Amplifier Employing Derivative Superposition Linearization for Wireless Communications," IEEE Midwest Symposium on Circuits and Systems, May 2009. 
[80] — "CMOS Distributed Active Power Splitter with Multiple-Gated Transistor Linearization for Ultra-Wideband Applications," IEEE Microsystems and Nanoelectronics Research Conference, Oct 2009.

[81] H. M. Pan and L. E. Larson, "Highly Linear Bipolar Transconductor For Broadband High-Frequency Applications with Improved Input Voltage Swing," IEEE Transactions on Microwave Theory and Techniques, vol. 49, June 2007.

[82] B. Kim and J. Ko and K. Lee, "A New Linearization Technique for MOSFET RF Amplifier Using Multiple-Gated Transistor," IEE Microwave and Guided Wave Letters, Sept 2000.

[83] S. Tanaka and A. A. Abidi, "A Linearization Technique for CMOS RF Power Amplifiers," IEEE Symposium VLSI Circuits Digest Technical Papers, Sept 1997.

[84] S. Kang and B. Kim, "Linearity Analysis of CMOS for RF Applications," IEEE Transactions on Microwave Theory and Techniques, March 2003.

[85] Z. El-Khatib and L. MacEachern and S.A. Mahmoud, "A 0.1-12 GHz Fully Differential CMOS Distributed Amplifer Employing a Feedforward Distortion Cancellation Technique," IEEE International Symposium on Circuits and Systems, vol. 1, May 2004.

[86] P. Popplewell and V. Karam and A. Shamim et al., "A 5.2-GHz BFSK Transceiver Using InjectionLocking and an On-Chip Antenna," IEEE Journal of Solid-State Circuits, vol. 43, April 2008.

[87] A. Shamim, "Silicon Differential Antenna/Inductor for Short Range Wireless Communication Applications," Canadian Conference on Electrical and Computer Engineering, May 2006.

[88] T. Chen and J. Chien and and L. Lu, "A 45.6-GHz Matrix Distributed Amplifier in 0.18um CMOS," IEEE Custom Integrated Circuits Conference, June 2005.

[89] S. Chu and Y. Tajima, "IEEE MTT-S Digest," A Novel 4-18 GHz Monolithic Matrix Distributed Amplifier, May 1989.

[90] K. Niclas, "Recent Advances in the Design of Matrix Amplifiers," Archive fur Elektrotechnik, May 1991.

[91] Y. Chen and J. B. Beyer and V. Sokolov and J. Culp, "Design and Performance of 20 dB Gain Two-tier Matrix Distributed Amplifer," Electronics Letters, Mar 1991.

[92] K. Kobayashi and R. Esfandiari and D. Streit, "GaAs HBT Wideband Matrix Distributed and Darlington Feedback Amplifier to $24 \mathrm{GHz}$," IEEE Transactions on Microwave Theory and Techniques, Jan 1993.

[93] J. Park and D. Allstot, "A 12.5GHz RF Matrix Amplifier in 180nm SOI CMOS," IEEE International Symposium on Circuits and Systems, May 2004.

[94] B. Gilbert, "The Multi-tanh Principle: A tutorial Overview," IEEE Joumal of Solid-State Circuits, vol. 33, no. 1 , January 1998.

[95] V. Arapin and L. E. Larson, "Linearization of CMOS LNAs Via Optimum Gate Biasing," IEEE International Symposium on Cincuits and Systems, May 2004.

[96] Y. W. Chen and J. B. Beyer and S. N. Prasad, "MESFET Wideband Distributed Paraphase Amplifier," International Journal of Electronics, Aug 1985.

[97] Y. Chen and J. B. Beyer and V. Sokolov and J. Culp, "A 11GHz Hybrid Paraphase Amplifier," IEEE International Solid-State Circuits Conference, Feb 1986.

[98] M. Levent-Villegas, "X-Band Paraphase Amplifier," Electronics Letters, May 1984. 
[99] Z. El-Khatib and L. MacEachern and S. A. Mahmoud, "Fully-integrated Multi-band Tunable Linearized CMOS Active Analog Phase Shifter with Active Loss Compensation for Wireless Home Network Multiple Antenna Transceiver Applications," IEEE Proceedings of the 2009 International Symposium on Circuits and Systems, May 2009.

[100] —, "Highly-Linear CMOS Cross-Coupled Compensator Transconductor with Enhanced Tunability," Electronics Letters, Nov 2010.

[101] D. Webster et al, "Control of circuit distortion by the derivative superposition method," IEEE Microwave Guided Wave Letters, Mar 1996.

[102] B. Toole et al, "RF Circuit Implications of Moderate Inversion Enhanced Linear Region in MOSFETs," IEEE Transactions On Circuits And Systems, Feb 2004.

[103] M. Mbabele and C.S. Aitchison, "A Cascode Amplifier Nonlinearity Correction Technique," International Solid-State Circuits Conference, pp. 188-189, Feb 1981.

[104] — "Intermodulation Distortion Analysis of MESFET Amplifiers Using the Volterra Series Representation," IEEE Transactions On Microwave Theory And Techniques, Jan 1980.

[105] —-, "Third Order Intermodulation Improvement in Distributed Amplifiers," IEEE European Microwave Conference, Oct 2001.

[106] J. Kenney and L. Stevenson, "Power Amplifier Spectral Regrowth for Digital Cellular and PCS Applications," Microwave Journal, pp. 851-854, Oct 1995.

[107] P. A. Quinn et al, "A Cascode Amplifier Nonlinearity Correction Technique," IEEE Intermational Solid-State Circuits Conference, Feb 1981.

[108] D. J. Allstot, "A Full-range All-Pass Variable Phase Shifter for Multiple Antenna Receivers," IEEE Transactions on Microwave Theory and Techniques, vol. 16, May 2005.

[109] A. Zolfaghari and A. Chen and B. Razavi, "Stacked Inductors and Transformers in CMOS technology," IEEE Journal of Solid-State Circuits, vol. 36, no. 4, April 2001.

[110] T. Dickson and S. Voinigescu et al, "30-100-GHz inductors and Transformers for Millimeter-Wave (Bi)CMOS Integrated Circuits," IEEE Transaction on Microwave Theory and Techniques, vol. 53, no. 1, 2005.

[111] C. P. Yue et al, "Design strategy of on-chip inductors for highly integrated RF systems," Design Automation Conference, pp. 982-987, Jun 1999.

[112] Y. Koutsoyannopoulos et al, "Performance Limits Of Planar And Multi-Layer Integrated Inductors," IEEE International Symposium on Circuits and Systems, May 2000.

[113] J.N. Burghartz et al, "Progress in RF inductors on Silicon - Understanding Substrate Losses," Tech. Digest IEDM, p. $523526,1998$.

[114] J. Gil et al, "A Simple Wide-Band On-Chip Inductor Model for Silicon-Based RF ICs," IEEE Transactions On Microwave Theory And Techniques, vol. 51, no. 9, Sept 2003.

[115] J. Long and M. A. Copland, "The modeling, characterization, and design of monolithic inductors for silicon RF IC's," IEEE Journal Solid-State Circuits, vol. 32, pp. 357-369, March 1997.

[116] C.P. Yue and S.S. Wong, "On-chip Spiral Inductors with Patterned Ground Shields for Si-based RF ICs," IEEE Journal of Solid-State Circuits, vol. 33, pp. 743-752, May 1998.

[117] B. Razavi, "Prospects of CMOS technology for high-speed optical communication circuits," IEEE Journal of Solid-State Circuits, vol. 37, no. 9, pp. 1135-1145, Sep 2002. 
[118] IBM Foundry and Manufacturing Services Education, "IBM CMOS8RF Process," IBM CMOS8RF Technology Design Kit Manual http://www.ibm.com/chips, 2007.

[119] H. Cho et al, "A Three-Step Method for the De-Embedding of High Frequency S-Parameter Measurements," IEEE Transactions on Electron Devices, vol. 38, no. 6, p. 13711375, Jun.

[120] A. M. Niknejad, "Modeling of Passive Elements with ASITIC," IEEE Radio Frequency Integrated Circuits Symposium, 2002.

[121] W. B. Kuhn et al, "Spiral inductor substrate loss modeling in silicon RF ICs," IEEE Radio and Wireless Conference, pp. 305-308, Aug 1998.

[122] M. Koolen, Microelectronic Engineering: On-wafer High-frequency Device Characterization. Elsevier Science Publisher, 1992.

[123] Agilent Technologies, "Agilent E4438C ESG Vector Signal Generator Data Sheet," www.agilent.com/find/esg.

[124] D.R. Webster and A.E. Parker, "Derivative Superposition - A Linearisation Technique for Ultra Broadband Systems," IEE Colloquium Wideband Circuits, Modelling and Techniques, pp. 901-904, June 1996. 